\title{
Validation of \\ Nuclear Criticality Safety Software and \\ 27 Energy Group ENDF/B-IV Cross Sections
}

August 1994

By

B. L. Lee, Jr.

Martin Marietta Utility Services, Inc PORTSMOUTH GASEOUS DIFFUSION PLANT

P.O. Box 628 Piketon, Ohio 45661

Under Contract USECHQ-93-C-0001

to the

U.S. ENRICHMENT CORPORATION 


\section{NOTICE}

This report was prepared as an account of work sponsored by an agency of the United States Government. Neither the United States Government nor any agency thereof, nor any of their employees makes any warranty, express or implied, or assumes any legal liability or responsibility for the accuracy, completeness, or usefulness of any information, apparatus, product, or process disclosed, or represents that its use would not infringe privately owned rights. Reference herein to any specific commercial product, process, or service by trade name, trademark, manufacturer, or otherwise does not necessarily constitute or imply its endorsement, recommendation, or favoring by the United States Government or any agency thereof. The views and opinions of authors expressed herein do not necessarily state or reflect those of the United States Government or any agency thereof.

Available to DOE and DOE Contractors from the Office of Scientific and Technical Information, P. O. Box 62, Oak Ridge, TN 37831; prices available from (615) 576-8401, FTS/626-8401.

Available to the public from the National Technical Information Service, U. S. Department of Commerce, 5285 Port Royal Road, Springfield, VA 22161. 


\section{DISCLAIMER}

Portions of this document may be illegible in electronic image products. Images are produced from the best available original document. 


\section{DISTRIBUTION}

\section{MARTIN MARIETTA UTILITY SERVICES, INC.}

\section{PORTSMOUTH}

R. Newvahner

J. Harris

C. Haught

R. Oxenham

R. Lemming
Technical Review (3)

D. D'Aquila (10)

Central Files (2)

X-710 Technical Library (2)

C. Dean
R. Tayloe
B. Lee (4)

\section{OAK RIDGE}

W. Jordan 


\begin{abstract}
The validation documented in this report is based on calculations that were executed during June through August 1992, and was completed in June 1993. The statistical analyses in Appendix C and Appendix D were completed in October 1993.

This validation gives Portsmouth NCS personnel a basis for performing computerized KENO V.a calculations using the Martin Marietta Nuclear Criticality Safety Software. The first portion of the document outlines basic information in regard to validation of NCSS using ENDF/B-IV 27-group cross sections on the IBM3090 at ORNL. A basic discussion of the NCSS system is provided, some discussion on the validation database and validation in general. Then follows a detail description of the statistical analysis which was applied. The results of this validation indicate that the NCSS software may be used with confidence for criticality calculations at the Portsmouth Gaseous Diffusion Plant. When the validation results are treated as a single group, there is $95 \%$ confidence that $99.9 \%$ of future calculations of similar critical systems will have a calculated $\mathrm{K}_{\mathrm{eff}}>0.9616$. Based on this result the Portsmouth Nuclear Criticality Safety Department has adopted the calculational acceptance criteria that a $k_{\text {eff }}+2 \sigma$ $\leq 0.95$ is safety subcritical. The validation of NCSS on the IBM 3090 at ORNL was extended to include NCSS on the IBM 3090 at K-25.
\end{abstract}


INTRODUCTION $\ldots \ldots \ldots \ldots \ldots \ldots \ldots \ldots \ldots \ldots \ldots \ldots \ldots \ldots \ldots \ldots \ldots$

CALCULATIONAL PROCEDURE $\ldots \ldots \ldots \ldots \ldots \ldots \ldots \ldots \ldots \ldots \ldots \ldots \ldots$

VALIDATION DATABASE $\ldots \ldots \ldots \ldots \ldots \ldots \ldots \ldots \ldots \ldots \ldots \ldots \ldots$

STATISTICAL ANALYSIS $\ldots \ldots \ldots \ldots \ldots \ldots \ldots \ldots \ldots \ldots \ldots \ldots$

EXTENSION TO THE K-25 IBM3090 $\ldots \ldots \ldots \ldots \ldots \ldots \ldots \ldots \ldots \ldots$

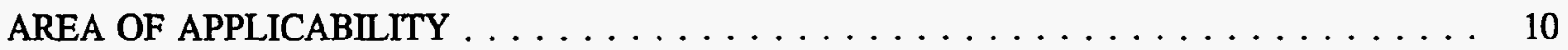

RECOMMENDATIONS FOR THE FUTURE $\ldots \ldots \ldots \ldots \ldots \ldots \ldots \ldots \ldots \ldots \ldots$

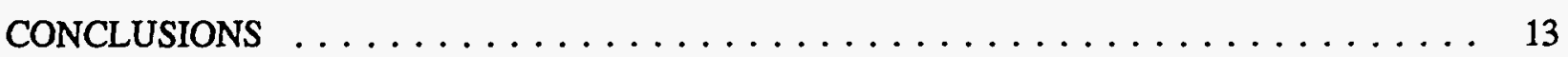

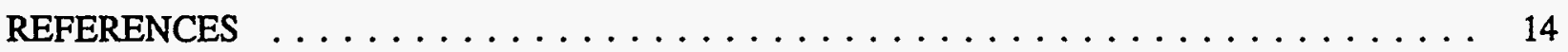

APPENDIX A CALCULATION OF $\mathrm{K}^{*} \ldots \ldots \ldots \ldots \ldots \ldots \ldots \ldots \ldots \ldots \ldots \ldots \ldots \ldots$

APPENDIX B1 TABLE 1 RESULTS $\ldots \ldots \ldots \ldots \ldots \ldots \ldots \ldots \ldots \ldots \ldots \ldots \ldots \ldots$ B-1

APPENDIX B2 TABLE 2 RESULTS $\ldots \ldots \ldots \ldots \ldots \ldots \ldots \ldots \ldots \ldots \ldots$ B-5

APPENDIX B3 TABLE 3 RESULTS $\ldots \ldots \ldots \ldots \ldots \ldots \ldots \ldots \ldots \ldots$ B-7

APPENDIX B4 TABLE 4 RESULTS $\ldots \ldots \ldots \ldots \ldots \ldots \ldots \ldots \ldots \ldots$ B-14

APPENDIX B5 TABLE 5 RESULTS $\ldots \ldots \ldots \ldots \ldots \ldots \ldots \ldots \ldots \ldots$ B-18

APPENDIX B6 TABLE 6 RESULTS $\ldots \ldots \ldots \ldots \ldots \ldots \ldots \ldots \ldots \ldots$ B-29

APPENDIX C STATISTICAL ANALYSIS BASED ON SEGREGATED GROUPS $\ldots \ldots \ldots$ C-1

APPENDIX D CALCULATED RESULTS AND EXPERIMENTAL PARAMETERS . . . . . D D-1

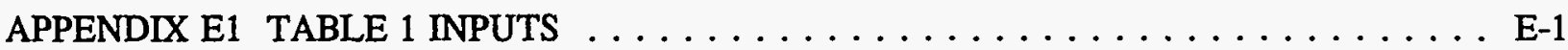

APPENDIX E2 TABLE 2 INPUTS $\ldots \ldots \ldots \ldots \ldots \ldots \ldots \ldots \ldots \ldots \ldots \ldots \ldots \ldots$

APPENDIX E3 TABLE 3 INPUTS $\ldots \ldots \ldots \ldots \ldots \ldots \ldots \ldots \ldots \ldots \ldots \ldots \ldots \ldots$ 
APPENDIX E4 TABLE 4 INPUTS $\ldots \ldots \ldots \ldots \ldots \ldots \ldots \ldots \ldots \ldots \ldots$

APPENDIX E5 TABLE 5 INPUTS $\ldots \ldots \ldots \ldots \ldots \ldots \ldots \ldots \ldots \ldots$

APPENDIX E6 TABLE 6 INPUTS $\ldots \ldots \ldots \ldots \ldots \ldots \ldots \ldots \ldots \ldots \ldots$. . . . . . . . . . 


\section{VALIDATION OF NUCLEAR CRITICALITY SAFETY SOFTWARE AND \\ 27 ENERGY GROUP ENDF/B-IV CROSS SECTIONS}

\section{INTRODUCTION}

The purpose of this validation is to provide Portsmouth Gaseous Diffusion Plant Nuclear Criticality Safety Personnel with confidence that the Martin Marietta Energy Systems Nuclear Criticality Safety Software (NCSS) is valid for use in the analysis and subsequent evaluation of various plantsite activities involving enriched uranium.

This validation was patterned after a previous validation study documented in ORNL/CSD/TM-238 for the Y12CSG implementation of Keno V.a. The Y12CSG validation was considered an appropriate pattern to follow because the Y12CSG software was a forerunner to the current NCSS software and the range of experiments which were previously validated encompass the range of systems which might be encountered at PORTS. The validation was conducted using ENDF/B-IV 27 energy group cross sections with SCALE 4.1 and KENO V.a. All calculations were performed on the IBM-3090 located at the Oak Ridge National Laboratory in Oak Ridge, Tennessee, and consisted of activating 3 functional modules of the SCALE 4.1 package, 000008 (BONAMI), 000002 (NITAWL), and 000009 (KENO V.a). The modules were accessed through the SCALE 4.1 control module CSAS25. The calculational sequence accessed two basic data libraries, a library of standard compositions (STDCOMP), and the 27 energy group master cross section library (NXS027). Specific data to uniquely identify accessed datasets is given in the table below.

\begin{tabular}{|c|c|c||}
\hline $\begin{array}{c}\text { Module } \\
\text { Name }\end{array}$ & $\begin{array}{c}\text { Creation } \\
\text { Date }\end{array}$ & $\begin{array}{c}\text { Dataset } \\
\text { Name }\end{array}$ \\
\hline \hline $\begin{array}{c}\text { o00008 } \\
\text { (BONAMI) }\end{array}$ & 91.205 & NCSS.ZAZ39461.BONAMI.V1R000.S000.LOAD \\
\hline $\begin{array}{c}\text { o00002 } \\
\text { (NITAWL) }\end{array}$ & 91.345 & NCSS.ZAZ39461.NITAWL.V1R000.S000.LOAD \\
\hline $\begin{array}{c}\text { o00009 } \\
\text { KENO V.a) }\end{array}$ & 92.010 & NCSS.ZAZ39461.KENOVA.V1R000.S000.LOAD \\
\hline CSAS25 & 92.014 & NCSS.ZAZ39461.CSAS25.V1R000.S000.LOAD \\
\hline STDCMP & $8 / 19 / 91$ & NCSS.ZAZ39461.STDCMP.V1R000.DATA \\
\hline NXS027 & $8 / 14 / 91$ & NCSS.ZAZ39461.NXS027.V1R000.DATA \\
\hline \hline
\end{tabular}

Approximately 245 cases, consisting of 101 highly enriched ( $>90 \% \mathrm{U}^{235}$ ) and 144 low enriched ( $<5 \%$ $\mathrm{U}^{235}$ ) cases were run. These cases consisted of various $\mathrm{UO}_{2} \mathrm{~F}_{2}$ and $\mathrm{UO}_{2}\left(\mathrm{NO}_{3}\right)_{2}$ solution systems, oxide-water systems, metal-water systems, and several miscellaneous combinations of uranium and moderating materials. All of the executed cases have definite applicability to operations at the Portsmouth plant except the metal systems. Several metal systems were included for completeness to allow the 
established area of applicability for the NCSS software at Portsmouth to be extended to include dry unmoderated systems with a hard neutron spectrum.

The data obtained from the calculations was tabulated and analyzed by personnel in the NCS department at the Portsmouth plant, who in consultation with the statistical analysis section, concluded that the lower limit for $\mathrm{k}_{\text {eff }}$ with $95 \%$ confidence and a $99.9 \%$ one-sided tolerance limit was 0.9616 . For the anticipated range of materials to be encountered at PORTS, the NCSS software is safe for NCS use at PORTS provided $k_{\text {eff }}+2 \sigma$ does not exceed a value of 0.95 . The margin between 0.95 and 0.9616 has been adopted by the NCS department at Portsmouth as an additional margin of safety and to allow consistency with the value of 0.95 that has traditionally been used. 


\section{CALCULATIONAL PROCEDURE}

The CSAS25 calculational sequence allows simplified data input to the accessed functional modules BONAMI, NITAWL, and KENO V.a. It accesses the standard composition library to apply preset mixtures of materials available in the library, can calculate atomic densities for both mixtures and solutions, and in addition will generate the required input for the NITAWL module, allowing various options for treatment of the cross sections in the resonance regions for both homogenous and heterogenous systems to be used.

Following execution of the CSAS25 control module is the functional module BONAMI. The primary purpose of this module, when used with the 27 energy group cross section, as was done in this validation, is to read the master cross section library, select the required cross sections, and then write a smaller master library, unique to the problem under examination, for use by the NITAWL module.

The NITAWL functional module performs resonance region calculations on the selected cross sections for resonance absorbers based on the Nordheim Integral Transform method. Some flexibility is allowed to the analyst in selecting the method to be used for the treatment based on the specific problem. The specific treatment to be used is specified by the keywords INFHOMMEDIUM, LATTICECELL, and MULTIREGION in the problem input. With the infhommedium option, the entire fissile mixture is treated as if it were an infinite homogenous medium, while with the latticecell option, the fissile medium is treated as if it consisted of multiple repeated cells. The multiregion option is similar to the latticecell option, with the exception that the fissile medium is treated as if only a single lattice cell existed, with no repetition.

The major functional module of the CSAS25 sequence is the KENO V.a module. The major function of KENO V.a is to calculate the $k_{\text {eff }}$ of a system. It is a substantial revision of KENO IV, however it differs in that KENO V.a now supports more extensive geometry features such as nested arrays, and 'holes' which allow the analyst to much more easily construct elaborate and detailed system geometries. As with any computer program, the more complicated and elaborate the system under examination, the more CPU time it can be expected to consume. To offset this potential effect, KENO V.a also supports many reflector options, such as mirror reflectors, differential albedo reflectors, and an automatic reflector which has the ability to use reflector region weighting functions which are based on one-dimensional adjoint calculations. The use of these options, typically not only reduces running time, but allows simplified input geometry to be used. In addition to these enhancements, KENO V.a now mixes its own cross sections, making the previously used ICE module unnecessary.

The 27 group cross section library used in this validation was created from a 218 group library developed from ENDF/B-IV data. Both the 27 energy group library and the 16 group Hansen-Roach library are popular for use at Portsmouth, but the 27 group library was chosen for this validation because the origins of the base data used to create it are better documented. The 27 energy group library has a fast group structure similar to the Hansen-Roach library with additional thermal groups, and was collapsed with weighting spectra used to generate the 218 group library. 


\section{VALIDATION DATABASE}

As has been typical of past validation efforts, the weakest portion in this validation is the quality of the critical experiment database. The non-existence of a good quality assured database of critical experiments to validate software against is a problem that has been plaguing the criticality safety community for some time. In the interest of time and manpower, the starting database for this validation was taken from ORNL/CSD/TM-238, the authors of which allowed access to the original TM-238 input cases to alleviate the need to rekey the input data.

The TM-238 validation did not perform an in depth analysis of the critical experiments used unless a blatant abnormality presented itself, and neither will this validation. The authors of TM-238, during their in depth analysis of a few of the cases, did discover definite 'problems' with a few, and subsequently recommended that they be deleted from future validations. This validation study has excluded all those cases.

The input cases are broken down into six major groups, dependent on the specific fissile compound, the enrichment of the fissile isotope, system geometry, and in some cases reflector material.

Appendix B1 contains cases that were all at a $\mathrm{U}^{235}$ enrichment of $4.89 \%$ and were taken from reference 2.0. These include experiments for homogenous single-unit criticals, both reflected and unreflected, as well as heterogenous uranium metal rods in water. The fissile compounds considered were $\mathrm{UO}_{2} \mathrm{~F}_{2}, \mathrm{U}$ metal and $\mathrm{U}_{3} \mathrm{O}_{8}$. The moderating materials were water, sterotex, and $\mathrm{UO}_{2} \mathrm{~F}_{2}$ solution.

Appendix B2 contains cases that were all at a $U^{235}$ enrichment of $3.85 \%$ and were taken from reference 3.0. The experiments represented in these cases are not formally documented at this time. These heterogenous systems consist of large diameter rods which are water moderated, and in some cases the rods have an internal hole which is also water moderated. The critical experiments were modeled in detail from descriptions in experimental logbooks Extensive detail was included in these calculations in order to thoroughly test the KENO V.a geometry package.

Appendix B3 contains cases that include some of the models from reference 4.0 which were used for the validation of Y12CSG for the Oak Ridge Gaseous Diffusion Plant. The validation considered single-unit criticals, both reflected and unreflected, at several enrichments ranging from 1.4 to $4.98 \%$. The first 29 cases consist of $25 \mathrm{UF}_{4}$-paraffin moderated systems and $4 \mathrm{UO}_{2} \mathrm{~F}_{2}$ solution systems at various moderation levels. The last 20 cases are models of $4.46 \%$ enriched damp oxide experiments conducted at Rocky Flats. These damp oxide cases test the codes ability to handle intermixed moderation as well as interstitially moderated systems.

The cases in Appendix B4 consist of highly enriched uranium experiments from reference 2.0. These are $\mathrm{UO}_{2} \mathrm{~F}_{2}$ and $\mathrm{UO}_{2}\left(\mathrm{NO}_{3}\right)_{2}$ solution systems at varying concentrations. Both single-unit and array systems under a variety of reflector conditions are included.

The cases in Appendix B5 include experiments from reference 5.0 which were used for a validation of Y12CSG for the Y-12 plant. Hence identification numbers, assigned by Y-12 personnel are included in the case descriptions. These cases include both single-unit reflected and unreflected systems with uranium metal, uranium alloy, $\mathrm{UO}_{2} \mathrm{~F}_{2}$ solution and $\mathrm{UO}_{2}\left(\mathrm{NO}_{3}\right)_{2}$ solutions. Extensive calculations were performed on arrays, including arrays of metal units with and without interstitial moderation, arrays of $\mathrm{UO}_{2} \mathrm{~F}_{2}$ slab systems, and arrays of 5-liter containers filled with $\mathrm{UO}_{2}\left(\mathrm{NO}_{3}\right)_{2}$ solutions having varying reflector 
thicknesses of paraffin and/or plexiglass.

Appendix B6 is comprised of data gathered during the "eta experiments" conducted at Y-12 around 1960 and documented in reference 6.0. These experiments were of $\mathrm{UO}_{2}\left(\mathrm{NO}_{3}\right)_{2}$ solutions in simple unreflected geometries. The systems were typically very dilute and occasionally included boron poisoning and a few $\mathrm{U}^{233}$ systems.

For ease of comparison, the appendix B numbers in this document correspond to the table numbers used in TM-238. Additionally, the TM-238 case numbers have been retained as well. Of the 258 cases executed to produce TM-238, 13 were excluded from this document; 5 from Table 4 of TM-238, and 8 from Table 5. These cases were excluded on the recommendation of the authors of TM-238 because there were either definite errors found in the modeling of the experiment, duplications of previous experiments, or in one case, not a model of a critical experiment at all.

The results obtained from each of the cases executed, along with a terse description of the model may be found in Appendixes B1 through B6. Additionally, the executed inputs are included in Appendixes E1 through E6. 


\section{STATISTICAL ANALYSIS}

To best determine how to perform an adequate statistical analysis of the data, consultation was held with the statistical analysis department at PORTS. A cursory review of the data revealed it to appear to approximate a normal distribution, as shown in Figure 1. If the data approximates a normal distribution then standard tabulated one-sided tolerance factors for normal distributions may be applied to calculate the minimum acceptable $k_{\text {eff }}$ for the data, $k_{\min }$.

The first step performed on the data was a calculation of the mean, median, and mode. In a perfectly normal distribution, these three values are equivalent. The mean may be calculated from

$$
\bar{x}=\frac{1}{N} \sum_{i=1}^{N} x_{i}
$$

and is simply the arithmetic average.

The median is that value where an equivalent number of values fall both above and below it. In the case of ungrouped data, sorted in ascending order, the value of the median may be taken to be the value of the $(\mathrm{N}+1) / 2$ th item for odd $\mathrm{N}$; for even $\mathrm{N}$ it is customarily taken as the arithmetic average of the (N/2)th item and the $(\mathrm{N} / 2+1)$ th item.

The mode is defined as that value in a distribution about which the values are most heavily concentrated, and may be calculated by the empirical relation:

$$
\text { Mode }=\bar{x}-\sigma\left[\frac{\sqrt{\beta_{1}}\left(\beta_{2}+3\right)}{2\left(5 \beta_{2}-6 \beta_{1}-9\right)}\right]
$$

where:

$$
\begin{array}{ll}
\sigma & =\text { standard deviation about the mean } \\
\sqrt{ } \beta_{1} & =\text { coefficient of skewness } \\
\beta_{2} & =\text { coefficient of kurtosis }
\end{array}
$$

To calculate $\sqrt{ } \beta_{1}$, the coefficient of skewness, and $\beta_{2}$ the coefficient of kurtosis, it is first necessary to calculate the 2 nd, 3rd and 4 th moments about the mean.

$$
\begin{gathered}
M_{r}=\frac{1}{N} \Sigma\left(x_{i}-\bar{x}\right)^{r} \\
I=2,3,4
\end{gathered}
$$

where:

$$
\mathrm{M}_{\mathrm{r}} \quad=\text { rth moment about the mean }
$$


Once $M_{2}, M_{3}$, and $M_{4}$ are obtained,

$$
\sqrt{\beta_{1}}=\frac{M_{3}}{M_{2}^{3 / 2}}
$$

$$
\beta_{2}=\frac{M_{4}}{M_{2}^{2}}
$$

A convenient way at this point to calculate $\sigma$, the standard deviation is

$$
\sigma=\sqrt{M_{2}}
$$

For our data, by the above equation the following values were obtained as moments about the mean.

$$
\begin{aligned}
& M_{2}=1.33698 \times 10^{-4} \\
& M_{3}=6.46422 \times 10^{-7} \\
& M_{4}=7.50532 \times 10^{-8}
\end{aligned}
$$

The mean was then caiculated as

$$
\bar{x}=1.000445183
$$

with

$$
\sigma=\mathrm{k}_{\sigma}=\sqrt{ } \mathrm{M}_{2}=0.011563
$$

The skewness and kurtosis coefficients were respectively

$$
\sqrt{ } \beta_{1}=0.41815 \quad \text { and } \quad \beta_{2}=4.19874 \text {. }
$$

The median was 0.99975 , and the mode, calculated from the above equation as 0.9989 .

Neither the mode, median, or mean are significantly different from each other, as would be expected from normally distributed data.

The skewness coefficient, a measure of the degree of symmetry in a distribution, for a perfectly normal distribution (i.e., or one perfectly symmetric about the mean) would be 0.0 . The kurtosis coefficient, a measure of the peakedness in a distribution, for a perfectly normal distribution would be 3.0 . The value obtained for this analysis 0.41814 and 4.19874 for the skewness and kurtosis coefficient respectively are sufficiently close to those expected from a perfectly normal distribution that it can be considered normally distributed data.

An outlier rejection test was performed, and a few statistically significant outliers were noted. The KENO inputs for these cases were double checked against what was run in TM-238, and the resulting $\mathrm{k}_{\text {eff }}$ values were checked also. Everything was found to be in agreement and the outliers were retained in the data.

It should be noted that the kurtosis coefficient is highly sensitive to the presence of outliers.

Therefore, once a resolution on the outlier cases is obtained, the kurtosis coefficient could be expected 
to improve. It should also be noted that when calculated formally, a tedious process, that the mode of a given set of data is unaffected by the presence of outliers, but when calculated with the given empirical relation, will be affected somewhat by their presence, because the relation is primarily a function of the skewness coefficient and the kurtosis coefficient.

The one-sided tolerance limit for a normal distribution was used to determine the $\mathrm{k}_{\min }$ to be applied to the NCSS system. For a one-sided tolerance limit for a normal distribution, criticality safety personnel are only interested in estimating a value above which a proportion $P$ will lie to a certain confidence level. The value of $k_{\min }$ is then established from

$$
k_{\min }=\bar{k}-K^{*} \sigma
$$

where $\mathrm{K}^{*}$ is the tolerance factor. For $95 \%$ confidence that $99.9 \%$ of the $\mathrm{k}_{\text {eff }}$ values calculated in future calculations will fall above $k_{\min }$ is $K^{*}=3.36136$

$$
\begin{gathered}
\mathrm{k}_{\min }=1.0004452-3.36136(0.011563) \\
\mathrm{k}_{\min }=0.9616
\end{gathered}
$$

To maintain consistency with past operation, the NCS department at PORTS will continue to use $\mathrm{k}_{\min }=$ 0.95 , adding the difference between the two values to the margin of subcriticality.

Bias is defined as the deviation of the calculated values of $\mathbf{k}_{\text {eff }}$ from unity ${ }^{14}$, or an average bias which is taking the absolute value of the difference between an average calculated value of $k_{\text {eff }}$ and unity. For this validation effort, the bias has been established as:

$$
k_{b}=|1-\bar{k}|
$$

which gives $\mathbf{k}_{\mathrm{b}}=0.000445$.

The margin of subcriticality $\left(k_{m}\right)$ can then be calculated based on the following relationship ${ }^{15}$.

$$
k_{\min } \leq 1-k_{b}-1.645 k_{\mathrm{o}}-k_{m}
$$

Which is rearranged to give:

$$
k_{m} \leq 1-k_{b}-1.645 k_{\sigma}-k_{\min }
$$

The incorporation of the 1.645 multiplier on $\mathrm{k}_{\boldsymbol{c}}$ is necessary to place the calculation at the one-sided $95 \%$ confidence level ${ }^{16}$. Substituting then gives:

$$
k_{m} \leq 1-0.000445-1.645(0.011563)-0.95=0.0305
$$

The margin of subcriticality for calculations based on this validation is less than or equal to 0.0305 . 


\section{EXTENSION TO THE K-25 IBM3090}

During the final stages of preparing this validation, personnel at PORTS were notified that the computer used to perform all calculations was scheduled to be permanently shut down in the near future. To alleviate the need to reperform a subsequent validation on a new machine, it was decided that a portion of the required cases would be submitted for processing on the K-25 IBM3090. Approximately 10\% of the inputs were chosen at random. In all cases, the exact same random number sequence was obtained, giving identical results to those obtained on IBM3090 located at X-10.

Therefore, the NCSS software, using the ENDF/B-IV 27 group cross sections is considered valid for use by PORTS personnel on the K-25 IBM3090 as well as the X-10 IBM3090. 


\section{AREA OF APPLICABILITY}

It is considered by PORTS personnel that this validation covers a vast majority of the various fissile systems found at PORTS, and that it is appropriate to use NCSS to analyze them. The different types of low enriched systems include uranium metal at enrichments of $3.85 \%$ and $4.89 \%, \mathrm{H}_{2} \mathrm{O}$ moderated and reflected; $\mathrm{U}_{3} \mathrm{O}_{8}$ stereotex blocks at $4.89 \%$, both $\mathrm{H}_{2} \mathrm{O}$ reflected and unreflected; $\mathrm{UO}_{2} \mathrm{~F}_{2}$ solutions at $4.89 \%$ enriched, with $\mathrm{U}^{235}$ densities ranging from approximately 22.1 to $42.5 \mathrm{gU}^{235} / 1$; and $\mathrm{UF}_{4}$ (paraffin) systems at enrichments from $1.4 \%$ to $3 \%, \mathrm{H} / \mathrm{X}$ ratios from 133 to 971 , reflected and unreflected, to simulate $\mathrm{UF}_{6}$. The highly enriched systems included $\mathrm{UO}_{2} \mathrm{~F}_{2}$ solutions at about $93 \%$, with $\mathrm{H} / \mathrm{X}$ ratios from approximately 76 to 1379 , both reflected and unreflected; $\mathrm{UO}_{2}\left(\mathrm{NO}_{3}\right)_{2}$ solutions at enrichments of $93.2 \%$ with uranium densities ranging from approximately 55 to $364 \mathrm{gU} / 1$, reflected and unreflected; and uranium metal systems between $93 \%$ and $97 \%$, unreflected. A parameterization scheme covering basic system properties such as the average energy group causing fission (AEG), enrichment level, reflector condition, overall system geometry, fuel mixture, etc., was developed and applied to the 245 cases executed. This data may be found in Appendix D and may be used in conjunction with the ranges given above to assist the analyst in determining if a particular system undergoing examination falls within the established area of applicability for this validation

As is, this covers most of the fissile systems at PORTS fairly well. Improvements that could be made include experiments specifically covering oxides at high enrichments, $\mathrm{UF}_{6}$ at high enrichments, and all systems at intermediate enrichments.

PORTS NCS personnel have documentation covering experiments conducted at intermediate enrichments, however it is not known if data covering highly enriched oxide systems and highly enriched $\mathrm{UF}_{6}$ systems exists.

The degree of confidence that can be placed on a calculation when interpolating between experimental data points is typically much higher than can be placed on an extrapolation. The same statement can be made for using calculated data from a computer code validated against experimental data. Prior to applying the minimum $\mathrm{k}_{\mathrm{eff}}$ criteria calculated in this report, the user should verify that the system they are analyzing falls within the established area of applicability for this validation. If it does not, then the minimum $\mathrm{k}_{\text {eff }}$ that will be applied should be lowered accordingly. Without specific information on a system, a recommendation as to how much reduction might be required cannot be made, however Appendix $C$ examines the data segregated into various groups rather than as a collective, and can assist in this determination. The statistical analysis applied in Appendix $C$ takes advantage of the fact that different classes of systems have different overall biases, for example one type of system might on the average be biased $1 \%$ high while another type might be biased $1.3 \%$ low. Clearly, segregating these systems prior to applying a statistical analysis will yield more acceptable results.

In addition to verifying that the system being analyzed falls within the established area of applicability for this validation, the user should also assure themselves that the geometry options they intend to use have been verified by their use at some point in the validation. The 245 cases ran exercised the majority of KENO V.a's geometry options, but do not necessarily exercise all of them. In reference 12.0, NCS personnel at the Paducah Gaseous Diffusion Plant demonstrate that some of the techniques used to reduce running time, such as explicit use of bias, replicate, reflector, and albedos, do not adversely affect the calculated result. This was verified by modeling identical experiments in a variety of ways, and then 
comparing the results. Also, one of the 'convenience' options in KENO V.a, the use of holes, was verified to not adversely affect calculated results, though the use of holes does tend to substantially increase running time. 


\section{RECOMMENDATIONS FOR THE FUTURE}

Aside from the quality of the critical experiment database, the next most significant shortcoming of this validation effort is the grouping of all data into a single group for the statistical analysis and the calculation of a single $k_{\min }$ which is to be applied over the entire range of applicability.

In regard to the quality of the critical experiment database, the original source documents for these experiments should be obtained if they are not already on file. The Nuclear Criticality Safety department at PORTS currently already has the vast majority of the documents referenced in this report. All of the applicable cases should be carefully reviewed, and perhaps modified if data to support the modification is found. Once this verification is completed, thought should be given to then subdividing the experiments into a number of smaller groups, which should be chosen to represent the most common types of fissile systems obtained as PORTS.

One method of dividing the experiments into groups might be based on fissile material enrichment, material composition, system geometry, and unit geometry. Such as was done in Appendix C. Note that these are only examples. Exactly what type scheme is best to employ for this subdivision is still debatable, and it is likely that no one scheme will satisfy all sites due to the vast diversity of operations that exist over multiple sites. Once a suitable subdivision scheme is devised, each group should be closely examined for redundancies and omissions. For example, within any particular group examinations should be conducted to determine if a sufficiently broad range of parameter variation exists to support calculations most required. When omissions, or holes are found, research should be conducted to see if any critical experiment data exists that might be used to make that group more complete. Currently data for intermediate enrichments is rare. It seems that in the past, most critical experiments were conducted at $U^{235}$ enrichments less than $5 \%$, and greater than $90 \%$. Very little data exists for the range in between.

In addition, once the groups are determined, a suitable independent variable should be chosen for the subcritical limit to be calculated against. Currently the independent variable most popular for this is the 'average energy group' causing fission. Though the average energy group causing fission is not an independent variable, there is some merit in choosing it to calculate the subcritical limit against. Once a decision is made, the terminology employed should be carefully examined to avoid contradictions and inadvertent inaccuracies. After this work has been conducted, a "blanket" accepted $k_{\min }$ covering all systems within the area of applicability should no longer be used. The accepted $k_{\min }$ for a particular system should be calculated as a function of the system properties.

Other improvements that could then be made include the development of an objective parameterization scheme that could be applied to a fissile system to aid in establishing if it falls within an established or specified area of applicability, and if not, then how far outside the range it is. Quantifiable data such as that could then be used to calculate how significant a reduction -in $k_{\min }$ would be required to maintain the same degree of safety. 


\section{CONCLUSIONS}

The results of this validation indicate that the NCSS software may be used with confidence for criticality calculations at the Portsmouth Gaseous Diffusion Plant. When the validation results are treated as a single group, there is $95 \%$ confidence that $99.9 \%$ of future calculations of similar critical systems will have a calculated $\mathrm{k}_{\text {eff }}>0.9616$. Based on this result the Portsmouth Nuclear Criticality Safety Department has adopted the calculational acceptance criteria that a $k_{\text {eff }}+2 \sigma \leq 0.95$ is safety subcritical. The validation of NCSS on the IBM 3090 at ORNL was extended to include NCSS on the IBM 3090 at $\mathrm{K}-25$. 


\section{REFERENCES}

W. C. Jordan et all., Validation of KENO V.a Comparison with Critical Experiments, ORNL/CSD/TM-238, Martin Marietta Energy Systems, Inc., Oak Ridge National Laboratory, December 1986

2.0 L. M. Petrie and J. T. Thomas, Assessment of Computational Performance in Nuclear Criticality, ORNL/CSD/TM-224, Martin Marietta Energy Systems, Inc., Oak Ridge National Laboratory, 1985

2.1 E. B. Johnson, The Criticality of Large Uranium Metal Units of Low Enrichment, (to be published at Oak Ridge National Laboratory

2.2 D. F. Cronin, Critical Mass Studies, Part X, ORNL-2968, Union Carbide Nuclear Corporation, Oak Ridge National Laboratory, 1960

J. K. Fox, "Critical Parameters of Uranium Solutions in Simple Geometry," p. 42 in Neutron Physics Division Annual Progress Report for Period Ending Sept 1, 1958, ORNL-2609, Union Carbide Corporation, Nuclear Division, Oak Ridge National Laboratory, 1958

$2.4 \quad$ J. T. Thomas et al, "A Direct Comparison of Some Nuclear Properties of U-233 and U235," Nucl. Sci. Eng. 1, 20, and 32 (1956)

2.5 R. E. Rothe, Benchmark Critical Experiments on High Enriched Uranyl Nitrate Solution Systems, NUREG/CR-0041, RFP-2710, U.S. Nuclear Regulatory Commission, 1978

Davis Reed, Validation of KENO V.a for Critical Lattices of Low Enrichment (3.85\% U235) Uranium Metal Cylinders and Annuli in Water, to be published at ORNL.

3.1 E. B. Johnson, The Criticality of Large Uranium Metal Units of Low Enrichment in U235, to be published at Oak Ridge National Laboratory

4.0 M. E. Easter, Validation of KENO V.a and Two Cross Section Libraries for Criticality Calculations of Low-Enriched Uranium Systems, ORNL/CSD/TM-223, K/HS-74, Martin Marietta Energy Systems, Inc., Oak Ridge National Laboratory, 1985

4.1 F. Abbey, comp, Handbook of Experimental Criticality Data, AHSB(s) Handbook 5, 1968.

S. J. Raffety and J. T. Mihalczo, Homogenized Critical Assemblies of 2 and $3 \%$ Enriched Uranium in Paraffin, Y-DR-14, Union Carbide Corporation, Nuclear Division, Oak Ridge Y-12 Plant, 1969.

E. B. Johnson, "Effect of Steel-Water Reflectors on the Criticality of Low Enriched Uranyl Fluoride Solution," p. 47 in Neutron Physics Division Annual Progress Report for Period Ending May 31, 1968, ORNL-4280, Union Carbide Corporation, Nuclear Division, Oak Ridge National Laboratory, 1968. 
E. B. Johnson, Criticality of a Sphere of U(4.98) $0_{2} \mathrm{~F}_{2}$ Solution, ORNL-3973, Union Carbide Corporation, Nuclear Division, Oak Ridge National Laboratory, 1966, p.14.

4.5 J. W. Webster and E. B. Johnson, Criticality of a Single Unit of Aqueous Uranyl Fluoride Solution Enriched to 5\% in U-235, ORNL-TM-1195, Union Carbide Corporation, Nuclear Division, Oak Ridge National Laboratory, 1965.

R. E. Rothe et al., Critical Experiments with Interstitially Moderated Arrays of Low Enriched Uranium Oxide, NUREG/CR-1071, RFP-3008, U.S. Nuclear Regulatory Commission, 1980.

4.7 G. Tuck and I. Oh, Benchmark Critical Experiments on Low-Enriched Uranium Oxide Systems With $\mathrm{H} / \mathrm{U}=0.77$, NUREG/CR-0674, U.S. Nuclear Regulatory Commission, 1979.

4.8 G. R. Goebel et al., Critical Experiments on Low-Enriched Uranium Oxide Systems With H/U = 1.25, NUREG/CR-1653, RFP-3129, U.S. Nuclear Regulatory Commission, 1980.

R. E. Rothe and G. R. Goebel, Critical Experiments on Low-Enriched Uranium Oxide Systems With H/U = 2.03, NUREG/CR-2500, RFP-3277, U.S. Nuclear Regulatory Commission, 1982.

5.0 J. R. Knight, Validation of the Monte Carlo Criticality Program KENO V.a for Highly Enriched Uranium Systems, ORNL/CSD/TM-221, Martin Marietta Energy Systems, Inc., Oak Ridge National Laboratory, 1984.

5.1 G. E. Hansen, "Properties of Elementary Fast Neutron Critical Assemblies," p. 84 in Proceedings of the Second United Nations International Conference on Peaceful Uses of Atomic Energy, Vol. 12, Geneva, 1958.

C. C. Byers, J. J. Koelling, G. E. Hansen, D. R. Smith, and H. R. Dyer, "Critical Measurements of a Water-Reflected Enriched Uranium Sphere," Trans. Am. Nucl. Soc. 27, 412 (1977).

5.3 R. E. Rothe, Benchmark Critical Experiments on High Enriched Uranyl Nitrate Solution Systems, NUREG/CR-0041, RFP-2710, U.S. Nuclear Regulatory Commission, 1978.

$5.4 \quad$ J. T. Mihalczo and W. E. Kinney, Trans. Am. Nucl. Soc. 4, 302 (1961).

5.5 J. K. Fox, "Critical Parameters of Uranium Solutions in Simple Geometry," p. 42 in Neutron Physics Division Annual Progress Report for Period Ending September 1, 1958, ORNL-2609, Union Carbide Corporation, Nuclear Division, Oak Ridge National Laboratory, 1958.

5.6 Rwin and D. W. Magnuson, Nucl. Sci. Eng. 12, 364 (1962).

5.7 H. C. Paxton, Los Alamos Critical Mass Data, LAMS-3067, Los Alamos National Laboratory, Los Alamos, New Mexico, 1964. 
J. T. Mihalczo, Trans. Am. Nucl. Soc. 8, 442 (1965).

5.9 G. Tuck, "Critical Masses of Spherical and Hemispherical Enriched Uranium Assemblies," J. Nucl. Energy 23, 663 (1969).

D. C. Irving and J. T. Mihalczo, Trans. Am. Nucl. Soc. 7, 287 (1964).

J. T. Thomas, Critical Three-Dimensional Arrays of Neutron-Interacting Units, ORNLTM-719, Union Carbide Corporation, Nuclear Division, Oak Ridge National Laboratory, 1963.

5.12 E. C. Crume and J. T. Thomas, Critical and Near Critical Graphite Moderated Arrays of U(93.2) Cylinders, Y-DD-32, Union Carbide Corporation, Nuclear Division, Oak Ridge Y-12 Plant, 1969.

5.13 B. B. Ernst and C. L. Schuske, Empirical Method for Calculating Pipe Intersections Containing Fissile Solution, RFP-1197, Rockwell International, Rocky Flats, Golden, Colorado, 1968.

5.14 J. T. Thomas, Critical Three-Dimensional Arrays of Neutron-Interacting Units, Part II U(93.2) Metal, ORNL-TM-868, Union Carbide Corporation, Nuclear Division, Oak Ridge National Laboratory, 1964.

5.15 J. K. Fox and L. W. Gilley, Preliminary Report of Critical Experiments in Slab Geometry, ORNL/CF-56-7-148, Union Carbide Corporation, Nuclear Division, Oak Ridge National Laboratory, 1956.

6.0 R. Gwin and D. W. Magnuson, Critical Experiments for Reactor Physics Studies, ORNL/CF-60-4-12, Union Carbide Corporation, Nuclear Division, Oak Ridge National Laboratory, 1960.

7.0 R. Gwin and D. W. Magnuson, "The Measurement of Eta and Other Nuclear Properties of U-233 and U-235 in Critical Aqueous Solutions, Nucl. Sci. Eng. 12, 364-380 (1962).

8.0 H. R. Dyer, "Guide to Preparing the Criticality Safety Evaluation of a Safety Analysis Report for Packaging, NUREG/CR-5661, November 1991.

9.0 G. R. Handley, et al, "Glossary of Nuclear Criticality Safety Terms Used in Safety Analysis Reports at the Y-12 Plant," Y/DD-474, September 4, 1990

10.0 W. Michael Bowen and Carl A. Bennet, Editors, "Statistical Methods for Nuclear Material Management," NUREG/CR-4604, PNL-5849, December 1988

11.0 F. E. Croxton and D. J. Cowden, "Applied General Statistics," New York, PrenticeHall, Inc., 1939.

12.0 T. W. Hines and G. R. Goebel, "Validation of the Oak Ridge National Laboratory Nuclear Criticality Safety Software for 16 and 27 Group Cross-Sections At The Paducah Gaseous Diffusion Plant," KY/E-131, May 1992 
"Martin Marietta Nuclear Criticality Safety Software Users Manual," K/ESH-4, September 1, 1992

14.0 ANSI/ANS-8.1-1983, "Nuclear Criticality Safety in Operations with Fissionable Materials Outside Reactors," American Nuclear Society, October 1983

15.0 DOE-STD-XXXX-93, "DOE Standard: Criticality Safety Program Guide for DOE Nonreactor Nuclear Facilities," PROPOSED, April 1993

16.0 Beyer, W. H. ed., "CRC Standard Mathematical Tables", 27th Edition, CRC Press, 1984 


\section{Appendix A: Calculation of $\boldsymbol{K}$}

Due to the rather large value of $\mathrm{n}$ for this problem, the one-sided tolerance limit $K$ was calculated by a method detailed in reference 10.0 from the equation given below.

$$
K^{*}(n, 1-\alpha, P)=\frac{z_{p}+\sqrt{z_{p}^{2}-a b}}{a}
$$

where:

$$
a=1-\frac{z_{1-\alpha}^{2}}{2(n-1)} \quad b=z_{p}^{2}-\frac{z_{1-\alpha}^{2}}{n}
$$

$Z_{p}$ and $Z_{1-\alpha}$ may be found from the equation

$$
X=\frac{1}{\sqrt{2 \pi}} \int_{-\infty}^{z_{x}} e^{-\frac{1}{2} u^{2}} d u
$$

either numerically or from tables found in most statistics texts.

For this case, $\mathrm{n}=245,1-\alpha=0.95$, and $\mathrm{P}=0.999$, the following values are obtained;

$Z_{1-\alpha}=1.64485, Z_{p}=3.09, a=0.99446$, and $b=9.53706$, which gives $K^{*}=3.36136$. 
Appendix B1: Table 1 Results

\begin{tabular}{|c|c|c|c|}
\hline $\begin{array}{l}\text { PORTS } \\
\text { Case \# }\end{array}$ & $\begin{array}{l}\text { TM-238 } \\
\text { Case \# }\end{array}$ & Experiment Description & $\mathrm{k}_{\mathrm{eff}} \pm \sigma$ \\
\hline 001 & CAA01 & $\begin{array}{l}\text { Experiment } 1 \mathrm{~A} .401-4.89 \% \mathrm{U} \text { rods, } \\
0.762-\mathrm{cm} \text { diam, } 30-\mathrm{cm} \text { long, } 1.3-\mathrm{cm} \\
\text { pitch, } \mathrm{H}_{2} \mathrm{O} \text { moderated and reflected }\end{array}$ & $0.9940 \pm 0.0034$ \\
\hline 002 & $\mathrm{CAAO} 2$ & $\begin{array}{l}\text { Experiment } 4 \mathrm{~B} .400-4.89 \% \mathrm{U} \text { rods, } \\
0.762-\mathrm{cm} \text { diam, } 30-\mathrm{cm} \text { long, } 1.3-\mathrm{cm} \\
\text { pitch, } \mathrm{H}_{2} \mathrm{O} \text { moderated and reflected }\end{array}$ & $0.9922 \pm 0.0031$ \\
\hline 003 & $\mathrm{CAA03}$ & $\begin{array}{l}\text { Experiment } 11 \mathrm{~A} .240-4.89 \% \mathrm{U} \text { rods, } \\
0.762-\mathrm{cm} \text { diam, } 30-\mathrm{cm} \text { long, } 1.3-\mathrm{cm} \\
\text { pitch, } \mathrm{H}_{2} \mathrm{O} \text { moderated and reflected }\end{array}$ & $0.9913 \pm 0.0037$ \\
\hline 004 & CAA04 & $\begin{array}{l}\text { Experiment } 6 \mathrm{~A} .203-4.89 \% \mathrm{U} \text { rods, } \\
0.762-\mathrm{cm} \text { diam, } 30-\mathrm{cm} \text { long, } 2.05-\mathrm{cm} \\
\text { pitch, } \mathrm{H}_{2} \mathrm{O} \text { moderated and reflected }\end{array}$ & $1.0011 \pm 0.0037$ \\
\hline 005 & CAA05 & $\begin{array}{l}\text { Experiment } 8 \mathrm{C} .400-4.89 \% \text { U rods, } \\
0.762-\mathrm{cm} \text { diam, } 30-\mathrm{cm} \text { long, } 2.05-\mathrm{cm} \\
\text { pitch, } \mathrm{H}_{2} \mathrm{O} \text { moderated and reflected }\end{array}$ & $0.9869 \pm 0.0032$ \\
\hline 006 & CAA06 & $\begin{array}{l}\text { Experiment 14A. } 398-4.89 \% \mathrm{U} \text { rods, } \\
0.762-\mathrm{cm} \text { diam, } 30-\mathrm{cm} \text { long, } 1.3-\mathrm{cm} \\
\text { pitch, } \mathrm{H}_{2} \mathrm{O} \text { moderated, } \mathrm{H}_{2} \mathrm{O} \text { top } \\
\text { reflector, Plexiglas bottom reflector, } \\
\text { bottom reflector, } \mathrm{Pb} \text { reflected } 1 \text { face }\end{array}$ & $0.9959 \pm 0.0035$ \\
\hline 007 & CAA07 & $\begin{array}{l}\text { Experiment } 23 \mathrm{~B} .400-4.89 \% \text { U rods, } \\
0.762-\mathrm{cm} \text { diam, } 30-\mathrm{cm} \text { long, } 1.3-\mathrm{cm} \\
\text { pitch, } \mathrm{H}_{2} \mathrm{O} \text { moderated, } \mathrm{H}_{2} \mathrm{O} \text { top } \\
\text { reflector, Plexiglas bottom reflector, } \\
\mathrm{Pb} \text { reflected } 4 \text { faces }\end{array}$ & $0.9937 \pm 0.0034$ \\
\hline 008 & CAA08 & $\begin{array}{l}\text { Experiment 26A. } 215-4.89 \% \text { U rods, } \\
0.762-\mathrm{cm} \text { diam, } 30-\mathrm{cm} \text { long, } 1.3-\mathrm{cm} \\
\text { pitch, } \mathrm{H}_{2} \mathrm{O} \text { moderated, } \mathrm{H}_{2} \mathrm{O} \text { top } \\
\text { reflector, Plexiglas bottom reflector, } \\
\text { Pb reflected } 4 \text { faces }\end{array}$ & $0.9941 \pm 0.0030$ \\
\hline 009 & CAA09 & $\begin{array}{l}\text { Experiment } 28 \mathrm{~A} .255-4.89 \% \mathrm{U} \text { rods, } \\
0.762-\mathrm{cm} \text { diam, } 30-\mathrm{cm} \text { long, } 1.3-\mathrm{cm} \\
\text { pitch, } \mathrm{H}_{2} \mathrm{O} \text { moderated, } \mathrm{H}_{2} \mathrm{O} \text { top }\end{array}$ & $1.0024 \pm 0.0036$ \\
\hline
\end{tabular}


reflector, Plexiglas bottom reflector, $\mathrm{Pb}$ reflected 4 faces, $\mathrm{SS}$ in center

010

CAA10

Experiment 30C. $494-4.89 \%$ U rods, 0.762-cm diam, 30-cm long, 1.3-cm pitch, $\mathrm{H}_{2} \mathrm{O}$ moderated, $\mathrm{H}_{2} \mathrm{O}$ top reflector, Plexiglas bottom reflector, $\mathrm{Pb}$ reflected 4 faces, boral in row 6

011

CAA11

Experiment 31C. $494-4.89 \%$ U rods, 0.762-cm diam, 30-cm long, $1.3-\mathrm{cm}$ pitch, $\mathrm{H}_{2} \mathrm{O}$ moderated, $\mathrm{H}_{2} \mathrm{O}$ top reflector, Plexiglas bottom reflector $\mathrm{Pb}$ reflected 4 faces, $\mathrm{Cd}$ in row 6

012

CAA12

Experiment 32S. $11 \times 12$ array of $4.89 \%$ $\mathrm{U}$ rods in $\sim 300 \mathrm{~g} \mathrm{U} / 1 \mathrm{UO}_{2} \mathrm{~F}_{2}$ solution, $0.762-\mathrm{cm}$ diam, $2.05-\mathrm{cm}$ pitch, in 95.88-cm-diam tank

013

CAA13

Experiment 33S. 9 × 10 array of $4.89 \%$ $\mathrm{U}$ rods in $\sim 300 \mathrm{~g} \mathrm{U} / 1 \mathrm{UO}_{2} \mathrm{~F}_{2}$ solution, $0.762-\mathrm{cm}$ diam, $2.05-\mathrm{cm}$ pitch, in 95.88-cm-diam tank

014

CAA14

Experiment 36S. 9 × 10 array of $4.89 \%$ $\mathrm{U}$ rods in $\sim 300 \mathrm{~g} \mathrm{U} / 1 \mathrm{UO}_{2} \mathrm{~F}_{2}$ solution, 0.762 -cm-diam, 3.25 -cm pitch, in 95.88-cm-diam tank

015

CAA15

Experiment 40S. $6 \times 7$ array of $4.89 \%$ $\mathrm{U}$ rods in $\sim 300 \mathrm{~g} \mathrm{U} / 1 \mathrm{UO}_{2} \mathrm{~F}_{2}$ solution, 1.31-cm-diam, 2.99-cm pitch, in 95.88-cm-diam tank

016

CAA16

Experiment $42 S .6 \times 7$ array of $4.89 \%$ $\mathrm{U}$ rods in $\sim 300 \mathrm{~g} \mathrm{U} / 1 \mathrm{UO}_{2} \mathrm{~F}_{2}$ solution, 1.31-cm-diam, 3.40-cm pitch, in 95.88-cm-diam tank

017

CAA17

Experiment 44S. $6 \times 7$ array of $4.89 \%$ $\mathrm{U}$ rods in $\sim 300 \mathrm{~g} \mathrm{U} / 1 \mathrm{UO}_{2} \mathrm{~F}_{2}$ solution, $1.31-\mathrm{cm}$ diam, 3.94-cm pitch, in 95.88-cm-diam tank 
$\mathrm{U}$ rods in $\sim 300 \mathrm{~g} \mathrm{U} / 1 \mathrm{UO}_{2} \mathrm{~F}_{2}$ solution, 1.31-cm diam, 4.40-cm pitch, in 95.88-cm-diam tank $\mathrm{U}-235 / 1$, unreflected

CAA20

$4.89 \% \mathrm{U}_{3} \mathrm{O}_{8}$ - Stereotex blocks $40.6 \mathrm{~g}$

$1.0041 \pm 0.0029$ $\mathrm{U}-235 / 1(\mathrm{H} / \mathrm{X}=395.0)$, unreflected

021

CAA21

4.89\% $\mathrm{U}_{3} \mathrm{O}_{8}$ - Stereotex blocks, $22.1 \mathrm{~g}$

$0.9997 \pm 0.0033$ $\mathrm{U}-235 / 1(\mathrm{H} / \mathrm{X}=757.0)$, unreflected

022

CAA22

4.89\% $\mathrm{U}_{3} \mathrm{O}_{8}$ - Stereotex blocks $33.3 \mathrm{~g}$ $\mathrm{U}-235 / 1(\mathrm{H} / \mathrm{X}=503.6)$, unreflected

$0.9974 \pm 0.0032$

023

CAA23

$4.89 \% \mathrm{U}_{3} \mathrm{O}_{8}$ - Stereotex blocks $64.91 \mathrm{~g}$

$0.98681 \pm 0.0035$ $\mathrm{U}-235 / 1$ (H/X = 199.3), paraffin top reflector, $\mathrm{H}_{2} \mathrm{O}$ reflected bottom and sides

$4.89 \% \mathrm{U}_{3} \mathrm{O}_{8}$ - Stereotex blocks $56.2 \mathrm{~g}$ $\mathrm{U}-235 / 1(\mathrm{H} / \mathrm{X}=244.8)$, paraffin top reflector, $\mathrm{H}_{2} \mathrm{O}$ reflected bottom and sides $\mathrm{U}-235 / 1(\mathrm{H} / \mathrm{X}=396.7)$, paraffin top reflector, $\mathrm{H}_{2} \mathrm{O}$ reflected bottom and sides $\mathrm{U}-235 / 1(\mathrm{H} / \mathrm{X}=756.5)$, paraffin top reflector, $\mathrm{H}_{2} \mathrm{O}$ reflected bottom and sides

4.89\% $\mathrm{U}_{3} \mathrm{O}_{8}$ - Stereotex blocks $81.1 \mathrm{~g}$ $\mathrm{U}-235 / 1(\mathrm{H} / \mathrm{X}=146.8)$, paraffin top reflector, $\mathrm{H}_{2} \mathrm{O}$ reflected bottom and sides reflector, $\mathrm{H}_{2} \mathrm{O}$ reflected bottom and sides 

reflector, $\mathrm{H}_{2} \mathrm{O}$ reflected bottom and sides

4.89\% $\mathrm{UO}_{2} \mathrm{~F}_{2}$ solution $42.54 \mathrm{~g} \mathrm{U}-235 / 1$

$0.9902 \pm 0.0030$ $(\mathrm{H} / \mathrm{X}=524)$ in a 20 -in.-diam SS cylinder, unreflected $(\mathrm{H} / \mathrm{X}=524)$ in a $20 \times 20$ in. aluminum box, unreflected

$4.89 \% \mathrm{UO}_{2} \mathrm{~F}_{2}$ solution $31.79 \mathrm{~g} \mathrm{U}-235 / 1$ $(\mathrm{H} / \mathrm{X}=735)$ in a 20 -in.-diam SS cylinder, unreflected $(\mathrm{H} / \mathrm{X}=1002)$ in a 27.3-in.-diam aluminum sphere, unreflected

$4.89 \% \mathrm{UO}_{2} \mathrm{~F}_{2}$ solution $24.28 \mathrm{~g} \mathrm{U}-235 / 1$

$1.0010 \pm 0.0028$ $(\mathrm{H} / \mathrm{X}=991)$ in a 30 -in.-diam aluminum cylinder, unreflected

4.89\% $\mathrm{UO}_{2} \mathrm{~F}_{2}$ solution $42.54 \mathrm{~g} \mathrm{U}-235 / 1$ $(\mathrm{H} / \mathrm{X}=524)$ in a 15 -in.-diam SS cylinder, $\mathrm{H}_{2} \mathrm{O}$ reflected

$4.89 \% \mathrm{UO}_{2} \mathrm{~F}_{2}$ solution $42.54 \mathrm{~g} \mathrm{U}-235 / 1$

$(\mathrm{H} / \mathrm{X}=524)$ in a $20 \times 20$ in. aluminum box, $\mathrm{H}_{2} \mathrm{O}$ reflected $(\mathrm{H} / \mathrm{X}=735)$ in a 15 -in.-diam SS cylinder, $\mathrm{H}_{2} \mathrm{O}$ reflected $(\mathrm{H} / \mathrm{X}=991)$ in a 27.3-in.-diam aluminum sphere, $\mathrm{H}_{2} \mathrm{O}$ reflected $(\mathrm{H} / \mathrm{X}=994)$ in a 20 -in.-diam SS cylinder, $\mathrm{H}_{2} \mathrm{O}$ reflected 


\section{Appendix B2: Table 2 Results}

040

041

$\mathrm{CAB02}$

042

$\mathrm{CAB} 03$

043

CAB07

044

CAB08

045

$\mathrm{CAB} 09$

046

CAB10

047
(EBJ.1) lattice 2. 15-3.85\% U rods,

$0.9950 \pm 0.0026$ 7.2 in. diam $\times 30$ in. long in square lattice, 7.2 in. center to center, $77.8 \mathrm{~cm}$ water height

(EBJ.2X) lattice 5. 11-3.85\% U rods, $0.9994 \pm 0.0028$ 7.2 in. diam $\times 30$ in. long in square lattice, 7.95 in. center to center, $72.4 \mathrm{~cm}$ water height

(EBJ.3X) lattice 8. 24-3.85\% U rods, $0.9965 \pm 0.0029$ 7.2 in. diam $\times 30$ in.long in square lattice, 8.70 in. center to center, $75.0 \mathrm{~cm}$ water height

(EBJ.4) lattice 3. 16-3.85\% U rods,

$0.9818 \pm 0.0024$ 7.2 in. diam $\times 30$ in. long in triangular lattice, 7.45 in. center to center, $79.3 \mathrm{~cm}$ water height

(EBJ.5X) lattice 9. 7-3.85\% U rods,

$0.9915 \pm 0.0031$ 7.2 in. diam $\times 30$ in. long in triangular lattice, 8.20 in. center to center, $53.1 \mathrm{~cm}$ water height

(EBJ.6X) lattice 2. 11-3.85\% U rods, $0.9892 \pm 0.0034$ 7.2 in. OD x 2.6 in. ID $\times 30$ in. long in triangular lattice, 7.20 center to center, $77.2 \mathrm{~cm}$ water height

(EBJ.8) lattice 5. 6-3.85\% U rods, $1.0004 \pm 0.0024$ 7.2 in. OD x 2.6 in. ID x 30 in. long in square lattice, 7.95 in. center to center, $91.4 \mathrm{~cm}$ water height ( 2 subcritical)

(EBJ.9) lattice 6. 16-3.85\% U rods, 7.2 in. OD x 2.6 in. ID $\times 30$ in. long in square lattice, 8.70 in. center to center, $49.2 \mathrm{~cm}$ water height 
(EBJ.10) lattice 14. 20-3.85\% U rods,

$0.9979 \pm 0.0027$

7.2 in. $\mathrm{OD} \times 2.6$ in. $\mathrm{D} \times 30$ in. long in square lattice, 9.07 in. center to center, $79 \mathrm{~cm}$ water height

(EBJ.11) lattice 19. 8-3.85\% U rods, 7.2 in. OD $\times 2.6$ in. ID $\times 30$ in. long in square lattice, 7.2 in. center to center, $72.5 \mathrm{~cm}$ water height

050

CAB14

(EBJ.12) lattice 3. 22-3.85\% U rods, $1.0017 \pm 0.0031$ 2.5 in. diam $\times 30$ in. long in square lattice, 2.85 in. center to center, $72.3 \mathrm{~cm}$ water height

051

CAB15

(EBJ.13) lattice 9. 15-3.85\% U rods, $0.9994 \pm 0.0031$ 2.5 in. diam $\times 30$ in. long in square lattice, 3.25 in. center to center, $64.8 \mathrm{~cm}$ water height

052

CAB16

(EBJ.14) lattice 2. 23-3.85\% U rods, $0.9920 \pm 0.0030$ 2.5 in. diam $\times 30$ in. long in square lattice 4.00 in. center to center, $68.9 \mathrm{~cm}$ water height 
Appendix B3: Table 3 Results

053

CAS04 An unreflected rectangular

$0.9854 \pm 0.0023$

parallelepiped of homogeneous

$\mathrm{U}(1.4) \mathrm{F}_{4}$ and paraffin with an

$\mathrm{H} / \mathrm{U}-235$ atomic ratio of $421.8 ; 93.1$

$\mathrm{cm} \times 93.0 \mathrm{~cm} \mathrm{x} 123.8 \mathrm{~cm}$

054

CAS05

An unreflected rectangular

$0.9892 \pm 0.0020$

parallelepiped of homogeneous

$\mathrm{U}(1.4) \mathrm{F}_{4}$ and paraffin with an

$\mathrm{H} / \mathrm{U}-235$ atomic ratio of $421.8 ; 100.0$

$\mathrm{cm} \times 99.9 \mathrm{~cm} \mathrm{x} 103.1 \mathrm{~cm}$

055

CAS06

An unreflected rectangular

$0.9866 \pm 0.0020$

parallelepiped of homogeneous

$\mathrm{U}(1.4) \mathrm{F}_{4}$ and paraffin with an

$\mathrm{H} / \mathrm{U}-235$ atomic ratio of $421.8 ; 130.7$

$\mathrm{cm} \times 130.6 \mathrm{~cm} \times 74.2 \mathrm{~cm}$

056

CAS11

A reflected rectangular parallelepiped

of homogeneous $U(2) F_{4}$ and paraffin with an H/U-235 atomic ratio of 195.2; $56.22 \mathrm{~cm} \mathrm{x} 112.88 \mathrm{~cm}$, reflected with $15.2 \mathrm{~cm}$ of paraffin on top and sides and $15.2 \mathrm{~cm}$ of Plexiglas on the bottom

CAS12

An unreflected rectangular

$0.9924 \pm 0.0024$ parallelepiped of homogeneous $\mathrm{U}(2) \mathrm{F}_{4}$ and paraffin with an $\mathrm{H} / \mathrm{U}-235$ atomic ratio of $195.2 ; 71.47 \mathrm{~cm} \mathrm{x} 71.47 \mathrm{~cm} \mathrm{x}$ $94.14 \mathrm{~cm}$

A reflected rectangular parallelepiped of homogeneous $\mathrm{U}(2) \mathrm{F}_{4}$ and paraffin with an H/U-235 atomic ratio of 293.9; $51.11 \mathrm{~cm} \times 51.11 \mathrm{~cm} \times 73.87 \mathrm{~cm}$, reflected with $15.2 \mathrm{~cm}$ of paraffin on top

An unreflected rectangular parallelepiped of homogeneous $\mathrm{U}(2) \mathrm{F}_{4}$ and paraffin with an $\mathrm{H} / \mathrm{U}-235$ atomic ratio of $293.9 ; 56.22 \mathrm{~cm} \mathrm{x} 56.22 \mathrm{~cm} \mathrm{x}$ $122.47 \mathrm{~cm}$

$0.9951 \pm 0.0025$

$0.9962 \pm 0.0026$

$0.9955 \pm 0.0025$ 
A reflected rectangular parallelepiped

of homogeneous $\mathrm{U}(2) \mathrm{F}_{4}$ and paraffin

with an H/U-235 atomic ratio of 406.3;

$53.67 \mathrm{~cm} \mathrm{x} 53.67 \mathrm{~cm} \mathrm{x} 54.29 \mathrm{~cm}$, reflected with $15.2 \mathrm{~cm}$ of paraffin on top and sides and $15.2 \mathrm{~cm}$ of Plexiglas on the bottom

A reflected rectangular parallelepiped

$0.9964 \pm 0.0023$ of homogeneous $\mathrm{U}(2) \mathrm{F}_{4}$ and paraffin with an H/U-235 atomic ratio of 495.9 ; $46.00 \mathrm{~cm} \mathrm{x} 46.00 \mathrm{~cm} \times 96.57 \mathrm{~cm}$, reflected with $15.2 \mathrm{~cm}$ of paraffin on top and sides and $15.2 \mathrm{~cm}$ of Plexiglas on the bottom

A reflected rectangular parallelepiped of homogeneous $\mathrm{U}(2) \mathrm{F}_{4}$ and paraffin with an H/U-235 atomic ratio of 613.6; $56.32 \mathrm{~cm} \times 61.29 \mathrm{~cm} \times 54.08 \mathrm{~cm}$, reflected with $15.2 \mathrm{~cm}$ of polyethylene on top and sides and $15.2 \mathrm{~cm}$ of Plexiglas on the bottom

An unreflected rectangular parallelepiped of homogeneous $\mathrm{U}(2) \mathrm{F}_{4}$ and paraffin with an $\mathrm{H} / \mathrm{U}-235$ atomic ratio of $613.6 ; 61.3 \mathrm{~cm} \mathrm{x} 66.54 \mathrm{~cm} \mathrm{x}$ $66.52 \mathrm{~cm}$

A reflected rectangular parallelepiped of homogeneous $\mathrm{U}(2) \mathrm{F}_{4}$ and paraffin with an H/U-235 atomic ratio of 971.7; $76.51 \mathrm{~cm} \times 76.44 \mathrm{~cm} \times 82.42 \mathrm{~cm}$, reflected with $5.2 \mathrm{~cm}$ of polyethylene on top and sides and $15.2 \mathrm{~cm}$ of Plexiglas on the bottom

An unreflected rectangular parallelepiped of homogeneous $\mathrm{U}(2) \mathrm{F}_{4}$ and paraffin with an $\mathrm{H} / \mathrm{U}-235$ atomic ratio of $971.7 ; 81.45 \mathrm{~cm} \mathrm{x} 86.70 \mathrm{~cm}$ $x 88.22 \mathrm{~cm}$ of homogeneous $\mathrm{U}(3) \mathrm{F}_{4}$ and paraffin 
with an $\mathrm{H} / \mathrm{U}-235$ atomic ratio of 133.4 ;

$51.14 \mathrm{~cm} \mathrm{x} 51.14 \mathrm{~cm} \times 51.27 \mathrm{~cm}$, reflected with $15.2 \mathrm{~cm}$ of Plexiglas on the bottom

067

CAS22

068

CAS23

069

CAS24

070

CAS25

071

CAS26

072
A reflected rectangular parallelepiped of homogeneous $\mathrm{U}(3) \mathrm{F}_{4}$ and paraffin with an $\mathrm{H} / \mathrm{U}-235$ atomic ratio of 133.4; $43.47 \mathrm{~cm} \mathrm{x} 43.47 \mathrm{~cm} \times 86.39 \mathrm{~cm}$, reflected with $15.2 \mathrm{~cm}$ of Plexiglas on the bottom

A reflected rectangular parallelepiped of homogeneous $\mathrm{U}(3) \mathrm{F}_{4}$ and paraffin with an $\mathrm{H} / \mathrm{U}-235$ atomic ratio of 133.4; $46.02 \mathrm{~cm} \times 46.02 \mathrm{~cm} \times 67.57 \mathrm{~cm}$, reflected with $15.2 \mathrm{~cm}$ of paraffin on top and sides and $15.2 \mathrm{~cm}$ of Plexiglas on the bottom

A reflected rectangular parallelepiped of homogeneous $\mathrm{U}(3) \mathrm{F}_{4}$ and paraffin with an H/U-235 atomic ratio of 133.4; $56.25 \mathrm{~cm} \times 56.25 \mathrm{~cm} \times 43.41 \mathrm{~cm}$, reflected with $15.2 \mathrm{~cm}$ of paraffin on top and sides and $15.2 \mathrm{~cm}$ of Plexiglas on the bottom

A reflected rectangular parallelepiped of homogeneous $\mathrm{U}(3) \mathrm{F}_{4}$ and paraffin with an H/U-235 atomic ratio of 133.4; $61.36 \mathrm{~cm} \times 61.36 \mathrm{~cm} \times 38.67 \mathrm{~cm}$, reflected with $15.2 \mathrm{~cm}$ of paraffin on top and sides and $15.2 \mathrm{~cm}$ of Plexiglas on the bottom

An unreflected rectangular parallelepiped of homogeneous $\mathrm{U}(3) \mathrm{F}_{4}$ and paraffin with an $\mathrm{H} / \mathrm{U}-235$ atomic ratio of $133.4 ; 56.47 \mathrm{~cm} \mathrm{x} 56.47 \mathrm{~cm} \mathrm{x}$ $86.64 \mathrm{~cm}$

An unreflected rectangular parallelepiped of homogenous $\mathrm{U}(3) \mathrm{F}_{4}$ and paraffin with an $\mathrm{H} / \mathrm{U}-235$ atomic ratio of $133.4 ; 56.25 \mathrm{~cm} \mathrm{x} 61.36 \mathrm{~cm}$
$1.0038 \pm 0.0029$

$1.0055 \pm 0.0037$ 
$\mathrm{x} 74.38 \mathrm{~cm}$

073

CAS28

An unreflected rectangular

$1.0066 \pm 0.0030$ parallelepiped of homogeneous $\mathrm{U}(3) \mathrm{F}_{4}$ and paraffin with an $\mathrm{H} / \mathrm{U}-235$ atomic ratio of $133.4 ; 61.4 \mathrm{~cm} \mathrm{x} 61.4 \mathrm{~cm} \mathrm{X}$ $66.0 \mathrm{~cm}$

074

CAS29

075

CAS30

076

CAS31

077

CAS32

078

CAS33

079
A reflected rectangular parallelepiped of homogeneous $\mathrm{U}(3) \mathrm{F}_{4}$ and paraffin with an H/U-235 atomic ratio of 276.9; $40.81 \mathrm{~cm} \mathrm{x} 40.80 \mathrm{~cm} \times 39.49 \mathrm{~cm}$, reflected with $15.2 \mathrm{~cm}$ of polyethylene on top and sides and $15.2 \mathrm{~cm}$ of Plexiglas on the bottom

An unreflected rectangular parallelepiped of homogeneous $\mathrm{U}(3) \mathrm{F}_{4}$ and paraffin with an $\mathrm{H} / \mathrm{U}-235$ atomic ratio of $276.9 ; 40.90 \mathrm{~cm} \mathrm{x} 40.93 \mathrm{~cm} \mathrm{x}$ $116.80 \mathrm{~cm}$

An unreflected rectangular parallelepiped of homogeneous $\mathrm{U}(3) \mathrm{F}_{4}$ and paraffin with an $\mathrm{H} / \mathrm{U}-235$ atomic ratio of $276.9 ; 48.59 \mathrm{~cm} \mathrm{x} 51.14 \mathrm{~cm} \mathrm{X}$ $48.53 \mathrm{~cm}$

An unreflected rectangular parallelepiped of homogeneous $\mathrm{U}(3) \mathrm{F}_{4}$ and paraffin with an $\mathrm{H} / \mathrm{U}-235$ atomic ratio of $276.9 ; 81.71 \mathrm{~cm} \mathrm{x} 81.66 \mathrm{~cm} \mathrm{x}$ $31.34 \mathrm{~cm}$

A composite cadmium/steel/water side reflected stainless steel cylinder of $0.079 \mathrm{~cm}$ wall thickness and $19.545 \mathrm{~cm}$ IR filled to a height of $54.45 \mathrm{~cm}$ with $\mathrm{U}(4.98) \mathrm{O}_{2} \mathrm{~F}_{2}$ solution at an $\mathrm{H} / \mathrm{U}-235$ atomic ratio of 488

A composite 1-in. steel/water side reflected steel cylinder of $0.079 \mathrm{~cm}$ wall thickness and $16.51 \mathrm{~cm}$ IR filled to a height of $143 \mathrm{~cm}$ with $\mathrm{U}(4.98) \mathrm{O}_{2} \mathrm{~F}_{2}$ solution at an $\mathrm{H} / \mathrm{U}-235$ atomic ratio of
$1.0040 \pm 0.0029$

$1.0109 \pm 0.0028$

$1.0043 \pm 0.0029$

$1.0128 \pm 0.0031$

$0.9952 \pm 0.0029$

$0.9992 \pm 0.0028$

\section{B-10}



with an H/U-235 atomic ratio of 490 .

Solution radius of $25.3873 \mathrm{~cm}$ and stainless steel container wall thickness of $0.0508 \mathrm{~cm}$

An unreflected stainless steel

$0.9955 \pm 0.0032$ cylinder of $0.07874 \mathrm{~cm}$ wall thickness and a $19.55 \mathrm{~cm} \mathrm{IR}$ filled to a height of $101.7 \mathrm{~cm}$ with $\mathrm{U}(4.98) \mathrm{O}_{2} \mathrm{~F}_{2}$ solution at an H/U-235 atomic ratio of 496

Experiment 1. $4.46 \%$ enriched $\mathrm{U}_{3} \mathrm{O}_{8}$, $\mathrm{H} / \mathrm{U}=0.77,42$ fuel cans with $2.44 \mathrm{~cm}$ interstitial moderation, plastic reflected $\mathrm{H} / \mathrm{U}=0.77,43$ fuel cans with $2.44 \mathrm{~cm}$ interstitial moderation, plastic reflected $\mathrm{H} / \mathrm{U}=0.77,100$ fuel cans with 0.929 cm interstitial moderation, plastic reflected

Experiment 13. $4.46 \%$ enriched $\mathrm{U}_{3} \mathrm{O}_{8}$, $1.0169 \pm 0.0032$ $\mathrm{H} / \mathrm{U}=0.77,40$ fuel cans with $2.44 \mathrm{~cm}$ interstitial moderation, concrete reflected

Experiment $15.4 .46 \%$ enriched $\mathrm{U}_{3} \mathrm{O}_{8}$, $\mathrm{H} / \mathrm{U}=0.77,98$ fuel cans with 0.929 $\mathrm{cm}$ interstitial moderation, concrete reflected driven by $93.12 \%$ enriched uranium metal sphere $(29.870 \mathrm{~kg}), 120+4 \mathrm{~S}$ fuel cans, plastic reflected 
$\mathrm{kg} 351.18 \mathrm{~g} / \mathrm{l})$ fuel cans, plastic

reflected

089

CAR08

4.46\% enriched $\mathrm{U}_{3} \mathrm{O}_{8} \mathrm{H} / \mathrm{U}=0.77$, driven by low concentration (12.871

$\mathrm{kg} 86.42 \mathrm{~g} / \mathrm{l}) 93.17 \%$ enriched

$\mathrm{UO}_{2}\left(\mathrm{NO}_{3}\right)_{2}, 119+2 \mathrm{~S}$ fuel cans, plastic reflected

090

CAR09

091

CAR10

092

CAR11

093

CAR12

094

CAR13

095

CAR14

096

4.46\% enriched $\mathrm{U}_{3} \mathrm{O}_{8}, \mathrm{H} / \mathrm{U}=0.77$,

driven by low concentration (13.001

$\mathrm{kg} 86.42 \mathrm{~g} / \mathrm{l}) 93.17 \%$ enriched

$\mathrm{UO}_{2}\left(\mathrm{NO}_{3}\right)_{2}, 119+2 \mathrm{~S}$ fuel cans, plastic reflected

$4.46 \%$ enriched $\mathrm{U}_{3} \mathrm{O}_{8}, \mathrm{H} / \mathrm{U}=0.77$,

driven by low concentration (12.446

$\mathrm{kg} 86.42 \mathrm{~g} / \mathrm{l})$ 93.17\% enriched

$\mathrm{UO}_{2}\left(\mathrm{NO}_{3}\right)_{2}, 119+2 \mathrm{~S}$ fuel cans, concrete reflected

Experiment A. $4.46 \%$ enriched $\mathrm{U}_{3} \mathrm{O}_{8}$

$\mathrm{H} / \mathrm{U}=1.25,38$ fuel cans with $2.44 \mathrm{~cm}$ interstitial moderation, plastic

reflected

Experiment B. $4.46 \%$ enriched $\mathrm{U}_{3} \mathrm{O}_{8}$

$\mathrm{H} / \mathrm{U}=1.25,78$ fuel cans with 0.929

$\mathrm{cm}$ interstitial moderation, plastic

reflected

Experiment C. $4.46 \%$ enriched $\mathrm{U}_{3} \mathrm{O}_{8}$

$\mathrm{H} / \mathrm{U}=1.25,80$ fuel cans with $0.929 \mathrm{~cm}$ interstitial moderation, plastic reflected by high concentration $(12.268 \mathrm{~kg}$ $351.64 \mathrm{gU} / \mathrm{l}), 93.17 \%$ enriched

$\mathrm{UO}_{2}\left(\mathrm{NO}_{3}\right)_{2}, 119+2 \mathrm{~S}$ fuel cans, plastic reflected

4.46\% enriched $\mathrm{U}_{3} \mathrm{O}_{8} \mathrm{H} / \mathrm{U}=1.25$, driven

$0.9941 \pm 0.0034$

$0.9949 \pm 0.0035$

$1.0072 \pm 0.0034$

$1.0166 \pm 0.0031$

$1.0155 \pm 0.0033$

$4.46 \%$ enriched $\mathrm{U} 308 \mathrm{H} / \mathrm{U}=1.25$, driven $1.0073 \pm 0.0029$

$0.9968 \pm 0.0035$ by high concentration $(12.400 \mathrm{~kg}$ $351.65 \mathrm{gU} / \mathrm{l})$, 93.17\% enriched $\mathrm{UO}_{2}\left(\mathrm{NO}_{3}\right)_{2}, 119+2 \mathrm{~S}$ fuel cans, plastic 
reflected

097

CAR16

4.46\% enriched $\mathrm{U}_{3} \mathrm{O}_{8} \mathrm{H} / \mathrm{U}=1.25$ driven

$1.0018 \pm 0.0031$

by low concentration $(10.836 \mathrm{~kg} \mathrm{p}$

$86.60 \mathrm{gU} / \mathrm{l}), 93.17 \%$ enriched

$\mathrm{UO}_{2}\left(\mathrm{NO}_{3}\right)_{2}, 119+2 \mathrm{~S}$ fuel cans,

plastic reflected

098

CAR17

Experiment F. $4.46 \%$ enriched $\mathrm{U}_{3} \mathrm{O}_{8}$

$1.0035 \pm 0.0031$

$\mathrm{H} / \mathrm{U}=2.03,48$ fuel cans with 0.92

cm interstitial moderation, plastic

reflected

099

CAR18

Experiment G. $4.46 \%$ enriched $\mathrm{U}_{3} \mathrm{O}_{8}$

$1.0025 \pm 0.0031$ $\mathrm{H} / \mathrm{U}=2.03,30$ fuel cans with $2.44 \mathrm{~cm}$ interstitial moderation, plastic reflected

100

CAR19

Experiment D. $4.46 \%$ enriched $\mathrm{U}_{3} \mathrm{O}_{8}$ $\mathrm{H} / \mathrm{U}=2.03$, driven by $93.12 \%$ enriched hollow uranium metal sphere $(13.73 \mathrm{~kg})$, $120+4 S$ fuel cans, plastic reflected

101

CAR20

Experiment E. $4.46 \%$ enriched $\mathrm{U}_{3} \mathrm{O}_{8}$ $\mathrm{H} / \mathrm{U}=2.03$, driven by $93.12 \%$ enriched hollow uranium metal sphere $(12.786$ $\mathrm{kg}), 120+4 \mathrm{~S}$ fuel cans, plastic reflected 
Appendix B4: Table 4 Results

102

103

104

105

106

107

113

114

115

116

117

118

119
CAA01 $\mathrm{U}(93.2) \mathrm{O}_{2} \mathrm{~F}_{2}$ solution sphere $\mathrm{H} / \mathrm{X}=$ 1112 , unreflected

CAA02 $U(93.2) \mathrm{O}_{2} \mathrm{~F}_{2}$ solution sphere $\mathrm{H} / \mathrm{X}=$ 1393, unreflected

CAA03 $\quad \mathrm{U}(93.2) \mathrm{O}_{2}\left(\mathrm{NO}_{3}\right)_{2}$ solution sphere

$\mathrm{H} / \mathrm{X}=1379$, unreflected

CAA04 U(93.2) $\mathrm{O}_{2} \mathrm{~F}_{2}$ solution sphere $\mathrm{H} / \mathrm{X}=$ $76.1, \mathrm{H}_{2} \mathrm{O}$ reflected

CAA05 U(93.2) $\mathrm{O}_{2} \mathrm{~F}_{2}$ solution sphere $\mathrm{H} / \mathrm{X}=$ $126.5, \mathrm{H}_{2} \mathrm{O}$ reflected

CAA06 $\mathrm{U}(93.2) \mathrm{O}_{2} \mathrm{~F}_{2}$ solution sphere $\mathrm{H} / \mathrm{X}=$ $1270, \mathrm{H}_{2} \mathrm{O}$ reflected

CAA12 U(93.2) $\mathrm{O}_{2}\left(\mathrm{NO}_{3}\right)_{2}$ solution $142.92 \mathrm{~g}$

$\mathrm{U} / 1,28.01 \mathrm{~cm}$ diam cylinder, unreflected

CAA13

$\mathrm{U}(93.2) \mathrm{O}_{2}\left(\mathrm{NO}_{3}\right)_{2}$ solution $357.71 \mathrm{~g}$ $\mathrm{U} / \mathrm{l}, 28.01 \mathrm{~cm}$ diam cylinder, unreflected

CAA14

$\mathrm{U}(93.2) \mathrm{O}_{2}\left(\mathrm{NO}_{3}\right)_{2}$ solution $54.89 \mathrm{~g}$

$\mathrm{U} / \mathrm{l}, 33.01 \mathrm{~cm}$ diam cylinder, unreflected

CAA15 U(93.2) $\mathrm{O}_{2}\left(\mathrm{NO}_{3}\right)_{2}$ solution $137.4 \mathrm{~g}$

$\mathrm{U} / \mathrm{l}, 33.01 \mathrm{~cm}$ diam cylinder, unreflected

CAA16

$\mathrm{U}(93.2) \mathrm{O}_{2}\left(\mathrm{NO}_{3}\right)_{2}$ solution $357.71 \mathrm{~g}$

$\mathrm{U} / \mathrm{l}, 33.01 \mathrm{~cm}$ diam cylinder, unreflected

CAA17

$\mathrm{U}(93.2) \mathrm{O}_{2}\left(\mathrm{NO}_{3}\right)_{2}$ solution $144.38 \mathrm{~g}$

$\mathrm{U} / \mathrm{l}, 28.01 \mathrm{~cm}$ diam cylinder, concrete reflected

CAA18

$\mathrm{U}(93.2) \mathrm{O}_{2}\left(\mathrm{NO}_{3}\right)_{2}$ solution $334.77 \mathrm{~g}$

$1.0109 \pm 0.0028$

$1.0067 \pm 0.0022$

$0.9950 \pm 0.0023$

$1.0120 \pm 0.0042$

$1.0031 \pm 0.0041$

$1.0050 \pm 0.0024$

$1.0015 \pm 0.0047$

$1.0211 \pm 0.0042$

$1.0112 \pm 0.0032$

$1.0032 \pm 0.0045$

$1.0054 \pm 0.0046$

$1.0086 \pm 0.0043$

$1.0112 \pm 0.0046$ 
$\mathrm{U} / \mathrm{l}, 28.01 \mathrm{~cm}$ diam cylinder, concrete reflected

$\mathrm{U} / \mathrm{l}, 33.01 \mathrm{~cm}$ diam cylinder, concrete reflected

$\mathrm{U}(93.2) \mathrm{O}_{2}\left(\mathrm{NO}_{3}\right)_{2}$ solution $334.77 \mathrm{~g}$. $\mathrm{U} / \mathrm{l}, 33.01 \mathrm{~cm}$ diam cylinder, concrete reflected

$\mathrm{U} / \mathrm{l}, 28.01 \mathrm{~cm}$ diam cylinder, concrete reflected reflected reflected

$\mathrm{U}\left(93.2 \mathrm{OO}_{2}\left(\mathrm{NO}_{3}\right)_{2}\right.$ solution $345.33 \mathrm{~g}$ $\mathrm{U} / \mathrm{l}, 28.01 \mathrm{~cm}$ diam cylinder, Plexiglas reflected $33.01 \mathrm{~cm}$ diam cylinder, Plexiglas reflected $33.01 \mathrm{~cm}$ diam cylinder, Plexiglas reflected $28.01 \mathrm{~cm}$ diam cylinder, Plexiglas reflected $33.01 \mathrm{~cm}$ diam cylinder, Plexiglas reflected $21.12 \mathrm{~cm}$ diam cylinder in a $4 \times 4$ array, concrete reflected 

$\mathrm{U} / \mathrm{l}, 21.12 \mathrm{~cm}$ diam cylinder in a $4 \times 4$ array, concrete reflected $16.12 \mathrm{~cm}$ diam cylinder in a $4 \times 4$ array, concrete reflected

$\mathrm{U}(93.2) \mathrm{O}_{2}\left(\mathrm{NO}_{3}\right)_{2}$ solution $359.55 \mathrm{~g}$ $1.0079 \pm 0.0041$ $\mathrm{U} / \mathrm{l}, 16.12 \mathrm{~cm}$ diam cylinder in a $4 \times 4$ array, concrete reflected

$\mathrm{U}(93.2) \mathrm{O}_{2}\left(\mathrm{NO}_{3}\right)_{2}$ solution $76.09 \mathrm{~g} \mathrm{U} / 1$, $1.0100 \pm 0.0034$ $21.12 \mathrm{~cm}$ diam cylinder in a $2 \times 2$ array, concrete reflected

$\mathrm{U}(93.2) \mathrm{O}_{2}\left(\mathrm{NO}_{3}\right)_{2}$ solution $364.11 \mathrm{~g}$ $\mathrm{U} / \mathrm{l}, 21.2 \mathrm{~cm}$ diam cylinder in a $2 \times 2$ array, concrete reflected

$\mathrm{U}(93.2) \mathrm{O}_{2}\left(\mathrm{NO}_{3}\right)_{2}$ solution $359.55 \mathrm{~g}$ $1.0063 \pm 0.0040$ $\mathrm{U} / 1,16.12 \mathrm{~cm}$ diam cylinder in a $2 \times 2$ array, concrete reflected

$\mathrm{U}(93.2) \mathrm{O}_{2}\left(\mathrm{NO}_{3}\right)_{2}$ solution $359.55 \mathrm{~g}$ $\mathrm{U} / \mathrm{l}, 16.12 \mathrm{~cm}$ diam cylinder in a $2 \times 4$ array, concrete reflected $21.12 \mathrm{~cm}$ diam cylinder in a $4 \times 4$ array, Plexiglas reflected $\mathrm{U} / 1,21.12 \mathrm{~cm}$ diam cylinder in a $4 \times 4$ array, Plexiglas reflected $16.12 \mathrm{~cm}$ diam cylinder in a $4 \times 4$ array, Plexiglas reflected

$\mathrm{U}(93.2) \mathrm{O}_{2}\left(\mathrm{NO}_{3}\right)_{2}$ solution $355.94 \mathrm{~g}$ $\mathrm{U} / \mathrm{l}, 16.12 \mathrm{~cm}$ diam cylinder in a $4 \times 4$ array, Plexiglas reflected 
array, Plexiglas reflected

143

CAA42 U(93.2) $\mathrm{O}_{2}\left(\mathrm{NO}_{3}\right)_{2}$ solution $355.94 \mathrm{~g}$

$1.0086 \pm 0.0039$

$\mathrm{U} / \mathrm{l}, 21.12 \mathrm{~cm}$ diam cylinder in a

$2 \times 2$ array, Plexiglas reflected

144

CAA43

$\mathrm{U}(93.2) \mathrm{O}_{2}\left(\mathrm{NO}_{3}\right)_{2}$ solution $355.94 \mathrm{~g}$

$\mathrm{U} / 1,16.12 \mathrm{~cm}$ diam cylinder in a

$1.0006 \pm 0.0041$

$3 \times 2$ array, Plexiglas reflected 
Appendix B5: Table 5 Results

145

148

149

150

151

152

153

154

155

146

147

156
CAS01

CAS04

CAS05

CAS06

CAS07

CAS08

CAS09

CAS10

CAS11

CAS02

CASO3

CAS12
$\mathrm{Y}-12$ validation case $\mathrm{A}-1.93 .8 \% \mathrm{U}$

metal sphere, unreflected (GODIVA)

Y-12 validation case A-2. 93.2\% U-Mo

alloy cylinder annulus, unreflected

$\mathrm{Y}-12$ validation case $\mathrm{A}-3.93 .2 \% \mathrm{UO}_{2} \mathrm{~F}_{2}$ solution, $19.992 \mathrm{~g} \mathrm{U} / \mathrm{l}$, in Al sphere, unreflected

Y-12 validation case $A-4.93 .18 \%$

$\mathrm{UO}_{2}\left(\mathrm{NO}_{3}\right)_{2}$ solution, $20.12 \mathrm{~g} \mathrm{U} / \mathrm{l}$, in

$\mathrm{Al}$ sphere, unreflected

Y-12 validation case A-5. 93.5\% U

metal hemispherical shell, $\mathrm{H}_{2} \mathrm{O}$

reflected

Y-12 validation case A-6. 93.2\% U

metal cylinder annulus, graphite

reflected

Y-12 validation case $A-7.94 \% U$ metal cuboid, natural U reflected

Y-12 validation case A-8. 93.1\% U

metal hemispherical shell, oil

reflected

Y-12 validation case A-9. 93.1\%. U

metal hemispherical shell, steel

center and oil reflected

Y-12 validation case A-10. 97.67\% U metal sphere, $\mathrm{H}_{2} \mathrm{O}$ reflected

$\mathrm{Y}-12$ validation case $\mathrm{A}-11.93 .172 \%$ $\mathrm{UO}_{2}\left(\mathrm{NO}_{3}\right)_{2}$ solution, $346.7 \mathrm{~g} \mathrm{U} / 1$, in SS cylinder, unreflected
$1.0002 \pm 0.0030$

$0.9999 \pm 0.0029$

$1.0025 \pm 0.0024$

$1.0016 \pm 0.0022$

$1.0112 \pm 0.0034$

$1.0159 \pm 0.0034$

$1.0038 \pm 0.0032$

$1.0101 \pm 0.0032$

$1.0014 \pm 0.0033$

$1.0016 \pm 0.0030$

$1.0155 \pm 0.0046$

$0.9952 \pm 0.0030$

$\mathrm{Y}-12$ validation case $\mathrm{B}-1.93 .2 \% \mathrm{U}$ metal cylinder annulus, unreflected, smaller $U$ cylinder in hole touching 
one wall

CAS22 Y-12 validation case B-2. 93.2\% U

$1.0032 \pm 0.0027$ metal cylinder annulus, unreflected, smaller $U$ block in hole touching one wall metal, unreflected cylinders and cuboids in approximate circular arrangement, cylinder, cuboid, and hemisphere stack in center metal cylinders, $4 \times 4 \times 4$ array, unreflected metal cylinders, $2 \times 2 \times 2$ array, each unit in the array is a smaller cylinder capped on each end by a larger cylinder, unreflected metal cylinders, $2 \times 2 \times 2$ array, paraffin reflected metal cylinders, $2 \times 2 \times 2$ array, each unit in the array is a smaller cylinder capped on each end by a larger cylinder, paraffin reflected metal cylinders each in a Plexiglas box, $2 \times 2 \times 2$ array of these units unreflected cylinders, $4 \times 4$ array, standing in a solution slab, Plexiglas reflected 
reflected

Y-12 validation case B-16. 93.1\%

$1.0242 \pm 0.0042$

$\mathrm{UO}_{2}\left(\mathrm{NO}_{3}\right)_{2}$ solution, $450.8 \mathrm{~g} \mathrm{U} / 1$, in SS containers, square central column with 8 perpendicular cylindrical arms unreflected

Problem S333SPO. 93.2\% $\mathrm{UO}_{2} \mathrm{~F}_{2}$ solution, $1.0015 \pm 0.0040$ $81.8 \mathrm{~g} \mathrm{U} / 1$, in Al slabs, $37.62 \mathrm{~cm}$ slabs in $3 \times 1$ array, $0 \mathrm{~cm}$ separation, unreflected, cylindrical tank, floor and walls in experiment room included

Problem S333SPI. 93.2\% $\mathrm{UO}_{2} \mathrm{~F}_{2}$ solution, $81.8 \mathrm{~g} \mathrm{U} / 1$, in Al slabs, $37.62 \mathrm{~cm}$ slabs in $3 \times 1$ array, $2.54 \mathrm{~cm}$ separation, unreflected, cylindrical tank, floor, and walls in experiment room included

Problem S333SP3. 93.2\% $\mathrm{UO}_{2} \mathrm{~F}_{2}$ solution, $81.8 \mathrm{~g} \mathrm{U} / \mathrm{l}$, in $\mathrm{Al}$ slabs, $37.62 \mathrm{~cm}$ slabs in $3 \times 1$ array, $7.62 \mathrm{~cm}$ separation, unreflected, cylindrical tank, floor, and walls in experiment room included

Problem S333SP4. 93.2\% UO $\mathrm{UF}_{2}$ solution, $81.8 \mathrm{~g} \mathrm{U} / 1$, in $\mathrm{Al}$ slabs, $37.62 \mathrm{~cm}$ slabs in $3 \times 1$ array, $11.43 \mathrm{~cm}$ separation, unreflected, cylindrical tank, floor, and walls in experiment room included

Problem S333SP5. 93.2\% UO $\mathrm{UO}_{2}$ solution, $81.8 \mathrm{~g} \mathrm{U} / 1$, in $\mathrm{Al}$ slabs, $37.62 \mathrm{~cm}$ slabs in $3 \times 1$ array, $13.97 \mathrm{~cm}$ separation, unreflected, cylindrical tank, floor, and walls in experiment room included

Problem S333SP6. 93.2\% UO $\mathrm{UF}_{2}$ solution, $81.8 \mathrm{~g} \mathrm{U} / \mathrm{l}$, in $\mathrm{Al}$ slabs, $37.62 \mathrm{~cm}$ slabs in $3 \times 1$ array, $15.24 \mathrm{~cm}$ separation, unreflected, cylindrical

$0.9936 \pm 0.0038$ 
tank, floor, and walls in experiment room included

177

179

CAS35

181

CAS37

183

CAS39

186

185

CAS41

187

188

CAS33

\author{
CAS35
}

CAS37

CAS39

CAS42

CAS41

187

CAS43

CAS44
Problem S333SPOR. 93.2\% $\mathrm{UO}_{2} \mathrm{~F}_{2}$ solution, $81.8 \mathrm{~g} \mathrm{U} / 1$, in Al slabs, $37.62 \mathrm{~cm}$ slabs in $3 \times 1$ array, $0 \mathrm{~cm}$ separation, $\mathrm{H}_{2} \mathrm{O}$ reflected

Problem S333SP1R. 93.2\% UO $\mathrm{U}_{2}$ solution, $81.8 \mathrm{~g} \mathrm{U} / 1$, in $\mathrm{Al}$ slabs $37.62 \mathrm{~cm}$ slabs in $3 \times 1$ array, 2.54 cm separation, $\mathrm{H}_{2} \mathrm{O}$ reflected

Problem S333SP3R. 93.2\% $\mathrm{UO}_{2} \mathrm{~F}_{2}$ solution, $81.8 \mathrm{~g} \mathrm{U} / 1$, in Al slabs, $37.62 \mathrm{~cm}$ slabs in $3 \times 1$ array, 7.62 cm separation, $\mathrm{H}_{2} \mathrm{O}$ reflected

Problem S333SP4R. 93.2\% UO $\mathrm{UO}_{2}$ solution, $81.8 \mathrm{~g} \mathrm{U} / 1$, in Al slabs, $37.62 \mathrm{~cm}$ slabs in $3 \times 1$ array, $11.43 \mathrm{~cm}$ separation, $\mathrm{H}_{2} \mathrm{O}$ reflected

Problem S333SP5R. 93.2\% UO $\mathrm{U}_{2}$ solution, $81.8 \mathrm{~g} \mathrm{U} / 1$, in $\mathrm{Al}$ slabs, $37.62 \mathrm{~cm}$ slabs in $3 \times 1$ array, $13.97 \mathrm{~cm}$ separation, $\mathrm{H}_{2} \mathrm{O}$ reflected

Problem S36SP2. 93.2\% UO $\mathrm{UF}_{2}$ solution, $\quad 0.9816 \pm 0.0042$ $81.8 \mathrm{~g} \mathrm{U} / 1$ in Al slabs, $7.62 \mathrm{~cm}$ and $14.834 \mathrm{~cm}$ slabs in $2 \times 1$ array, 5.08 cm separation, unreflected

Problem S36SP15. 93.2\% UO $\mathrm{UO}_{2}$ solution, $81.8 \mathrm{~g} \mathrm{U} / 1$ in $\mathrm{Al}$ slabs, $7.62 \mathrm{~cm}$ and $14.834 \mathrm{~cm}$ slabs in $2 \times 1$ array, 38.1 $\mathrm{cm}$ separation, unreflected

Problem S36SP30. 93.2\% UO $\mathrm{UO}_{2}$ solution, $81.8 \mathrm{~g} \mathrm{U} / \mathrm{l}$ in Al slabs, $7.62 \mathrm{~cm}$ and $14.834 \mathrm{~cm}$ slabs in $2 \times 1$ array, 76.2 $\mathrm{cm}$ separation, unreflected

Problem S36SP48. 93.2\% UO $\mathrm{UO}_{2}$ solution, $81.8 \mathrm{~g} \mathrm{U} / \mathrm{l}$ in Al slabs, $7.62 \mathrm{~cm}$ and $14.834 \mathrm{~cm}$ slabs in $2 \times 1$ array, 121.92
$0.9886 \pm 0.0040$

$0.9953 \pm 0.0036$

$0.9903 \pm 0.0035$

$1.0048 \pm 0.0033$

$1.0030 \pm 0.0035$

$0.9790 \pm 0.0042$

$0.9690 \pm 0.0042$

$0.9827 \pm 0.0037$ 
cm separation, unreflected

Problem S363SPO. 93.2\% UO $\mathrm{UF}_{2}$ solution,

$0.9846 \pm 0.0038$

$81.8 \mathrm{~g} \mathrm{U} / \mathrm{l}$, in Al slabs, $7.62 \mathrm{~cm}$, $14.834 \mathrm{~cm}$, and $7.62 \mathrm{~cm}$ slabs in $3 \times 1$

array, $0 \mathrm{~cm}$ separation, unreflected

CAS46

Problem S363SP10. 93.2\% $\mathrm{UO}_{2} \mathrm{~F}_{2}$

$0.9868 \pm 0.0043$ solution, $81.8 \mathrm{~g} \mathrm{U} / 1$, in Al slabs, $7.62 \mathrm{~cm}, 14.834 \mathrm{~cm}$, and $7.62 \mathrm{~cm}$ slabs in $3 \times 1$ array, $25.4 \mathrm{~cm}$ separation, unreflected

Problem S363SP20. 93.2\% $\mathrm{UO}_{2} \mathrm{~F}_{2}$ solution, $81.8 \mathrm{~g} \mathrm{U} / \mathrm{l}$, in $\mathrm{Al}$ slabs, $7.62 \mathrm{~cm}, 14.834 \mathrm{~cm}$, and $7.62 \mathrm{~cm}$ slabs in $3 \times 1$ array, $50.8 \mathrm{~cm}$ separation, unreflected

Problem S363SP32. 93.2\% UO $\mathrm{UO}_{2}$ solution, $81.8 \mathrm{~g} \mathrm{U} / 1$, in Al slabs, $7.62 \mathrm{~cm}, 14.834 \mathrm{~cm}$, and $7.62 \mathrm{~cm}$ slabs in $3 \times 1$ array, $81.28 \mathrm{~cm}$ separation, unreflected $81.8 \mathrm{~g} \mathrm{U} / 1$, in Al slabs. One slab is made up from two $7.62 \mathrm{~cm}$ slabs snugly fit together, the other is $7.62 \mathrm{~cm}$, $2 \times 1$ array, $15.24 \mathrm{~cm}$ separation, unreflected

Problem S63SP12. 93.2\% $\mathrm{UO}_{2} \mathrm{~F}_{2}$ solution, $0.9705 \pm 0.0032$ $81.8 \mathrm{~g} \mathrm{U} / \mathrm{l}$, in Al slabs. One slab is made up from two $7.62 \mathrm{~cm}$ slabs snugly fit together, the other is $7.62 \mathrm{~cm}$, $2 \times 1$ array, $30.48 \mathrm{~cm}$ separation, unreflected $81.8 \mathrm{~g} \mathrm{U} / \mathrm{l}$, in Al slabs. One slab is made up from two $7.62 \mathrm{~cm}$ slabs snugly fit together, the other is $7.62 \mathrm{~cm}$, $2 \times 1$ array, $45.72 \mathrm{~cm}$ separation, unreflected 
$81.8 \mathrm{~g} \mathrm{U} / \mathrm{l}$, in Al slabs. One slab is made up from two $7.62 \mathrm{~cm}$ slabs snugly

fit together, the other is $7.62 \mathrm{~cm}$, $2 \times 1$ array, $76.2 \mathrm{~cm}$ separation, unreflected

198

CAS54

202

CAS58

197

CAS53

199

200

201
CAS56

CAS55

CAS56

CAS57

Problem S66SP2. 93.2\% $\mathrm{UO}_{2} \mathrm{~F}_{2}$ solution, $0.9855 \pm 0.0045$ $81.8 \mathrm{~g} \mathrm{U} / 1$, in Al slabs. One slab is $14.834 \mathrm{~cm}$, and the other is made up from two $7.62 \mathrm{~cm}$ slabs snugly fit together, $2 \times 1$ array, $5.08 \mathrm{~cm}$ separation, unreflected

Problem S66SP2. 93.2\% UO $\mathrm{F}_{2}$ solution, $\quad 0.9834 \pm 0.0039$ $81.8 \mathrm{~g} \mathrm{U} / 1$, in Al slabs. One slab is $14.834 \mathrm{~cm}$, and the other is made up from two $7.62 \mathrm{~cm}$ slabs snugly fit together, $2 \times 1$ array, $15.24 \mathrm{~cm}$ separation, unreflected

Problem S66SP15. 93.2\% UO $\mathrm{UF}_{2}$ solution, $\quad 0.9881 \pm 0.0034$ $81.8 \mathrm{~g} \mathrm{U} / 1$, in $\mathrm{Al}$ slabs. One slab is $14.834 \mathrm{~cm}$, and the other is made up from two $7.62 \mathrm{~cm}$ slabs snugly fit together, $2 \times 1$ array, $38.1 \mathrm{~cm}$ separation, unreflected

Problem S66SP20. 93.2\% UO $\mathrm{UO}_{2}$ solution, $\quad 0.9903 \pm 0.0038$ $81.8 \mathrm{~g} \mathrm{U} / 1$, in Al slabs. One slab is $14.834 \mathrm{~cm}$, and the other is made up from two $7.62 \mathrm{~cm}$ slabs snugly fit together, $2 \times 1$ array, $50.8 \mathrm{~cm}$ separation, unreflected

Problem S66SP30. 93.2\% UO $\mathrm{F}_{2}$ solution, $81.8 \mathrm{~g} \mathrm{U} / \mathrm{l}$, in Al slabs. One slab is $14.834 \mathrm{~cm}$, and the other is made up from two $7.62 \mathrm{~cm}$ slabs snugly fit together, $2 \times 1$ array, $76.2 \mathrm{~cm}$ separation, unreflected

$0.9921 \pm 0.0036$

Problem S66SP48. 93.2\% UO $\mathrm{UF}_{2}$ solution, $0.9927 \pm 0.0041$ $81.8 \mathrm{~g} \mathrm{U} / 1$, in $\mathrm{Al}$ slabs. One slab is $14.834 \mathrm{~cm}$, and the other is made up from two $7.62 \mathrm{~cm}$ slabs snugly fit 
together, $2 \times 1$ array, $121.92 \mathrm{~cm}$

separation, unreflected

Problem S66SP66. 93.2\% $\mathrm{UO}_{2} \mathrm{~F}_{2}$ solution,

$0.9867 \pm 0.0032$ $81.8 \mathrm{~g} \mathrm{U} / \mathrm{l}$, in Al slabs. One slab is $14.834 \mathrm{~cm}$, and the other is made up from two $7.62 \mathrm{~cm}$ slabs snugly fit together, $2 \times 1$ array, $167.64 \mathrm{~cm}$ separation, unreflected

Problem U6B271F. 92.6\% UO $\mathrm{UO}_{2}\left(\mathrm{NO}_{3}\right)_{2}$

$1.0025 \pm 0.0039$ solution, $63.3 \mathrm{~g} \mathrm{U} / 1$, in Plexiglas cylinders, $3 \times 3 \times 3$ array, unreflected, walls, floor, and tank in experiment room included

Problem U2B271F. 92.6\% UO $\left(\mathrm{NO}_{3}\right)_{2}$ solution, $279 \mathrm{~g} \mathrm{U} / \mathrm{l}$, in Plexiglas cylinders, $3 \times 3 \times 3$ array, unreflected, walls, floor, and tank in experiment room included

Problem U2B81F. 92.6\% UO $\left(\mathrm{NO}_{3}\right)_{2}$ solution, $279 \mathrm{~g} \mathrm{U} / \mathrm{l}$, in Plexiglas cylinders, $2 \times 2 \times 2$ array, unreflected, walls, floor, and tank in experiment room included

Problem U4B1251F. 92.6\% UO $\mathrm{UO}_{2}\left(\mathrm{NO}_{3}\right)_{2}$ solution, $415 \mathrm{~g} \mathrm{U} / 1$, in Plexiglas cylinders, $5 \times 5 \times 5$ array, unreflected, walls, floor, and tank in experiment room included

Problem U4B641F. 92.6\% UO $\left(\mathrm{NO}_{3}\right)_{2}$ solution, $415 \mathrm{~g} \mathrm{U} / 1$, in Plexiglas cylinders, $4 \times 4 \times 4$ array, unreflected, walls, floor, and tank in experiment room included

Problem U4B271F. 92.6\% UO $\mathrm{UN}_{2}\left(\mathrm{NO}_{3}\right)_{2}$ solution, $415 \mathrm{~g} \mathrm{U} / \mathrm{l}$, in Plexiglas cylinders, $3 \times 3 \times 3$ array, unreflected, walls, floor, and tank in experiment room included 
Problem U4B81F. 92.6\% UO $\left(\mathrm{NO}_{3}\right)_{2}$

$0.9989 \pm 0.0046$

solution, $415 \mathrm{~g} \mathrm{U} / 1$, in Plexiglas

cylinders, $2 \times 2 \times 2$ array, unreflected, walls, floor, and tank in experiment room included

Problem U4U2B27. 92.6\% UO $\mathrm{UO}_{2}\left(\mathrm{NO}_{3}\right)_{2}$

$1.0035 \pm 0.0044$ solution, $415 \mathrm{~g} \mathrm{U} / \mathrm{l}$, in Plexiglas cylinders, $3 \times 3 \times 3$ array, unreflected, $279 \mathrm{~g} \mathrm{U} / 1$ in 5 central units, walls, floor, and tank in experiment room included

Problem U4R27A1F. 92.6\% $\mathrm{UO}_{2}\left(\mathrm{NO}_{3}\right)_{2}$ solution, $415 \mathrm{~g} \mathrm{U} / \mathrm{l}$, in Plexiglas cylinders, $3 \times 3 \times 3$ array, reflected, $15.24 \mathrm{~cm}$ paraffin on bottom, $1.27 \mathrm{~cm}$ Plexiglas on other faces

Problem U4R27B1F. 92.6\% $\mathrm{UO}_{2}\left(\mathrm{NO}_{3}\right)_{2}$ $1.0169 \pm 0.0036$ solution, $415 \mathrm{~g} \mathrm{U} / \mathrm{l}$, in Plexiglas cylinders, $3 \times 3 \times 3$ array, reflected, $15.24 \mathrm{~cm}$ paraffin on bottom, $2.54 \mathrm{~cm}$ Plexiglas on other faces

Problem U4R27C1F. 92.6\% UO $\left(\mathrm{NO}_{3}\right)_{2}$ solution, $415 \mathrm{~g} \mathrm{U} / \mathrm{l}$, in Plexiglas cylinders, $3 \times 3 \times 3$ array, reflected, $15.24 \mathrm{~cm}$ paraffin on bottom, $1.27 \mathrm{~cm}$ Plexiglas on other faces

Problem U4R27D1F. 92.6\% UO $\left(\mathrm{NO}_{3}\right)_{2}$ solution, $415 \mathrm{~g} \mathrm{U} / \mathrm{l}$, in Plexiglas cylinders, $3 \times 3 \times 3$ array, reflected, $15.24 \mathrm{~cm}$ paraffin on 5 faces, $15.24 \mathrm{~cm}$ Plexiglas on 1 face

Problem U4R27E1F. 92.6\% UO $\left(\mathrm{NO}_{3}\right)_{2}$ solution, $415 \mathrm{~g} \mathrm{U} / 1$, in Plexiglas cylinders, $3 \times 3 \times 3$ array, reflected, $15.24 \mathrm{~cm}$ paraffin on bottom, $3.81 \mathrm{~cm}$ paraffin on other faces

Problem U4R27F1F. 92.6\% UO $\mathrm{UO}_{2}\left(\mathrm{NO}_{3}\right)_{2}$ solution, $415 \mathrm{~g} \mathrm{U} / \mathrm{l}$, in Plexiglas cylinders, $3 \times 3 \times 3$ array, reflected, 
$15.24 \mathrm{~cm}$ paraffin on bottom, $7.62 \mathrm{~cm}$ paraffin on other faces

CAS72

217

CAS73

219

CAS75

220

CAS76

221

CAS77

222

CAS78

223

CAS79

224

Problem U4R27G1F. $92.6 \% \quad \mathrm{UO}_{2}\left(\mathrm{NO}_{3}\right)_{2}$ solution, $415 \mathrm{~g} \mathrm{U} / 1$, in Plexiglas cylinders $3 \times 3 \times 3$ array, reflected, $1.27 \mathrm{~cm}$ Plexiglas all faces

Problem U4R27H1F. 92.6\% UO $\left(\mathrm{NO}_{3}\right)_{2}$

solution, $415 \mathrm{~g} \mathrm{U} / 1$, in Plexiglas

cylinders, $3 \times 3 \times 3$ array, reflected, $1.27 \mathrm{~cm}$ paraffin all faces

Problem U4R27J1F. 92.6\% UO $\left(\mathrm{NO}_{3}\right)_{2}$ solution, $415 \mathrm{~g} \mathrm{U} / \mathrm{l}$, in Plexiglas cylinders, $3 \times 3 \times 3$ array, reflected, $3.81 \mathrm{~cm}$ paraffin all faces

Problem U4R8A1F. 92.6\% UO $\left(\mathrm{NO}_{3}\right)_{2}$ solution, $415 \mathrm{~g} \mathrm{U} / \mathrm{l}$, in Plexiglas cylinders, $2 \times 2 \times 2$ array, reflected, $15.24 \mathrm{~cm}$ paraffin on bottom, $1.27 \mathrm{~cm}$ Plexiglas on other faces

Problem U4R8B1F. 92.6\% UO $\left(\mathrm{NO}_{3}\right)_{2}$ solution, $415 \mathrm{~g} \mathrm{U} / \mathrm{l}$, in Plexiglas cylinders, $2 \times 2 \times 2$ array, reflected, $15.24 \mathrm{~cm}$ paraffin on bottom, $11.43 \mathrm{~cm}$ Plexiglas on other faces

Problem U4R8C1F. 92.6\% UO $\left(\mathrm{NO}_{3}\right)_{2}$ solution, $415 \mathrm{~g} \mathrm{U} / \mathrm{l}$, in Plexiglas cylinders, $2 \times 2 \times 2$ array, reflected, $15.24 \mathrm{~cm}$ paraffin on bottom, $15.24 \mathrm{~cm}$ Plexiglas on other faces

Problem U4R8D1F. 92.5\% UO $\left(\mathrm{NO}_{3}\right)_{2}$ solution, $415 \mathrm{~g} \mathrm{U} / 1$, in Plexiglas cylinders, $2 \times 2 \times 2$ array, reflected, $15.24 \mathrm{~cm}$ paraffin on bottom, $2.54 \mathrm{~cm}$ Plexiglas on other faces

Problem U4R8E1F. $92.6 \% \mathrm{UO}_{2}\left(\mathrm{NO}_{3}\right)_{2}$

$0.9971 \pm 0.0043$

$1.0142 \pm 0.0046$

$1.0272 \pm 0.0041$

$1.0143 \pm 0.0042$ $1.0252 \pm 0.0040$ $1.0278 \pm 0.0039$ $1.0285 \pm 0.0039$ solution, $415 \mathrm{~g} \mathrm{U} / \mathrm{l}$, in Plexiglas cylinders, $2 \times 2 \times 2$ array, reflected, $15.24 \mathrm{~cm}$ paraffin on bottom, $4.45 \mathrm{~cm}$ 
Plexiglas on other faces

Problem U4R8G1F. 92.6\% UO $\mathrm{UO}_{2}\left(\mathrm{NO}_{3}\right)_{2}$

$1.0214 \pm 0.0039$

solution, $415 \mathrm{~g} \mathrm{U} / \mathrm{l}$, in Plexiglas

cylinders, $2 \times 2 \times 2$ array, reflected, $15.24 \mathrm{~cm}$ paraffin on bottom, $6.35 \mathrm{~cm}$

Plexiglas on other faces

CAS82

Problem U4R8G1F. 92.6\% UO $\mathrm{UN}_{3} \mathrm{NO}_{2}$

$1.0183 \pm 0.0041$ solution, $415 \mathrm{~g} \mathrm{U} / \mathrm{l}$, in Plexiglas cylinders, $2 \times 2 \times 2$ array, reflected, $15.24 \mathrm{~cm}$ paraffin on bottom, $1.27 \mathrm{~cm}$ Plexiglas on other faces

Problem U4R8H1F. 92.6\% UO $\left(\mathrm{NO}_{3}\right)_{2}$ $1.0169 \pm 0.0037$ solution, $415 \mathrm{~g} \mathrm{U} / \mathrm{l}$, in Plexiglas cylinders, $2 \times 2 \times 2$ array, reflected, $15.24 \mathrm{~cm}$ paraffin on bottom, $3.81 \mathrm{~cm}$ Plexiglas on other faces

Problem U4R8I1F. 92.6\% UO $\mathrm{UO}_{2}\left(\mathrm{NO}_{3}\right)_{2}$ solution, $415 \mathrm{~g} \mathrm{U} / \mathrm{l}$, in Plexiglas cylinders, $2 \times 2 \times 2$ array, reflected, $15.24 \mathrm{~cm}$ paraffin on bottom, $7.62 \mathrm{~cm}$ Plexiglas on other faces

Problem U4R8J1F. 92.6\% UO $\left(\mathrm{NO}_{3}\right)_{2}$ solution, $415 \mathrm{~g} \mathrm{U} /$, in Plexiglas cylinders, $2 \times 2 \times 2$ array, reflected $1.27 \mathrm{~cm}$ Plexiglas all faces

Problem U4R8K1F. 92.6\% $\mathrm{UO}_{2}\left(\mathrm{NO}_{3}\right)_{2}$ solution, $415 \mathrm{~g} \mathrm{U} / \mathrm{l}$, in Plexiglas cylinders, $2 \times 2 \times 2$ array, reflected, $1.27 \mathrm{~cm}$ paraffin all faces

Problem U4R8L1F. 92.6\% UO $\left(\mathrm{NO}_{3}\right)_{2}$ solution, $415 \mathrm{~g} \mathrm{U} / 1$, in Plexiglas cylinders, $2 \times 2 \times 2$ array, reflected, $15.24 \mathrm{~cm}$ paraffin all faces $3.81 \mathrm{~cm}$ paraffin all faces 
Problem U4R8N1F. 92.6\% UO $\mathrm{UN}_{2}\left(\mathrm{NO}_{3}\right)_{2}$ solution, $415 \mathrm{~g} \mathrm{U} / \mathrm{l}$, in Plexiglas cylinders, $2 \times 2 \times 2$ array, reflected, $7.62 \mathrm{~cm}$ paraffin all faces 


\section{Appendix B6: Table 6 Inputs}

236

237

238

239

240

241

242

243

244

245

246

247

248
CAE01

CAE02

CAE03

CAE04

CAE05

CAE06

CAE07

CAE08

CAE09

CAE10

CAE11

CAE12

CAE13
Experiment 1. U(93.2) $\mathrm{O}_{2}\left(\mathrm{NO}_{3}\right)_{2} \mathrm{H} / \mathrm{X}=$ 1378 in 27.24-in. diam sphere

Experiment 2. U(93.2) $\mathrm{O}_{2}\left(\mathrm{NO}_{3}\right)_{2} \mathrm{H} / \mathrm{X}=$ 1177 , B poisoned, in 27.24-in. diam sphere

Experiment 3. U(93.2) $\mathrm{O}_{2}\left(\mathrm{NO}_{3}\right)_{2} \mathrm{H} / \mathrm{X}=$ 1033, B poisoned, in 27.24-in. diam sphere

Experiment 4. U(93.2) $\mathrm{O}_{2}\left(\mathrm{NO}_{3}\right)_{2} \mathrm{H} / \mathrm{X}=$ 972, B poisoned, in 27.24-in. diam sphere

Experiment 5. U-233 $\mathrm{O}_{2}\left(\mathrm{NO}_{3}\right)_{2} \mathrm{H} / \mathrm{X}=$ 1533 , in 27.24-in. diam sphere

Experiment 6. U-233 $\mathrm{O}_{2}\left(\mathrm{NO}_{3}\right)_{2} \mathrm{H} / \mathrm{X}=$ 1470, B poisoned, in 27.24-in. diam sphere

Experiment 7. U-233 $\mathrm{O}_{2}\left(\mathrm{NO}_{3}\right)_{2} \mathrm{H} / \mathrm{X}=$ 1417, B poisoned, in 27.24-in. diam sphere

Experiment 8. U-233 $\mathrm{O}_{2}\left(\mathrm{NO}_{3}\right)_{2} \mathrm{H} / \mathrm{X}=$ 1368 , B poisoned, in 27.24-in. diam sphere

Experiment 9. U-233 $\mathrm{O}_{2}\left(\mathrm{NO}_{3}\right)_{2} \mathrm{H} / \mathrm{X}=$ 1835 , in 48.04-in. diam sphere

Experiment 10. U(93.2) $\mathrm{O}_{2}\left(\mathrm{NO}_{3}\right)_{2} \mathrm{H} / \mathrm{X}=$ 1835 , in 48.04-in. diam sphere

Experiment 11. U-233 $\mathrm{O}_{2}\left(\mathrm{NO}_{3}\right)_{2} \mathrm{H} / \mathrm{X}=$ 1986, in 48.04-in. diam sphere

Experiment 12. U(93.2) $\mathrm{O}_{2}\left(\mathrm{NO}_{3}\right)_{2} \mathrm{H} / \mathrm{X}=$ 1604 , in 60.92-in. diam cylinder

Experiment 13. $\mathrm{U}(93.2) \mathrm{O}_{2}\left(\mathrm{NO}_{3}\right)_{2} \mathrm{H} / \mathrm{X}=$
$0.9925 \pm 0.0024$

$0.9991 \pm 0.0025$

$0.9912 \pm 0.0022$

$1.0002 \pm 0.0023$

$1.0034 \pm 0.0024$

$0.9999 \pm 0.0026$

$1.0070 \pm 0.0023$

$0.9994 \pm 0.0024$

$1.0075 \pm 0.0023$

$0.9921 \pm 0.0016$

$0.9930 \pm 0.0018$

$0.9953 \pm 0.0019$

$0.9959 \pm 0.0018$ 
1634, in 60.92-in. diam cylinder

CAE14 Experiment 14. U(93.2) $\mathrm{O}_{2}\left(\mathrm{NO}_{3}\right)_{2} \mathrm{H} / \mathrm{X}=$

$0.9979 \pm 0.0018$

1821 , in 60.92-in. diam cylinder

250

CAE15

Experiment 15. $\mathrm{U}(93.2) \mathrm{O}_{2}\left(\mathrm{NO}_{3}\right)_{2} \mathrm{H} / \mathrm{X}=$

$0.9982 \pm 0.0017$

1905, in 60.92-in. diam cylinder

251

CAE16

Experiment 16. U(93.2) $\mathrm{O}_{2}\left(\mathrm{NO}_{3}\right)_{2} \mathrm{H} / \mathrm{X}=$ 1981 , in 60.92-in. diam cylinder

252

CAE17 Experiment 17. U-233 $\mathrm{O}_{2}\left(\mathrm{NO}_{3}\right)_{2} \mathrm{H} / \mathrm{X}=$ 1819 , in 60.92-in. diam cylinder

253

CAE18

Experiment 18. U-233 $\mathrm{O}_{2}\left(\mathrm{NO}_{3}\right)_{2} \mathrm{H} / \mathrm{X}=$ 1900 , in 60.92-in. diam cylinder

254

CAE19

Experiment 19. U-233 $\mathrm{O}_{2}\left(\mathrm{NO}_{3}\right)_{2} \mathrm{H} / \mathrm{X}=$ 1996, in 60.92-in. diam cylinder

255

CAE20

Experiment 20. U-233 $\mathrm{O}_{2}\left(\mathrm{NO}_{3}\right)_{2} \mathrm{H} / \mathrm{X}=$ 2106 , in 60.92-in. diam cylinder

256

CAE21

Experiment 21. U(93.2) $\mathrm{O}_{2}\left(\mathrm{NO}_{3}\right)_{2} \mathrm{H} / \mathrm{X}=$ 1955 , in 107.7-in. diam cylinder

257

CAE22

Experiment 22. U(93.2) $\mathrm{O}_{2}\left(\mathrm{NO}_{3}\right)_{2} \mathrm{H} / \mathrm{X}=$ 2004, in 107.7-in. diam cylinder

CAE23

Experiment 23. $\mathrm{U}(93.2) \mathrm{O}_{2}\left(\mathrm{NO}_{3}\right)_{2} \mathrm{H} / \mathrm{X}=$ 2052, in 107.7-in. diam cylinder 


\section{Appendix C: Statistical Analysis Based on Segregated Groups}

Additional statistical analyses of the validation results were performed based on the data being subdivided into various groups. In these analyses the data were evaluated with respect to a correlation variable over the range of that variable. The treatment of high and low enriched experiments together as a group may not be appropriate due to the large range of enrichments which have been considered in the validation. The effect of treating enrichment as an independent variable is discussed. In addition, the validation cases were grouped by enrichment into highly enriched critical experiments $\left(U_{235}\right.$ enrichments $>90 \%$ ) and low enriched critical experiments ( $U^{235}$ enrichments $<5 \%$ ) and evaluated using the average energy group of the neutron causing fission (AEG) as the independent variable. An evaluation was also performed in which the data were grouped by table number, as in TM-238, and evaluated using AEG as the independent variable.

The validation experiments were parameterized by average energy group of the neutron causing fission, enrichment level, fuel enrichment, reflection conditions, reflector material, system geometry, unit geometry, fuel mixture and form, and fissile material. These data are presented in Appendix D. The tables provided in Appendix D may be used to determine the applicability of the validation to a specific system being analyzed.

The mean $\mathrm{k}_{\mathrm{eff}}$ and standard deviation of the mean for each grouping of data considered are shown in Table C.1. Also presented are estimates of the lower $95 \%$ confidence bound of $\mathrm{k}_{\text {eff }}$ and the lower $95 \%$ confidence on $99.9 \%$ of future calculations bound of $k_{\text {eff }}$ when the data are treated as a group. The lower $95 \%$ confidence bound of $\mathrm{k}_{\mathrm{eff}}$ may be interpreted as that value of $\mathrm{k}_{\mathrm{eff}}$ that one would expect to calculate above, $95 \%$ of the time, if the system being analyzed is a critical system. The lower $95 \%$ confidence on $99.9 \%$ of future calculations bound may be interpreted as the value of $k_{\text {eff }}$ that, for a critical system similar to those validated, one would calculate above for $99.9 \%$ of the estimates of $k_{\text {eff }}$ at the $95 \%$ confidence level. These values will be discussed in more detail later.

In the establishment of the confidence bounds of $\mathrm{k}_{\mathrm{eff}}$, the variability of the KENO calculations has been included in the estimate of the total standard deviation. This is appropriate in the general case because, while each estimate of $k_{e f}$ is that of a critical system, each estimate is a single estimate of a different physical arrangement of fissile material which has imbedded an individual uncertainty. In the specific case where all of the estimates of $k_{\text {eff }}$ are for a specific system or very similar systems, this treatment will overestimate the variability and be slightly conservative.

The results presented in Table C.1 indicate that if the validation data is treated as a group, a calculated $\mathrm{k}_{\text {eff }}$ of less than 0.95 would be considered subcritical at the $95 \%$ confidence level. With the exception of the Table 5 grouping and the Table 5 - high AEG grouping, a calculated $\mathrm{k}_{\text {eff }}$ of less than 0.95 would be considered as subcritical $99.9 \%$ of the time at the $95 \%$ confidence level. The Table 5 results have a large total standard deviation due to the bias in a significant number of the validation experiment groups (eg. the 1F arrays - cases CAS62-CAS91 have a positive bias of about 1-1/2\%, and the slab experiments - cases CAS29-CAS58 have a negative bias of about 1\%). 
Table C.1: Statistical estimates for grouped data

\begin{tabular}{|c|c|c|c|c|c|c|c|c|c|}
\hline erouping & $\begin{array}{l}\text { mamber of } \\
\text { observations }\end{array}$ & man & $\begin{array}{l}\text { oumberd } \\
\text { deviation }\end{array}$ & $\begin{array}{l}\text { vanime } \\
\text { vature }\end{array}$ & 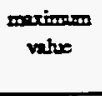 & $\underbrace{}_{\text {variex }}$ & $\begin{array}{l}\text { Lotel stonderd } \\
\text { deviation }\end{array}$ & $\begin{array}{l}\text { bower } \\
95 \% \\
\text { bound }\end{array}$ & $\begin{array}{l}\text { bower } 95 \% \\
\text { bound on } \\
99.9 \times\end{array}$ \\
\hline Nll anes & 245 & 1.0004 & $1.1587-2$ & 0.9686 & 1.0510 & 1.12445 & $1.2062-2$ & 0.9806 & 0.9599 \\
\hline $\begin{array}{l}\text { bow enrichod } \\
\text { asere }\end{array}$ & 101 & 0.9971 & $8.2925-3$ & 0.9686 & 1.0169 & 8.8757 .6 & $8.8113-3$ & 0.9825 & 0.9659 \\
\hline $\begin{array}{l}\text { hich } \\
\text { carrided } \\
\text { calces }\end{array}$ & 144 & 1.0028 & 1.2945 .2 & 0.9600 & 1.0510 & $1.2501-5$ & $1.3434-2$ & 0.9807 & 0.9564 \\
\hline $\begin{array}{c}\text { Table } 1 \\
\text { anese }\end{array}$ & 39 & 0.9937 & 7.1189 .3 & 0.9686 & 1.0045 & 9.236366 & $7.7405-3$ & 0.8806 & 0.9635 \\
\hline $\begin{array}{c}\text { Table } 2 \\
\text { arcas }\end{array}$ & 12 & 0.9957 & $6.0732-3$ & 0.9818 & 1.0048 & $8.2878-6$ & $6.7210-3$ & 0.9837 & 0.9623 \\
\hline $\begin{array}{c}\text { Table } 3 \\
\text { caser }\end{array}$ & 49 & 1.0002 & $8.5860-3$ & 0.9818 & 1.0169 & $8.7417-6$ & $9.0810-3$ & 0.9850 & 0.9659 \\
\hline $\begin{array}{c}\text { Table } 4 \\
\text { casces }\end{array}$ & 38 & 1.0069 & $5.8675-3$ & 0.9910 & 1.0211 & $1.5427-5$ & $7.0608-3$ & 0.9950 & 0.9793 \\
\hline $\begin{array}{c}\text { Table } 5 \\
\text { casce }\end{array}$ & 83 & 1.0026 & $1.5929-3$ & 0.9650 & 1.0510 & 1.4233-5 & $1.6370-2$ & 0.9753 & 0.9438 \\
\hline $\begin{array}{l}\text { Table } 5 \text { bow } \\
\text { AEG asea }\end{array}$ & 17 & 1.0041 & $7.4530-3$ & 0.9931 & 1.0159 & $9.5368-6$ & $8.0675-3$ & 0.9900 & 0.9673 \\
\hline $\begin{array}{l}\text { Table S hith } \\
\text { AEG cosed }\end{array}$ & 66 & 1.0022 & 1.7248 .2 & 0.9690 & 1.0510 & $1.5443-5$ & $1.7919-2$ & 0.9723 & 0.9365 \\
\hline $\begin{array}{c}\text { Table } 6 \\
\text { coseas }\end{array}$ & 23 & 0.9969 & $4.5100-3$ & 0.9912 & 1.0075 & 3.94826 & $4.9292-3$ & 0.9884 & 0.9760 \\
\hline
\end{tabular}

The statistical treatment for generation of a linear uniform-width closed-interval tolerance band used in TM-238, was used to generate Figures C.1 through C.11. In each of these figures the calculated $k_{\text {eff }}$ is presented as a function of an independent variable; enrichment for Figure C.1 and AEG for the remaining figures. In each figure, the solid curve is a linear fit through the calculated data. The linear uniform width band that encompasses the lower 95\% confidence band on a single future calculation, and the $95 \%$ confidence uniform-width closed-interval lower tolerance band on $99.9 \%$ proportion of the population are also presented.

For these figures, the "bias" is defined as the difference between the linear least-squares fit through the data and a $k_{\text {eff }}=1.0$. The interpretation of the tolerance bands is similar to that for the grouped data. The linear uniform-width band that encompasses the $95 \%$ confidence band for a single future calculation may be interpreted as the $\mathrm{k}_{\text {eff }}$ value that, for a critical system similar to those validated, one would calculate above for a single future estimate of $\mathrm{k}_{\mathrm{eff}}$ at $95 \%$ confidence. Any calculated $\mathrm{k}_{\text {eff }}$ above this band is considered critical. The $95 \%$ closed-interval, uniform-width, lower tolerance band on $99.9 \%$ proportion of the population may be used as the calculational acceptance criteria. The values of $\mathrm{k}_{\text {eff }}$ represented by this band may be interpreted as the $\mathrm{k}_{\text {eff }}$ value that, for a critical system similar to those validated, one would expect to calculate above for $99.9 \%$ of the estimates of $k_{\text {eff }}$ at $95 \%$ confidence. Any calculated $k_{\text {eff }}$ below this band is considered to be of the group of systems that is not critical (For 
ease of discussion this band will be referred to as the subcritical band). The difference between the two confidence bands may be taken as the margin of calculated subcriticality. With the exception of Figure C.11, this margin is in excess of $0.02 \Delta \mathrm{k}$. The margin for Figure C.11 is $0.0147 \Delta \mathrm{k}$.

The significant effect of considering the sparsity of validation case for enrichments between 5 and $90 \%$ is shown in Figure C.1. The lack of data in this region causes the $95 \%$ confidence band for $99.9 \%$ of the population to be significantly lower than that estimated when treating the data as a group. The margin of calculated subcriticality for Figure $C .1$ is approximately $0.0488 \Delta \mathrm{k}$. This indicates that the code and cross sections should be used with caution when evaluating intermediate enrichment systems. Inclusion of critical experiments in this range would help to reduce the margin of calculated subcriticality while retaining the high degree of confidence in the prediction of subcritical systems.

A similar effect occurs when highly enriched validation cases are treated as a function of AEG over the full range of AEG as shown in Figures C.3 and C.8. Systems with an AEG in the intermediate range are uncommon. Separation of the experiments into low AEG and high AEG, as in Figures C.9 and C.10, eliminate some of the excess conservatism in the subcritical band in Figure C.8 with no reduction in the confidence of the prediction of subcritical systems.

The validation results indicate that KENO V.a and the 27 group library in NCSS accurately predict $k_{\text {eff }}$ over a broad range of critical experiments. In the evaluation of a fissile system, the analyst must determine if the range of the validation parameters is adequate. Figures C.1 through C.3 are considered most appropriate for the general case, but may be supplemented with Figures C.4 through C.11 and the group results presented in Table C.2. Analysis of the validation results indicates that intermediate enriched systems require a larger margin of subcriticality for a given confidence level due to the lack of critical experiments in this range.

The Table 5, high AEG results, presented in Figure C.10 indicate a calculational trend in $\mathrm{k}_{\mathrm{eff}}$ as a function of AEG. This trend is due to the bias in the validation experiment groups. The bias may be caused due to inadequate experimental descriptions or code and cross section bias. The use of Figure C. 3 for the subcritical band for highly enriched systems would account for this bias in any event. 


\section{$k$-effective vs enrichment \\ high and low enriched cases}

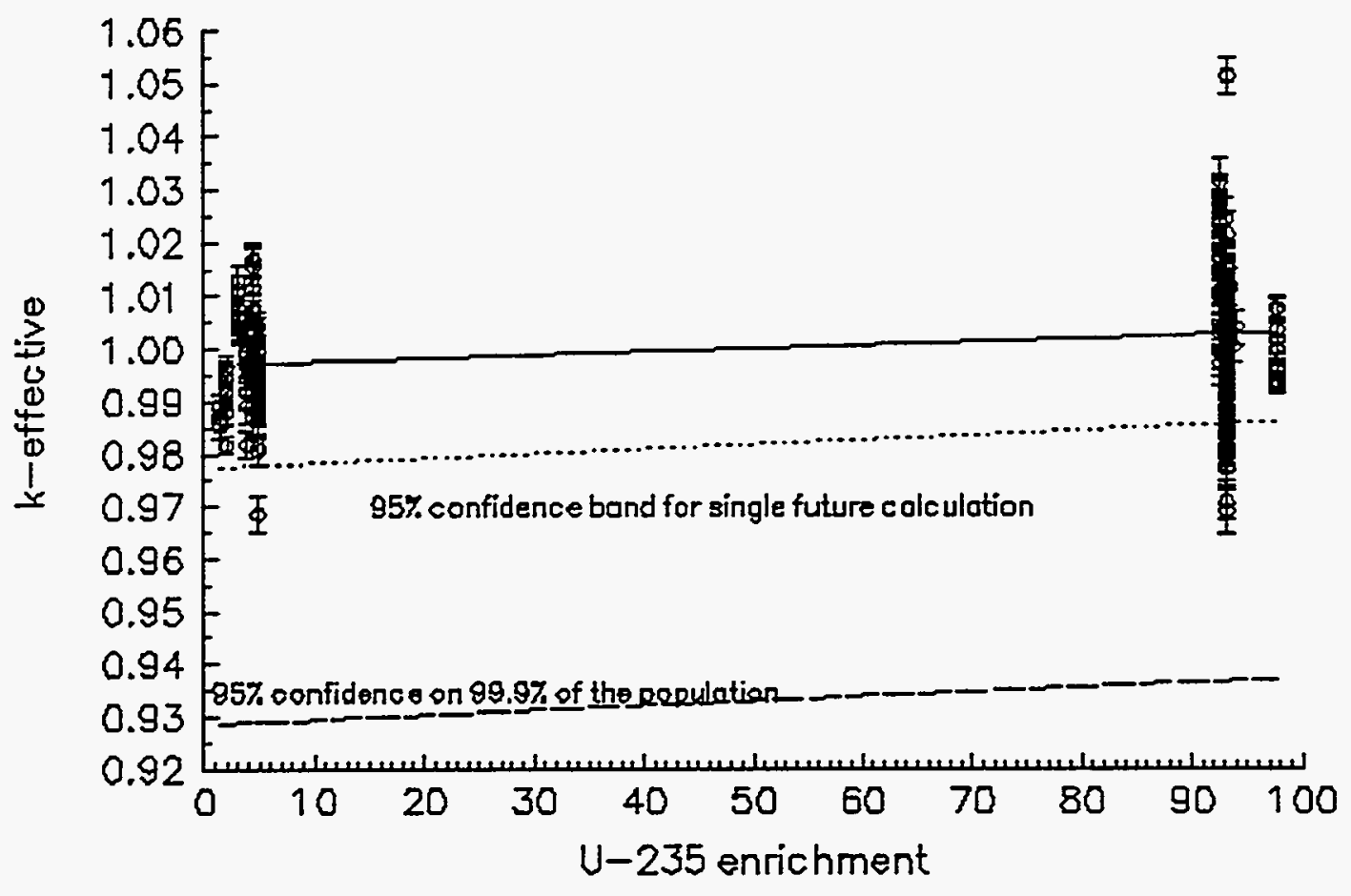

Figure C-1. $\mathrm{K}_{\mathrm{eff}}$ vs Enrichment - High and Low Enriched Cases

C-4 


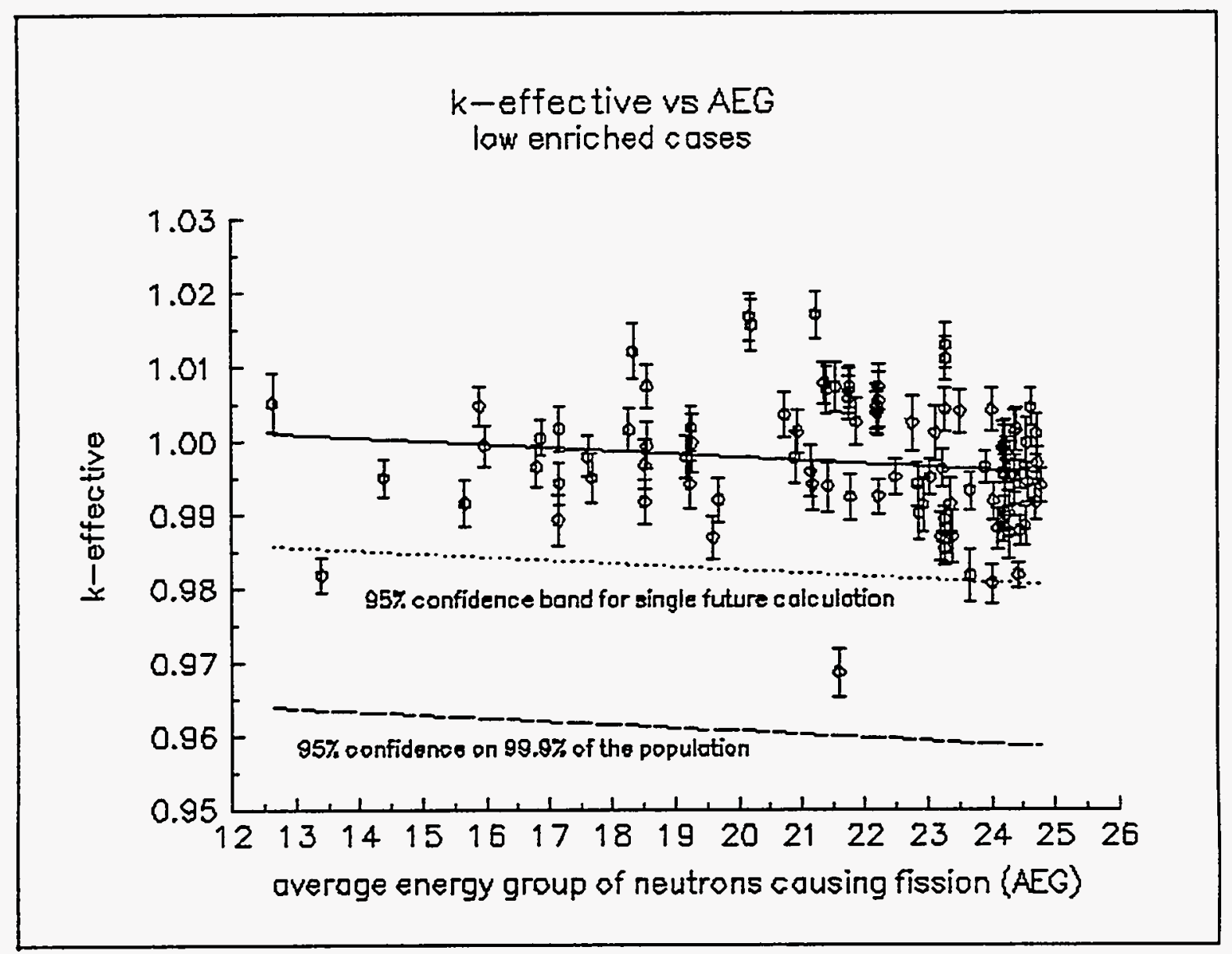

Figure C-2. $\mathrm{K}_{\text {eff }}$ vs AEG - Low Enriched Cases 
$k$-effective vs AEG

high enriched cases

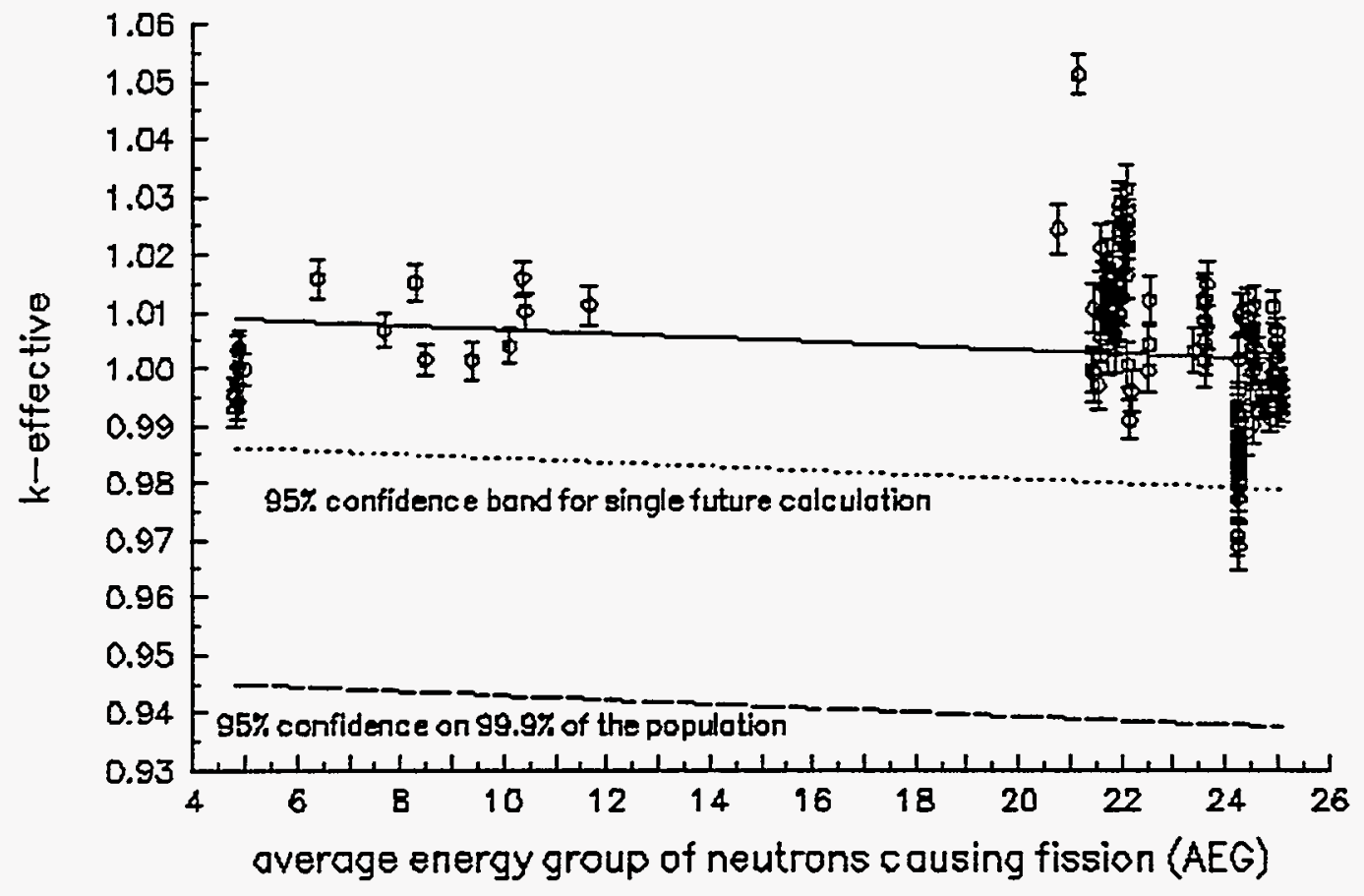

Figure C-3. $\mathrm{K}_{\mathrm{eff}}$ vs AEG - Highly Enriched Cases 
$k$-effective vs AEG

Table 1 cases

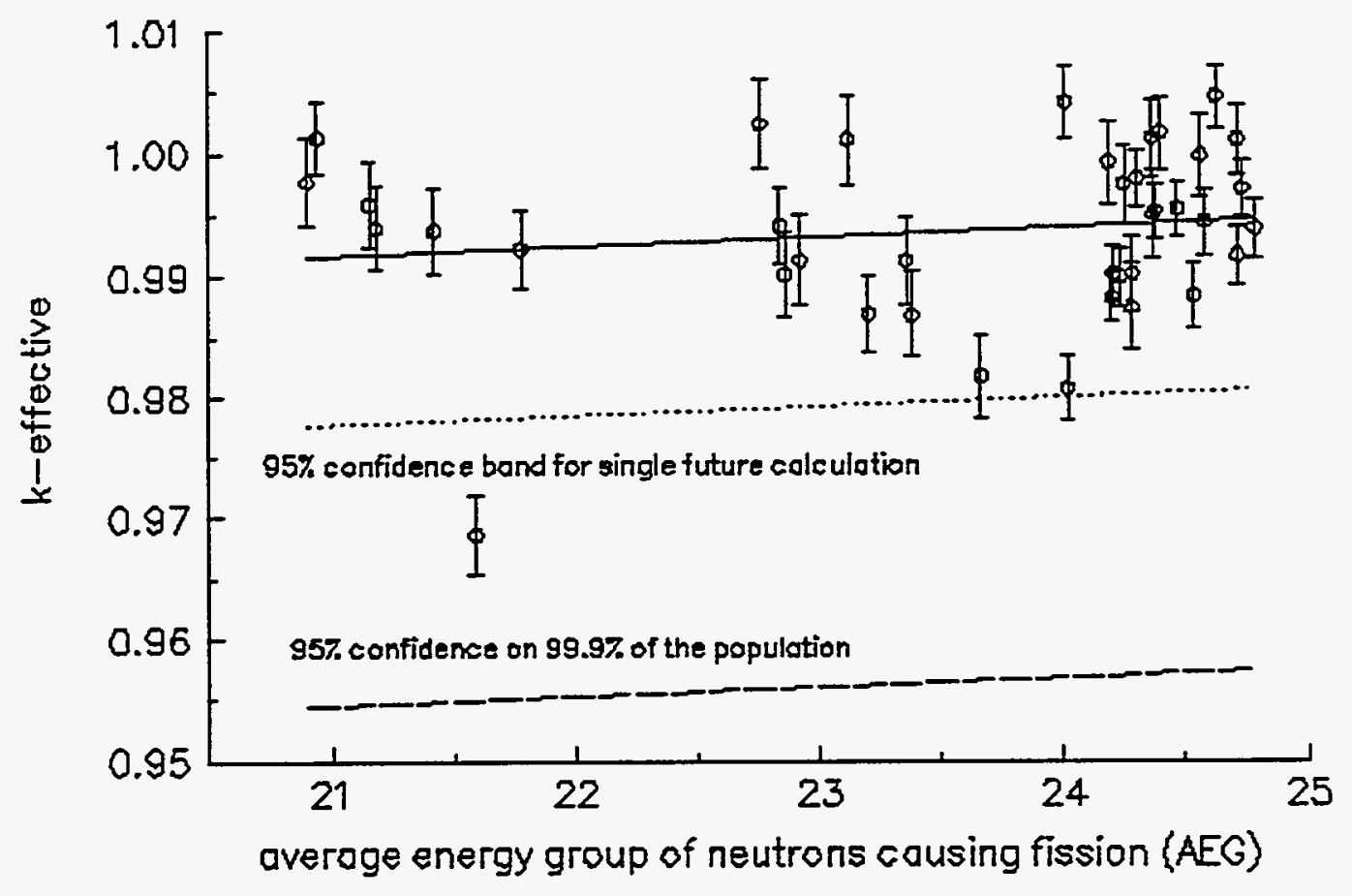

Figure C-4. $\mathrm{K}_{\mathrm{eff}}$ vs AEG - Table 1 Cases

C-7 


\section{$k$-effective vs AEG \\ Table 2 coses}

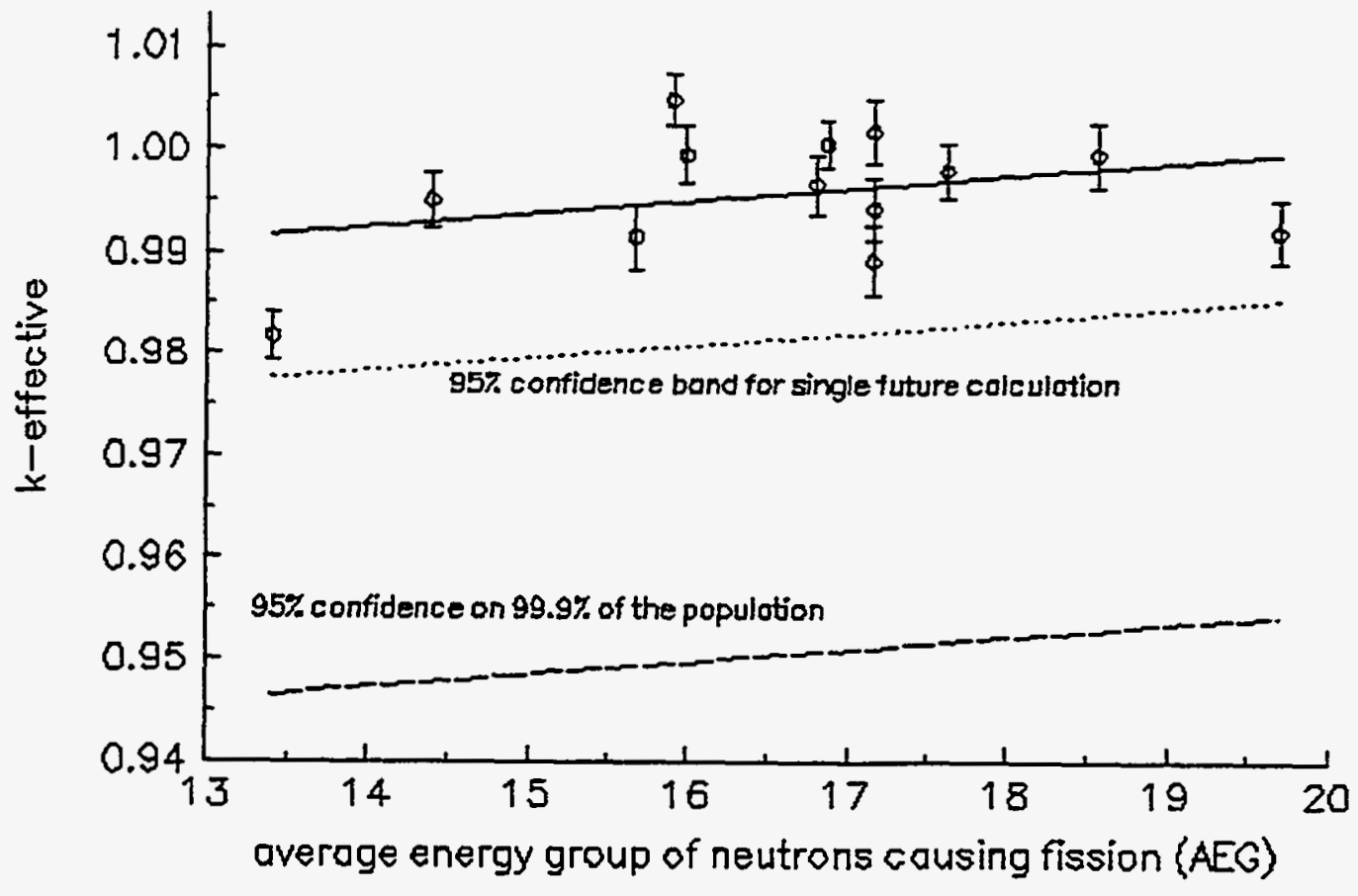

Figure C-5. $\mathrm{K}_{\mathrm{eff}}$ vs AEG - Table 2 Cases

C-8 


\section{$k$-effective vs AEG \\ Table 3 cases}

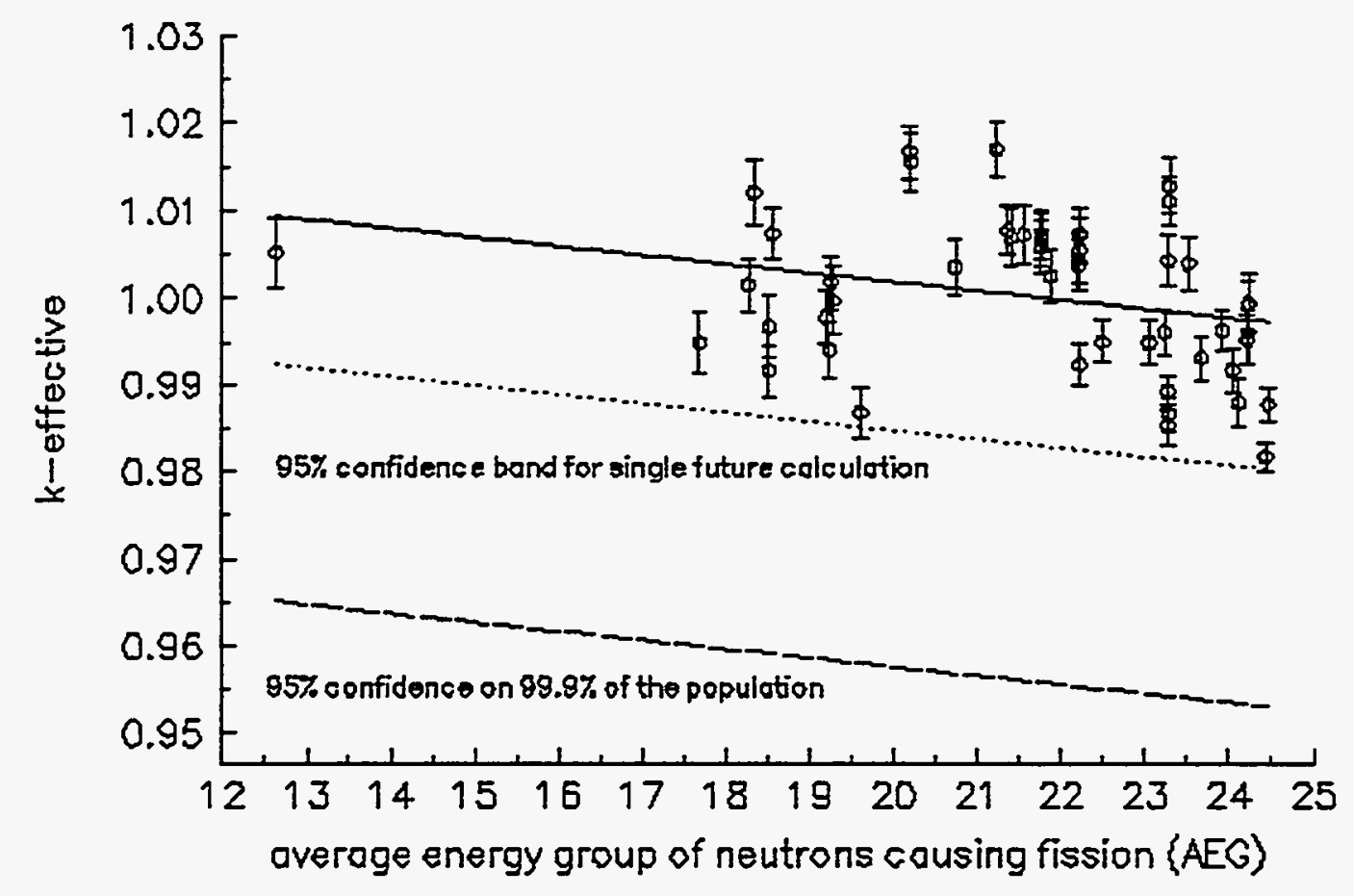

Figure C-6. $\mathrm{K}_{\text {eff }}$ vs AEG Table 3 Cases 


\section{$k$-effective vs AEG \\ Table 4 cases}

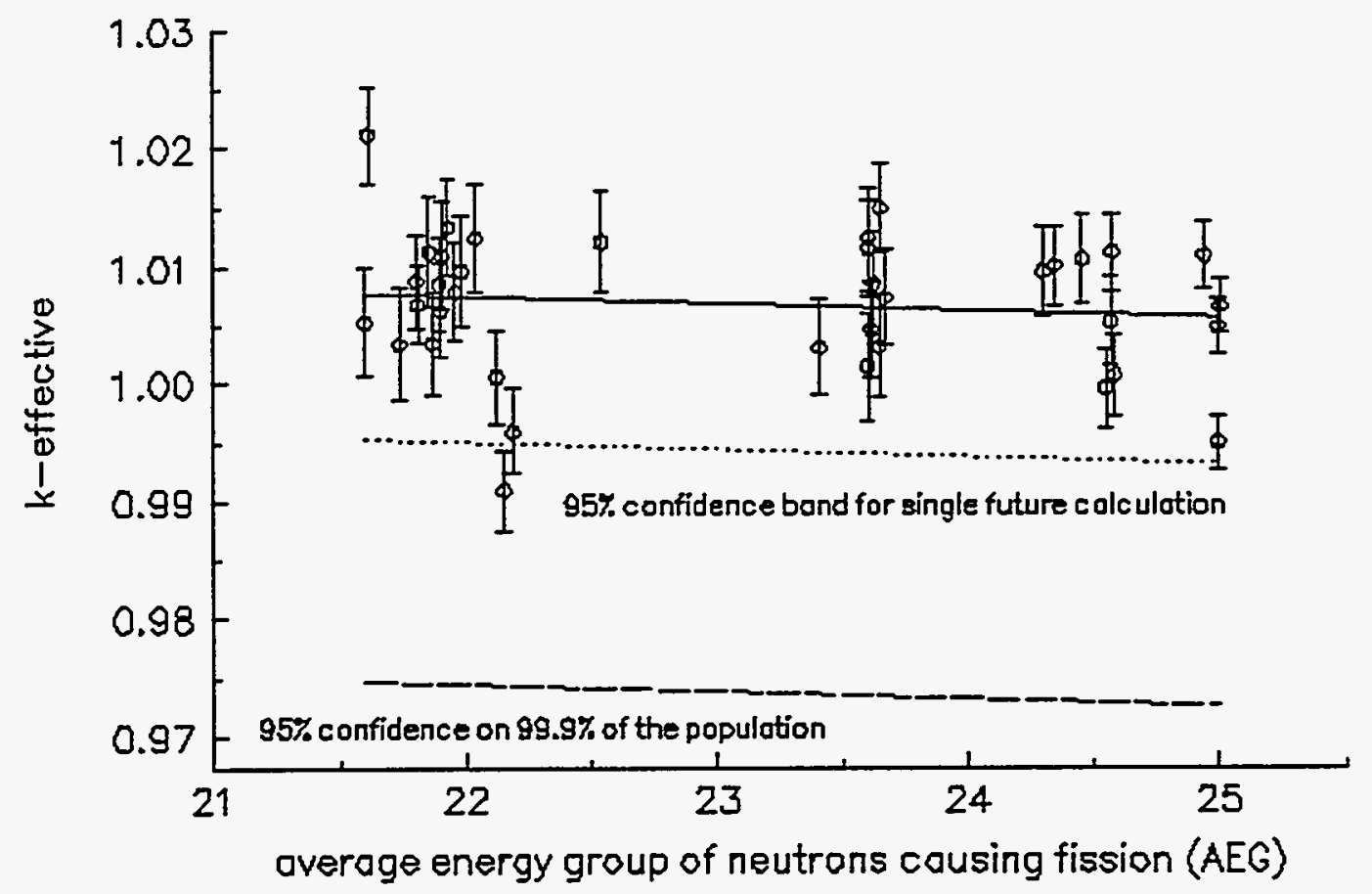

Figure C-7. $\mathrm{K}_{\text {eff }}$ vs AEG - Table 4 Cases 


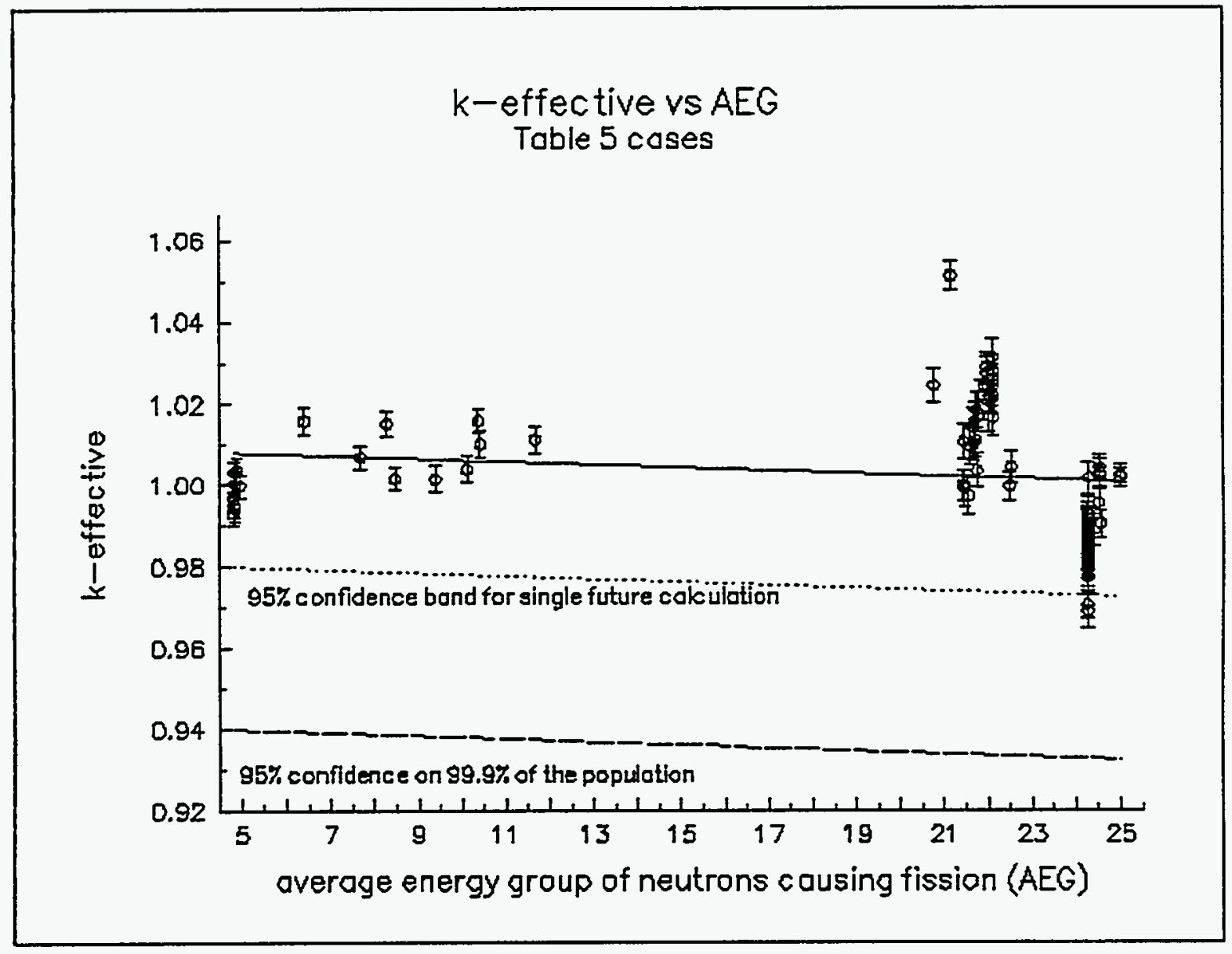

Figure C-8. $K_{\text {fff }}$ vs AEG - Table 5 Cases

C-11 


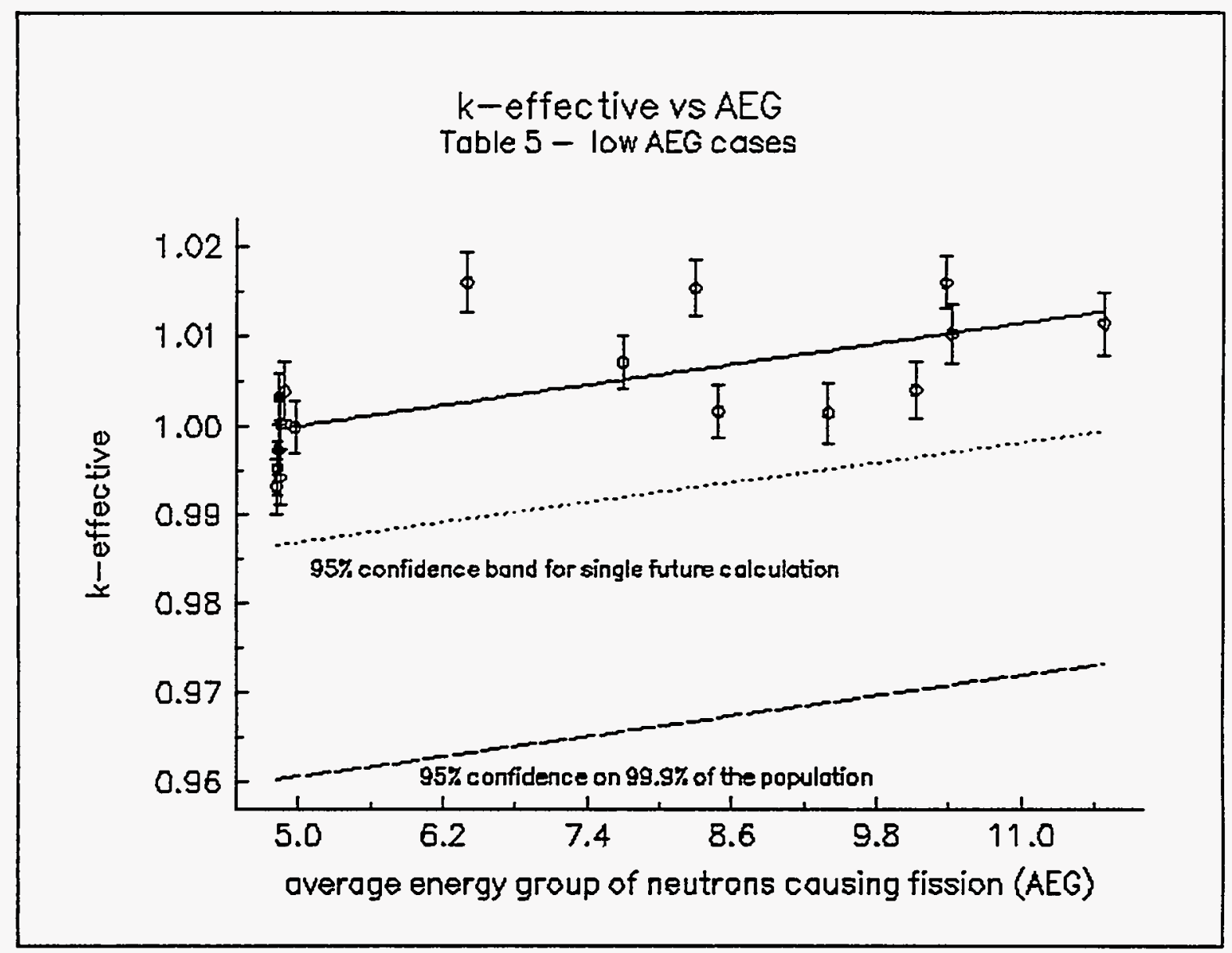

Figure C-9. $\mathrm{K}_{\text {eff }}$ vs AEG - Table 5 Low AEG Cases 
$k$-effective vs AEG

Table 5 - high AEG cases

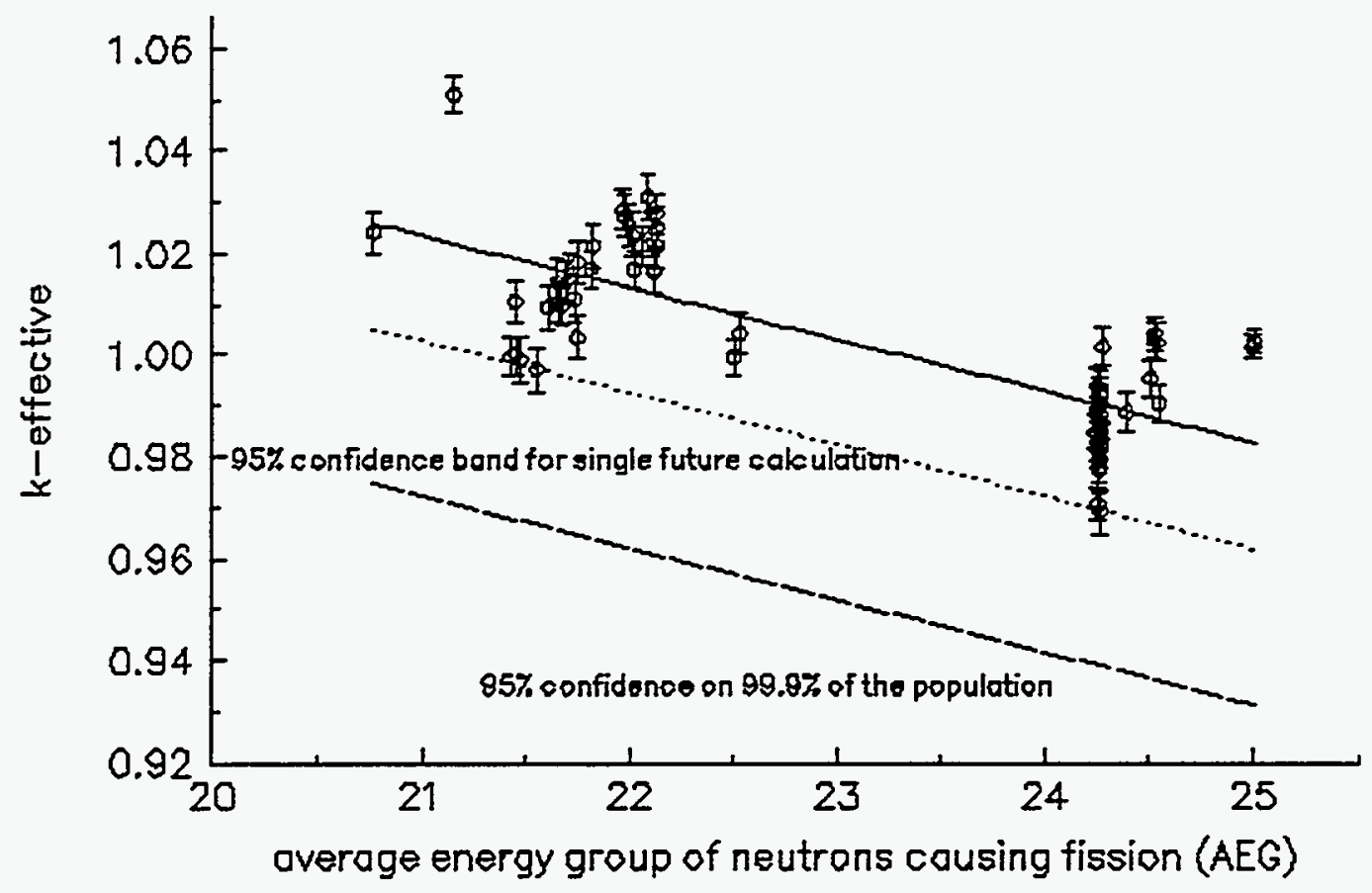

Figure 10. $\mathrm{K}_{\mathrm{eff}}$ vs AEG - Table 5 High AEG Cases

C-13 


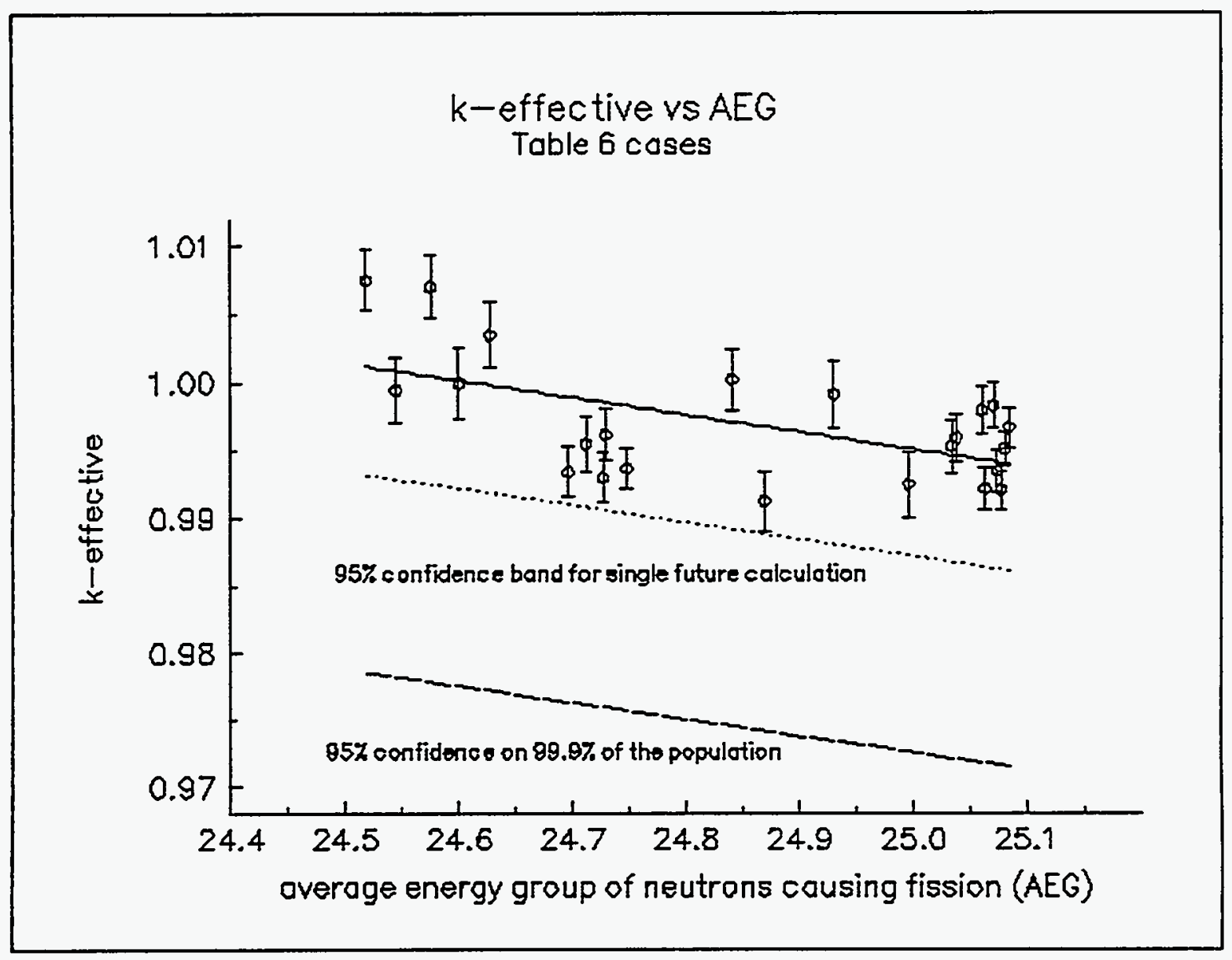

Figure $\mathrm{C}-11 . \mathrm{K}_{\text {eff }}$ vs AEG - Table 6 Cases

C-14 


\section{Appendix D: Calculated Results and Experimental Parameters}

In order to analyze the data from the validation calculations, the experiments were parameterized. The results could then be categorized in a number of ways to test for trends and bias. Table D.1 contains the parameters which were used for data analysis for each experiment.

Key terms are as follows:

\begin{tabular}{|c|c|c|}
\hline RUN & - & Sequential number assigned to experiments. \\
\hline MEMB & - & Case number which corresponds to Tables $1-6$. \\
\hline ENRLEV & - & Enrichment level (High, Intermediate, Low). \\
\hline AEG & - & Average Energy Group causing fission. \\
\hline ASSAY & - & Fuel enrichment. \\
\hline REFLCOND & - & $\begin{array}{l}\text { Reflection condition (Reflected, Partially reflected, } \\
\text { Unreflected). }\end{array}$ \\
\hline REFL & - & Reflector material. \\
\hline SYSGEOM & - & Overall system geometry (Array, Single unit, Mixed). \\
\hline UNITGEOM & - & Principal geometry of unit (CYlinder, SLab, SPhere). \\
\hline FUELMIX & & $\begin{array}{l}\text { Indicator as to whether the system was considered primarily } \\
\text { HETerogeneous or HOMogeneous. }\end{array}$ \\
\hline FUEL & - & Primary form of fissile material. \\
\hline FMATL & - & Principal fissile nuclide in fuel. \\
\hline REF & - & Reference number for reference $v$ \\
\hline
\end{tabular}


Tsble D. 1. Colculational resulta and experimentel paremetere

\begin{tabular}{|c|c|c|c|c|c|c|c|c|c|c|c|c|}
\hline RUN & MEMB & AEO & ENRLEV & ASSAY & REFLCOND & REFL $S$ & SYSOEOM & UN17 & FUELMIX & FUEL & FMATL & REF \\
\hline 1 & CAMO1 & 21.1822 & L & 4.88 & R & H2O & A & CY & HET & METAL & U235 & 14 \\
\hline 2 & CAMO2 & 21.7703 & $i$ & 4.80 & $\mathbf{R}$ & $\mathrm{H} 2 \mathrm{O}$ & A & cr & HET & METAL & U236 & 14 \\
\hline 3 & CMMO3 & 22.9244 & L & 4.88 & A & $\mathrm{H}_{2} \mathrm{O}$ & A & CY & HET & METAL & U236 & 14 \\
\hline 4 & CAAO4 & 23.1204 & $\mathbf{l}$ & 4.89 & A & $\mathrm{H} 2 \mathrm{O}$ & A & cr & HET & METAL & U236 & 14 \\
\hline 5 & CAMOE & 23.2062 & L & 4.89 & $\mathbf{R}$ & H2O & A & CY & HET & METAL & U235 & 14 \\
\hline - & CMOOS & 21.1620 & $\mathrm{~L}$ & 4.89 & A & СOMP & $A$ & cY & HET & METAL & U236 & 14 \\
\hline 7 & CAMO7 & 21.4201 & $i$ & 4.89 & $\mathbf{R}$ & PB & A & CY & HET & METAL & U236 & 14 \\
\hline - & CAMOB & 22.8396 & 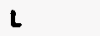 & 4.80 & A & PB & $A$ & cr & HET & METAL & U235 & 14 \\
\hline ? & CMAOS & 22.7681 & L & 4.80 & $\mathbf{R}$ & PB & A & $\mathrm{Cr}$ & HET & METAL & U236 & 14 \\
\hline 10 & CM1O & 20.0405 & L & 4.89 & A & PB & $A$ & CY & HET & METAL & U235 & 14 \\
\hline 11 & CMII & 20.8007 & $i$ & 4.80 & $\mathbf{R}$ & PB & A & cr & HET & METAL & U236 & 14 \\
\hline 12 & CM12 & 24.0220 & L & 4.89 & R & UO2F2 & $A$ & cr & HET & METAL & U236 & 14 \\
\hline 13 & CM13 & 24.3789 & $\mathbf{i}$ & 4.89 & $\mathbf{R}$ & UO2F2 & A & $\mathrm{CY}$ & HET & METAL & U236 & 14 \\
\hline 14 & CM14 & 24.4714 & $\mathrm{~L}$ & 4.89 & $\mathbf{R}$ & UO2F2 & A & cr & HET & METAL & U236 & 14 \\
\hline 16 & CAM16 & 24.2016 & L & 4.89 & A & UO2F2 & $A$ & cr & HET & METAL & U236 & 14 \\
\hline 10 & CMAIO & 24.2118 & $\mathbf{t}$ & 4.89 & A & UO2P2 & A & $c \gamma$ & HET & METAL & U236 & 14 \\
\hline 17 & CMA17 & 24.2302 & $i$ & 4.89 & A & UO2F2 & A & CY & HET & METAL & U236 & 14 \\
\hline 18 & CM18 & 24.3070 & $i$ & 4.80 & A & U02F2 & $A$ & cY & HET & METAL & U236 & 14 \\
\hline 10 & CAM10 & 23.3604 & $i$ & 4.80 & u & & s & SL & HOM & U308 & U236 & 16 \\
\hline 20 & CM120 & 24.0100 & L & 4.89 & u & & $\mathbf{s}$ & SL & HOM & U308 & U236 & 16 \\
\hline 21 & CMA21 & 24.6858 & L & 4.88 & u & & $\mathbf{s}$ & SL & ном & U308 & U236 & 15 \\
\hline 22 & CMA22 & 24.2627 & L & 4.80 & $u$ & & s & st & HOM & U308 & U235 & 18 \\
\hline 23 & CMA23 & 23.3810 & $i$ & 4.89 & A & $\mathrm{H} 2 \mathrm{O}$ & s & sL & HOM & U308 & U236 & 16 \\
\hline 24 & CMA24 & 23.6840 & L & 4.80 & A & H2O & s & st & HOM & U308 & U236 & 16 \\
\hline 25 & CAM26 & 24.1880 & L & 4.89 & A & $\mathrm{H} 2 \mathrm{O}$ & s & si & HOM & U308 & U236 & 16 \\
\hline 20 & CMA2O & 24.0282 & L & 4.89 & A & H2O & s & st & HOM & U308 & U236 & 16 \\
\hline 27 & CMA27 & 22.8612 & L & 4.89 & A & H2O & $s$ & st. & HOM & U308 & U236 & 16 \\
\hline 28 & CMA28 & 21.6801 & L & 4.80 & A & H2O & s & st & HOM & U3OB & U236 & 16 \\
\hline 20 & CMA20 & 24.3721 & L & 4.80 & A & H2O & 3 & St & HOM & U30B & U236 & 15 \\
\hline 30 & CMA30 & 24.2872 & $i$ & 4.80 & $u$ & & s & $c Y$ & HOM & UO2F2 & U236 & 18 \\
\hline 31 & CAA31 & 24.2824 & $i$ & 4.08 & $u$ & & $s$ & st & HOM & UO2F2 & U235 & 16 \\
\hline 32 & CMA32 & 24.6307 & $l$ & 1.80 & u & & s & cY & НОМ & UO2F2 & U235 & 16 \\
\hline 33 & CMA33 & 24.7105 & $i$ & 4.89 & u & & s & SP & HOM & UO2F2 & U236 & 16 \\
\hline 34 & CMA34 & 24.7177 & $\mathbf{i}$ & 4.80 & u & & s & cr & HOM & UO2F2 & U236 & 16 \\
\hline 36 & CMA35 & 24.3051 & L & 4.80 & ต & H 20 & s & $\mathrm{cr}$ & HOM & VO2F2 & U236 & 16 \\
\hline 36 & CAA3O & 24.4004 & $\mathbf{L}$ & 4.60 & A & H2O & s & SL & HOM & UO2F2 & U236 & 16 \\
\hline 37 & CMM37 & 24.6827 & L & 4.80 & A & H2O & 3 & $\mathrm{cr}$ & HOM & UO2F2 & U236 & 16 \\
\hline 38 & CMA3B & 24.7862 & L & 4.00 & A & $\mathrm{H} 2 \mathrm{O}$ & s & $\mathbf{S P}$ & HOM & UO2F2 & U235 & 16 \\
\hline 30 & CAM39 & 24.7339 & L & a.80 & A & H2O & s & cr & HOM & UO2F2 & U235 & 16 \\
\hline
\end{tabular}


Teble D.I (continued)

\begin{tabular}{|c|c|c|c|c|c|c|c|c|c|c|c|c|}
\hline RUN & MEMB & AEO & ENRLEV & ASSAY & REFLCOND & RER & SYSGEOM & UNITGEOM & FUELMIX & FUE. & FMATL & REF \\
\hline 40 & CABOI & 14.3919 & $\mathbf{L}$ & 3.86 & R & $\mathrm{H} 2 \mathrm{O}$ & A & CY & HET & METAL & U236 & 10 \\
\hline 11 & CABO2 & 16.0838 & l & 3.86 & A & $\mathrm{H} 2 \mathrm{O}$ & A & $\mathrm{Cr}$ & HET & METAL. & U235 & 16 \\
\hline 42 & CABO3 & 10.8027 & $L$ & 3.85 & A & $\mathrm{H} 2 \mathrm{O}$ & $A$ & cY & HET & METAL & U236 & 16 \\
\hline 43 & CABOT & 13.4020 & l & 3.85 & R & H2O & A & CY & HET & METAL & U235 & 16 \\
\hline 44 & CABOB & 16.6700 & L & 3.85 & $\mathbf{R}$ & $\mathrm{H} 2 \mathrm{O}$ & A & CY & HET & METAL & U235 & 16 \\
\hline 46 & CABOO & 17.1610 & L & 3.85 & $\mathbf{R}$ & $\mathrm{H} 2 \mathrm{O}$ & $A$ & cr & HET & METALL & U236 & 16 \\
\hline 40 & CAB10 & 10.8677 & L & 3.86 & A & $\mathrm{H} 2 \mathrm{O}$ & $A$ & CY & HET & METAL & U235 & 18 \\
\hline 47 & CAB11 & 17.1697 & $\mathbf{L}$ & 3.85 & $\mathbf{A}$ & $\mathrm{H} 2 \mathrm{O}$ & A & CY & HET & METAL. & U235 & 16 \\
\hline 48 & CAB12 & 17.0173 & $L$ & 3.86 & R & $\mathrm{H} 2 \mathrm{O}$ & A & cY & HET & METAL & U236 & 10 \\
\hline 40 & CAB 13 & 15.0018 & L & 3.86 & R & $\mathrm{H} 2 \mathrm{O}$ & A & cY & HET & METAL & U236 & 16 \\
\hline 60 & CAB14 & 17.1680 & i & 3.86 & $\mathbf{R}$ & $\mathrm{H} 2 \mathrm{O}$ & A & cY & HET & METAL & U236 & 10 \\
\hline 61 & CAB16 & 10.6672 & l & 3.86 & A & H2O & $A$ & cr & HET & METAL & U236 & 18 \\
\hline 62 & CABIO & 19.0900 & l & 3.86 & A & $\mathrm{H} 2 \mathrm{O}$ & A & cY & HET & METAL & U235 & 8 \\
\hline
\end{tabular}


Teblo D.1 (eontinued)

\begin{tabular}{|c|c|c|c|c|c|c|c|c|c|c|c|c|}
\hline RUN & MEMB & AEO & ENRLEV & ASSAY & REFLCOND & REFL & SYSOEOM & UNITGEOM & FUELMIX & FUer & FMATL & REF \\
\hline 63 & $\mathrm{CASO} 4$ & 23.2734 & $\mathbf{L}$ & 1.40 & $u$ & & s & st & HOM & UF4 & U235 & 17 \\
\hline 64 & CASOE & 23.2888 & $\mathbf{L}$ & 1.40 & u & & $\mathbf{s}$ & SL & HOM & UFA & U235 & 17 \\
\hline 66 & CASOO & 23.2791 & $L$ & 1.40 & u & & s & SL & HOM & UF4 & U235 & 17 \\
\hline 60 & CASII & 22.4862 & L & 2.00 & R & PARA & $\mathbf{s}$ & st. & ном & UF4 & U235 & 18 \\
\hline 67 & CAS12 & $22.218 \theta$ & $L$ & 2.00 & $u$ & & s & St & HOM & UFA & U235 & 18 \\
\hline 68 & CAS13 & 23.2281 & $L$ & 2.00 & A & PARA & s & st & HOM & UFA & U235 & 18 \\
\hline 69 & CAS14 & 23.0448 & $\mathbf{L}$ & 2.00 & u & & $\mathbf{s}$ & st. & ном & UFA & U235 & 18 \\
\hline 00 & CAS16 & 23.0689 & $i$ & 2.00 & R & PARA & s & St. & HOM & UFA & U235 & 18 \\
\hline 61 & CAS1O & 23.0141 & $\mathbf{L}$ & 2.00 & R & PARA & $\mathbf{s}$ & st. & HOM & UFA & U236 & 18 \\
\hline 02 & CASIT & 24.1084 & L & 2.00 & A & PARA & $\mathbf{s}$ & St & HOM & UFA & U236 & 18 \\
\hline 03 & CASIO & 24.0407 & L & 2.00 & u & & s & st. & HOM & UFA & U236 & 18 \\
\hline 04 & CAS1E & 24.4662 & L & 2.00 & A & PARA & s & st & HOM & UFA & U235 & 18 \\
\hline 06 & CAS2O & 24.4267 & $\mathbf{L}$ & 2.00 & $u$ & & s & st & HOM & UFA & U235 & 18 \\
\hline 68 & CAS21 & 22.1886 & L & 3.00 & R & PARA & $\mathbf{s}$ & st & HOM & UFA & U235 & 18 \\
\hline 07 & CAS22 & 22.1997 & $\mathbf{L}$ & 3.00 & A & PARA & s & SL. & ном & UFA & U235 & 18 \\
\hline 68 & CAS23 & 22.2168 & L & 3.00 & R & PARA & s & St. & HOM & UFA & U235 & 18 \\
\hline 60 & CAS24 & 22.2180 & l & 3.00 & R & PARA & $\mathbf{s}$ & SL & HOM & UFA & U235 & 18 \\
\hline 70 & CAS25 & 22.2128 & L & 3.00 & $\mathbf{R}$ & PARA & $\mathbf{s}$ & st & HOM & UF4 & U235 & 18 \\
\hline 71 & CAS20 & 21.7608 & $\mathrm{~L}$ & 3.00 & $u$ & & s & St. & HOM & UFA & U235 & 18 \\
\hline 72 & CAS27 & 21.7633 & i & 3.00 & $u$ & & s & st & HOM & UFA & U235 & 10 \\
\hline 73 & CAS28 & 21.7633 & $\mathbf{L}$ & 3.00 & $u$ & & $\mathbf{s}$ & st & HOM & UFA & U235 & 18 \\
\hline 74 & CAS20 & 23.6114 & $\mathbf{L}$ & 3.00 & R & POLY & $\mathbf{s}$ & st. & HOM & UF4 & U236 & 18 \\
\hline 76 & CAs3o & 23.2814 & $i$ & 3.00 & u & & s & st & HOM & UFA & U235 & 18 \\
\hline 70 & CASBI & 23.2732 & $i$ & 3.00 & u & & $\mathbf{s}$ & st & ROM & UFA & U235 & 18 \\
\hline 77 & CAS32 & 23.2821 & $L$ & 3.00 & u & & $\mathbf{s}$ & st & HOM & UFA & U236 & 18 \\
\hline 78 & CAS33 & 24.2140 & L & 4.98 & ค & COMP & s & cr & HOM & UO2F2 & U236 & 10 \\
\hline 70 & CAS34 & 24.2271 & L & 4.98 & R & сомP & s & cr & HOM & UO2F2 & U236 & 18 \\
\hline 80 & CAS3E & 24.2367 & L. & 4.08 & u & & $\mathbf{s}$ & SP & HOM & UO2F2 & U236 & 20 \\
\hline 01 & CAS3O & 24.2270 & $\mathbf{L}$ & 4.08 & u & & s & CY & HOM & UO2P2 & U236 & 21 \\
\hline 82 & CARO1 & 21.3614 & $\mathrm{i}$ & 4.40 & A & PLEX & $\mathbf{s}$ & st. & HET & U308 & U236 & 22 \\
\hline 83 & CARO2 & 21.3807 & $i$ & 4.46 & A & PLEX & $s$ & St & HET & U308 & U235 & 22 \\
\hline 84 & CARO3 & 10.0039 & i & 4.46 & $\mathbf{R}$ & PLEX & $\mathbf{s}$ & SL & HET & U308 & U236 & 22 \\
\hline 86 & CARO4 & 21.2247 & L & 4.40 & A & CONC & $\mathrm{s}$ & SL & HET & U30B & U236 & 22 \\
\hline 86 & CAROE & 10.1879 & L & 4.46 & R & CONC & $\mathbf{s}$ & st & HET & U30B & U235 & 22 \\
\hline 87 & CAROO & 12.0293 & i & 4.40 & R & PLEX & $s$ & st & HOM & U308 & U236 & 23 \\
\hline 80 & CAR07 & 18.3286 & L & 4.40 & A & PLEX & s & st & HOM & U300 & U235 & 23 \\
\hline 89 & CAROB & 10.2732 & l & 4.40 & A & PLEX & $\mathbf{s}$ & st. & HOM & U308 & U235 & 23 \\
\hline 80 & CAROO & 19.2330 & $i$ & 4.40 & A & PLEX & $\mathbf{s}$ & st. & HOM & U308 & U235 & 23 \\
\hline 01 & CAR1O & 17.0770 & $i$ & 9.46 & A & CONC & 8 & st & HOM & U308 & U235 & 23 \\
\hline 92 & CARII & 28.6300 & l & 4.46 & A & PLEXX & s & st & HET & U308 & U235 & 24 \\
\hline 93 & CAR12 & 20.1841 & $\mathrm{~L}$ & 4.40 & A & PLEX & $\mathbf{s}$ & st. & HET & U308 & U236 & 24 \\
\hline 94 & CAR13 & 20.2041 & $\mathbf{L}$ & 4.40 & A & PLEX & s & st & HET & U308 & U236 & 24 \\
\hline 96 & CAR14 & 18.6538 & $\mathrm{l}$ & 4.46 & A & PLEX & $\mathbf{s}$ & st. & HOM & U308 & U236 & 24 \\
\hline 90 & CAR15 & 18.6160 & L & 4.40 & A & PLEX & $\mathbf{s}$ & SL & HOM & Uзов & U236 & 24 \\
\hline 97 & CAR1O & 19.2531 & $L$ & 4.40 & A & PLEX & $\mathbf{s}$ & st. & HOM & U308 & U236 & 24 \\
\hline 98 & CARIT & $20.736 \theta$ & l & 4.40 & A & PLEX & $\mathbf{s}$ & st. & НЕT & U308 & U235 & 26 \\
\hline 89 & CAR18 & 21.8670 & L & 4.40 & A & PLEX & $\mathbf{s}$ & SL & HET & U308 & U236 & 25 \\
\hline 100 & CAR1O & 10.2868 & $i$ & 4.40 & A & PLEX & $\mathbf{s}$ & st & ном & U308 & U235 & 25 \\
\hline 101 & CAR2O & 18.6148 & L & 4.48 & A & PLEX & $\mathbf{s}$ & st & MOM & U308 & บ236 & 25 \\
\hline
\end{tabular}


Teblo D. 1 (eontinued)

\begin{tabular}{|c|c|c|c|c|c|c|c|c|c|c|c|c|}
\hline RUN & MEMB & AEO & ENRLEV & ASSAY & REFLCOND & RER & SYSGEOM & UNITGEOM & FUELMIX & FUEL & FMATL & REF \\
\hline 102 & CAMOI & 24.0397 & H & 03.20 & $u$ & & $\mathbf{s}$ & SP & ном & U02F2 & 0235 & 28 \\
\hline 103 & CAMO2 & 25.0034 & H & 93.20 & u & & $\mathbf{s}$ & SP & HOM & UO2F2 & U235 & 20 \\
\hline 104 & CMO3 & 24.09084 & H & 93.20 & $\mathbf{u}$ & & $\mathbf{s}$ & SP & HOM & NITRATE & U235 & 20 \\
\hline 105 & CMOA & 22.5400 & H & 93.20 & A & $\mathrm{H} 2 \mathrm{O}$ & $\mathbf{s}$ & SP & HOM & UO2F2 & U235 & 28 \\
\hline 108 & CMOS & 23.4107 & H & 93,20 & A & $\mathrm{H} 2 \mathrm{O}$ & s & SP & HOM & UO2F2 & U235 & 28 \\
\hline 107 & CMAOB & 24.0070 & H & 83.20 & A & $\mathrm{H} 2 \mathrm{O}$ & $\mathbf{s}$ & SP & HOM & UO2F2 & U236 & 28 \\
\hline$\cdot 108$ & CAAO7 & & H & 93.20 & A & $\mathrm{H} 2 \mathrm{O}$ & $\mathbf{s}$ & SP & HOM & UO2F2 & U236 & 27 \\
\hline$\cdot 109$ & CMOB & & H & 03.20 & A & H2O & $\mathbf{s}$ & SP & ном & UO2F2 & U236 & 27 \\
\hline$\cdot 110$ & CAMOO & & H & 93.20 & u & & s & SP & HOM & UO2F2 & U235 & 27 \\
\hline$\bullet 111$ & CAM1O & & H & 83.20 & A & H2O & $\mathbf{s}$ & SP & HOM & UO2F2 & U236 & 27 \\
\hline$\cdot 112$ & CMA11 & & H & 83.20 & A & H2O & $\mathbf{s}$ & SP & HOM & UO2F2 & U236 & 27 \\
\hline 113 & CM12 & 23.0024 & H & 93.20 & u & & $\mathbf{s}$ & $\mathrm{Cr}$ & HOM & NITRATE & U236 & 28 \\
\hline 114 & CM13 & 21.0104 & H & 93.20 & u & & $\mathbf{s}$ & cr & HOM & NITRATE & U236 & 28 \\
\hline 115 & CMIA & 24.6760 & H & 03.20 & u & & $\mathbf{s}$ & $\mathrm{cr}$ & HOM & NITRATE & U236 & 28 \\
\hline 110 & CM16 & 23.0600 & H & 03.20 & u & & $\mathbf{s}$ & $\mathrm{Cr}$ & HOM & NITRATE & U236 & 28 \\
\hline 117 & CMIS & 21.6981 & H & 93.20 & u & & $\mathbf{s}$ & $\mathrm{Cr}$ & HOM & NITAATE & U235 & $2 B$ \\
\hline 118 & CMA17 & 23.0266 & H & 93.20 & $\mathbf{R}$ & CONC & $\mathbf{s}$ & $\mathrm{cr}$ & HOM & NITAATE & U236 & 28 \\
\hline 110 & CAA18 & 21.8647 & H & 93.20 & A & CONC & s & $\mathrm{cr}$ & HOM & NITRATE & U235 & 28 \\
\hline 120 & CM19 & 23.6105 & H & 03.20 & A & CONC & $\mathbf{s}$ & cr & HOM & NITRATE & U236 & 28 \\
\hline 121 & CM2O & 21.8671 & H & 93.20 & R & CONC & $\mathbf{s}$ & $\mathrm{Cr}$ & HOM & NITRATE & U236 & 28 \\
\hline 122 & CAM21 & 23.6518 & H & 93.20 & R & CONC & $\mathbf{s}$ & $\mathrm{cr}$ & HOM & NITAATE & U236 & 28 \\
\hline 123 & CM22 & 21.2814 & H & 83.20 & R & CONC & $\mathbf{s}$ & $\mathrm{cr}$ & HOM & NITRATE & U235 & 28 \\
\hline 124 & CM23 & 23.6074 & H & 93,20 & R & PLEX & $s$ & $\mathrm{cr}$ & HOM & NITRATE & U235 & 28 \\
\hline 126 & CM24 & 21.8010 & H & 93.20 & $\mathbf{R}$ & PLEX & $\mathbf{s}$ & $\mathrm{cr}$ & HOM & NITRATE & v235 & 28 \\
\hline 128 & CMA25 & 23.0035 & H & 93.20 & A & PLEX & $\mathbf{s}$ & $\mathrm{Cr}$ & HOM & NITRATE & U236 & 28 \\
\hline 127 & CAM26 & 21.8090 & H & 93.20 & $\mathbf{R}$ & PLEX & 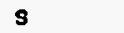 & $\mathrm{cr}$ & HOM & NITRATE & U236 & 28 \\
\hline 128 & CM27 & 23.0700 & H & 93.20 & R & PLEX & s & CY & HOM & NITRATE & U235 & 28 \\
\hline 120 & CMA2B & 22.03BB & H & 93.20 & A & PLEX & $s$ & $\mathrm{Cr}$ & HOM & NITRATE & U235 & 28 \\
\hline 130 & CM2O & 24.4663 & H & 93.20 & A & CONC & A & $\mathrm{cr}$ & HOM & NITRATE & U236 & 28 \\
\hline 131 & CAM30 & 21.0201 & H & 93.20 & A & CONC & A & $c r$ & Hом & NITRATE & U236 & 28 \\
\hline 132 & CMA31 & 24.2093 & H & 03.20 & A & CONC & A & $\mathrm{Cr}$ & ном & NITRATE & U235 & 28 \\
\hline 133 & CAM32 & 21.0565 & $H$ & 03.20 & A & CONC & A & cr & HOM & NITRATE & U236 & 28 \\
\hline 134 & CAM33 & 24.3476 & H & 03.20 & A & CONC & A & $\mathrm{Cr}$ & HOM & NITRATE & U236 & 28 \\
\hline 136 & CAM34 & 21.7360 & $H$ & 93.20 & A & CONC & $A$ & cr & ном & NITRATE & U236 & 28 \\
\hline 130 & CAM35 & 21.8980 & H & 03.20 & ค & CONC & A & cr & HOM & NITRATE & U236 & 28 \\
\hline 137 & CAM30 & 21.8042 & $H$ & 03.20 & A & CONC & A & $\mathrm{cr}$ & ном & NITRATE & U236 & 28 \\
\hline 138 & CAM37 & 24.6688 & H & 93.20 & A & PLEX & A & $\mathrm{cr}$ & HOM & NITRATE & U236 & 28 \\
\hline 139 & CM338 & 22.1633 & H & 03.20 & A & PLEX & $A$ & $\mathrm{cr}$ & Hом & NITRATE & U236 & 28 \\
\hline 140 & CMA30 & 24.6841 & $H$ & 03.20 & A & PLEX & A & cr & HOM & NITAATE & U235 & 28 \\
\hline 141 & CMAO & 22.1872 & H & 03.20 & A & PLEX & A & Cr & HOM & NITAATE & U236 & 28 \\
\hline 142 & CAMA1 & 24.6604 & H & 93.20 & A & PLEX & A & cr & HOM & NITRATE & U236 & 28 \\
\hline 143 & CMA2 & 21.8938 & H & 93.20 & A & PLEX & A & $\mathrm{cr}$ & HOM & NITRATE & U236 & 28 \\
\hline 144 & CAMA3 & 22.1210 & H & 93.20 & A & PLEX & $A$ & cr & HOM & NITRATE & U235 & 28 \\
\hline
\end{tabular}


Teble D.1 (contlinued)

\begin{tabular}{|c|c|c|c|c|c|c|c|c|c|c|c|c|}
\hline RUN & MEMB & AEO & ENRLEV & ASSAY & AEFLCOND & REFL & SYSGEOM & UNITGEOM & FUELMIX & FUE. & FMATL & REF \\
\hline 145 & CASOI & 4.8602 & H & 03.80 & $u$ & & $\mathbf{s}$ & $\mathbf{S P}$ & HOM & METAL & U235 & 20 \\
\hline 140 & $\mathrm{CASO2}$ & 0.4884 & H & 07.67 & A & $\mathrm{H} 2 \mathrm{O}$ & $\mathbf{s}$ & SP & ном & METAL & U236 & 35 \\
\hline 147 & CASO3 & 21.7099 & H & 03.20 & u & & $\mathbf{s}$ & cr & ном & NITRATE & U235 & 28 \\
\hline 148 & CASOA & 4.8710 & H & 93.20 & u & & $\mathbf{s}$ & cr & ном & ALLOY & U235 & 30 \\
\hline 149 & CASOS & 25.0067 & H & 03.20 & u & & s & SP & HOM & VO2F2 & U236 & 20 \\
\hline 160 & CASOB & 24.9807 & H & 93.20 & u & & $\mathbf{s}$ & SP & HOM & NITRATE & U236 & 31 \\
\hline 161 & CASOZ & 11.0794 & H & $\$ 3.50$ & R & $\mathrm{H} 2 \mathrm{O}$ & $\mathbf{s}$ & $\mathbf{S P}$ & HET & METAL & U235 & 32 \\
\hline 152 & CASOB & 0.4040 & H & 83.20 & F & c & $\mathbf{s}$ & cr & HET & METAL & U235 & 33 \\
\hline 163 & CASOO & 4.8874 & H & 04.00 & h & U238 & $\mathbf{s}$ & SL & ном & METAL & U236 & 32 \\
\hline 164 & CASIO & 10.4190 & H & 93.10 & R & OIL & $\mathbf{s}$ & $\mathbf{S P}$ & HET & METAL & U235 & 33 \\
\hline 165 & CAs11 & 9.3081 & $\mathbf{H}$ & 93.10 & A & OIL & $\mathbf{s}$ & SP & HET & METAL & U236 & 34 \\
\hline 160 & CAs12 & 4.8288 & H & 03.20 & u & & $M$ & $\mathrm{cr}$ & HOM & METAL & U236 & 36 \\
\hline •167 & CAS13 & & H & 92.00 & $u$ & & A & cr & ном & NITRATE & U235 & 38 \\
\hline 168 & CAS14 & 21.1646 & $H$ & 93.20 & ค & PLEX & A & $\operatorname{MiX}$ & HOM & NITRATE & U235 & 30 \\
\hline 169 & CAS15 & 8.2044 & H & 93.20 & R & POLY & A & cr & HET & METAL & U235 & 40 \\
\hline$\cdot 160$ & CAS1O & & H & 02.00 & u & & A & cr & HOM & NITAATE & U235 & 38 \\
\hline - 109 & CAS17 & & H & 02.00 & R & PARA & A & cr & HOM & NITAATE & U236 & 38 \\
\hline$\cdot 102$ & CAS1B & & H & 92.00 & R & PLEX & A & $\mathrm{Cr}$ & Hом & NITRATE & U236 & 38 \\
\hline 103 & CAS18 & 20.7882 & H & 93.10 & $u$ & & A & CY & HOM & NItRATE & U235 & 41 \\
\hline$\cdot 104$ & CAS20 & & H & 93.20 & R & PLEX & A & CY & HOM & NITRATE & U235 & 28 \\
\hline$\cdot 188$ & CAS21 & & H & 03.20 & $\mathbf{R}$ & CONC & A & $\mathrm{cr}$ & HOM & NITAATE & U235 & 28 \\
\hline 100 & CAS22 & 4.8443 & H & 93.20 & u & & $\mathbf{M}$ & $\mathrm{CY}$ & ном & METAL & U236 & 30 \\
\hline 107 & CAS23 & 4.8334 & $H$ & 03.20 & $u$ & & $M$ & MIX & HOM & METAL & U236 & 36 \\
\hline 108 & CAs24 & 4,0200 & H & 03.20 & u & & A & CY & HOM & METAL. & U236 & 37 \\
\hline 168 & CAS26 & 4.8488 & H & 93.20 & u & & A & $\mathrm{Cr}$ & HOM & METAL & U236 & 37 \\
\hline 170 & CAS20 & 10.1240 & H & 93.20 & R & PARA & A & cr & HOM & METAL & U236 & 37 \\
\hline 171 & CAS27 & 10.3737 & H & 93.20 & R & PARA & $A$ & $\mathrm{cr}$ & HOM & METAL & U235 & 37 \\
\hline 172 & CAS28 & 7.6833 & $H$ & 83.20 & u & & A & $\mathrm{Cr}$ & HET & METAL & U236 & 37 \\
\hline •173 & CAS29 & & H & 92.00 & u & & $A$ & $\mathrm{Cr}$ & HOM & NITAATE & U236 & 38 \\
\hline 174 & CAS3O & 24.2771 & H & 03.20 & u & & $\mathbf{s}$ & sL. & HOM & UO2F2 & U235 & 13 \\
\hline 176 & CAS31 & 24.3917 & H & 03.20 & R & H2O & $\mathbf{s}$ & st & HOM & UO2F2 & U235 & 43 \\
\hline 170 & CAS32 & 24.2617 & H & 93.20 & $u$ & & $A$ & sL & HOM & U02F2 & U235 & 13 \\
\hline 177 & CAS33 & 24.6081 & H & 93.20 & A & $\mathrm{H} 2 \mathrm{O}$ & A & st & HOM & U02F2 & U236 & 43 \\
\hline 178 & CAS34 & 24.2631 & $H$ & 93.20 & $u$ & & A & St & HOM & U02F2 & U235 & 13 \\
\hline 178 & CAS3E & 24.6460 & H & 93.20 & R & H2O & A & SL & HOM & UO2F2 & U235 & 43 \\
\hline 180 & CAS3O & 24.2482 & $H$ & 93.20 & u & & A & st. & HOM & U02F2 & U235 & 43 \\
\hline 181 & CAS37 & 24.5318 & H & 93.20 & A & $\mathrm{H} 2 \mathrm{O}$ & A & st. & HOM & U02F2 & U235 & 43 \\
\hline 182 & CAS3B & 24.2404 & H & 93.20 & $u$ & & A & st & HOM & UO2F2 & U235 & 43 \\
\hline 103 & CAS39 & 24.6230 & H & 03.20 & R & H2O & A & st. & Hом & UO2F2 & U235 & 43 \\
\hline 184 & CASAO & 24.2443 & H & 93.20 & $u$ & & A & St. & HOM & VO2F2 & U236 & 13 \\
\hline 185 & CASAI & 24.2673 & H & 03.20 & $u$ & & A & st. & HOM & V02F2 & U235 & 43 \\
\hline 180 & CASA2 & 24.2063 & H & 03.20 & $u$ & & $A$ & st & HOM & U02F2 & U236 & 43 \\
\hline 187 & CASA3 & 24.2800 & H & 03.20 & $u$ & & A & st. & HOM & U02F2 & U236 & 13 \\
\hline 180 & CASA4 & 24.2692 & H & 03.20 & u & & $A$ & st. & HOM & U02F2 & U235 & 43 \\
\hline 189 & CASA6 & 24.2671 & H & 93.20 & $u$ & & $\mathbf{s}$ & st & ном & U02F2 & U236 & 43 \\
\hline 180 & CASAO & 24.2680 & H & 03.20 & $u$ & & A & st & HOM & U02F2 & U235 & 43 \\
\hline 101 & CASA7 & 24.2513 & H & 03.20 & $u$ & & $A$ & st & HOM & U02F2 & U235 & 43 \\
\hline 192 & CASAB & 24.2661 & H & 93.20 & $u$ & & A & St. & HOM & UO2F2 & U236 & 43 \\
\hline 103 & CASAO & 24.2577 & H & 03.20 & u & & $A$ & St & HOM & VO2F2 & U236 & 43 \\
\hline 184 & CASEO & 24.2668 & H & 93.20 & $u$ & & A & st & HOM & U02F2 & U235 & 13 \\
\hline
\end{tabular}


Teble D.1 (contlinued)

\begin{tabular}{|c|c|c|c|c|c|c|c|c|c|c|c|}
\hline RUN & MEMB & AEO & ENRLEV & ASSAY & AEFLCOND & AEFL & SYSOEOM & UNITCEOM & FUELMIX & FUEL & FMATL \\
\hline 188 & CASE4 & 24.2848 & H & 03.20 & $u$ & & A & st. & ном & U02F2 & U235 \\
\hline 190 & CASEE & 24.2884 & H & 93.20 & $u$ & & A & SL & HOM & U02F2 & U236 \\
\hline 200 & CASEO & 24.2868 & H & 03.20 & $u$ & & A & st & HOM & UO2F2 & U235 \\
\hline 201 & CASE7 & 24.2080 & H & 93.20 & u & & A & SL & HOM & UO2F2 & U236 \\
\hline 202 & CAS6B & 24.2802 & H & 93.20 & u & & A & st & HOM & U02F2 & U235 \\
\hline 203 & CASEO & 24.2734 & H & 93.20 & $u$ & & $A$ & st & HOM & V02F2 & U235 \\
\hline 204 & CASBO & 22.6030 & H & 92.00 & u & & $\hat{A}$ & cr & ном & NITRATE & U235 \\
\hline 205 & CASE1 & 22.6333 & H & 92.60 & $u$ & & $A$ & cr & HOM & NITRATE & U236 \\
\hline 200 & CASE2 & 21.4408 & H & 92.00 & $u$ & & A & $c r$ & HOM & NITAATE & U23E \\
\hline 207 & CASE3 & $21.44 \theta 2$ & H & 92.00 & $u$ & & $A$ & $c r$ & HOM & NITRATE & U236 \\
\hline 208 & CASB4 & 21.4310 & H & 02.00 & $u$ & & A & cr & HOM & NITRATE & U235 \\
\hline 200 & CASBE & 21.4714 & H & 02.00 & u & & A & $\mathrm{cr}$ & HOM & NITRATE & U236 \\
\hline 210 & CAS6O & 21.6547 & H & 92.00 & $\mathbf{P}$ & PLEX & A & $\mathrm{cr}$ & HOM & NITRATE & U236 \\
\hline 211 & CAS67 & 21.8148 & H & 92.00 & $\mathbf{P}$ & PLEX & A & CY & HOM & NITAATE & U236 \\
\hline 212 & CASBB & 21.7361 & H & 92.80 & $\mathbf{P}$ & PLEX & A & $c r$ & ном & NITRATE & U236 \\
\hline 213 & CASEO & 22.1176 & H & 92.60 & $\mathbf{R}$ & PARA & $A$ & cr & HOM & NITAATE & U236 \\
\hline 214 & CAS7O & 21.8888 & H & 82.00 & $\mathbf{P}$ & PARA & A & cr & HоM & NITAATE & U236 \\
\hline 215 & CAST1 & 22.0886 & H & 02.60 & $\mathbf{P}$ & PARA & $A$ & cr & HOM & NITRATE & U236 \\
\hline 210 & CAS72 & 21.6564 & H & 92.00 & $\mathbf{P}$ & PLEX & A & cr & HOM & NITRATE & U236 \\
\hline 217 & CAS73 & 21.0508 & H & 92.00 & $\mathbf{P}$ & PAAA & A & cr & HOM & NITAATE & U236 \\
\hline .218 & CAS74 & & H & 92.60 & $\mathbf{R}$ & PARA & A & cr & HOM & NITAATE & U236 \\
\hline 210 & CAS76 & 21.0760 & H & 92.00 & $\mathbf{P}$ & PARA & A & cr & HOM & NITAATE & U236 \\
\hline 220 & CAS7O & 21.6042 & H & 92.60 & $\mathbf{P}$ & PLEX & A & cr & HOM & NITAATE & U236 \\
\hline 221 & CAS77 & 22.1254 & $H$ & 92.60 & $\mathbf{P}$ & PLEX & A & CY & HOM & NITAATE & U235 \\
\hline 222 & CAS78 & 22.1254 & $H$ & 92.00 & A & PLEX & A & Cr & HOM & NITAATE & U235 \\
\hline 223 & CAS79 & 21.8220 & H & 92.00 & $\mathbf{P}$ & PLEX & A & $\mathrm{cr}$ & HOM & NITRATE & U236 \\
\hline 224 & CASBO & 21.8683 & H & 02.40 & $\mathbf{P}$ & PLEX & A & cr & HOM & NITRATE & U236 \\
\hline 226 & CASBI & 22.0609 & H & 92.00 & $\mathbf{P}$ & PLEX & A & $\mathrm{cr}$ & HOM & NITRATE & U235 \\
\hline 228 & CASB2 & 21.7486 & H & 92.00 & $\mathbf{P}$ & PLEX & $A$ & CY & HOM & NITRATE & U235 \\
\hline 227 & CASB3 & 22.0213 & H & 92.00 & $\mathbf{P}$ & PLEX & A & cr & HOM & NITRATE & U235 \\
\hline 220 & CASBA & 22.1344 & H & 02.60 & $p$ & PLEX & $A$ & cr & HOM & NITRATE & U236 \\
\hline 220 & CASE6 & 21.0070 & H & 02.60 & $\mathbf{P}$ & PLEX & $A$ & cr & HOM & NITRATE & U236 \\
\hline 230 & CASBOB & 21.0748 & H & 02.60 & $\mathbf{P}$ & PARA & A & cr & HOM & NITRATE & U235 \\
\hline 231 & CASB7 & 22.1210 & H & 92.00 & A & PARA & A & cr & ном & NITRATE & U236 \\
\hline 232 & CASBB & 22.0127 & H & 02.00 & $\mathbf{P}$ & PARA & A & CY & Hом & NITAATE & U235 \\
\hline 233 & CASBO & 22.1070 & H & $\$ 2.00$ & $\mathbf{P}$ & PARA & A & cr & Hом & NITRATE & U235 \\
\hline 234 & CASOO & 21.7403 & H & 02.00 & $u$ & & A & cr & ном & NITRATE & U235 \\
\hline 236 & CASOI & 24.6380 & H & 92.60 & $u$ & & $A$ & cr & HOM & NITRATE & U235 \\
\hline
\end{tabular}


Teblw 0.1 (continued)

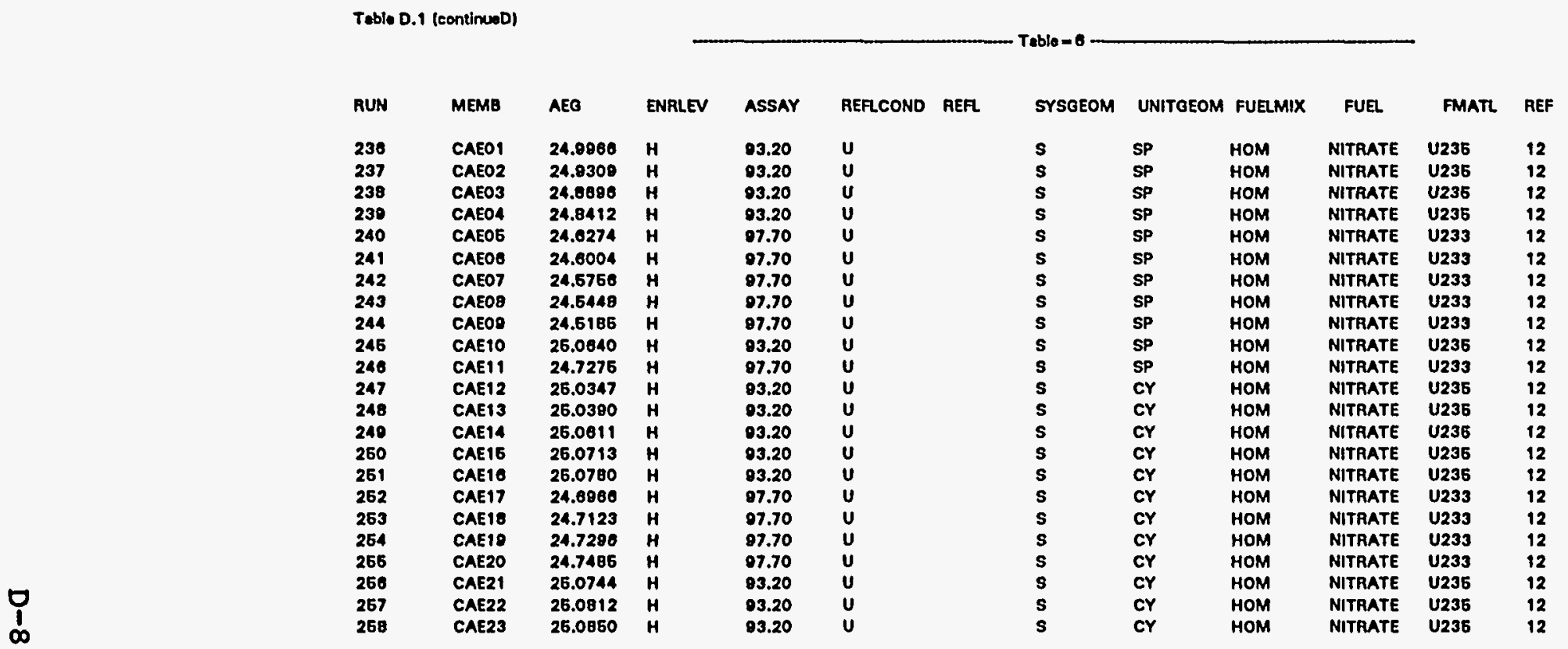




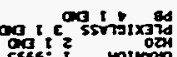

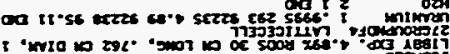

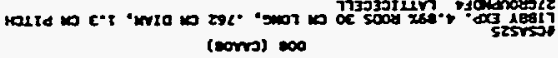

I.

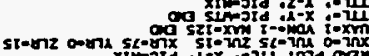

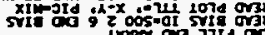

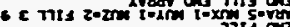

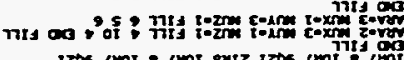

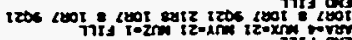

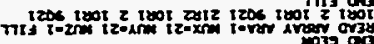

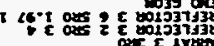

$\operatorname{cosec} 21100$

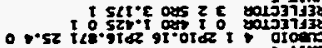
are $s^{2} \sin$

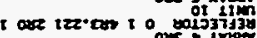

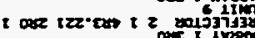

0 c se-doto 0

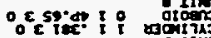

- 6250.412

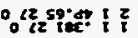

1039 or

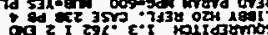

$\log _{t} x_{5}^{\infty} \cos ^{2}$

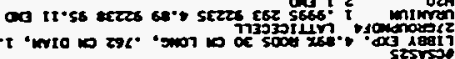
(coms) 40
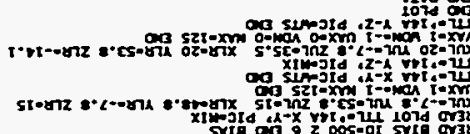

c

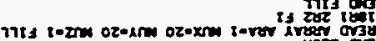

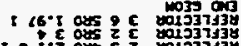

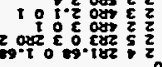

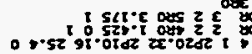

$100 x=10 x-18 z=20$

o 0 so.er 12

ox spide ! !

年1

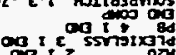

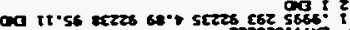

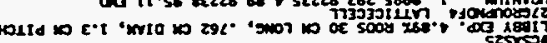

(sonss) 900

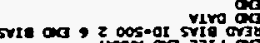

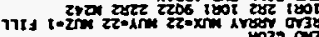

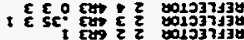

- ox szo.

- of

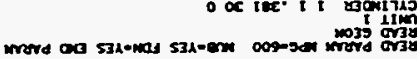

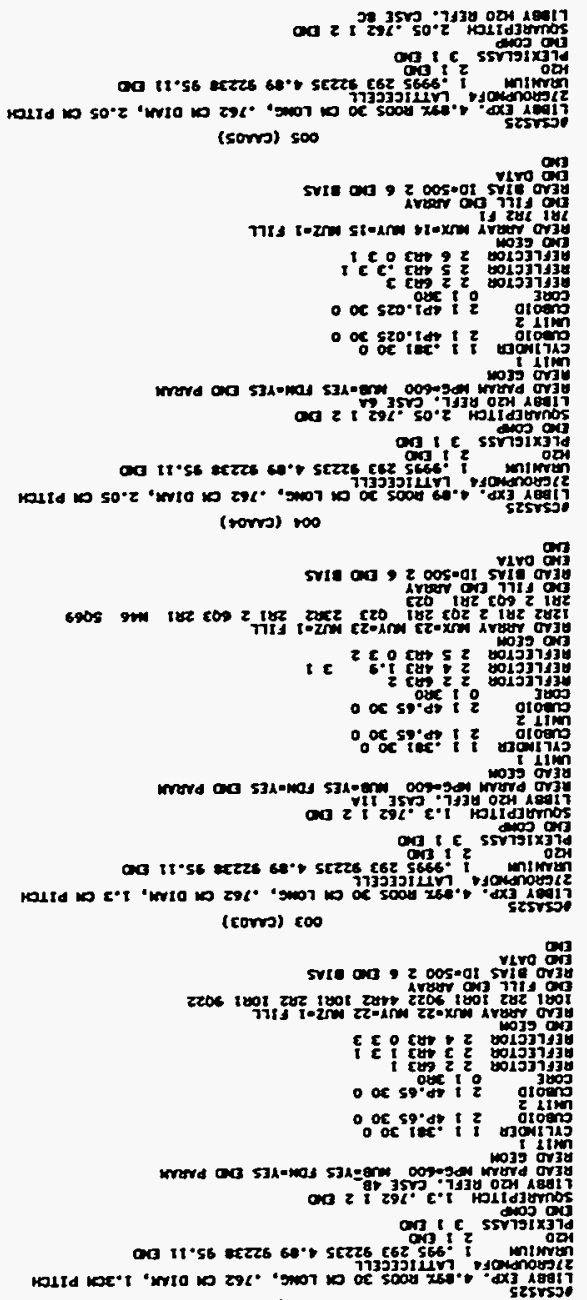
(zorvs) 200

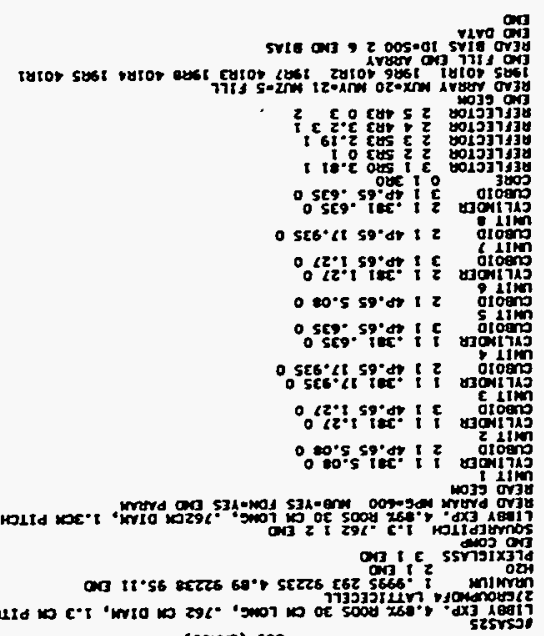
(toms) 100 


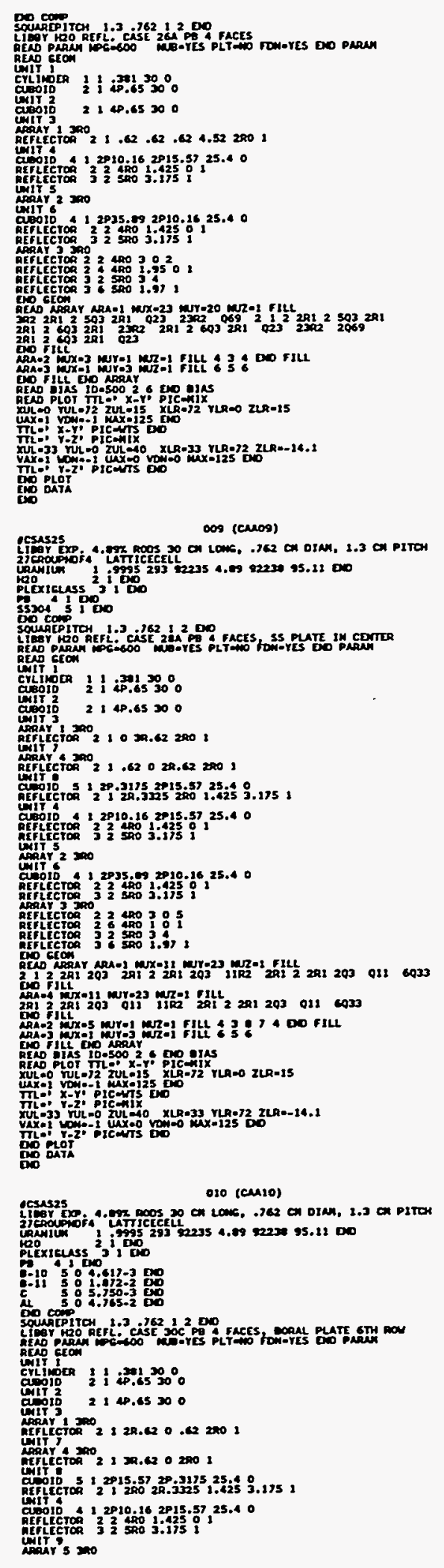

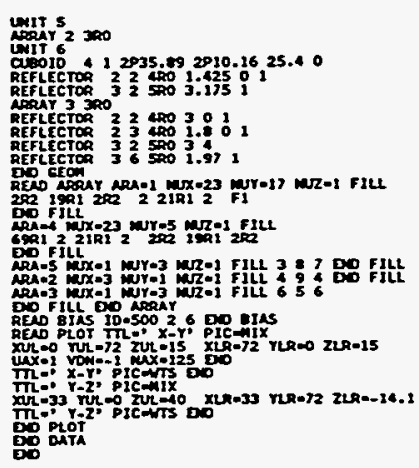

012 (cM12)
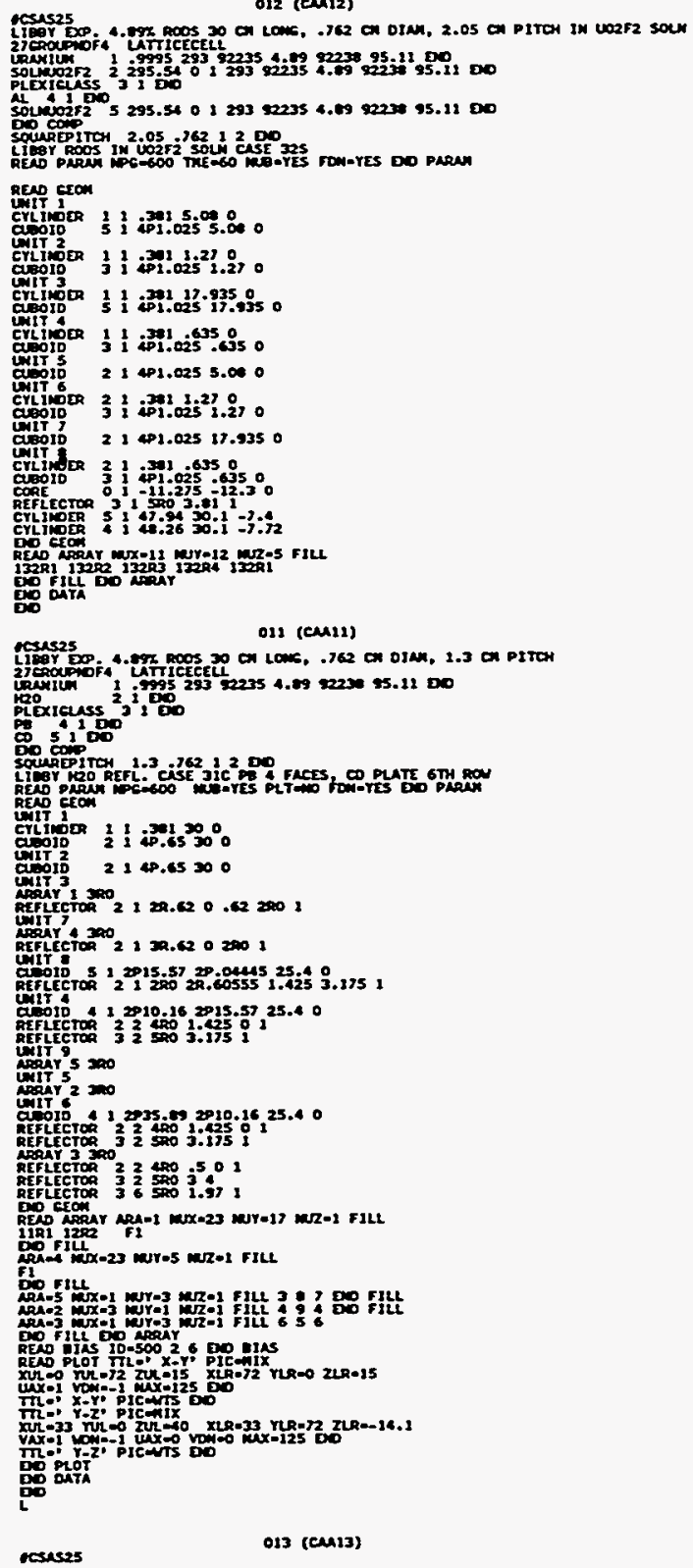


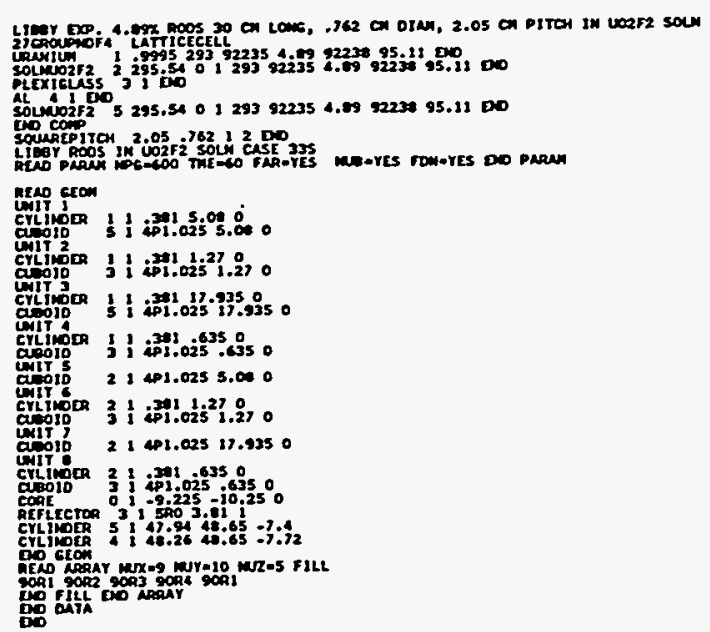

034 (cen14)

CEStes25

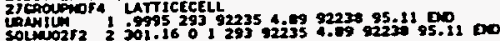

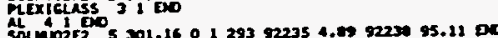

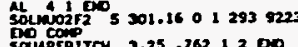

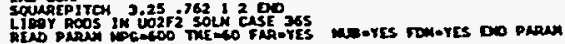

ace crom

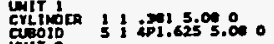

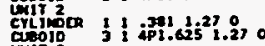

Whit

TII

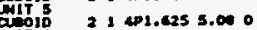

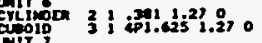

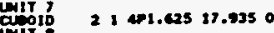

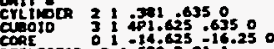

Chy

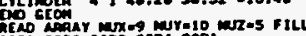

Don

015 (cen15)

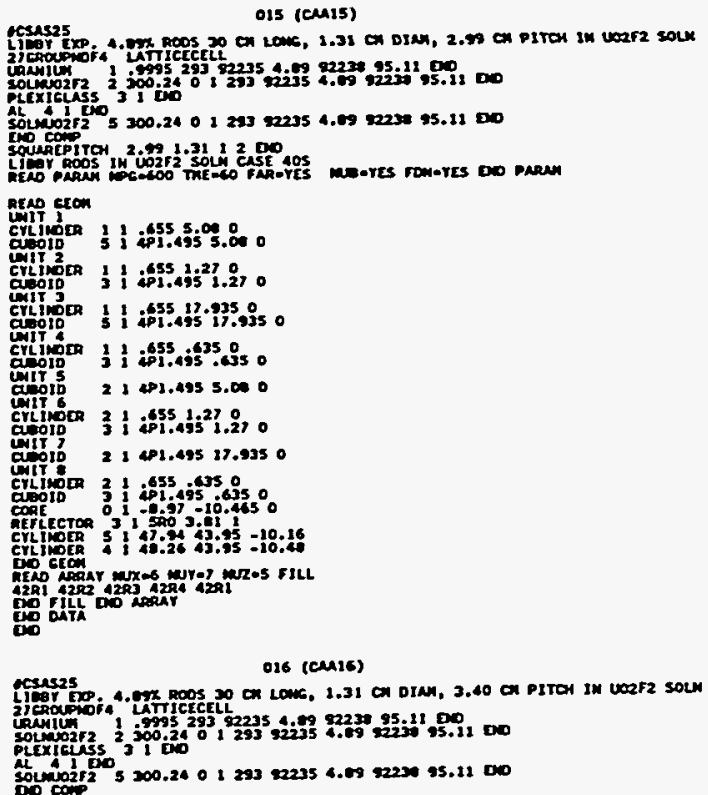

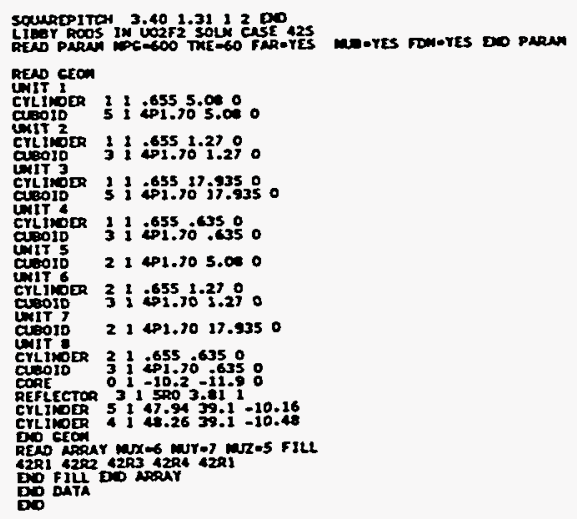

Q17 (cen17)

icses25

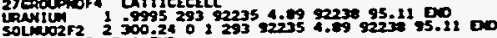

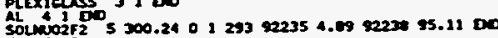

Don

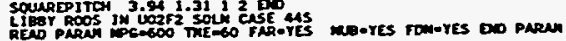

PEDT

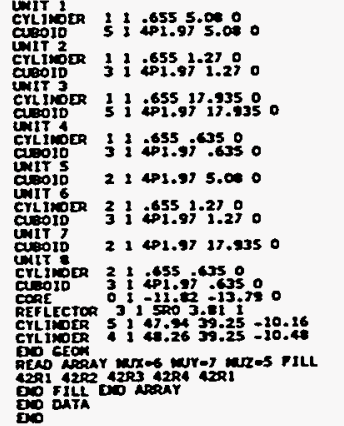

028 (curs)

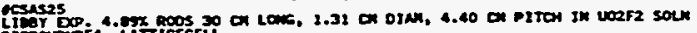

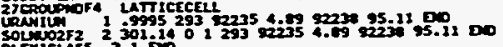

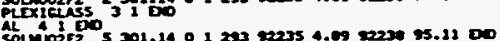

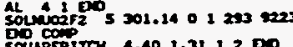

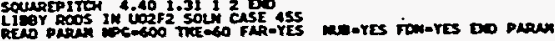

pexp crow

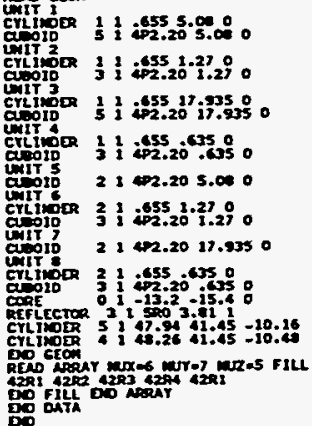

(19) (cen19)

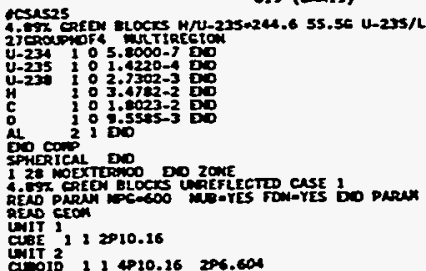



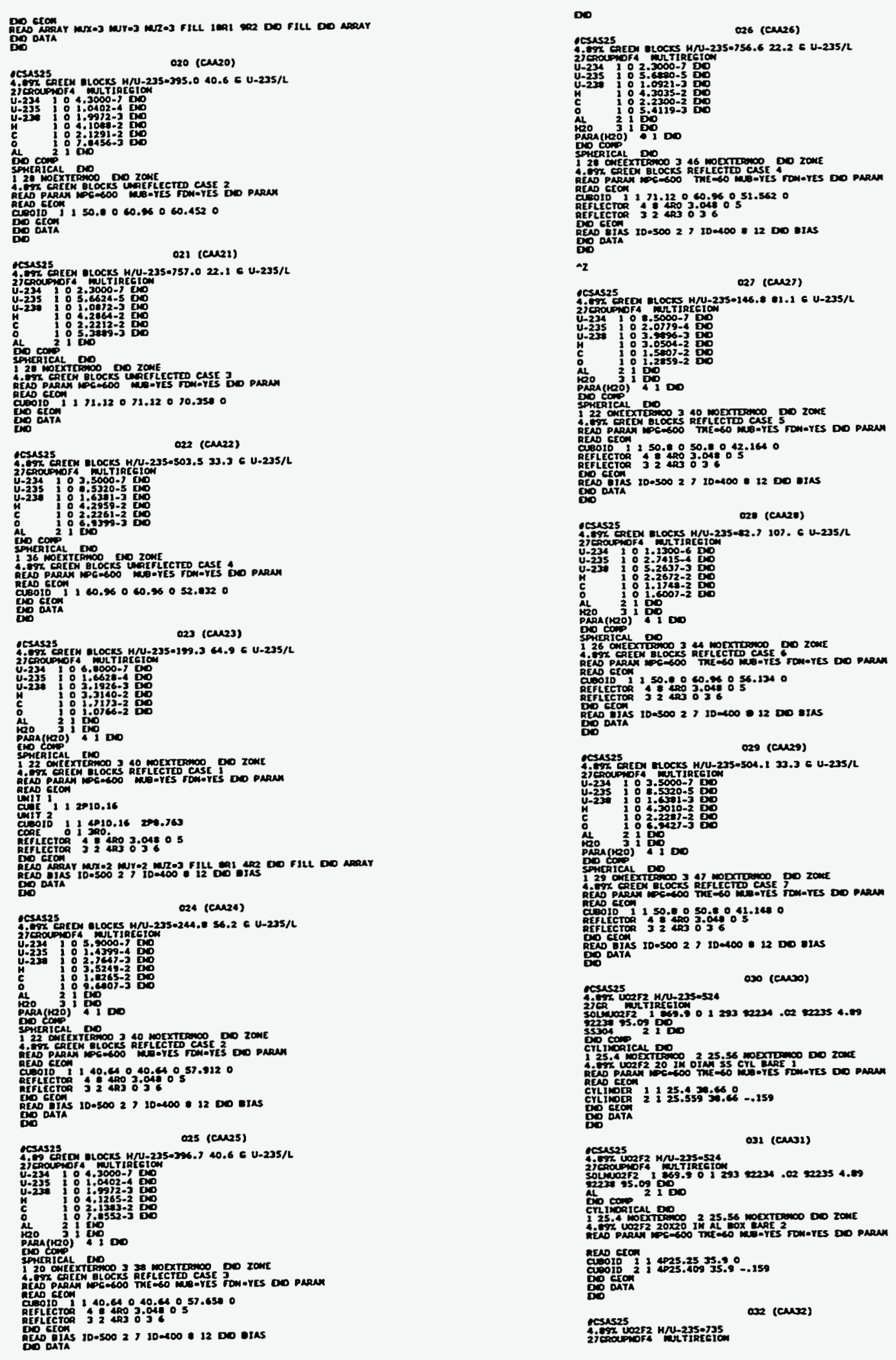

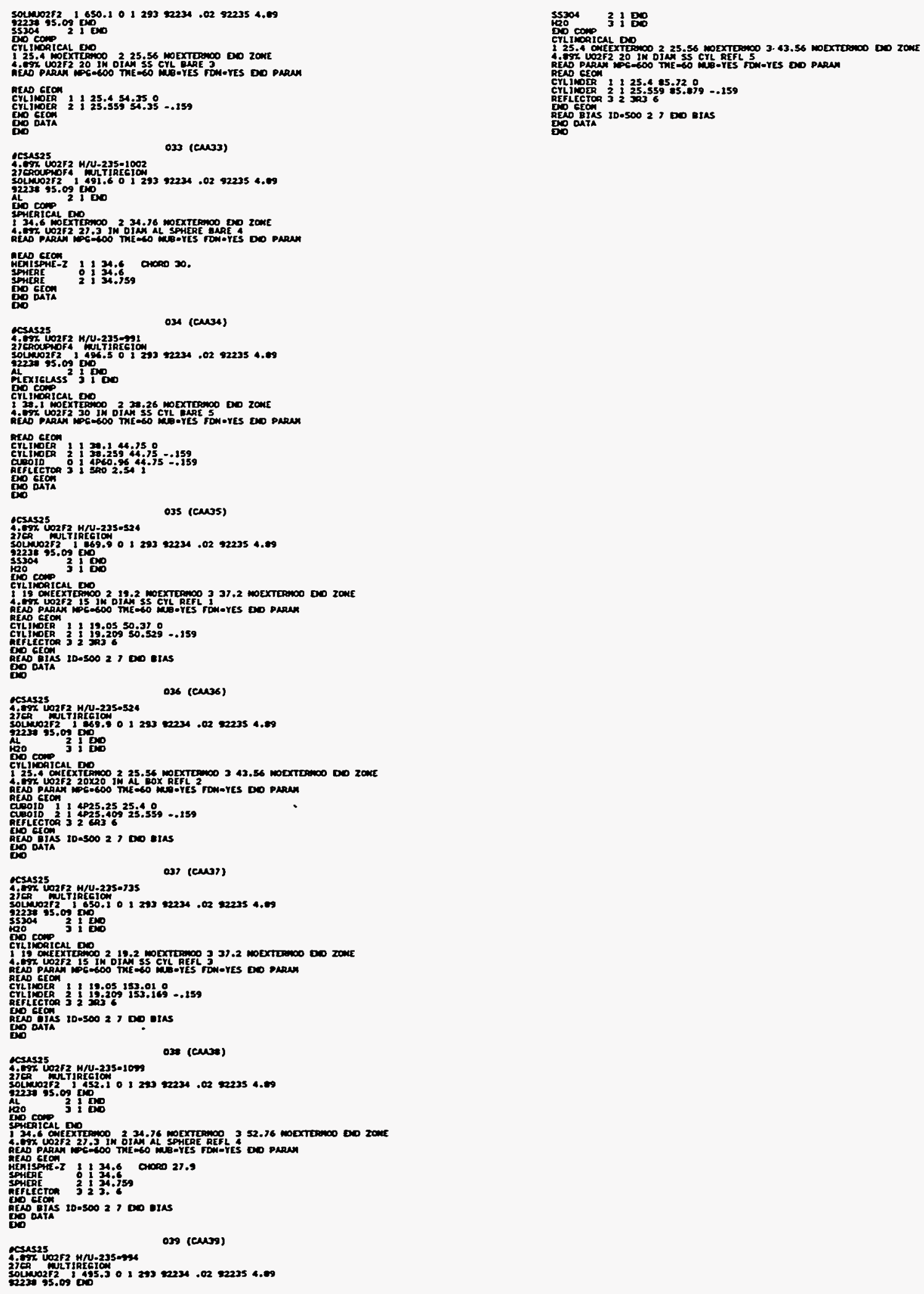


\title{
APPENDIX E2: TABLE 2 INPUTS
}

\author{
080 (Cesol)

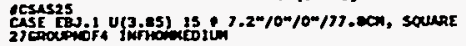 \\ 良.

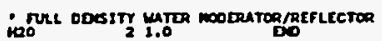

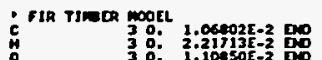

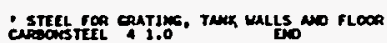

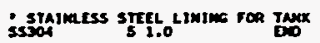

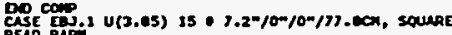 \\ Docesoo PLT

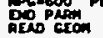

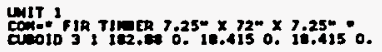

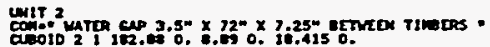

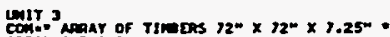

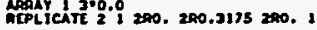

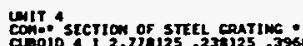

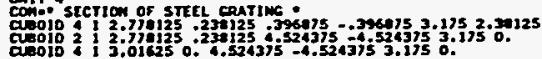 \\ Cull ${ }^{5}$.

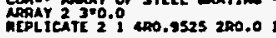

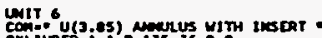

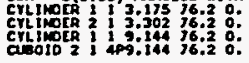

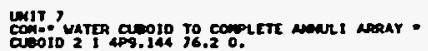

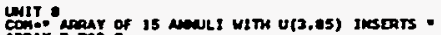

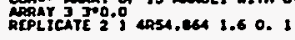

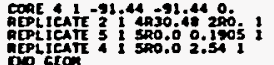

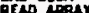 \\ a

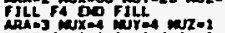 \\ toos

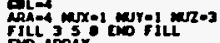 \\ Dein \\ mions

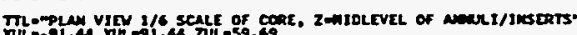

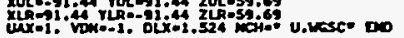

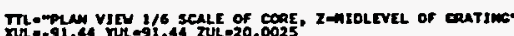

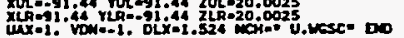

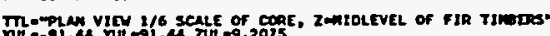

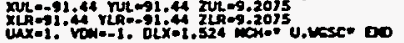

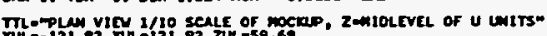 \\ 等

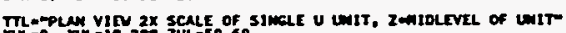

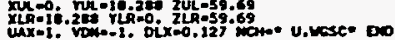

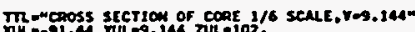

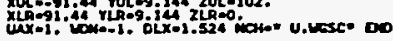

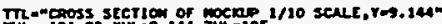

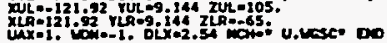

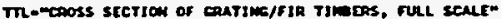

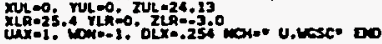

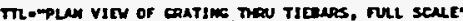

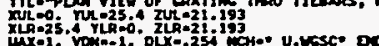

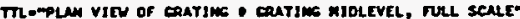

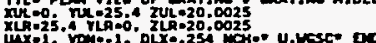

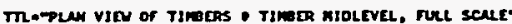

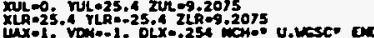

要

OAl (C0002)

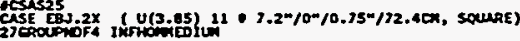

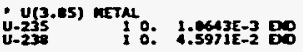

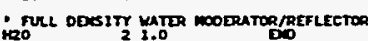

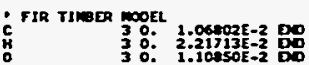

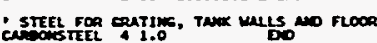

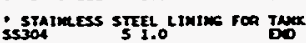

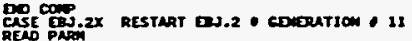

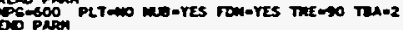

tio cecon

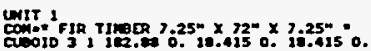

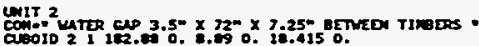

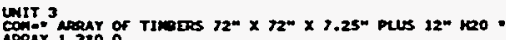

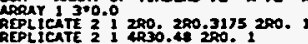

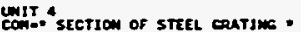

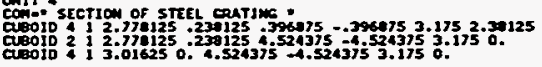

cond 5 "

R⿴囗十

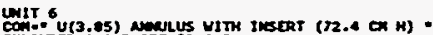

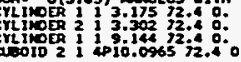

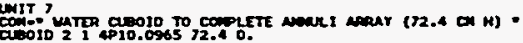

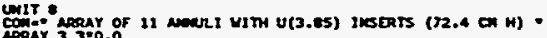
Reptrcht

(4)

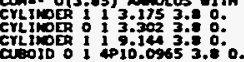

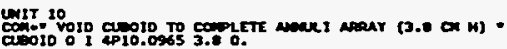

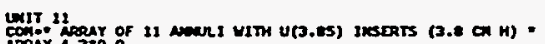

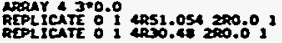

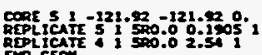

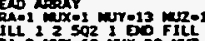

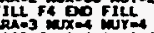

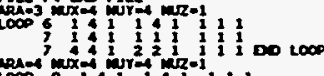

0000

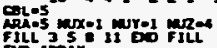

ions

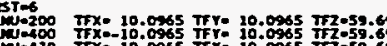

(1)

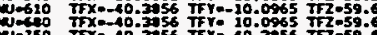

Non

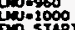

Dio spast

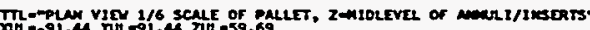

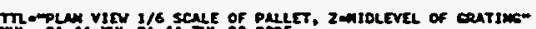

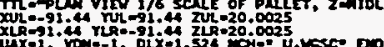

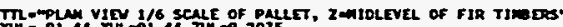

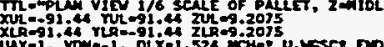

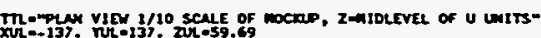




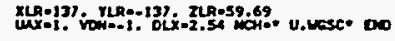

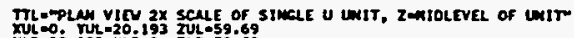

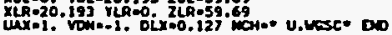

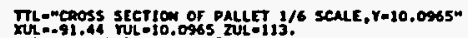

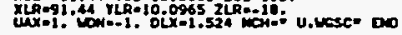

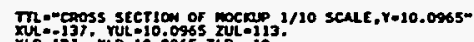

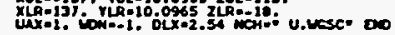

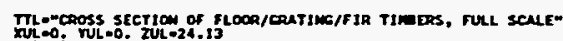

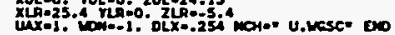

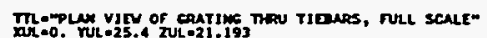

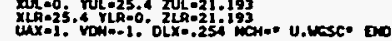

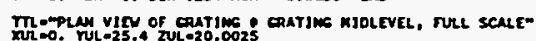

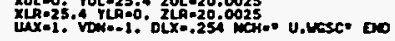

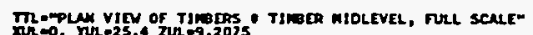
The

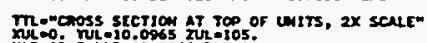

xhe

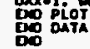

Oar (Cavos)

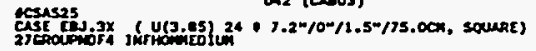

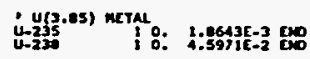

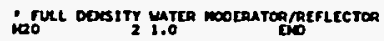

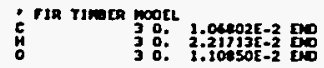

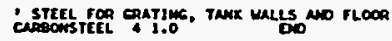

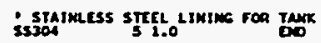

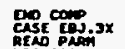

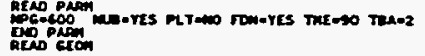

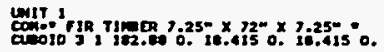

wayr.2

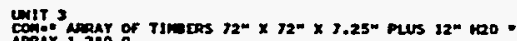

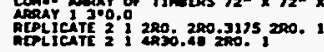

닌.4

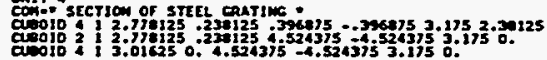

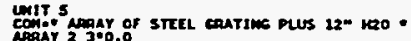

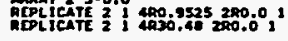

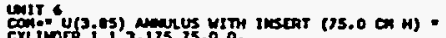

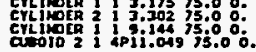

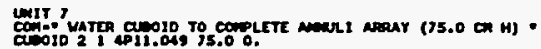

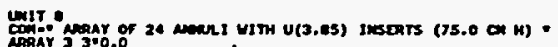

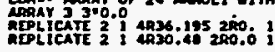

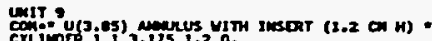

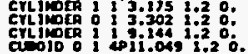

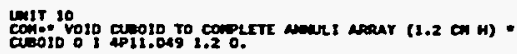

Calt.:"

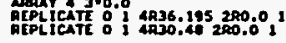

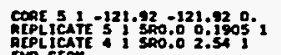

REN

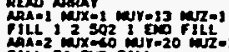

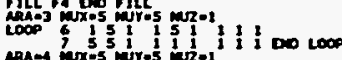

toro-

ches

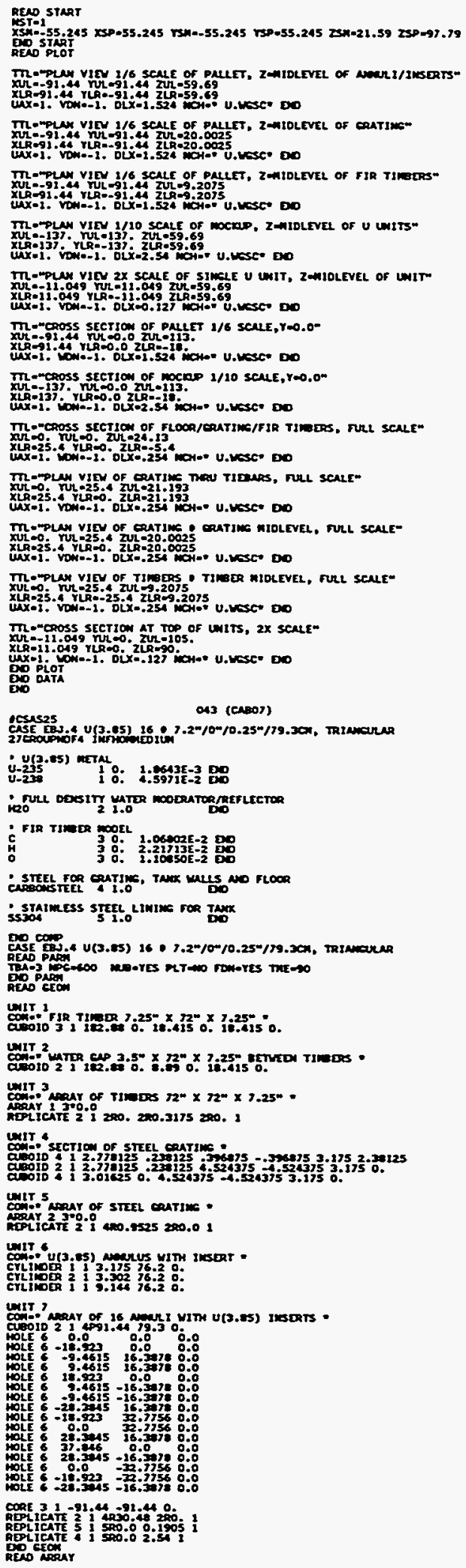

Rens START

Re

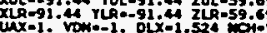

Th-"

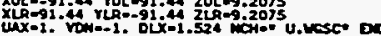

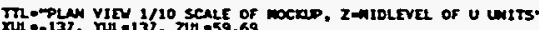

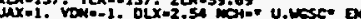

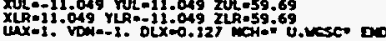

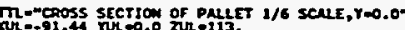

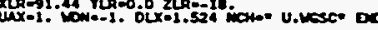

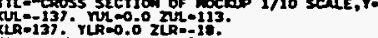

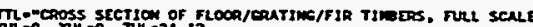

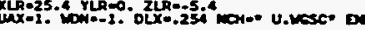

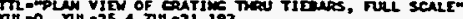

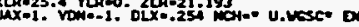

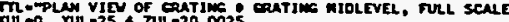

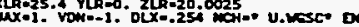

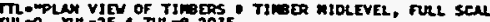

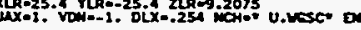

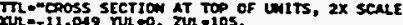

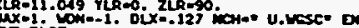

000

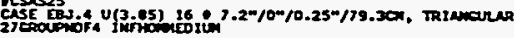

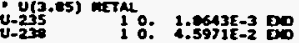

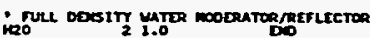

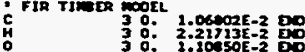

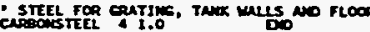

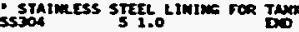

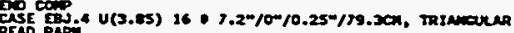

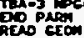

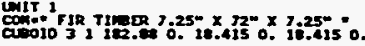

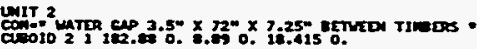

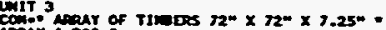

$200.2 \times 0.3175200$.

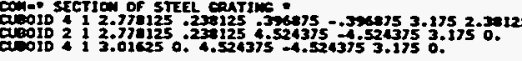

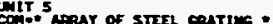

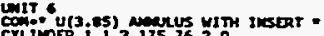

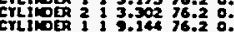

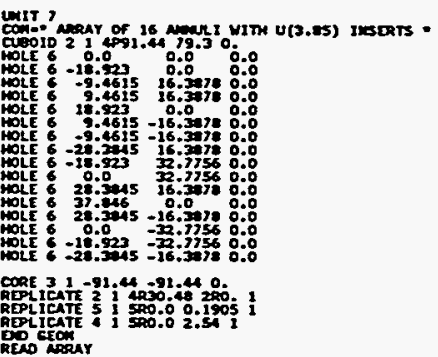




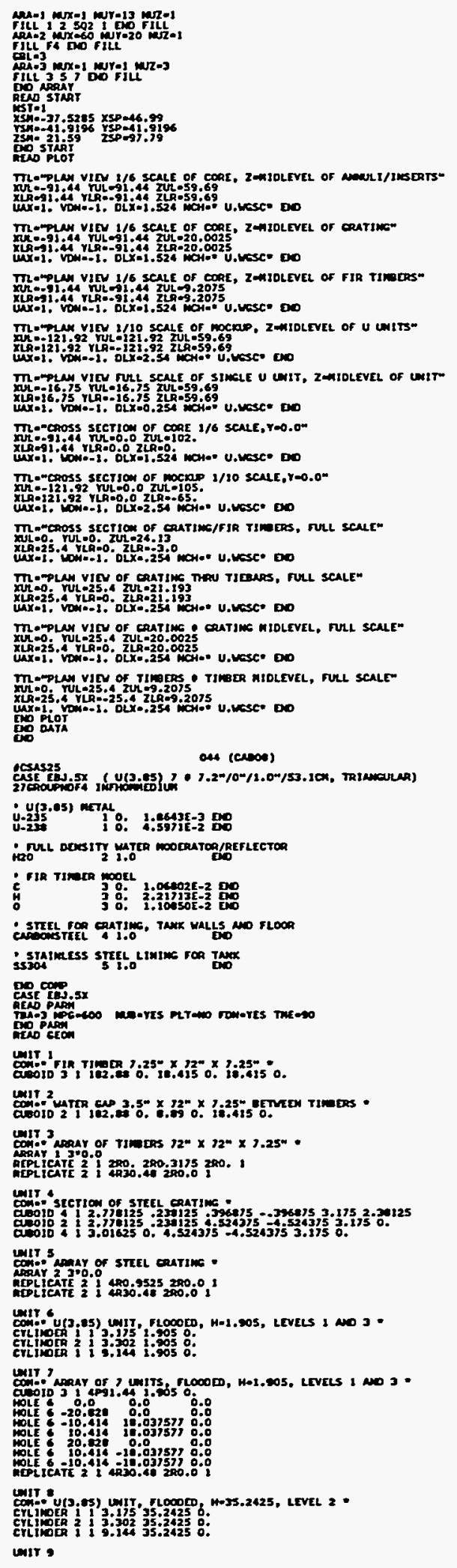

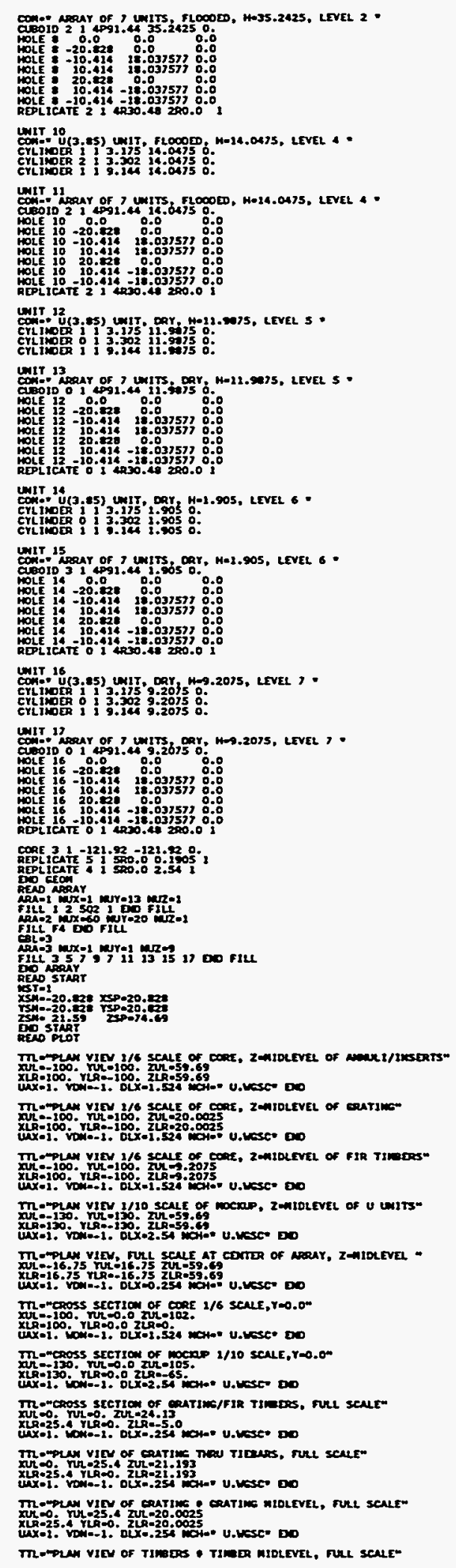




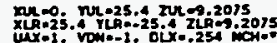

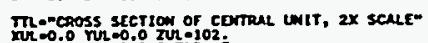

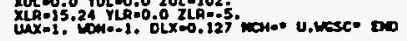
Do por

acs (ccosos)

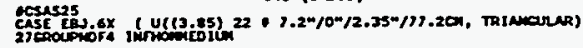

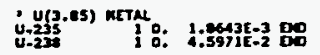

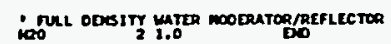

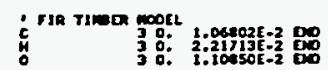

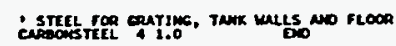

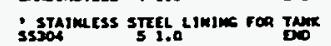

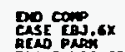

Din

Rud axom

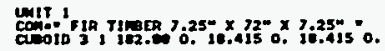

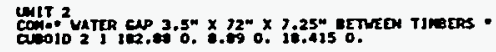

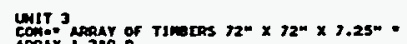

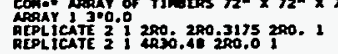

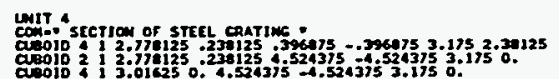

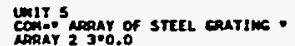

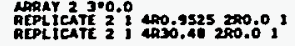

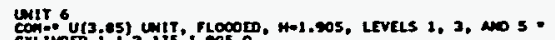

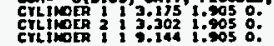

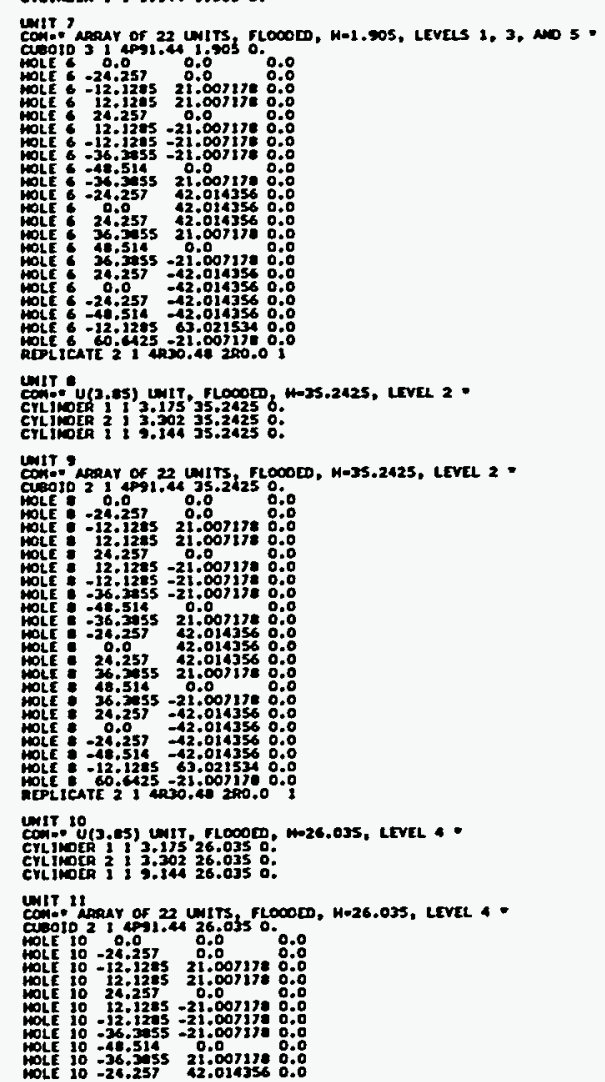

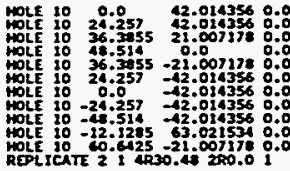

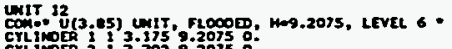
CYLTOE I I 1.3029 .2075 :

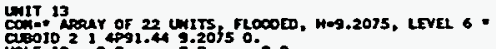

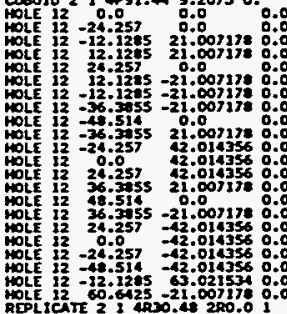

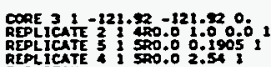

政

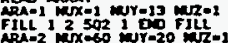

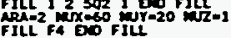

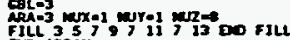

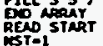

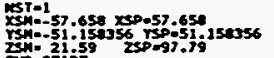

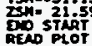

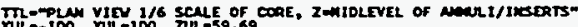

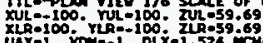

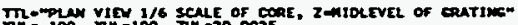
Mr.71

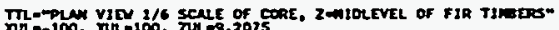

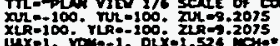

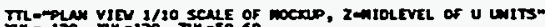
Thin:

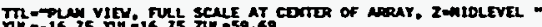

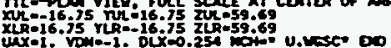

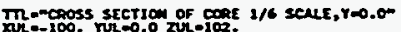

XtLitio.

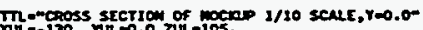

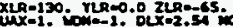

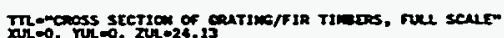

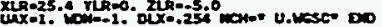

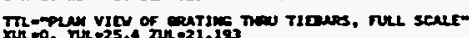

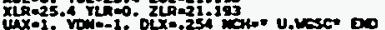

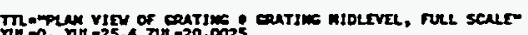

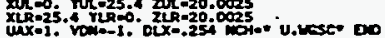

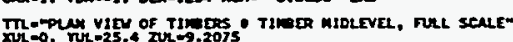

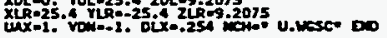

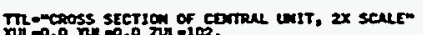

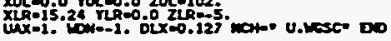
品

Oab (Cov10)

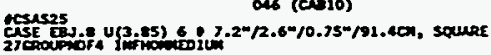

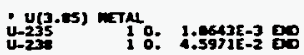

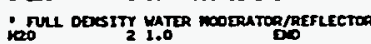

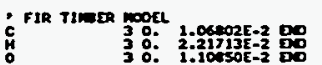

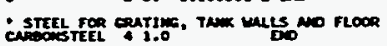

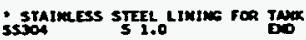




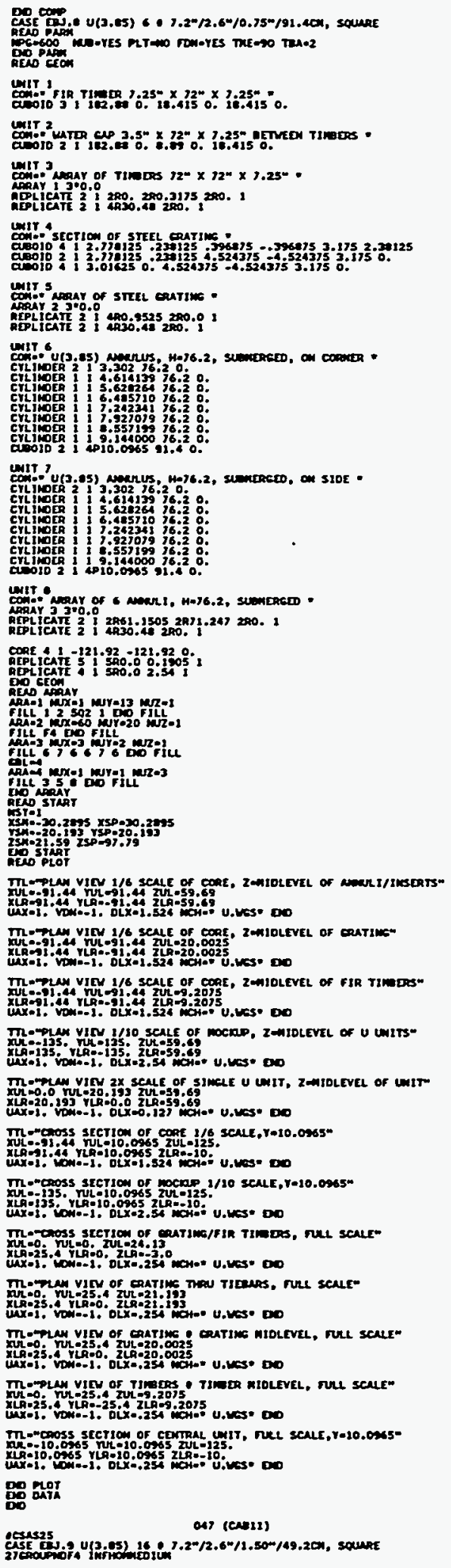




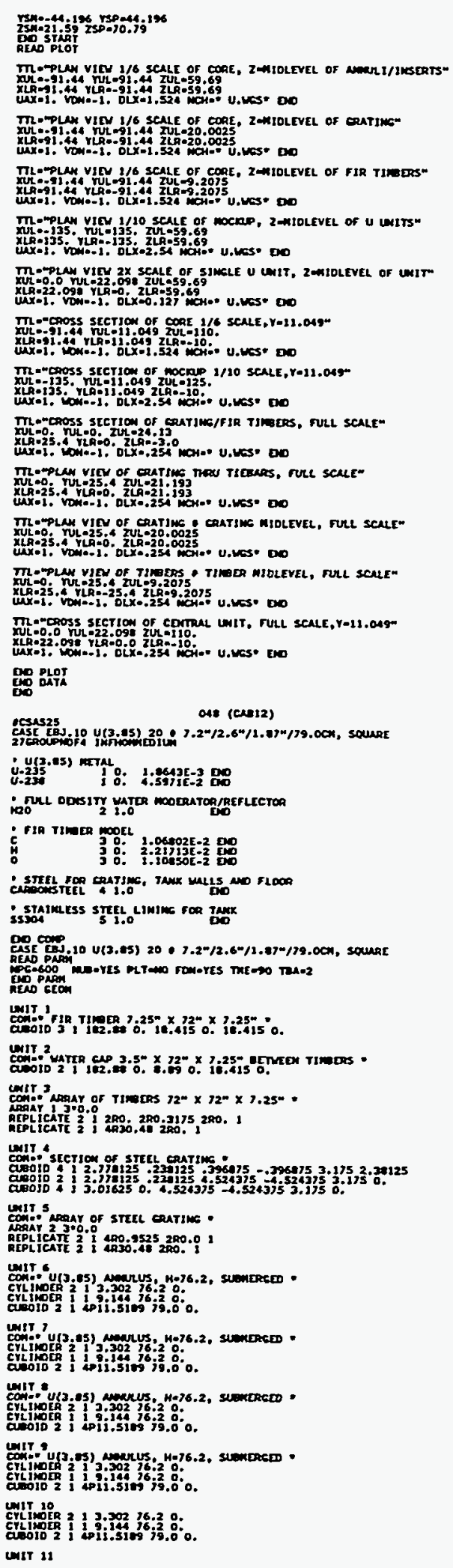

048 (C)es12)

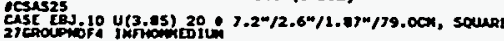

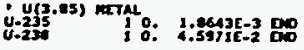

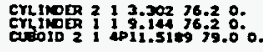

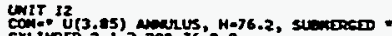

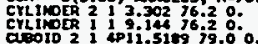

Colt. 13 .

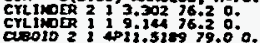

LMIT.14

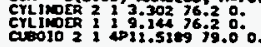

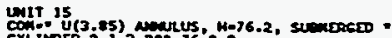

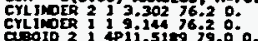

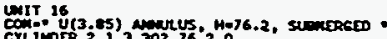

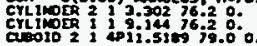

LyII.21)

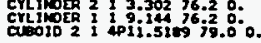

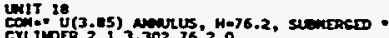

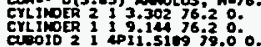

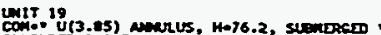

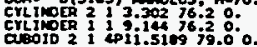

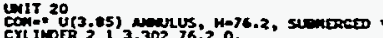

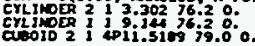

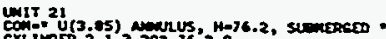

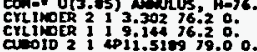

witr 22 (3)

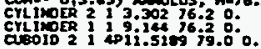

LaIIT.23

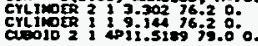

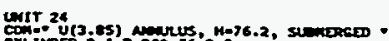

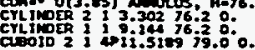

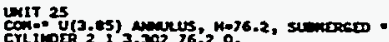

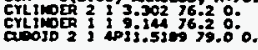
W011.23

coury 27 , RTDL

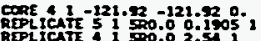

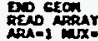

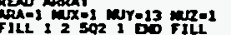

ches

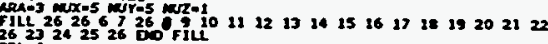

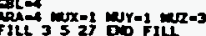

titi

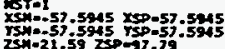

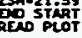

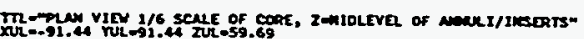

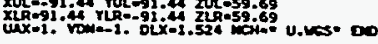

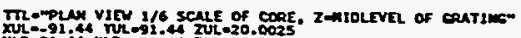

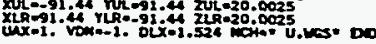

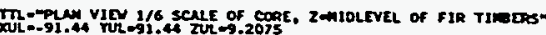

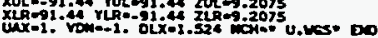

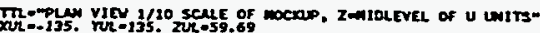

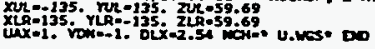

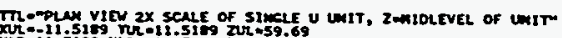

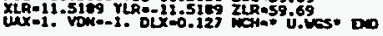

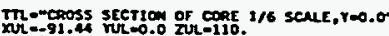

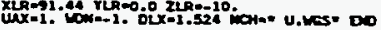

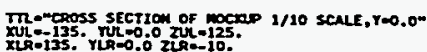




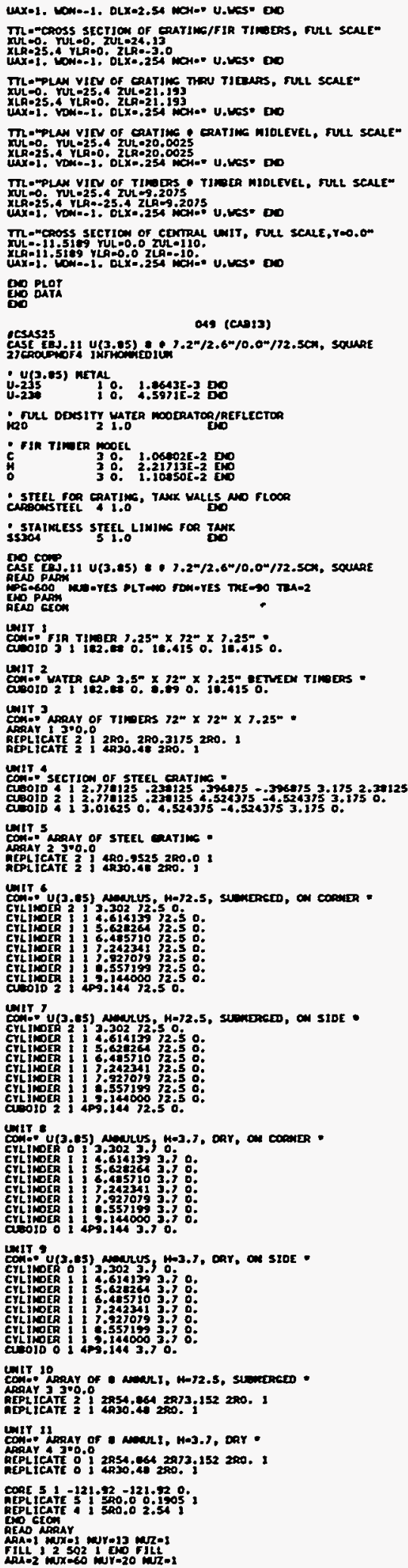

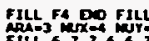

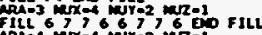

mit.

Mith

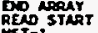

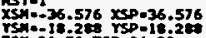

atsot

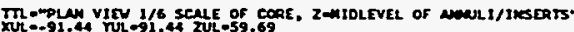

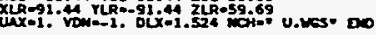

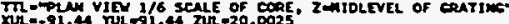

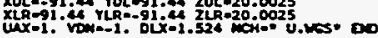

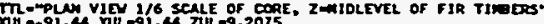

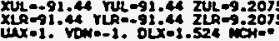

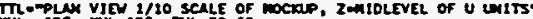

政: 135 .

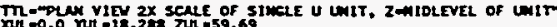

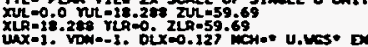

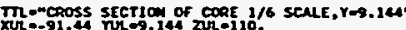

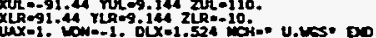

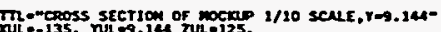

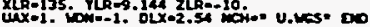

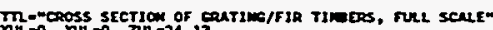

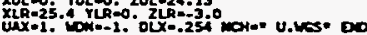

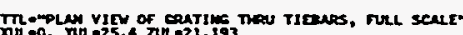

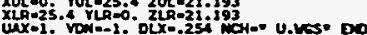

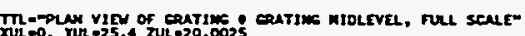

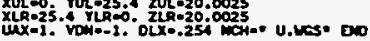

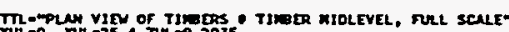

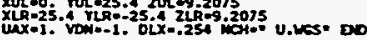

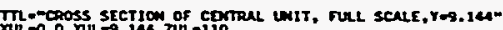

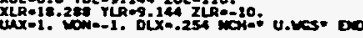

Do por

O50 (c)er14)

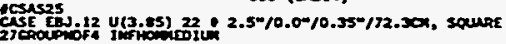

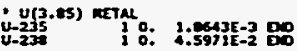

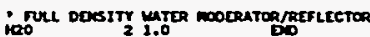

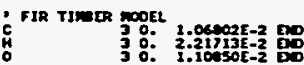

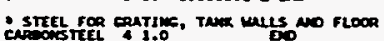

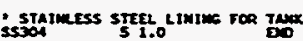

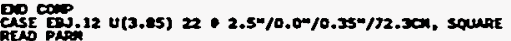

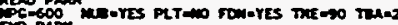

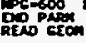

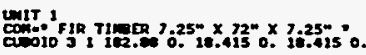

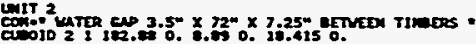

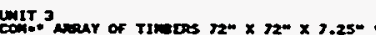

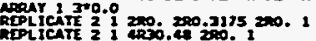

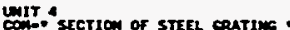

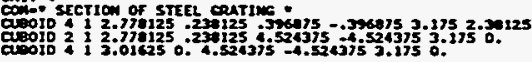

wert.5

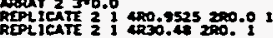

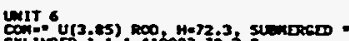

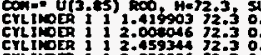

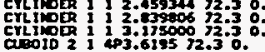

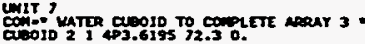

cort."

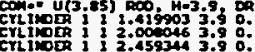




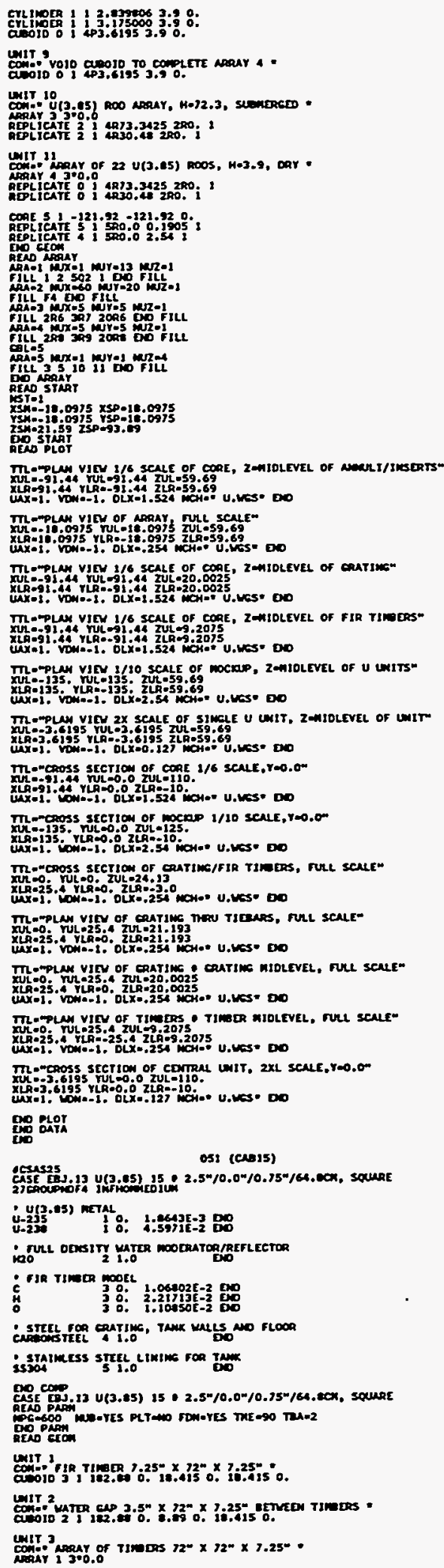

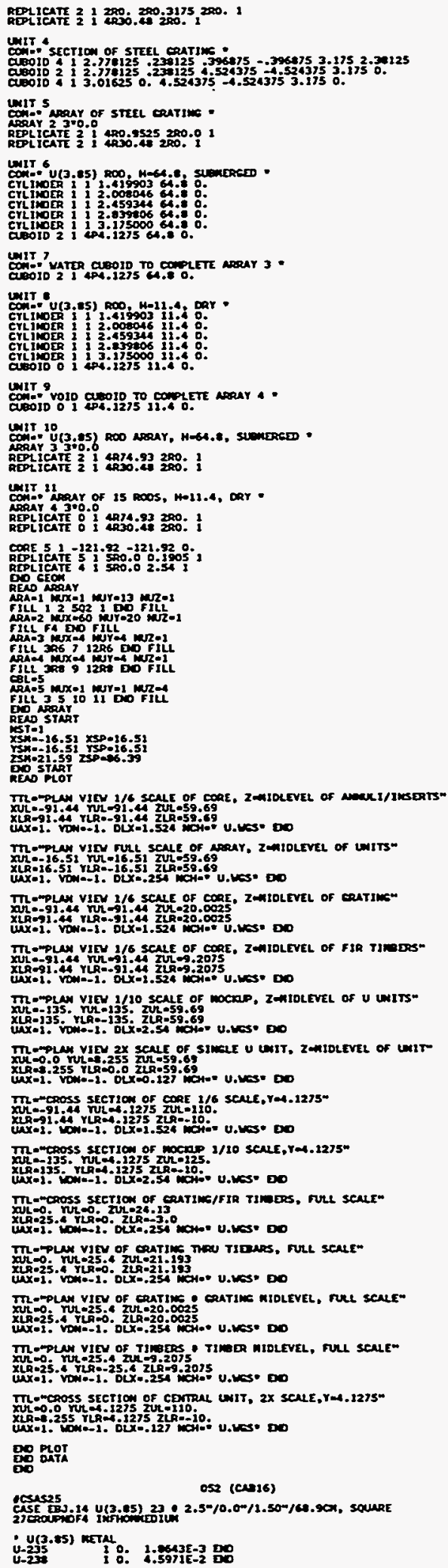




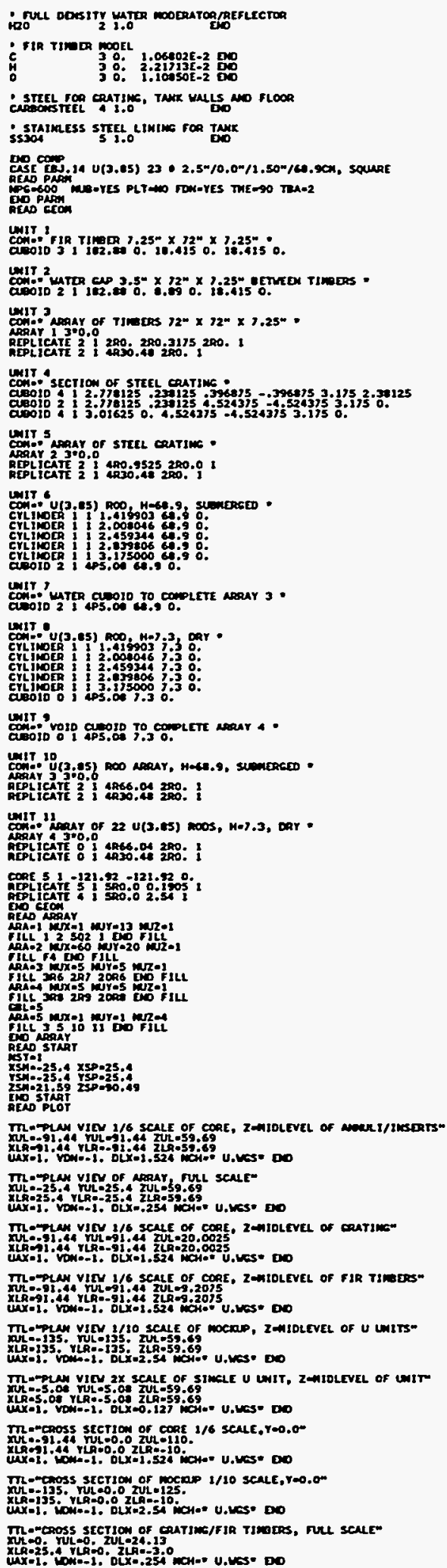

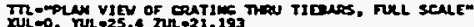

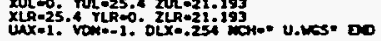

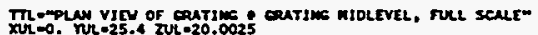
xhy =0. The

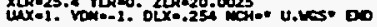

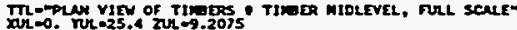

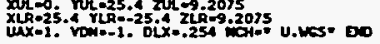

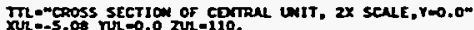

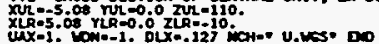

goo gitor 


\section{APPENDIX E3: TABLE 3 INPUTS}

OS3 (casas)

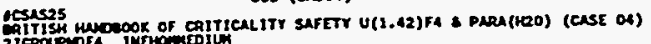

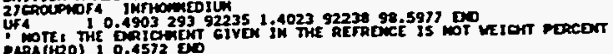
Mas (40)

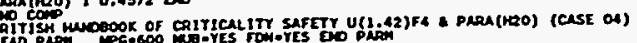
ETCH 2046.552046 .502961 .9 go chom

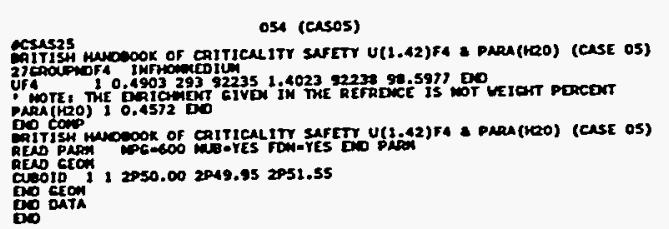

oss (casos)

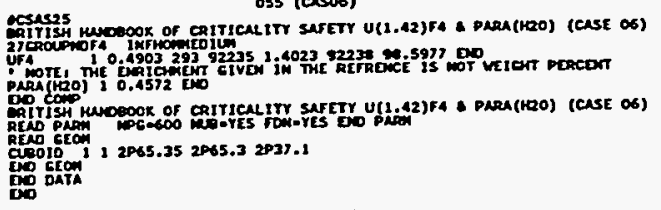

oss (cesi1)

acsess

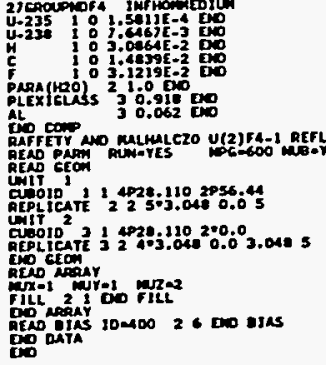

057 (Cas12)

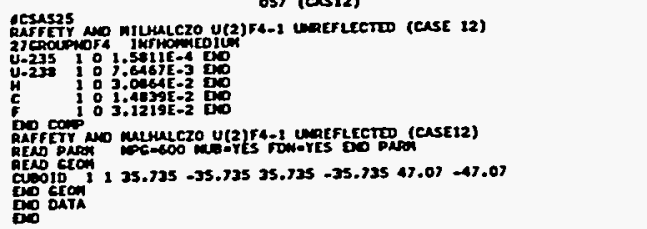

ose (cosis)

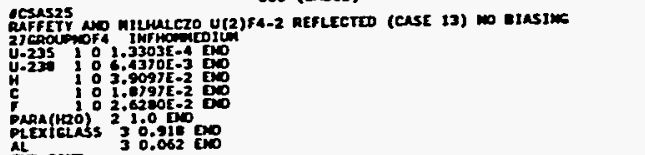

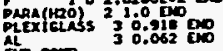

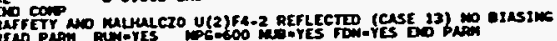

AFDo cen

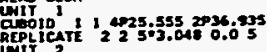

व.⿴囗十

CSTO

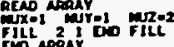

Dom

059 (cessia)

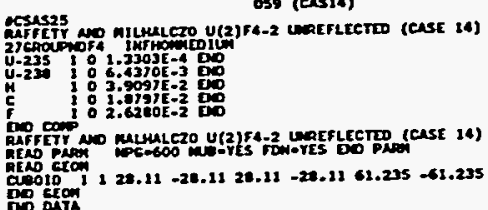

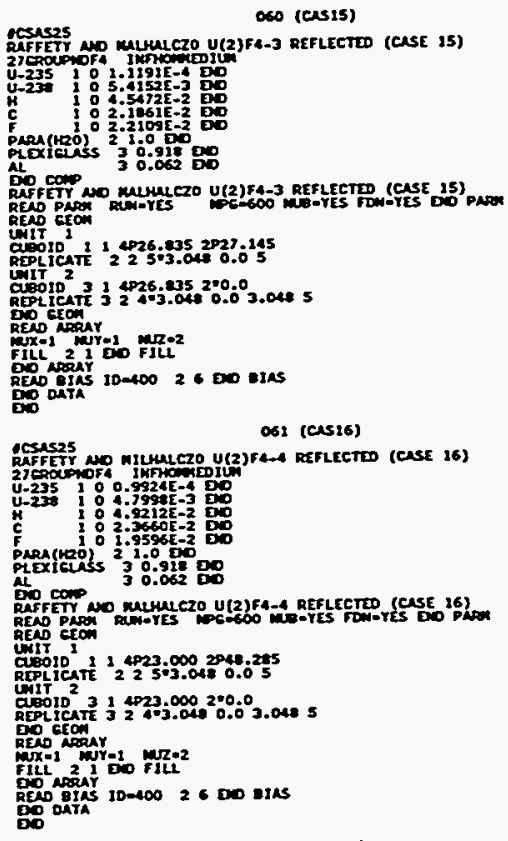
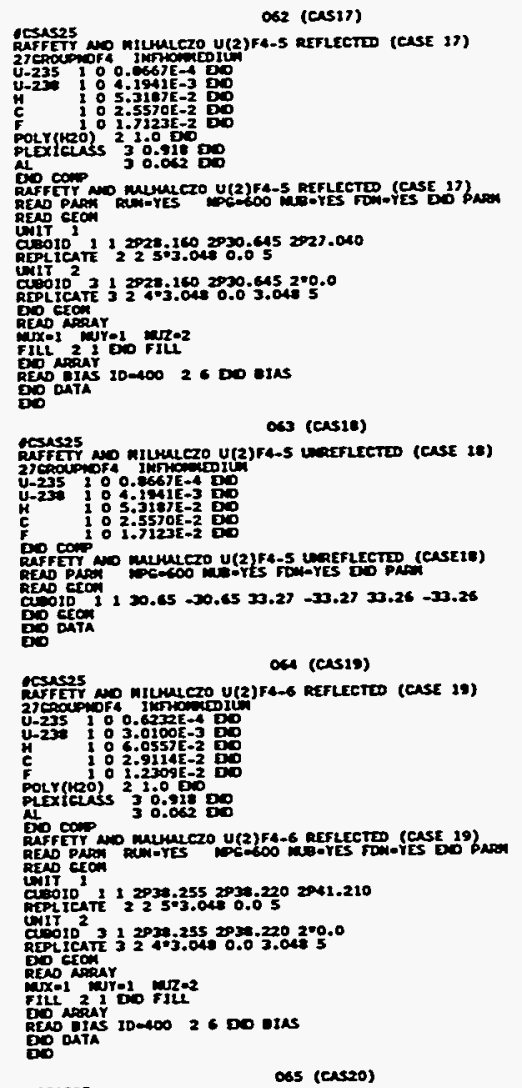

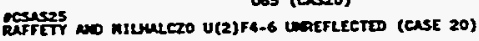



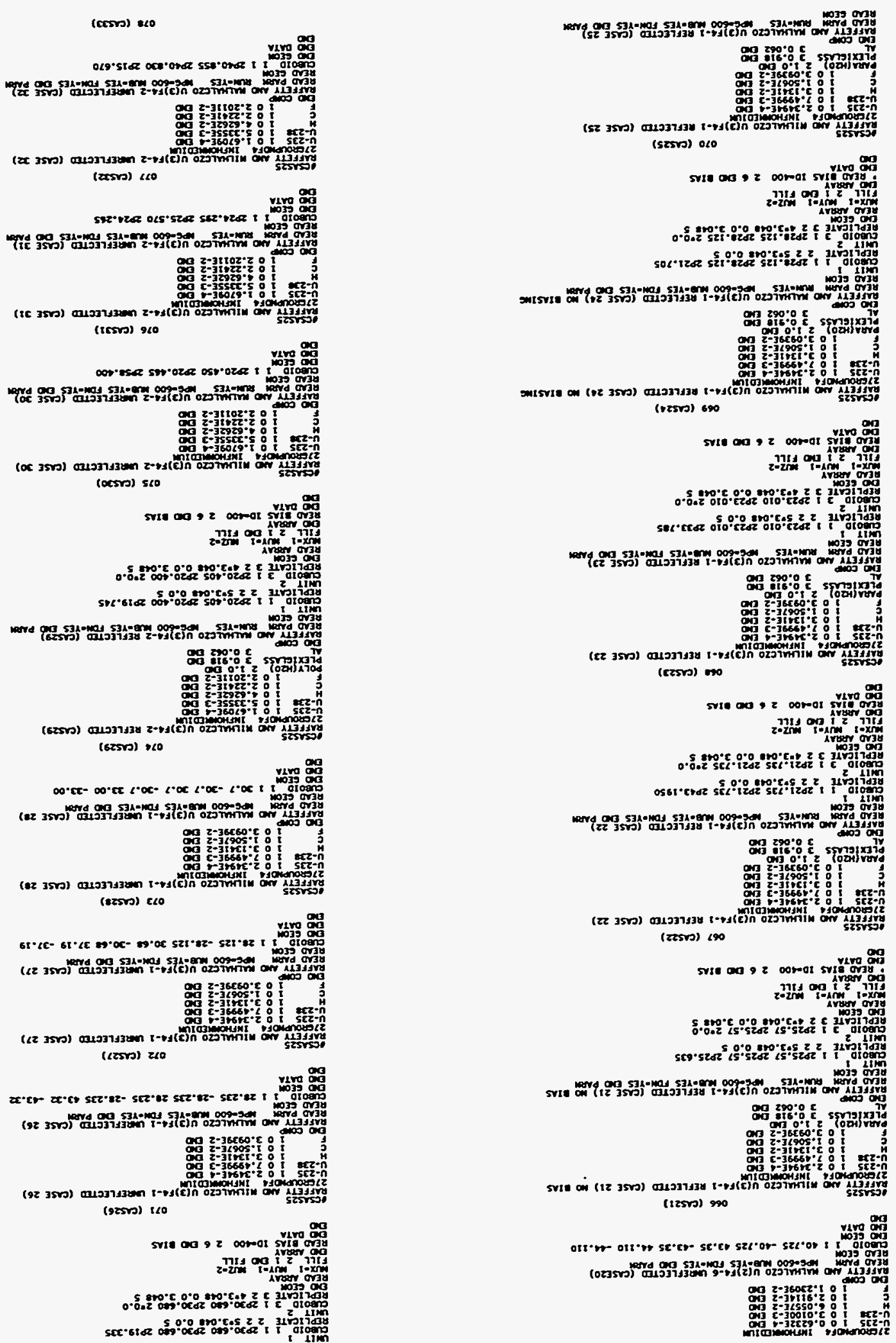


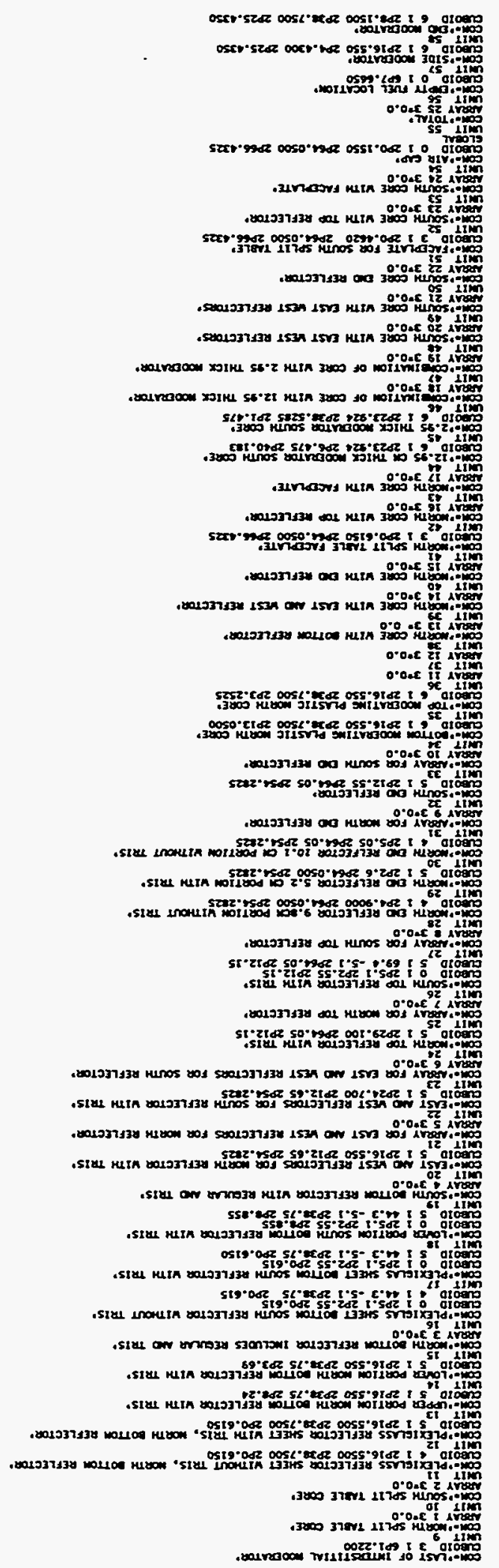

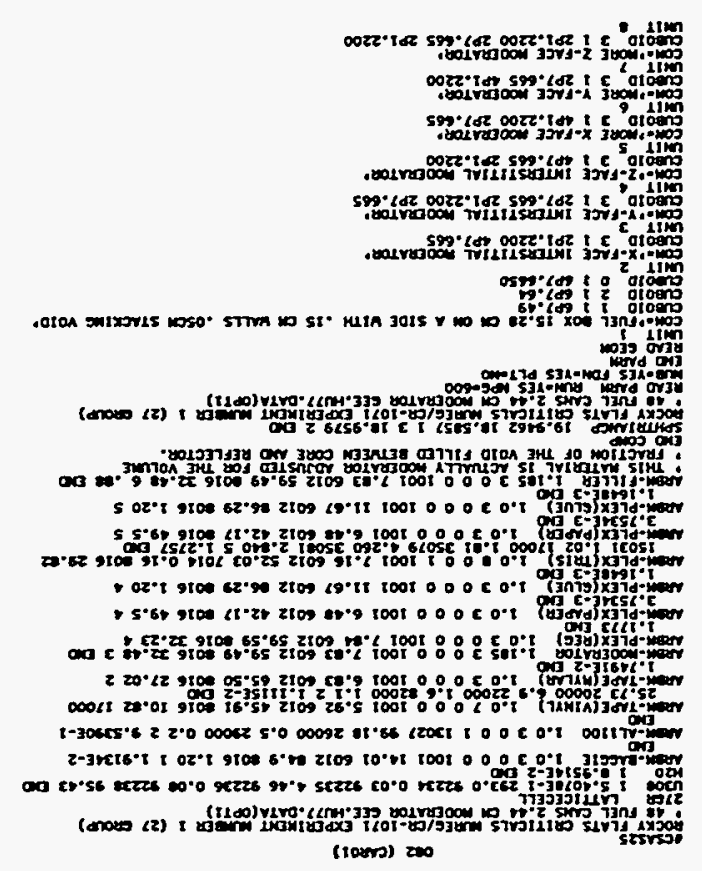

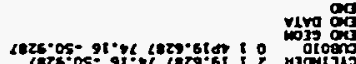

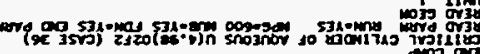

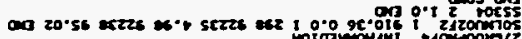

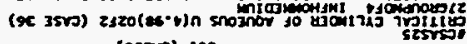
(gesrs) too

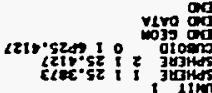

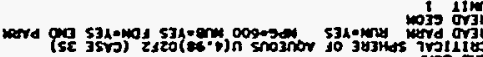
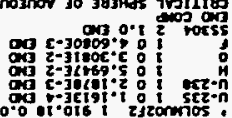

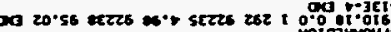

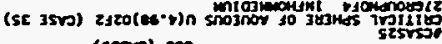
(5)

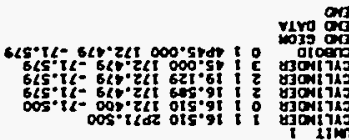

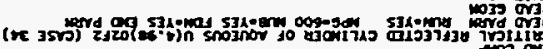

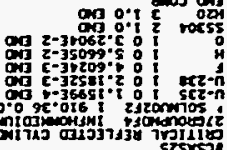

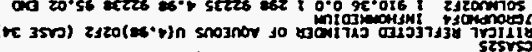
(resso) $8<0$

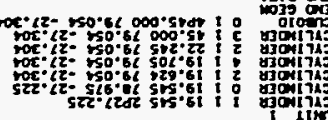

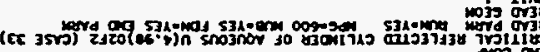

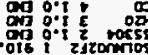

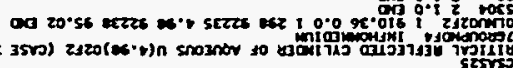




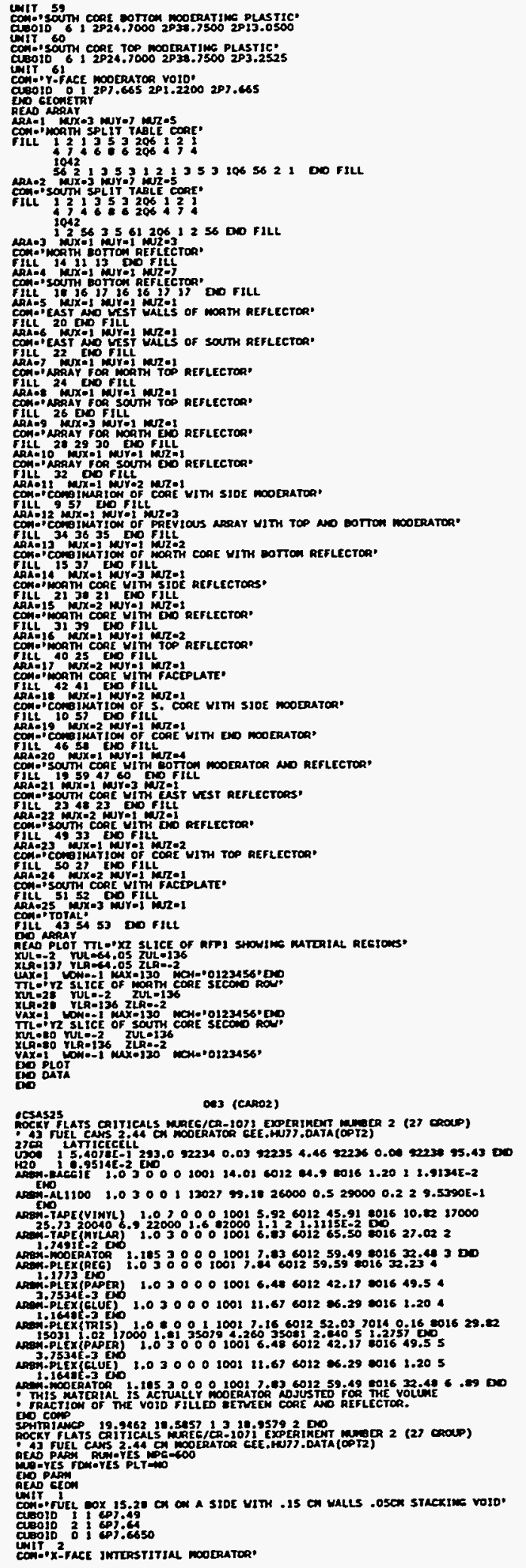

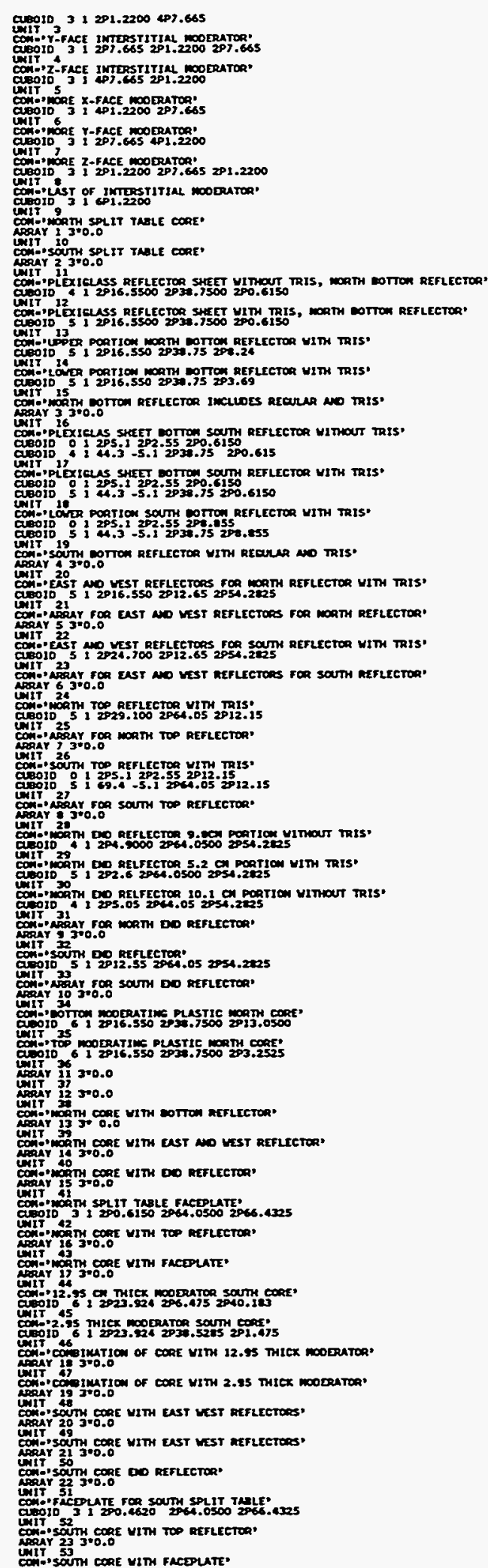




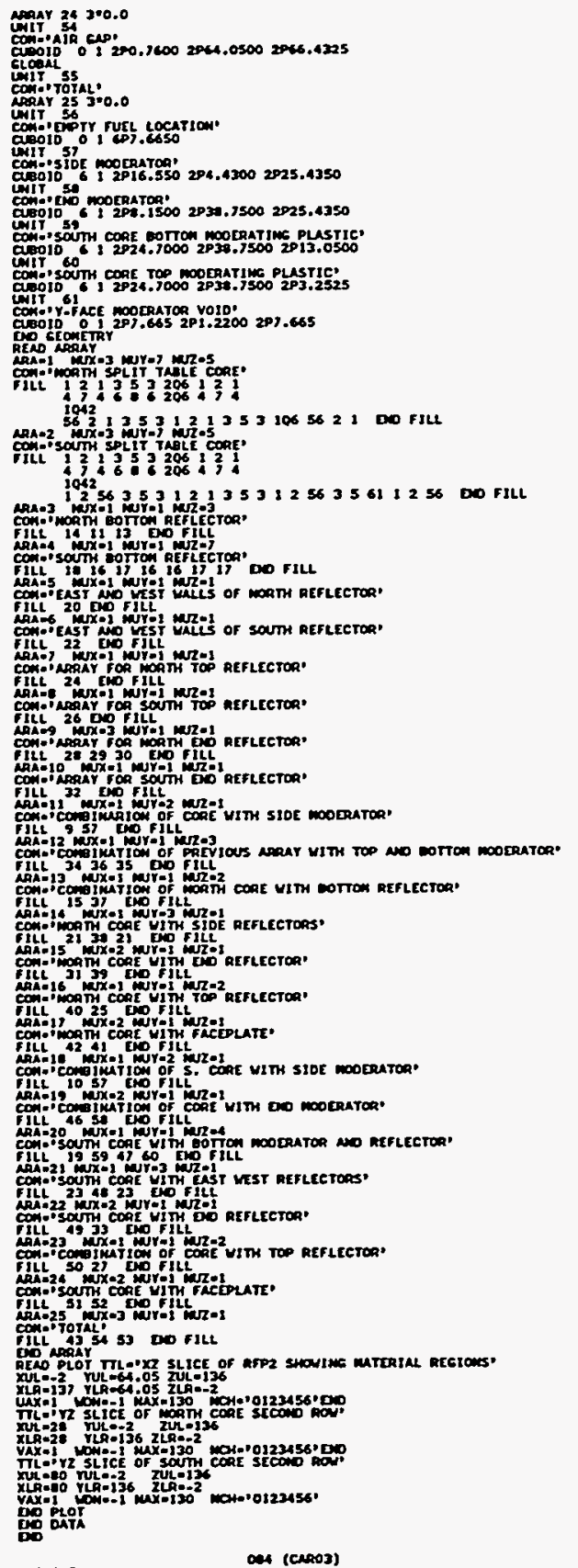

RSeszs

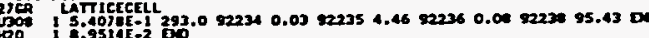
200

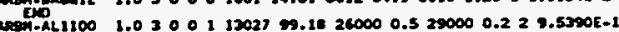

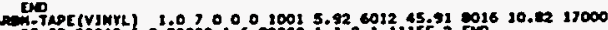

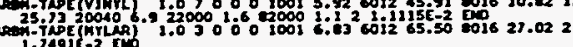

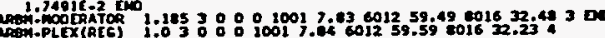

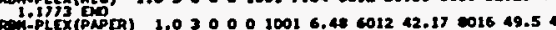
sem:ptixts 1.03000100111 .67001206 .2900161 .20 40.190.

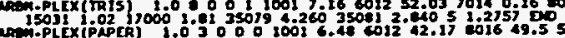

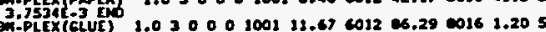

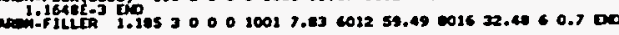

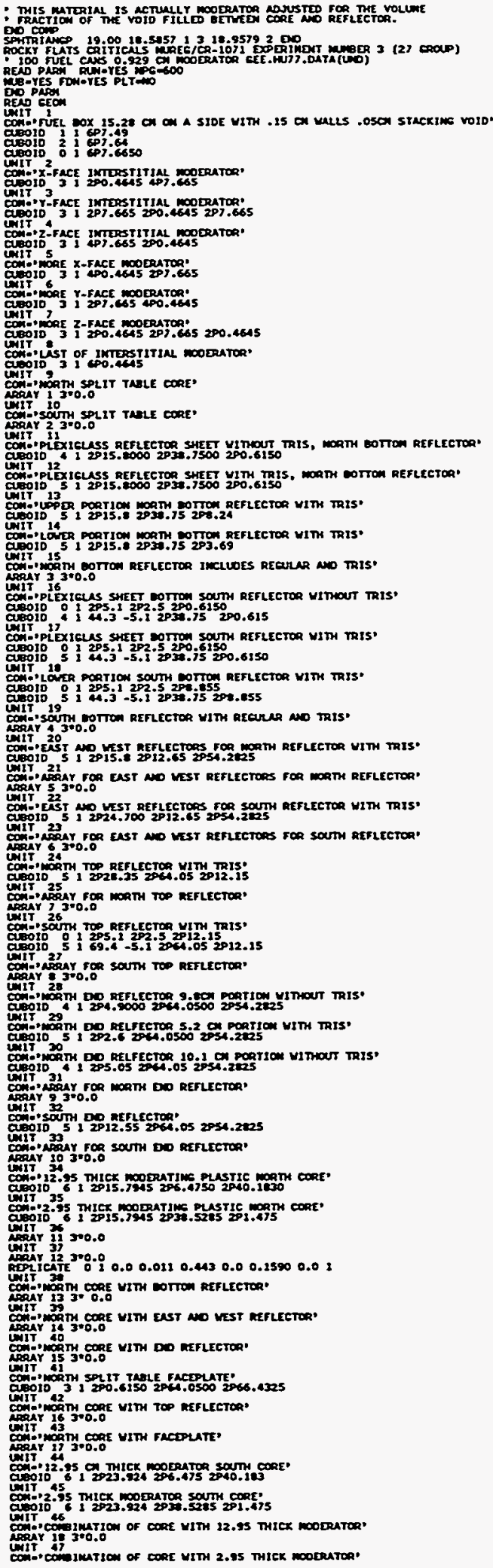




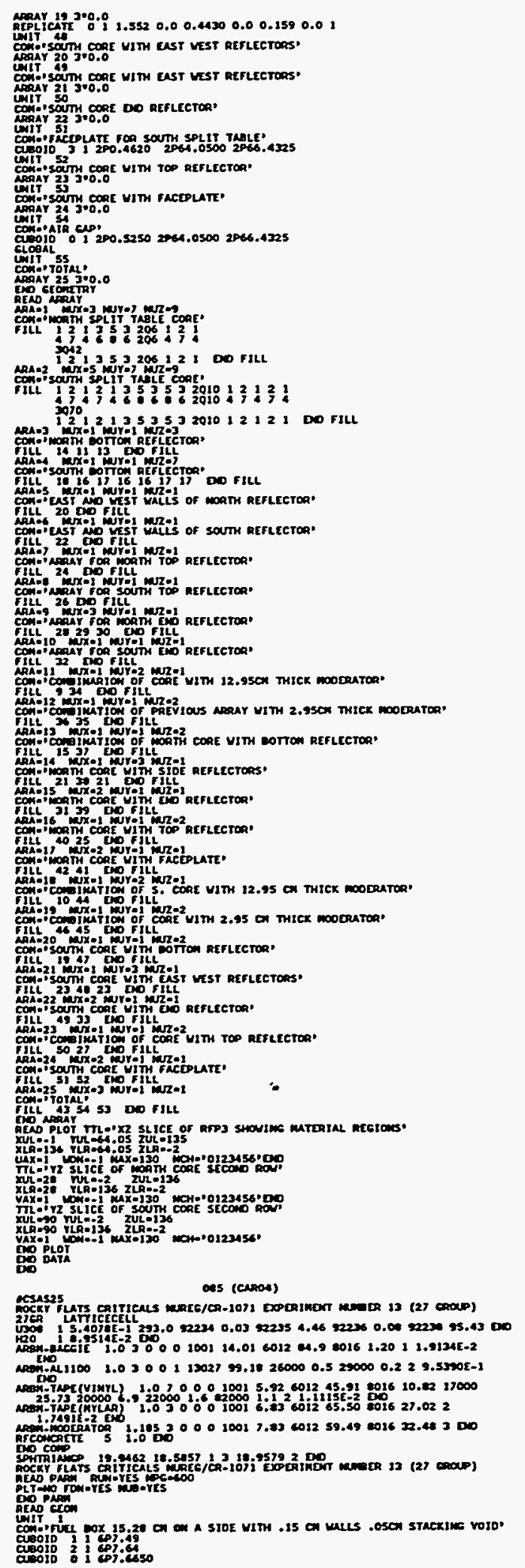

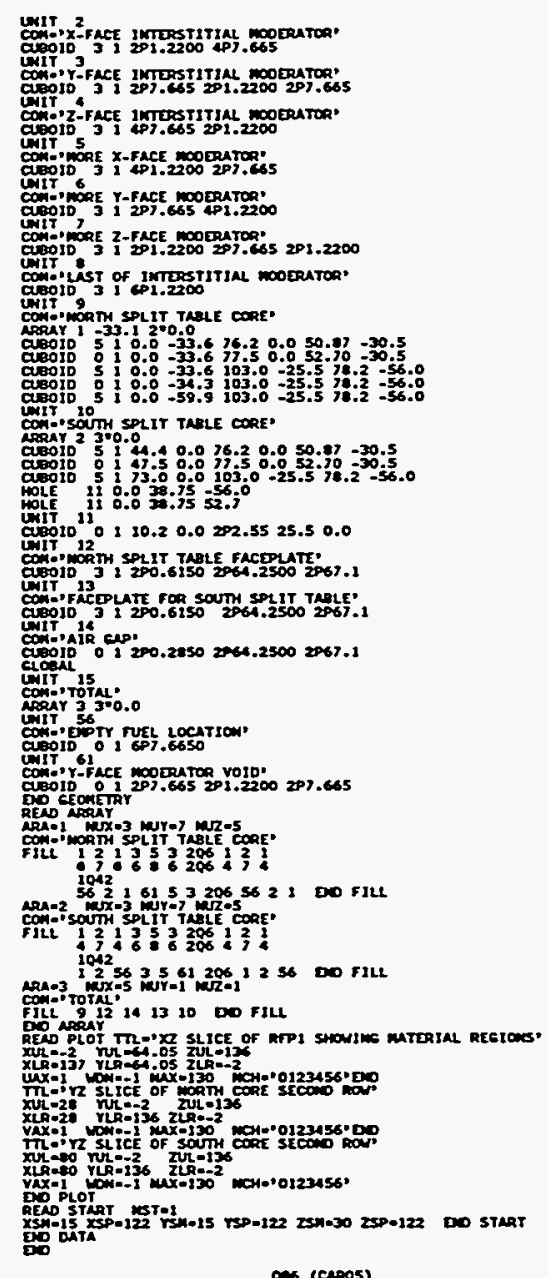

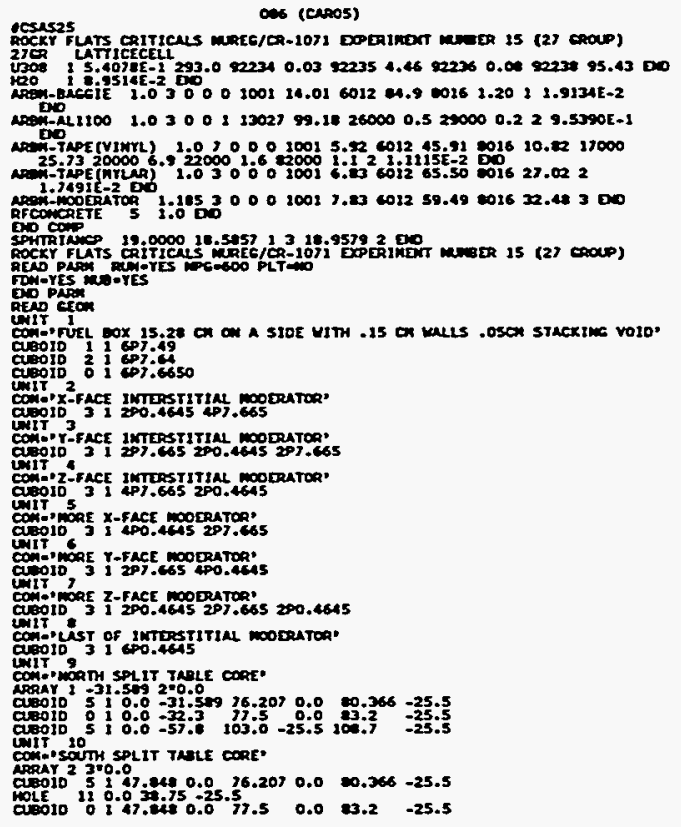



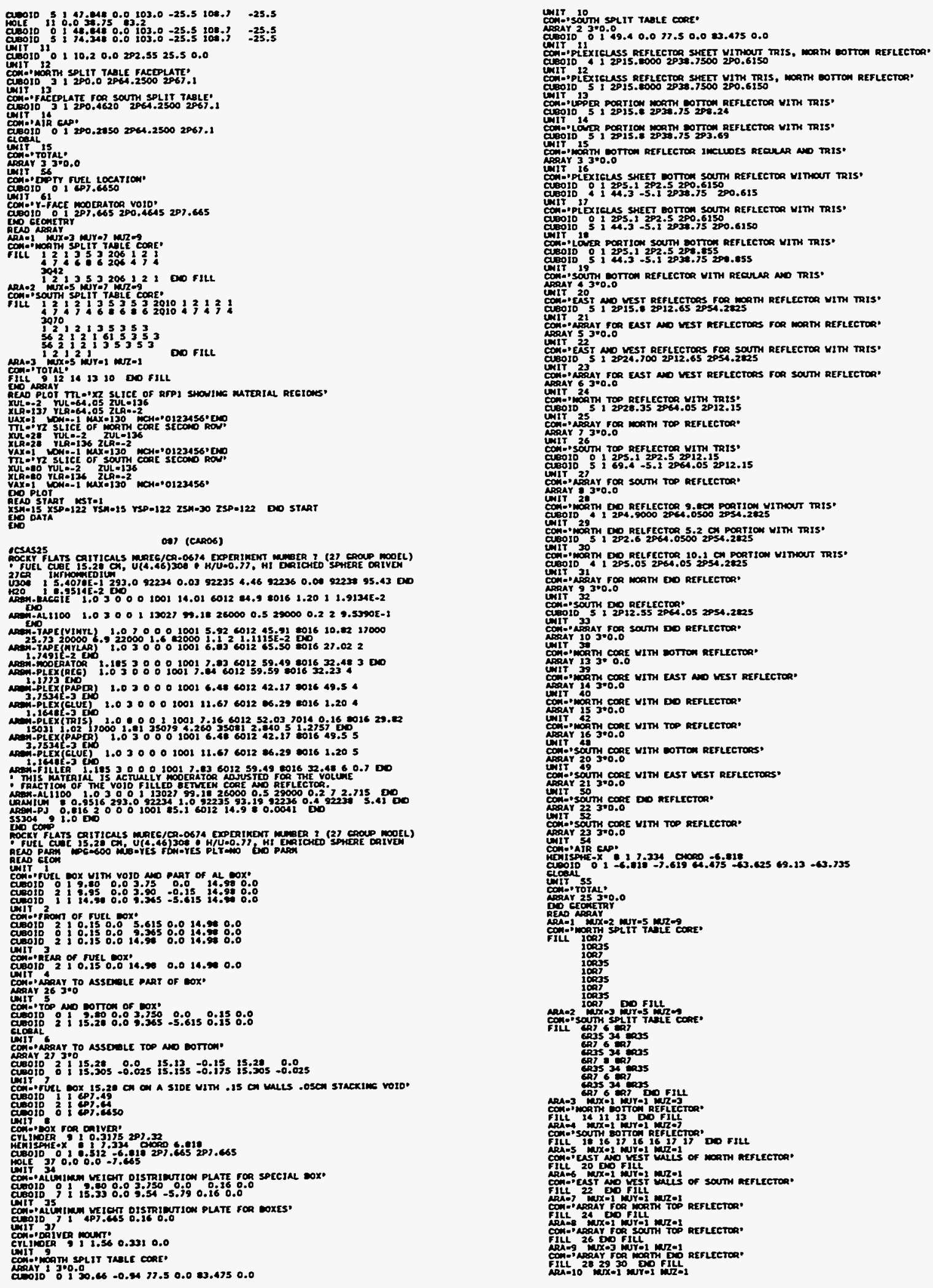


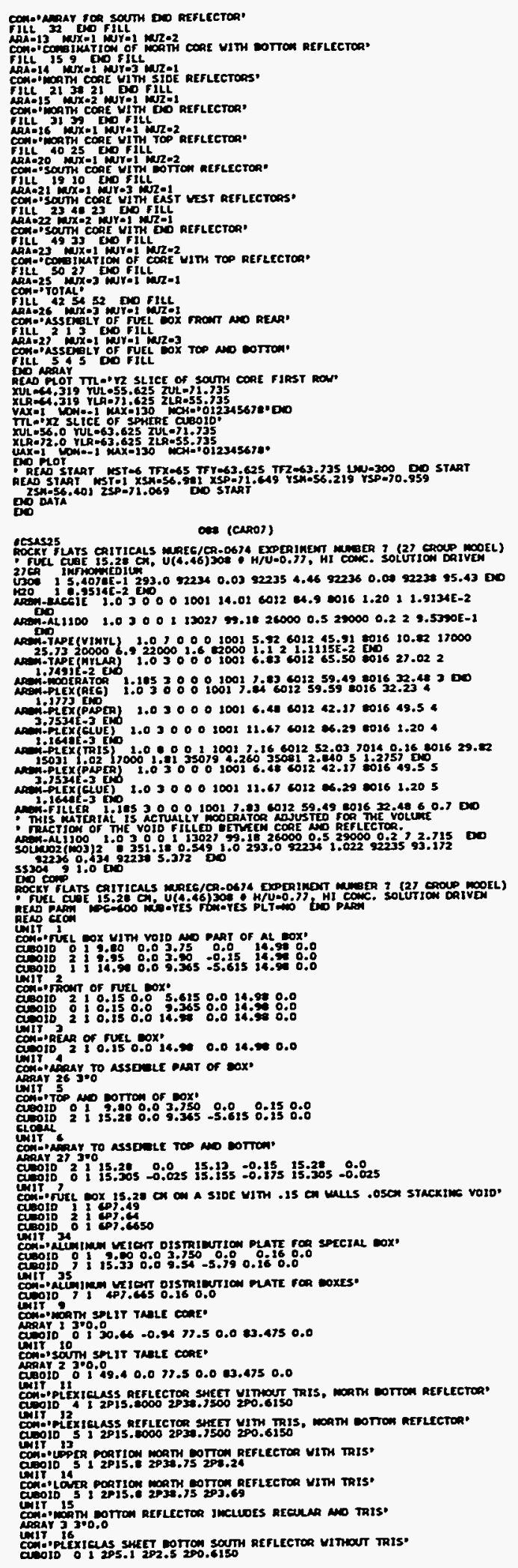

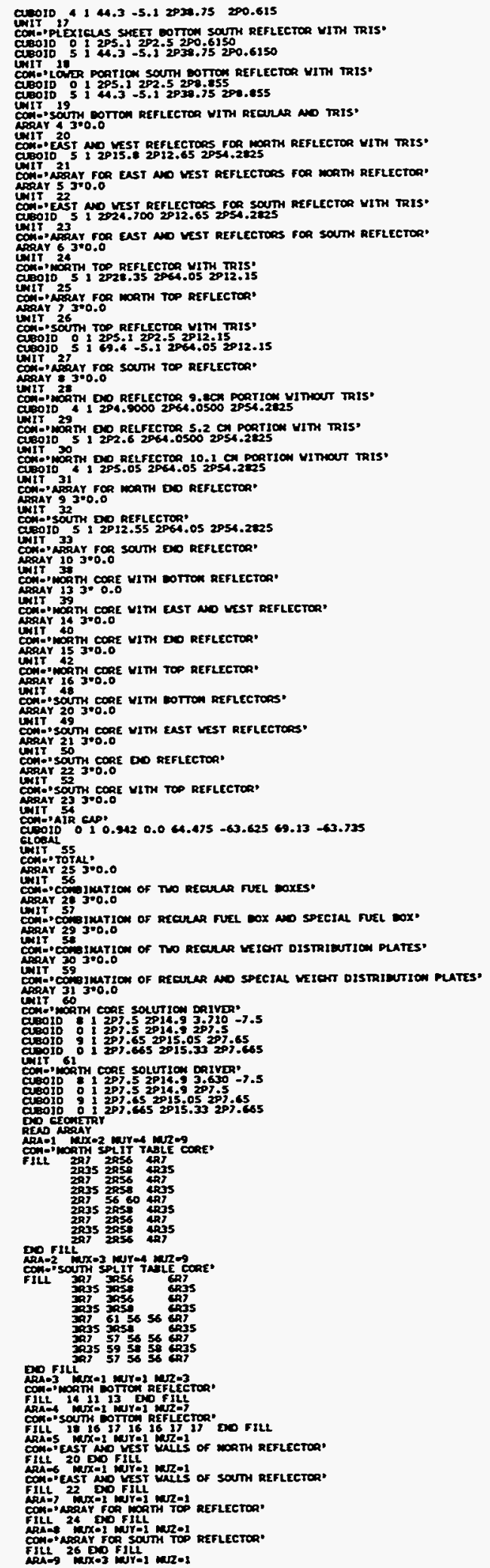




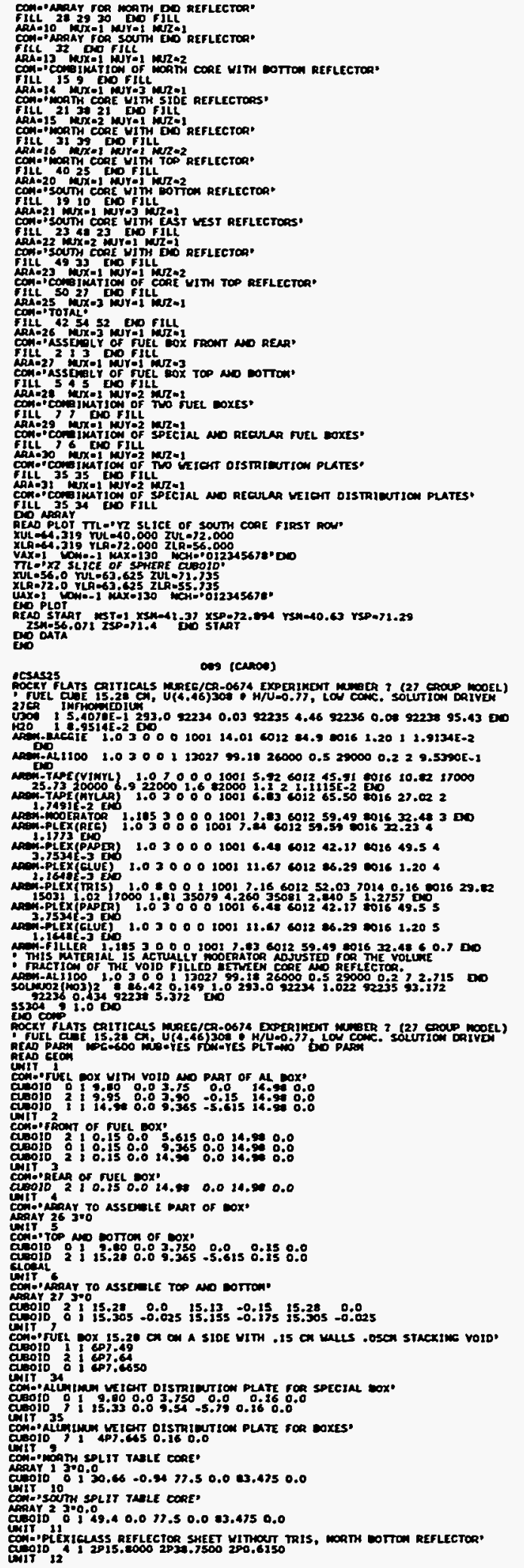

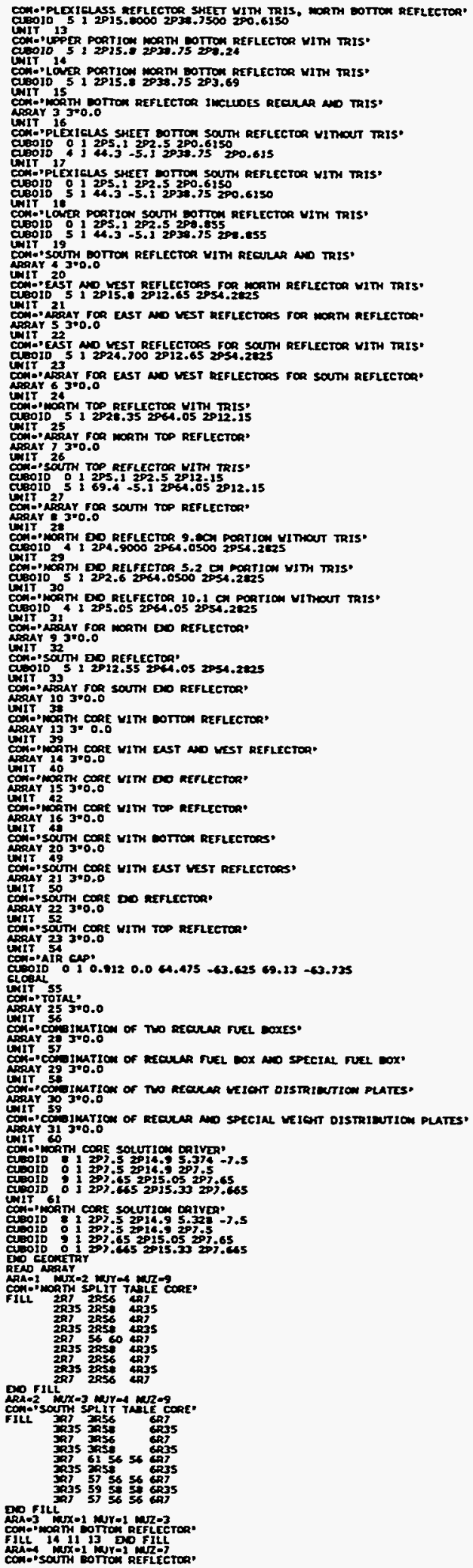




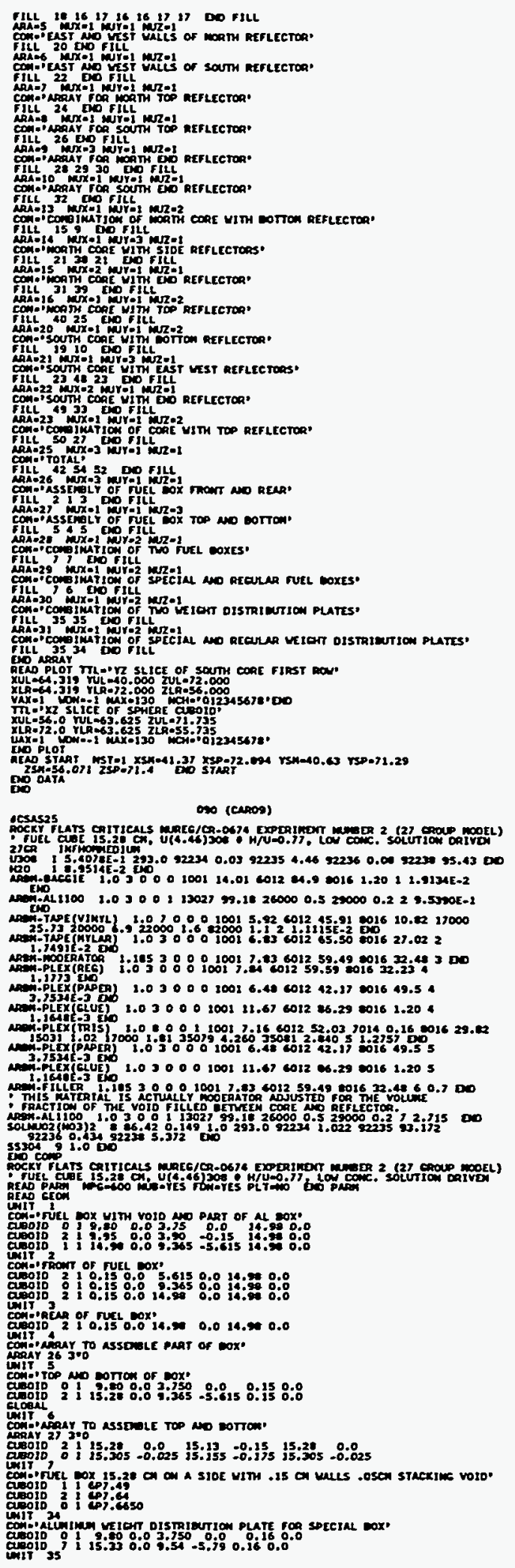

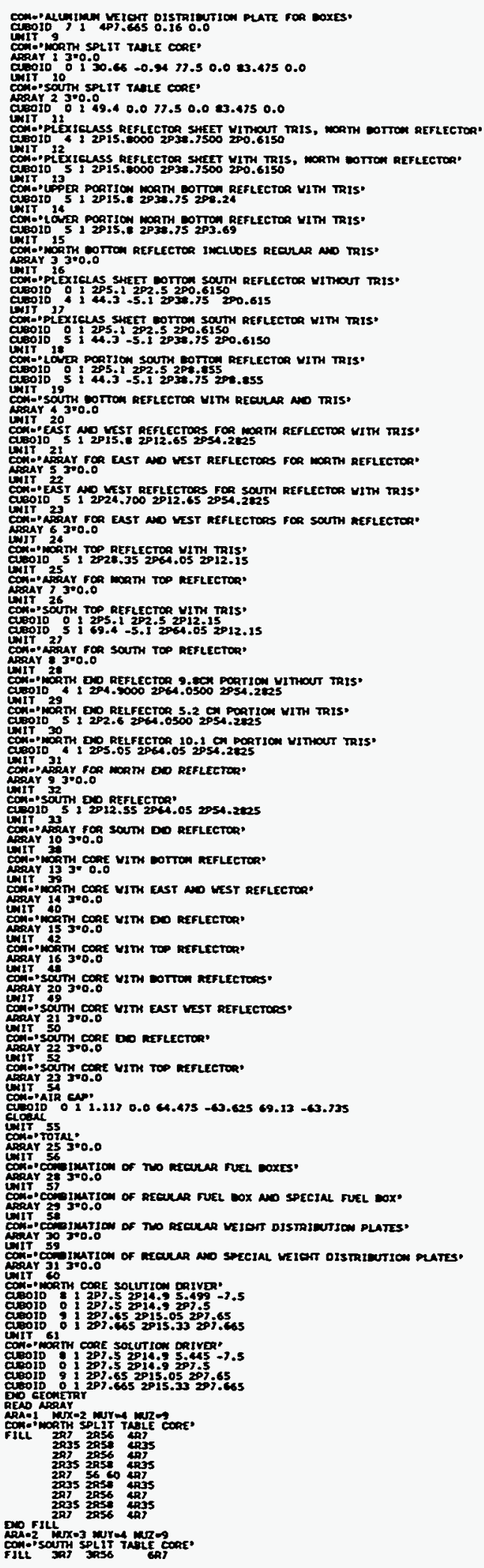




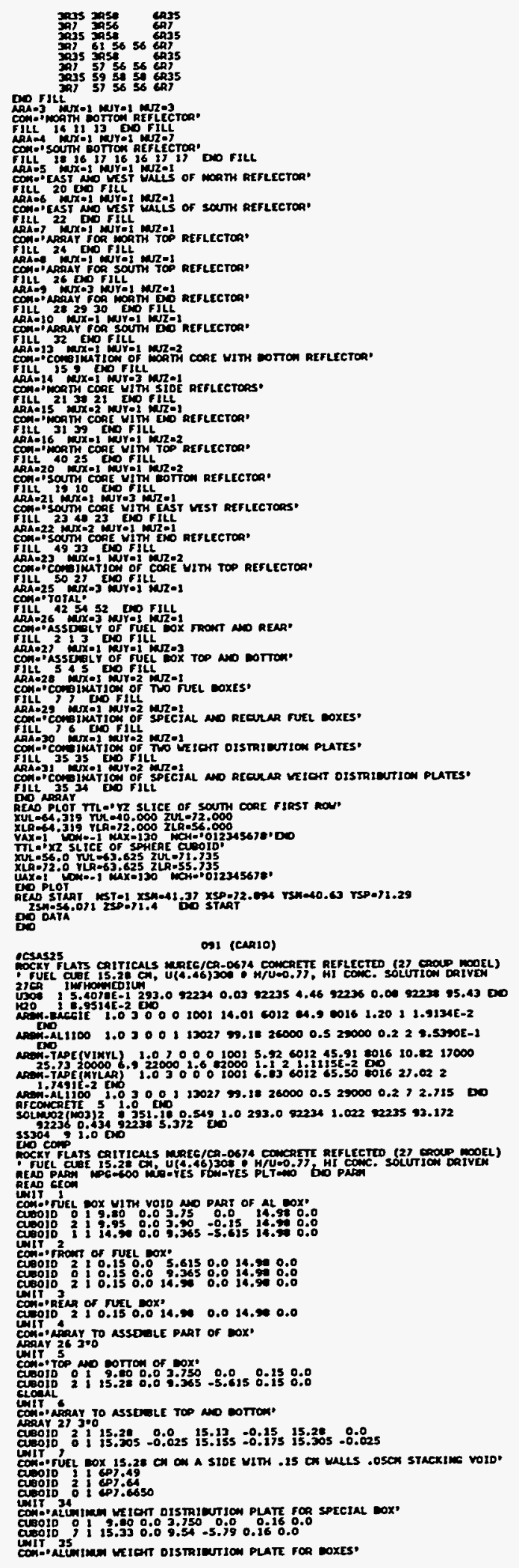

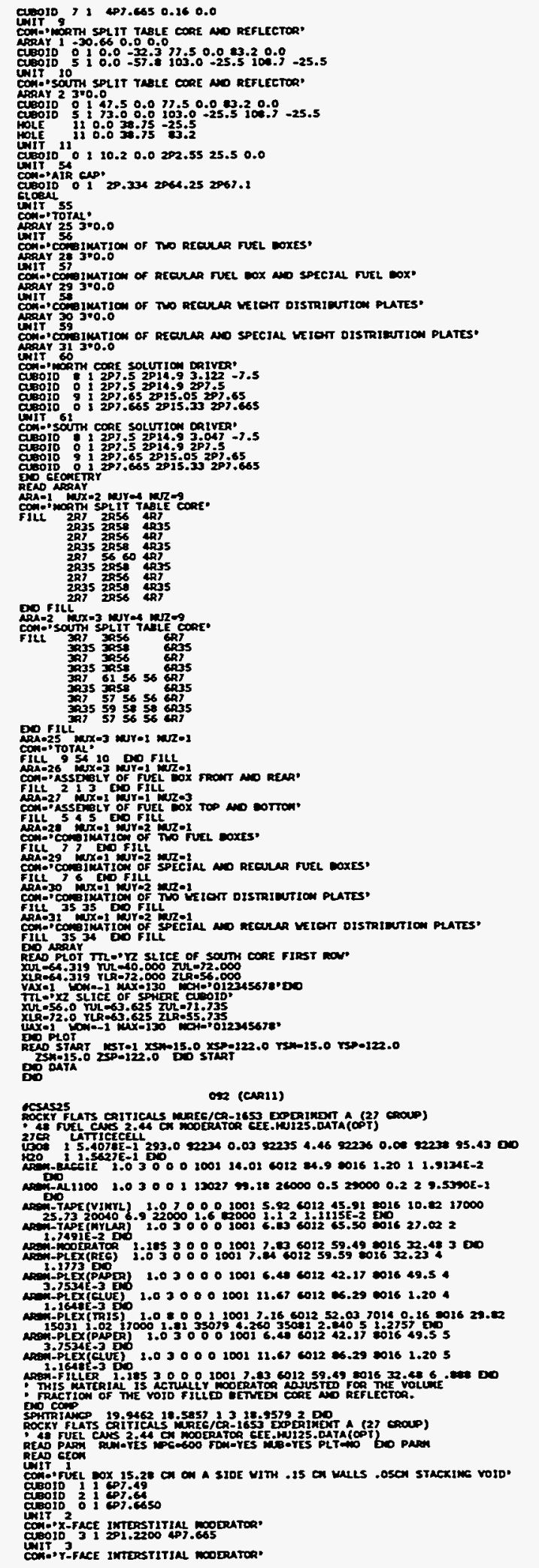




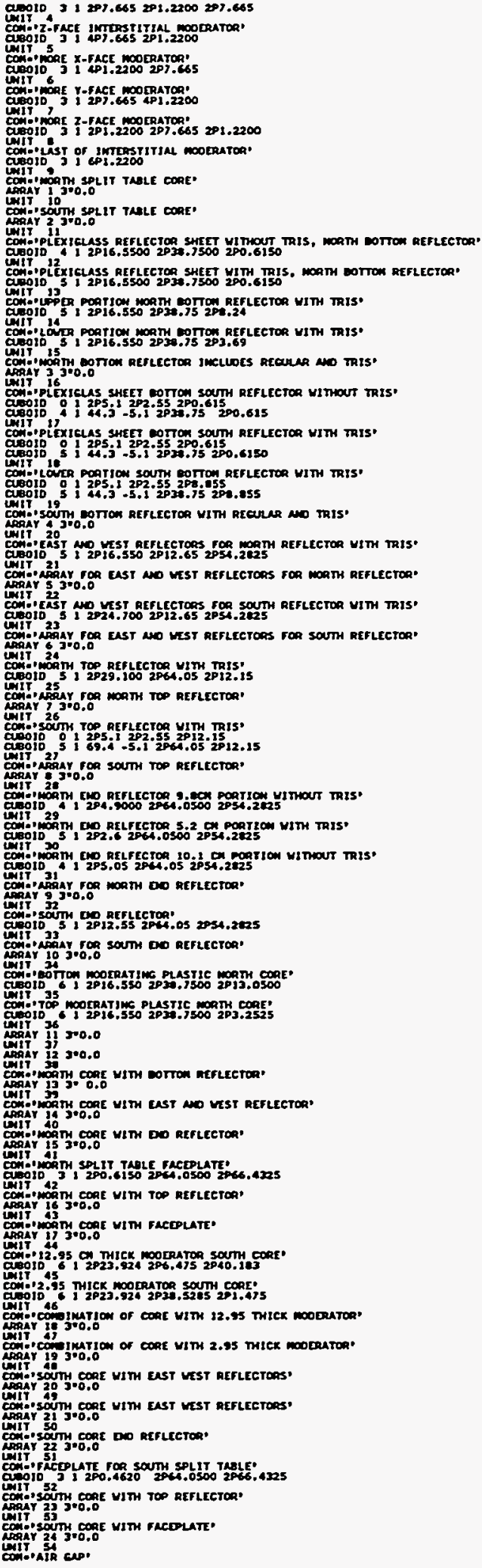

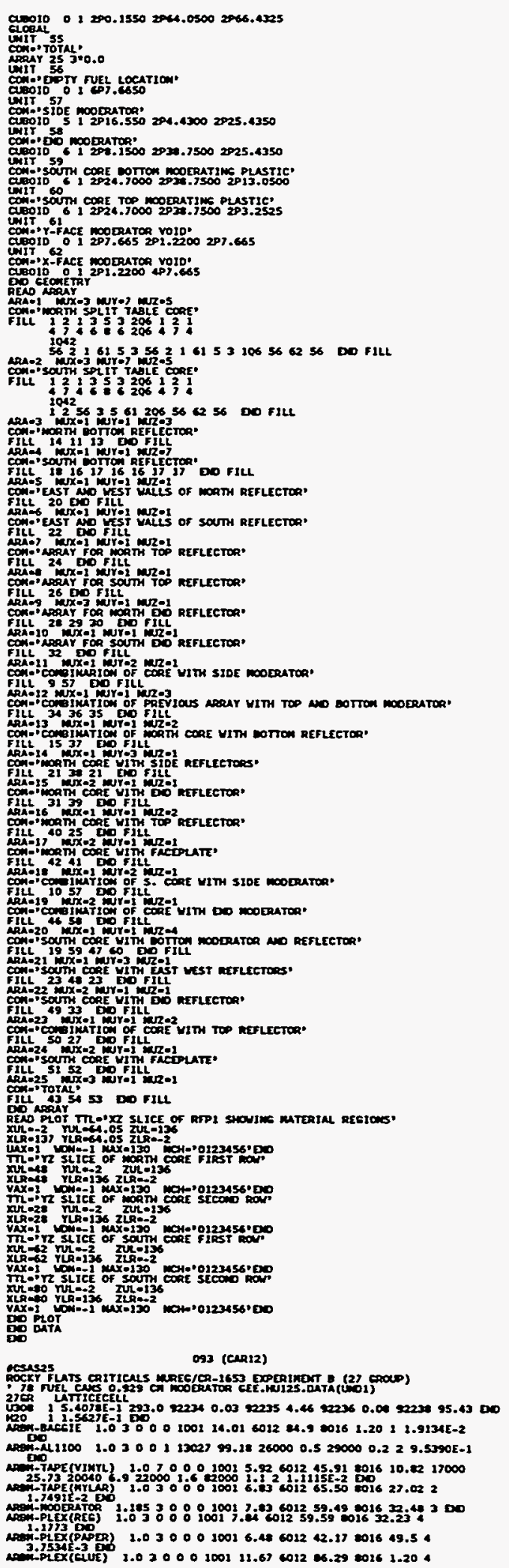




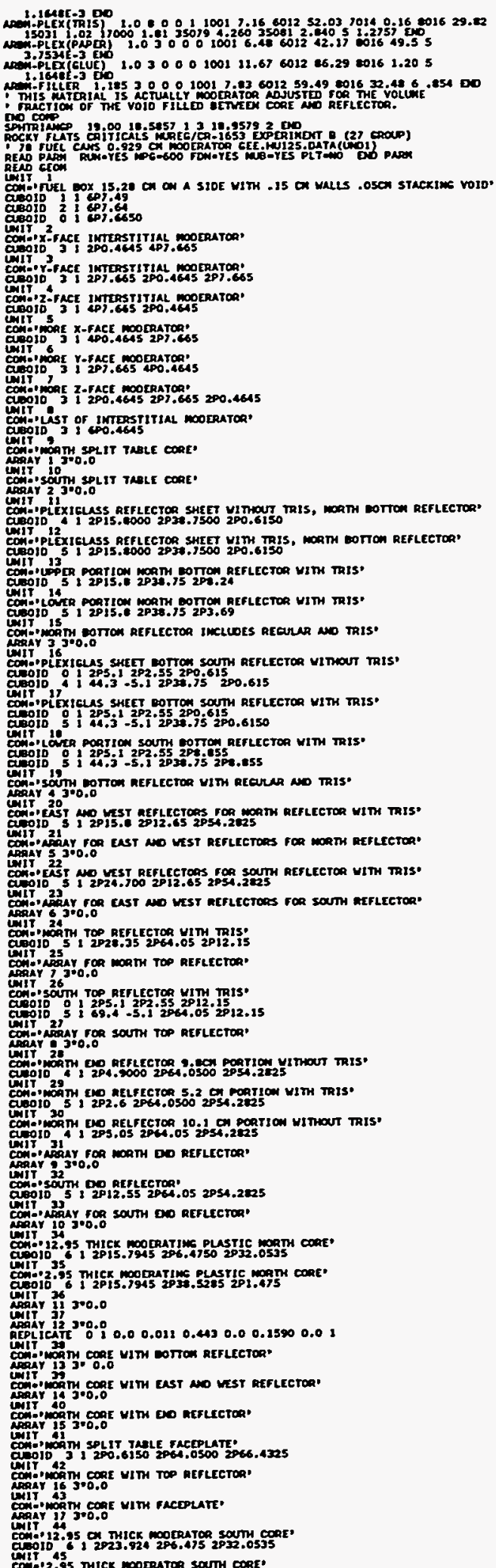

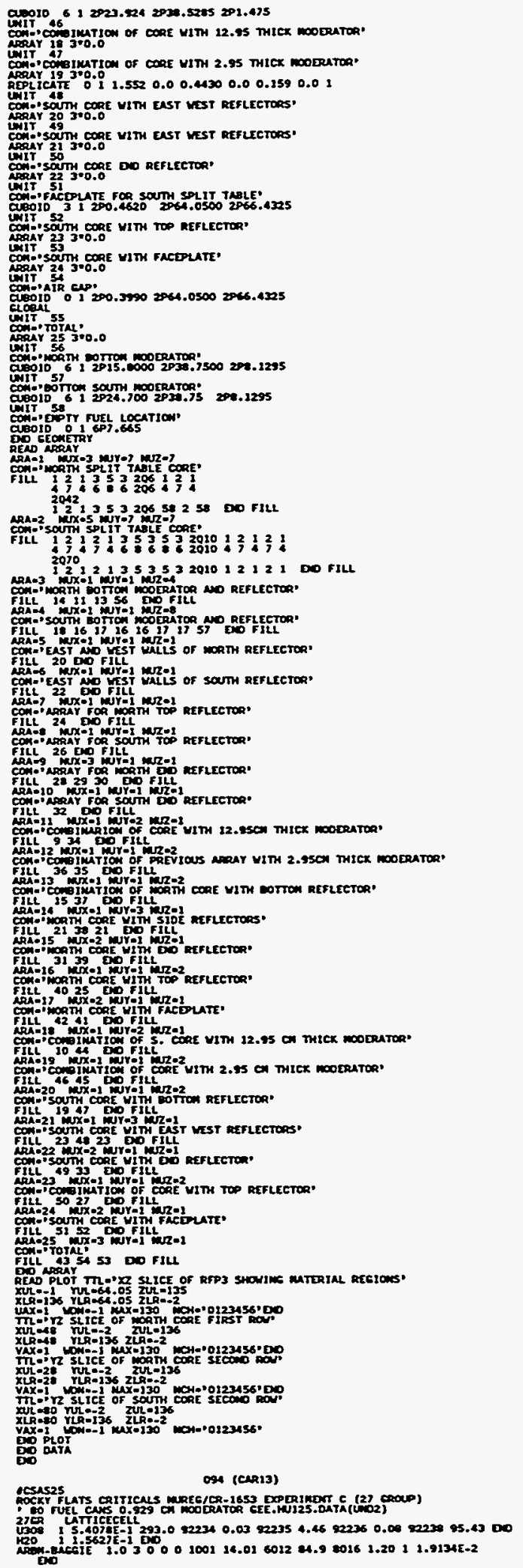




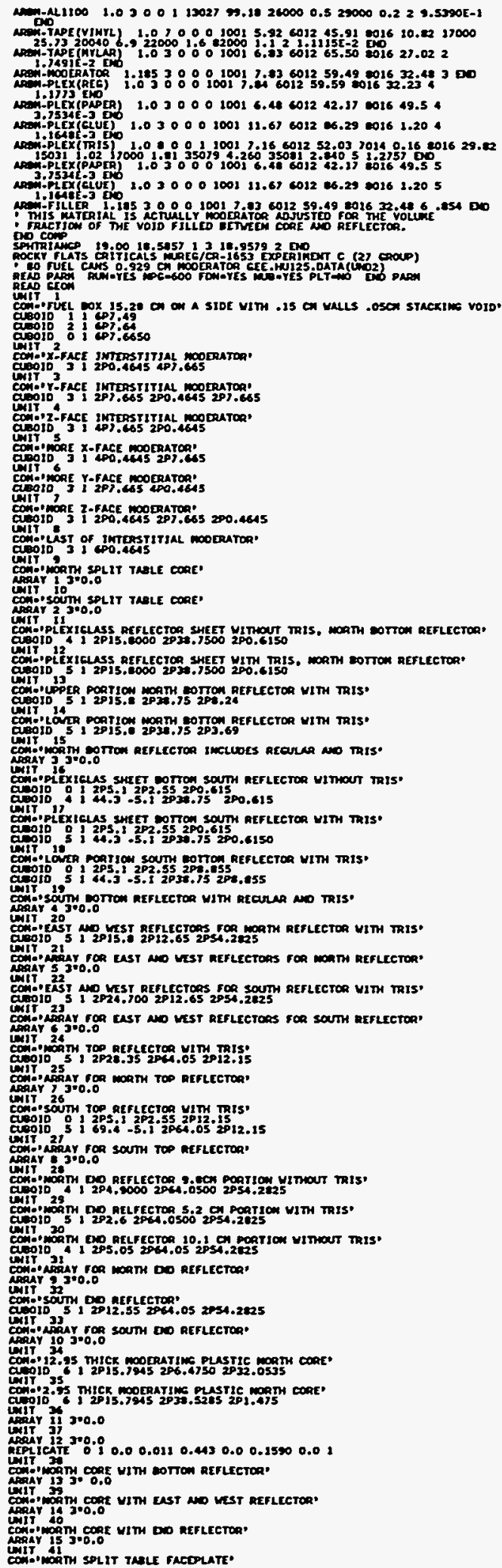

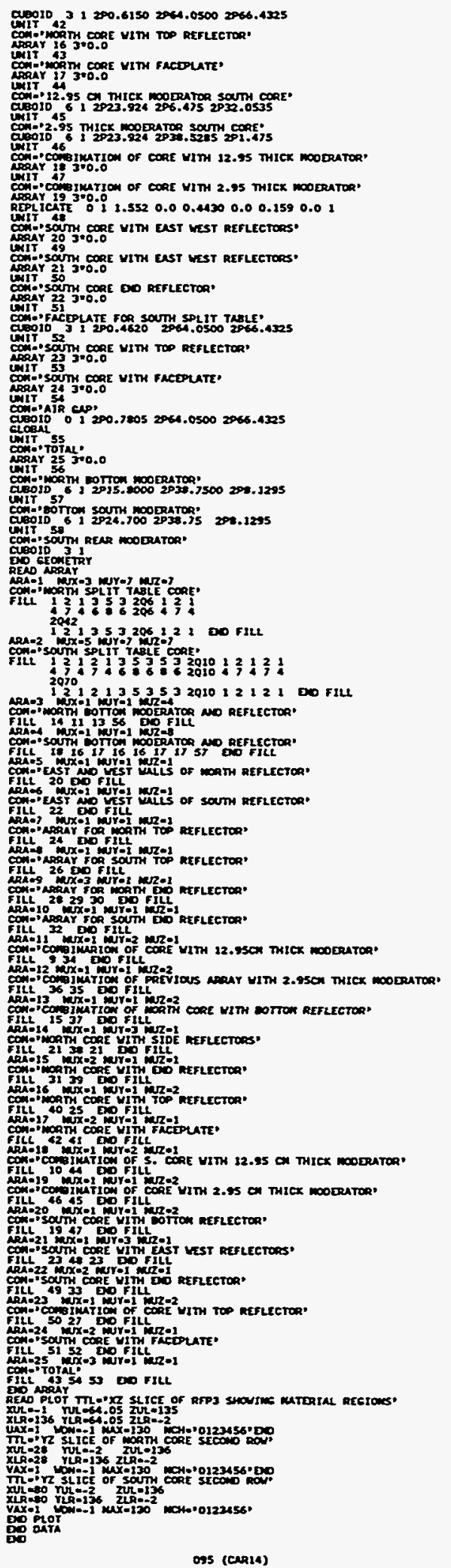




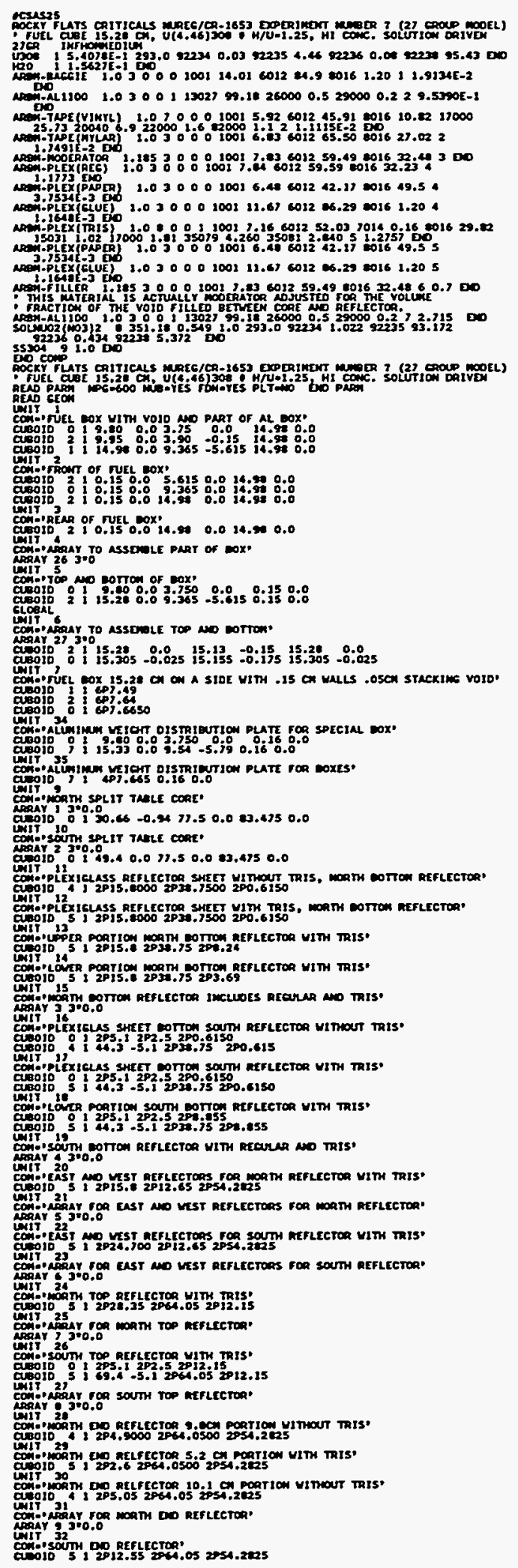

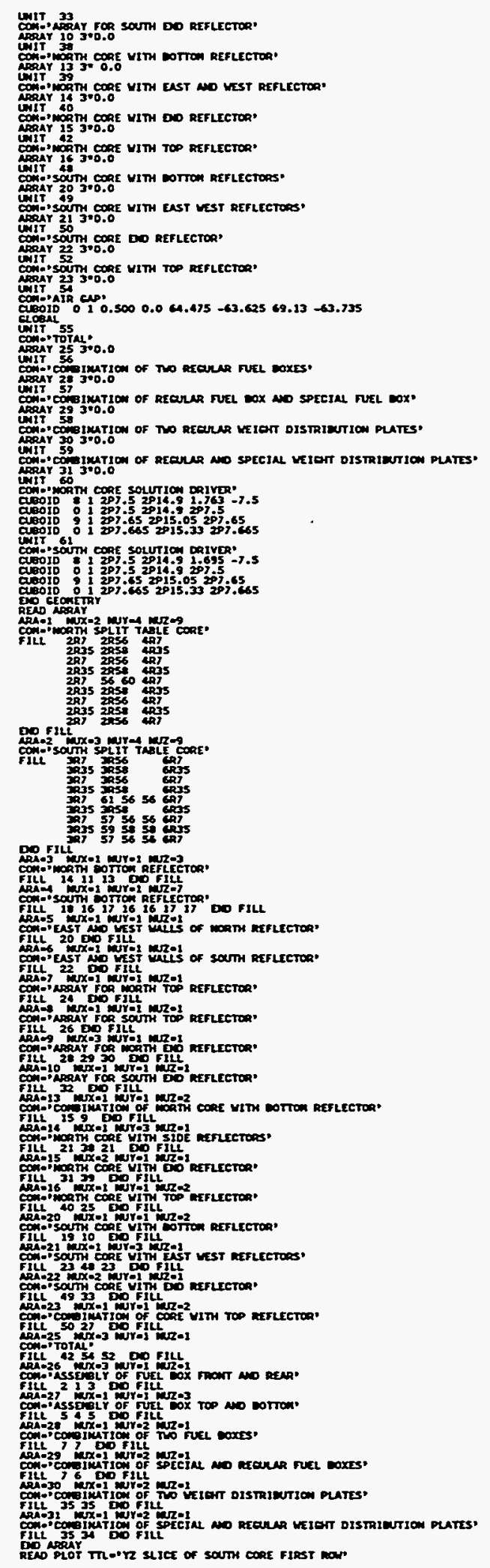




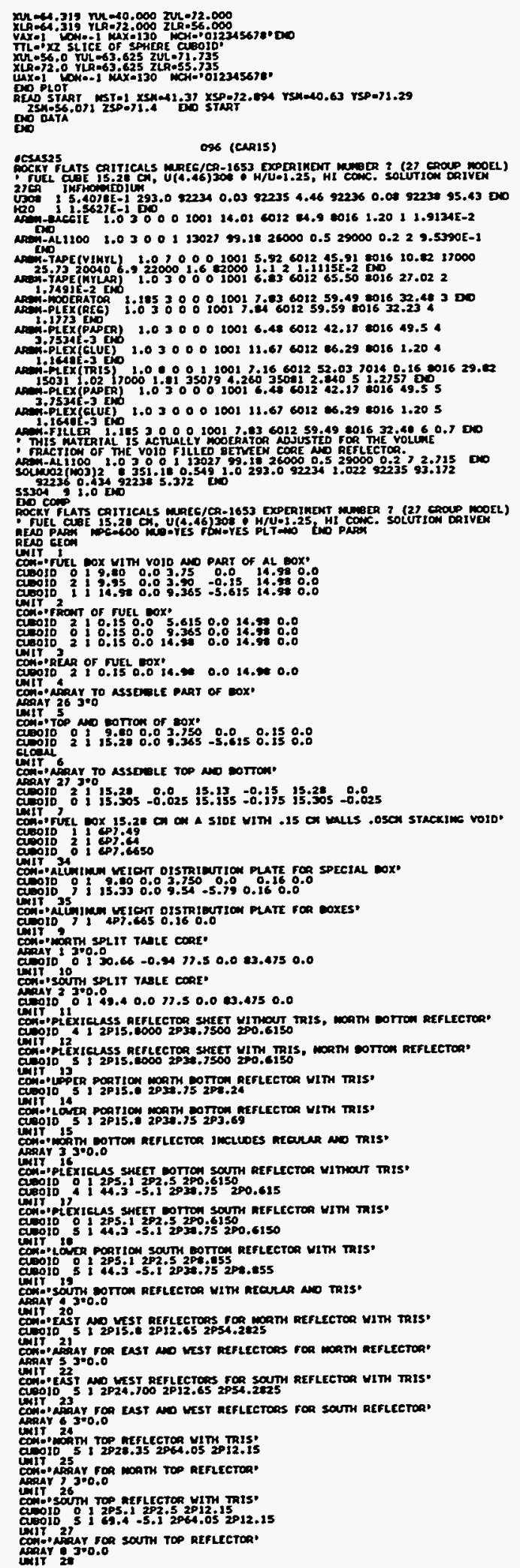

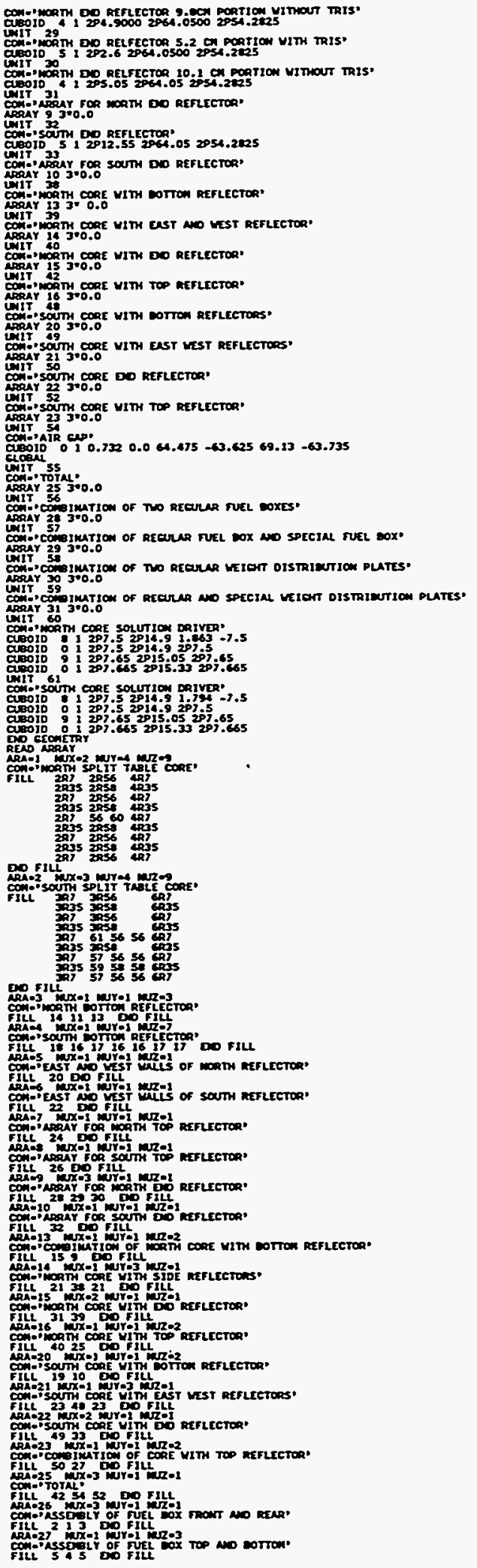




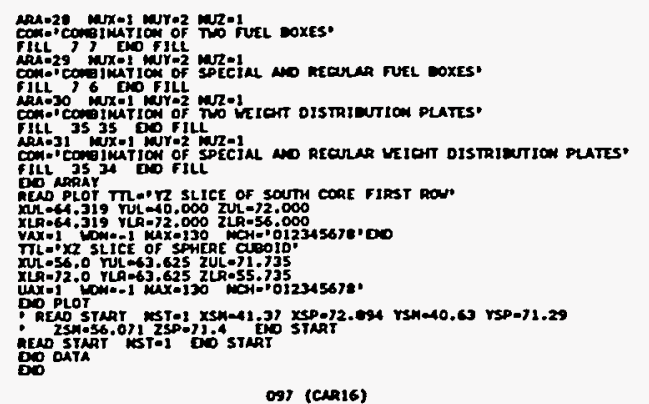

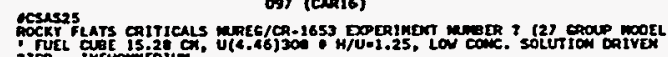
270 作D

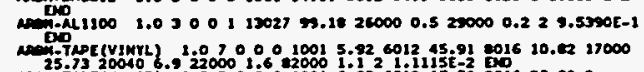

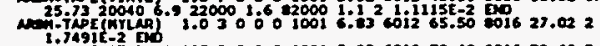

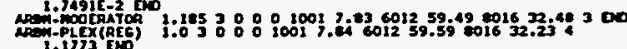

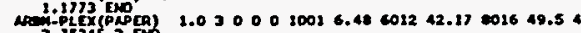
M.7.753

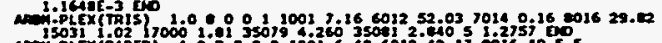
Nemptex

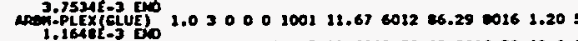

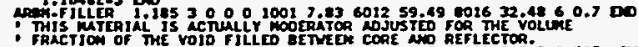

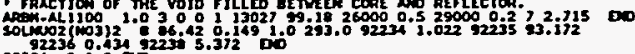
5504001000

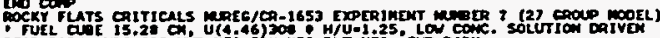
and

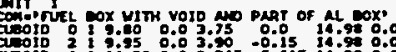

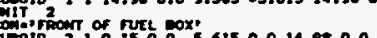

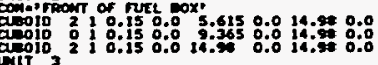

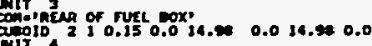

conirivary

Cal1

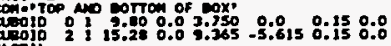

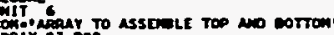

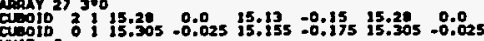

contr.

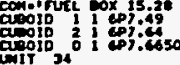

Qmin

(1)

Cons

Colf

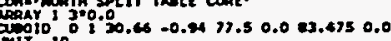

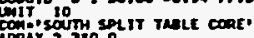

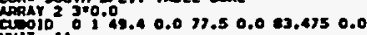

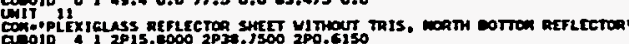

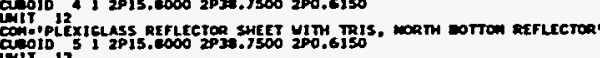

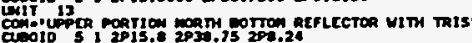

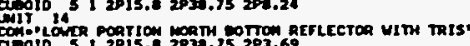

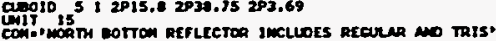

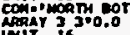

(1)

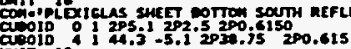

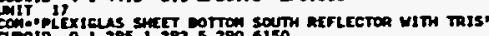

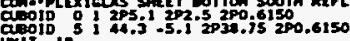

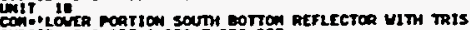

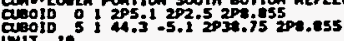

W

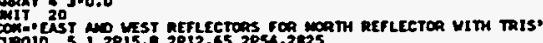

WIIT 21 is

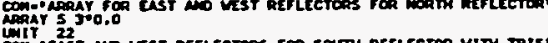

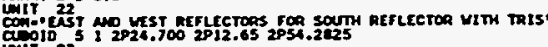

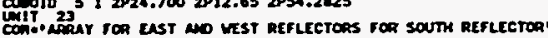

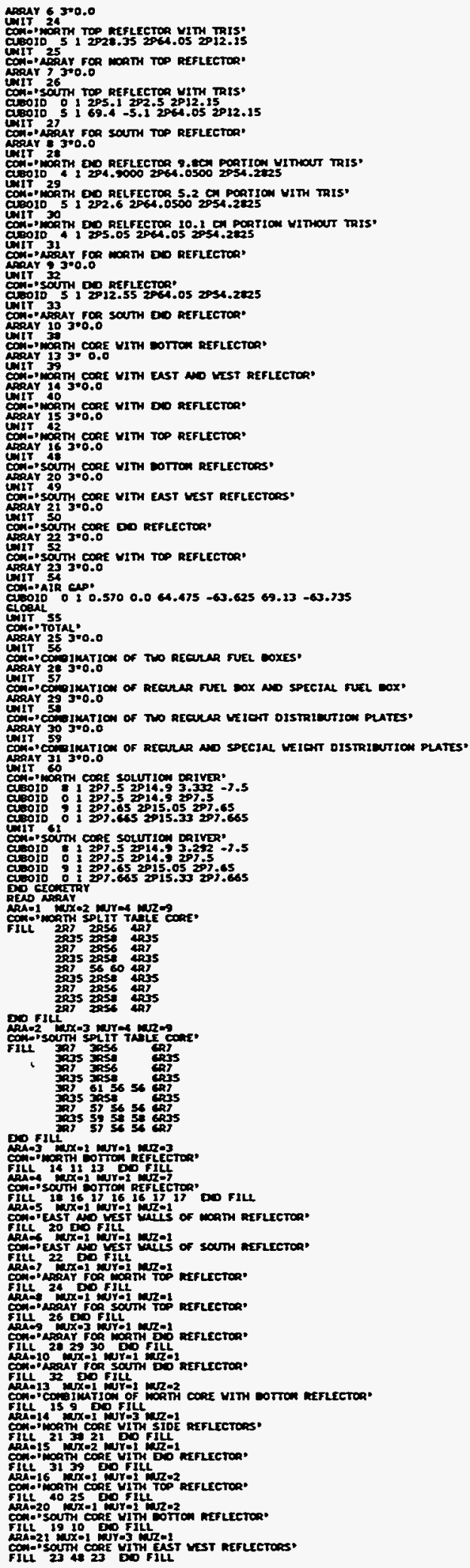




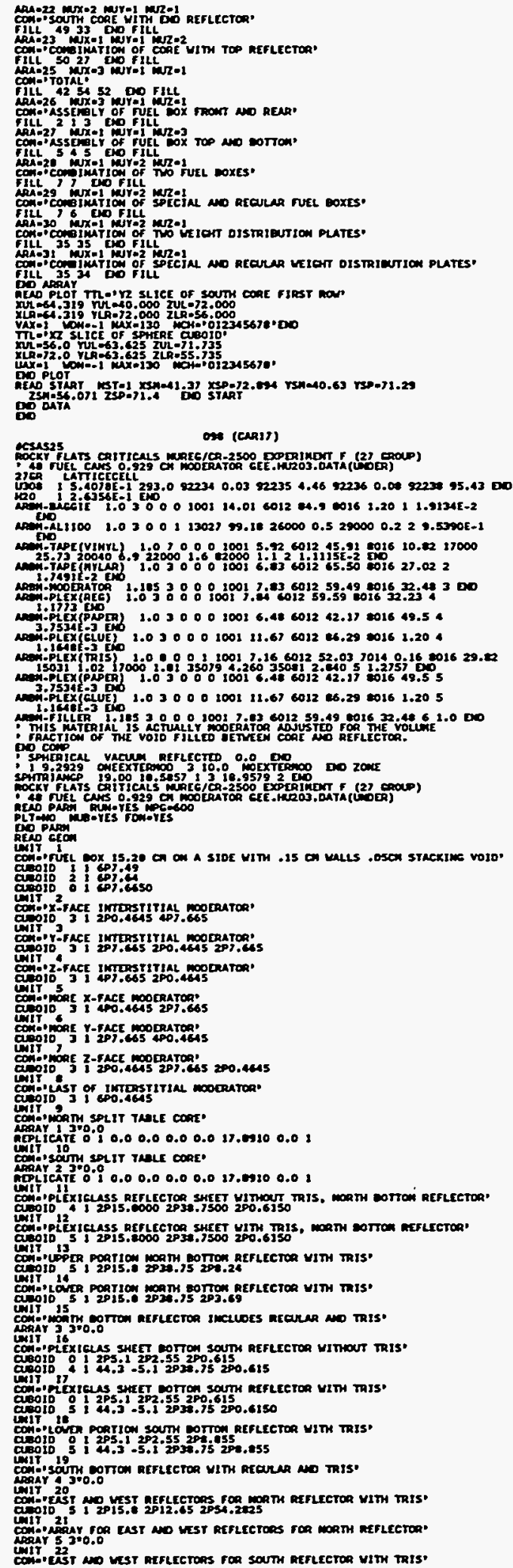

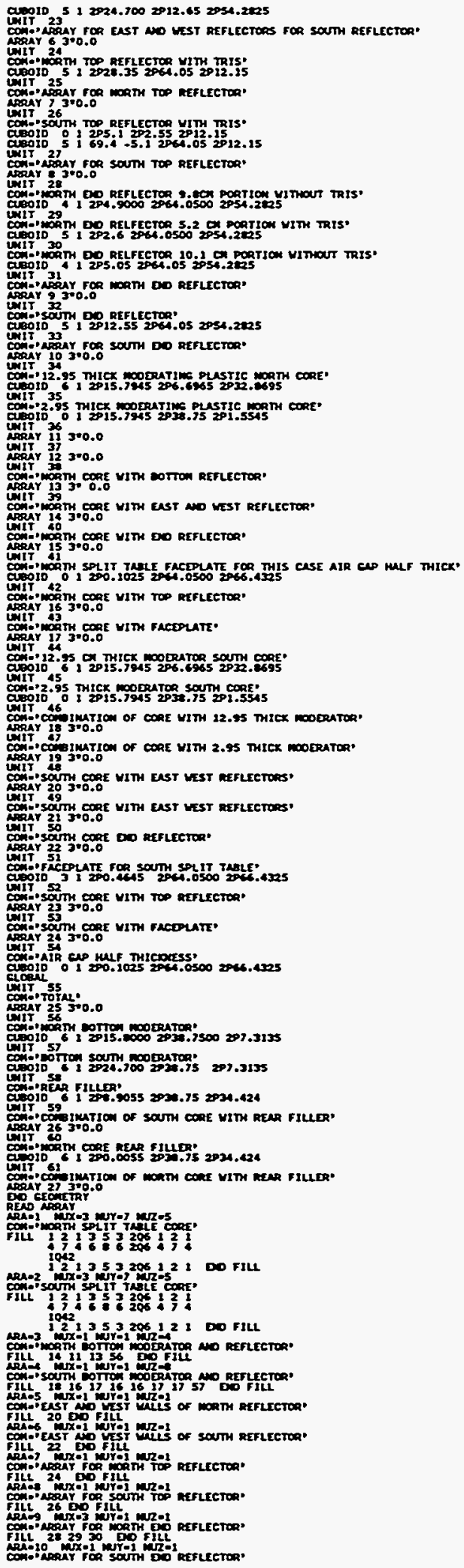




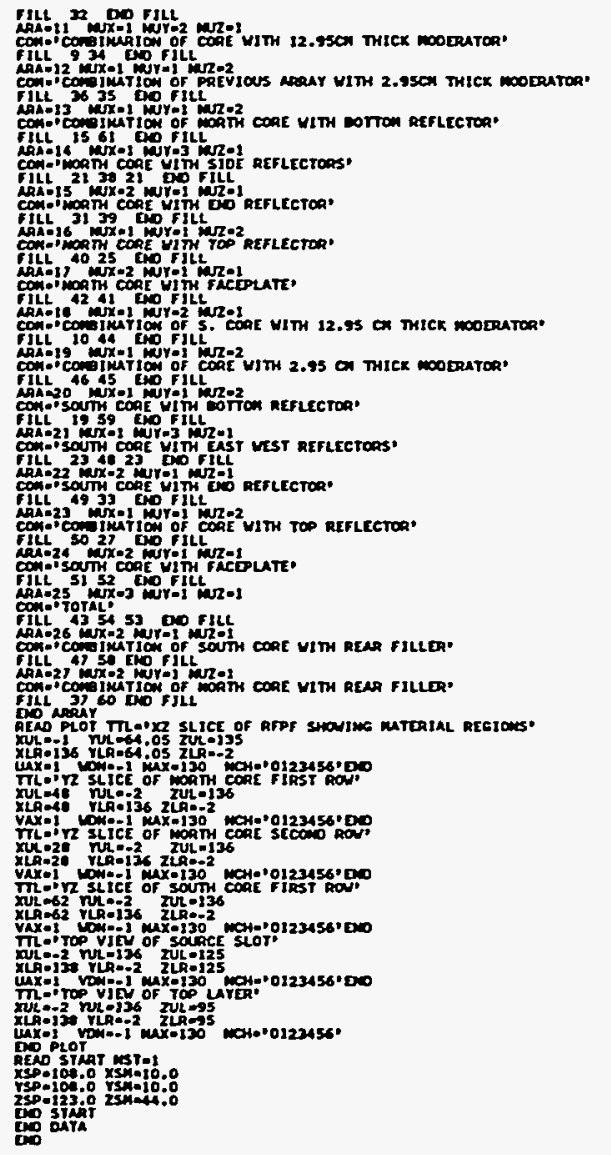

on (certo)

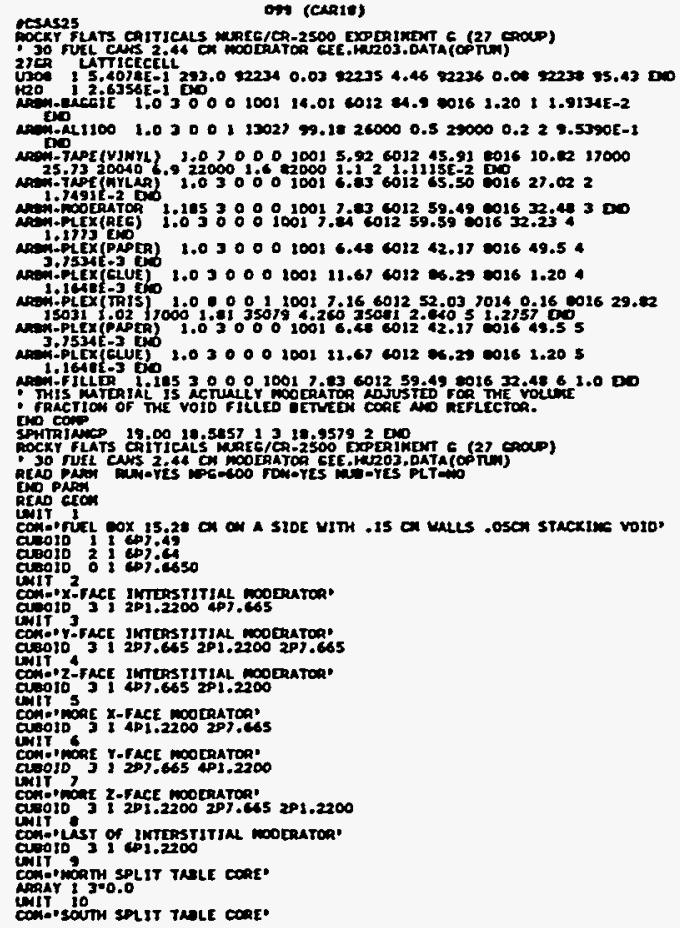

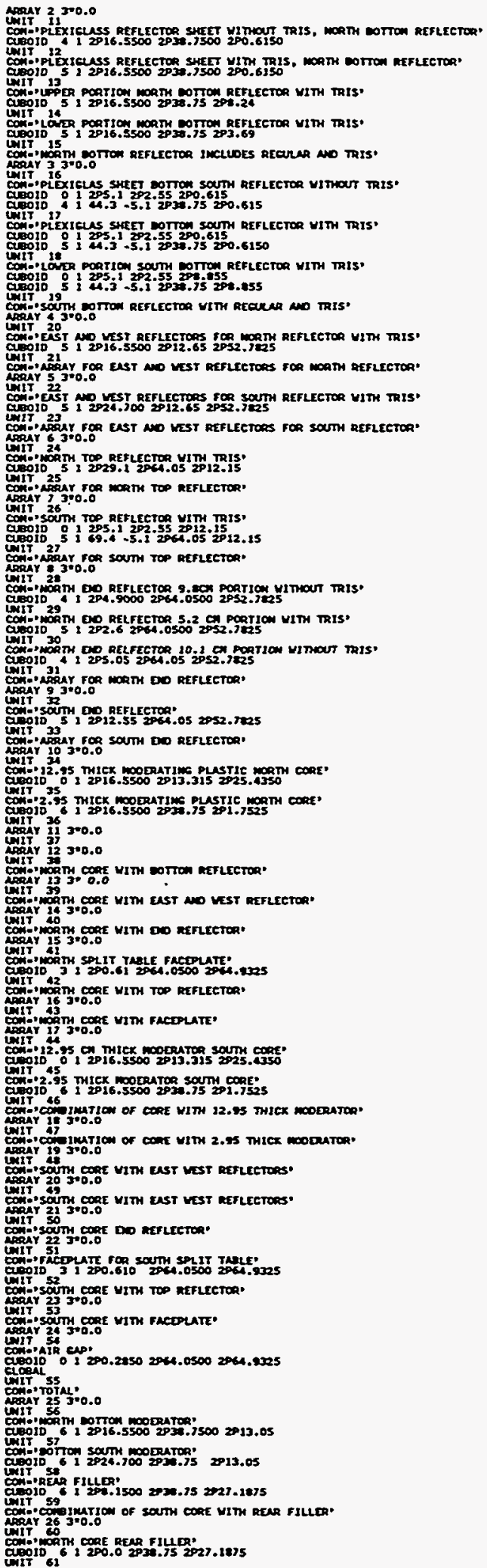




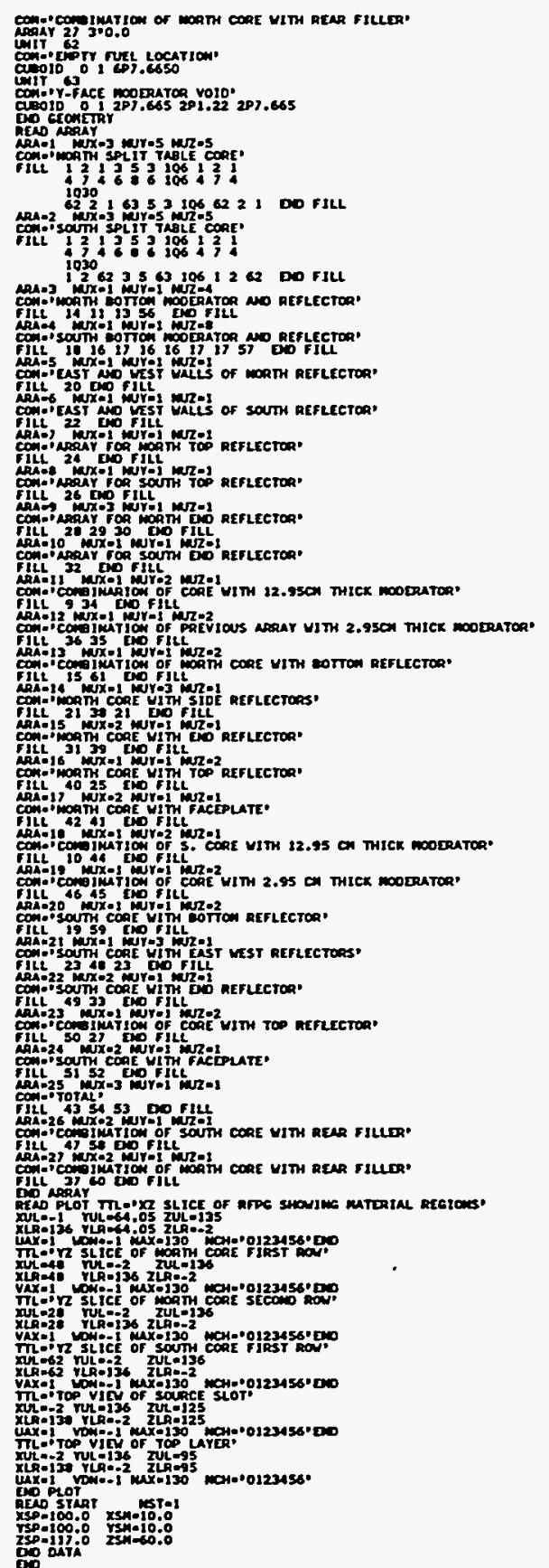

100 (CNA19)

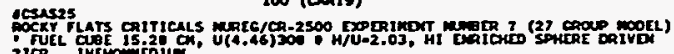

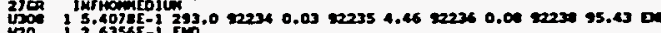

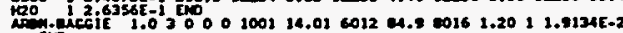

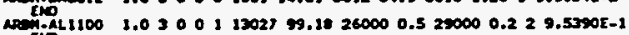

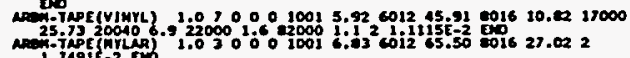
(1)

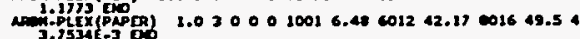
aven:-pLEXILLVE) 1.03000100111 .67001206 .2400161 .204

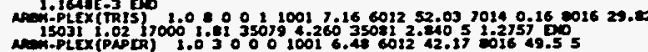

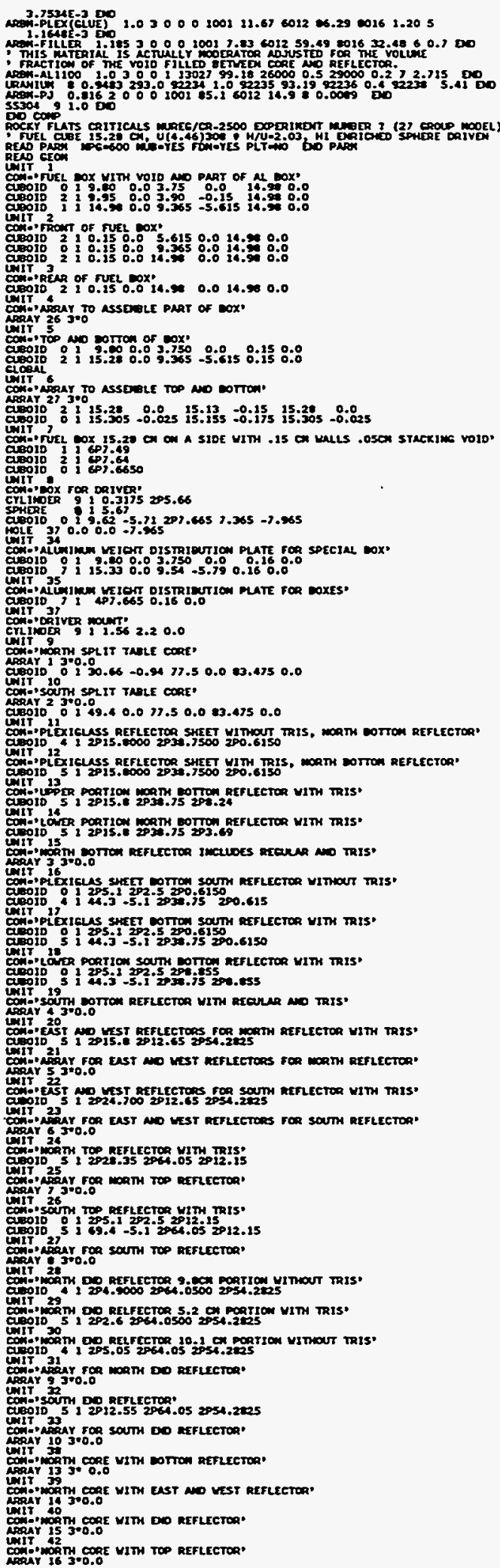

E-34 


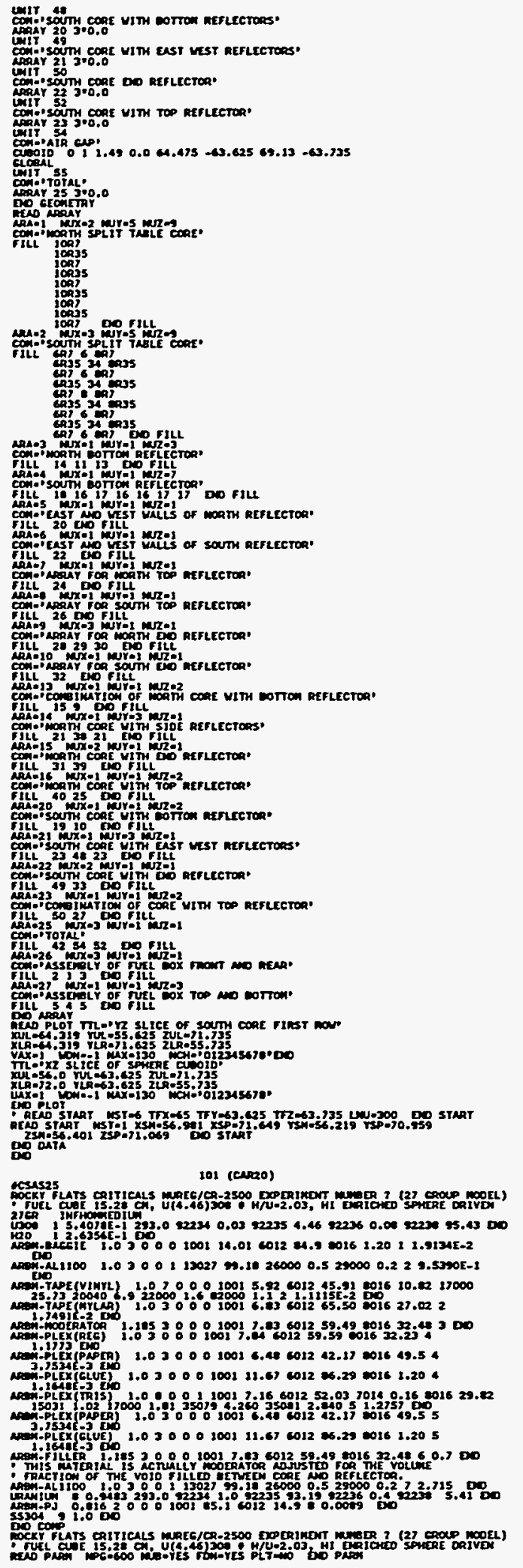

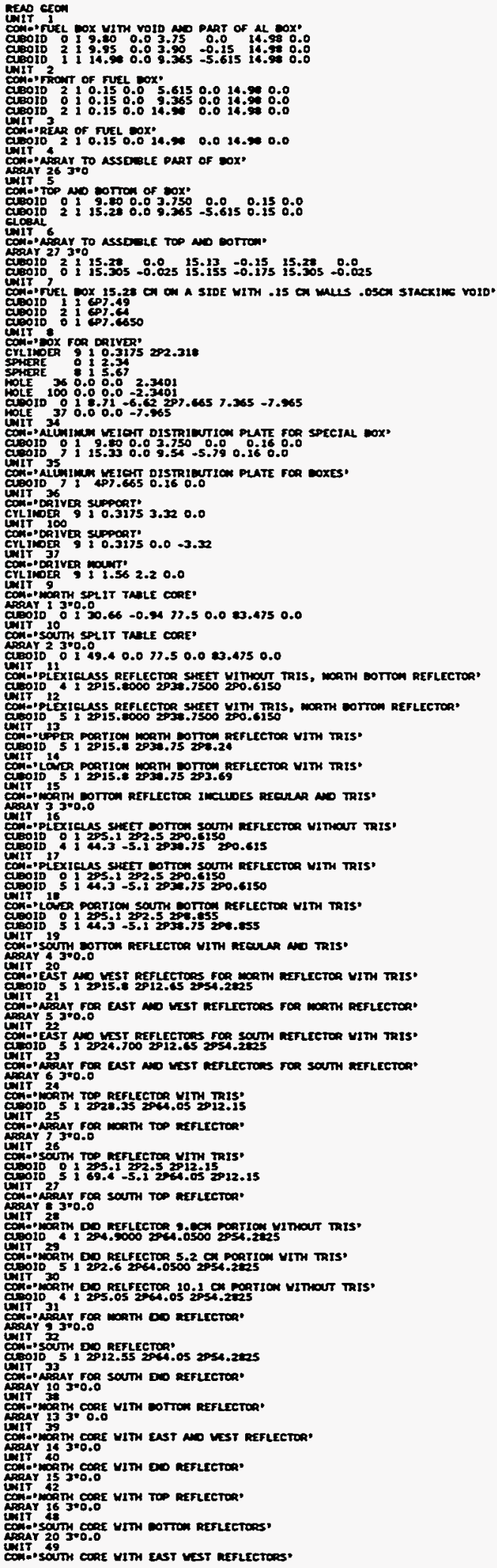




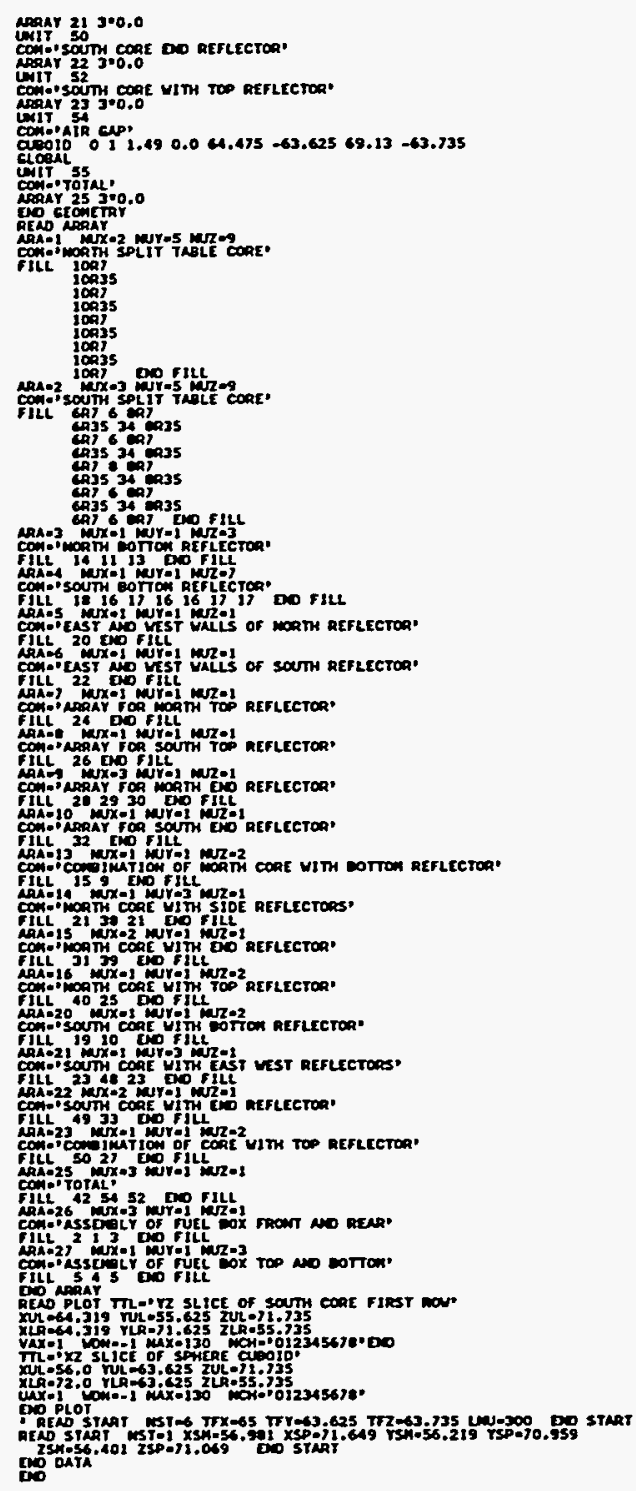




\section{APPENDIX E4: TABLE 4 INPUTS}

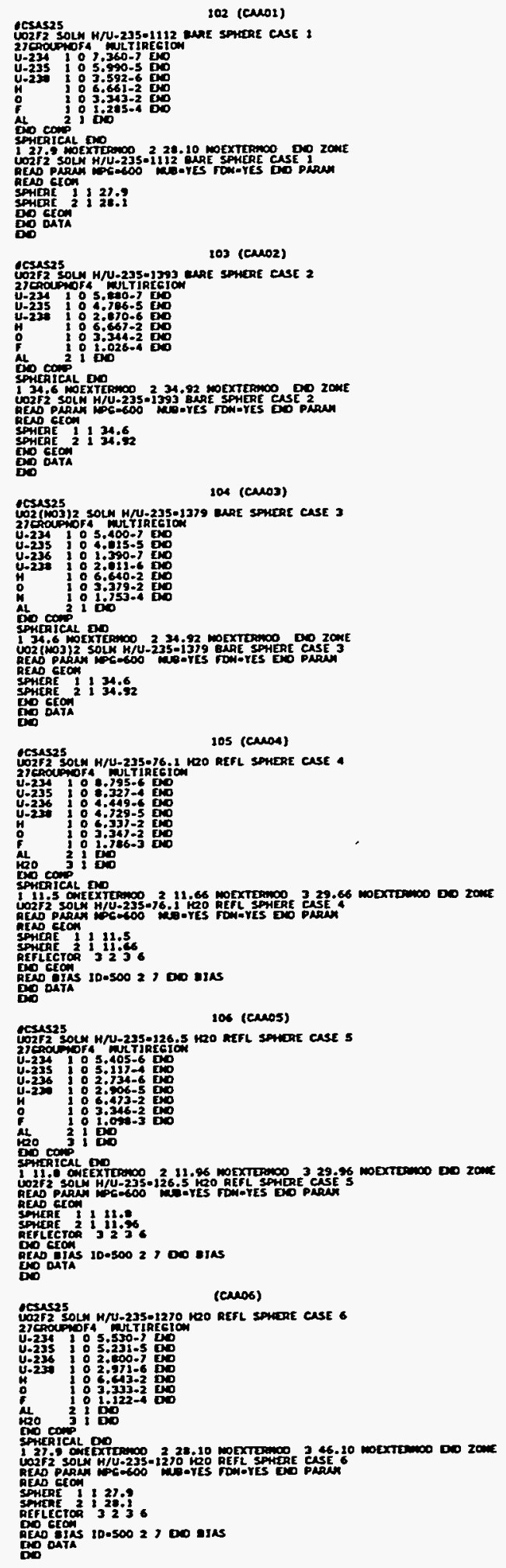

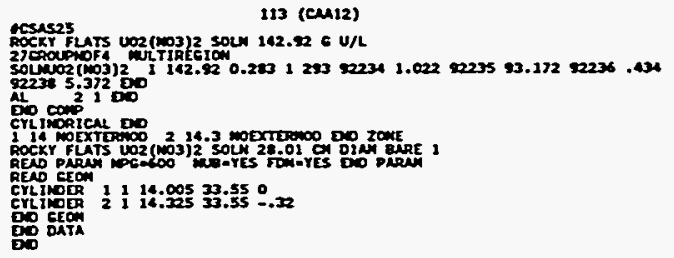

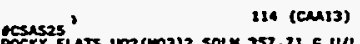

210

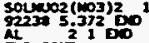

Drophical op

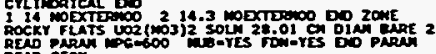

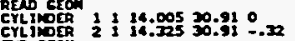

被

115 (cu14)
cosesses

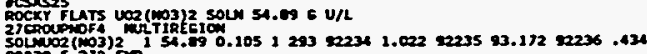
22.310 5.312200

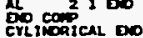

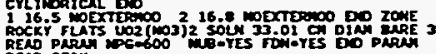

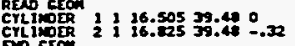

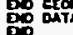

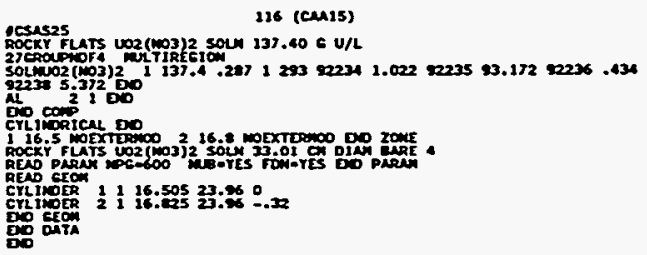

327 (censes)

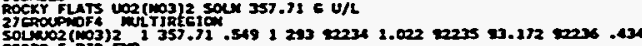
423153200

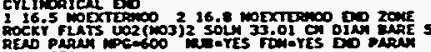

cecting

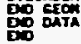
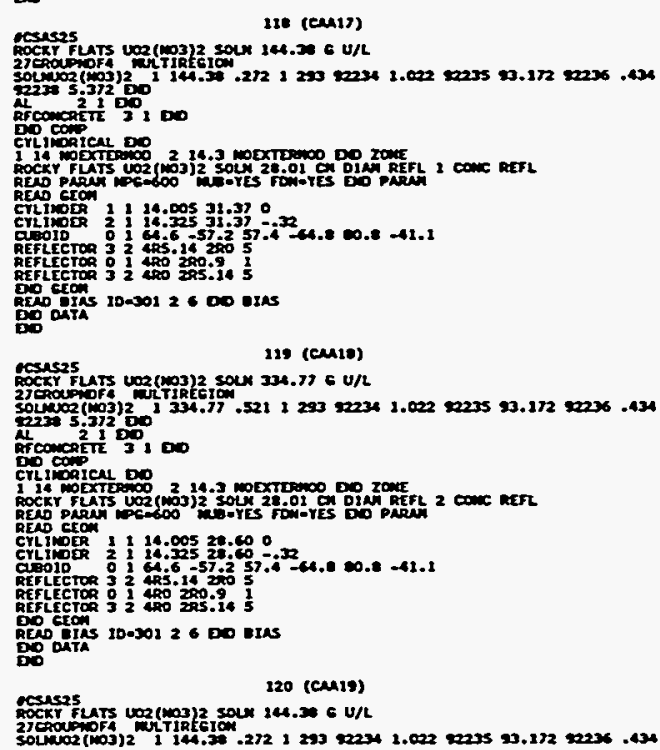


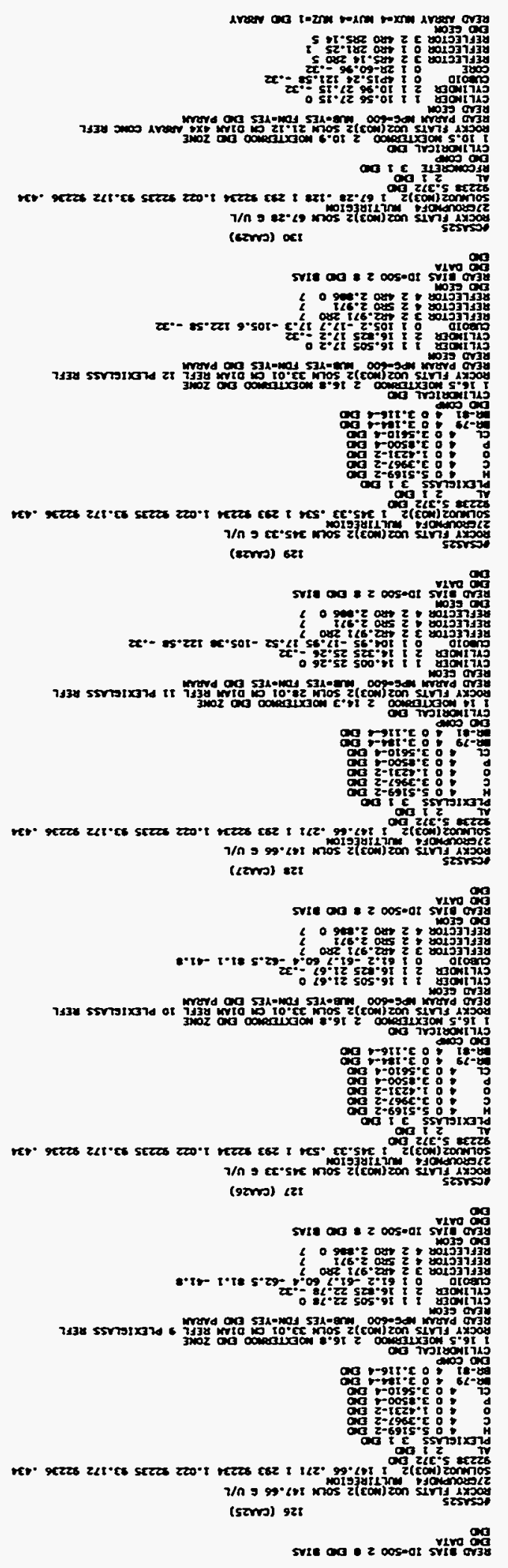

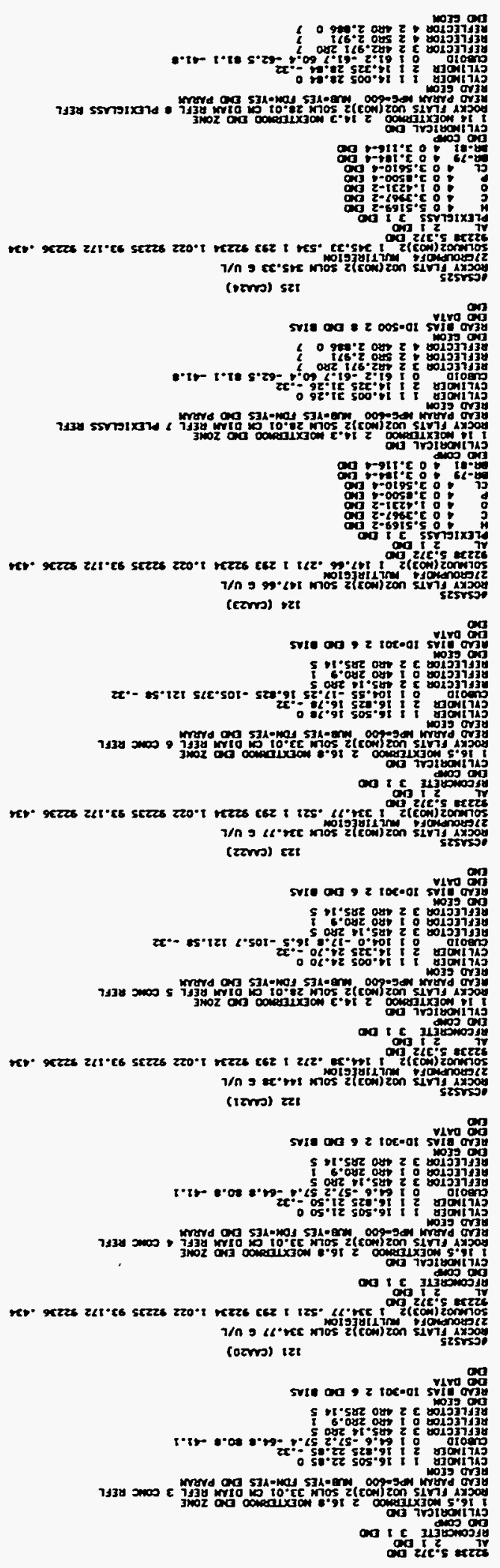




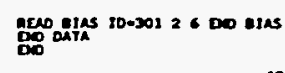

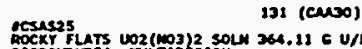

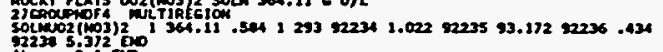

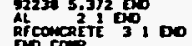

Critholicat go

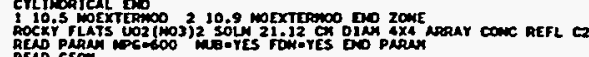

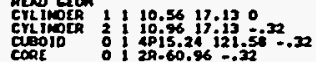

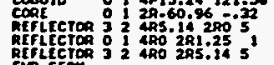

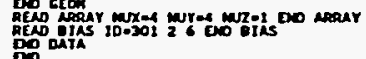

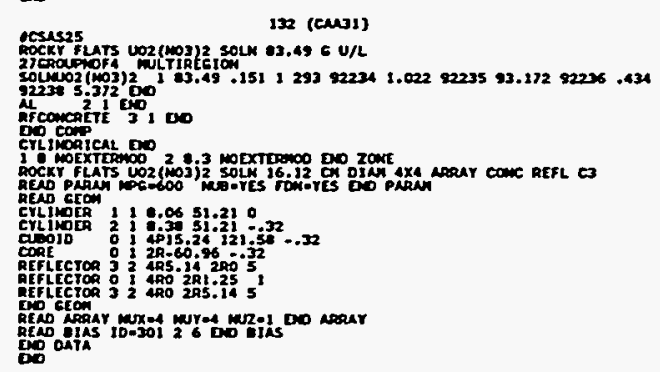

.csuses 233 (cons)

Gescses

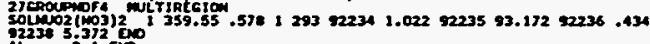

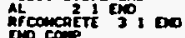

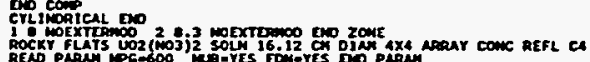

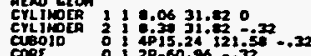

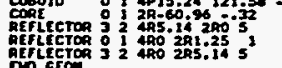

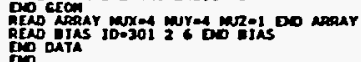

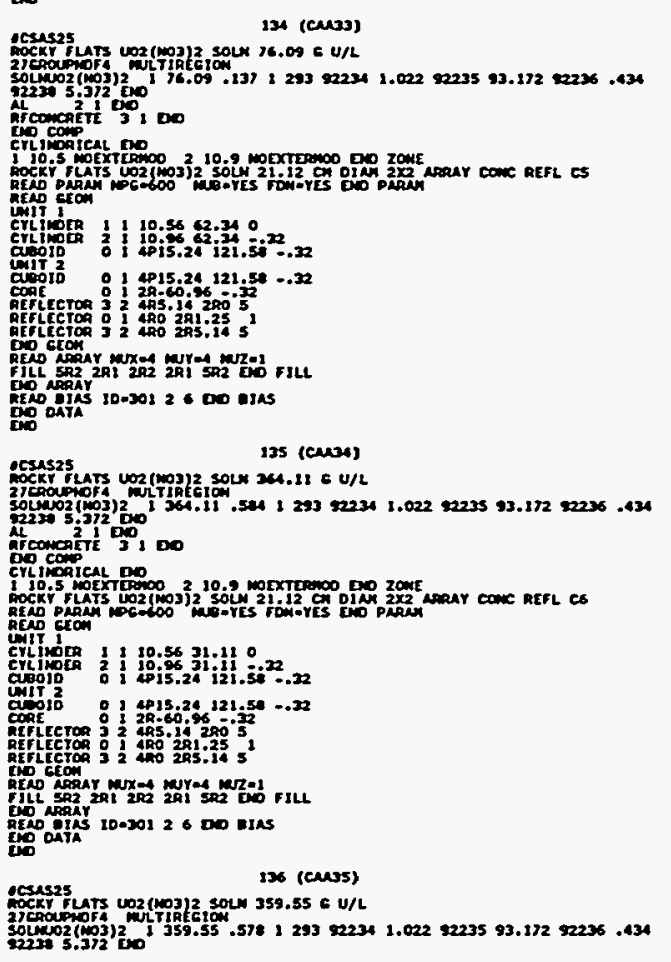

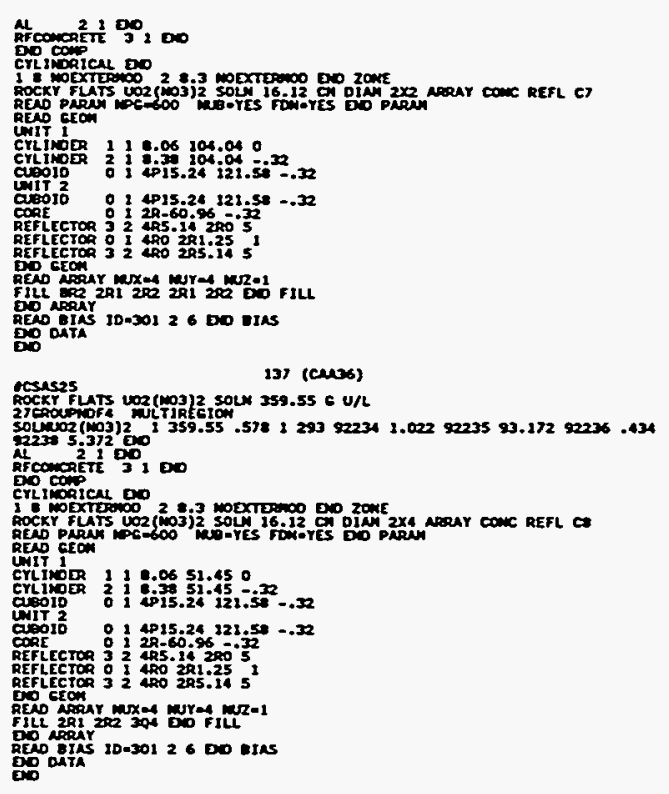

cesesss 139 (c)u3r)

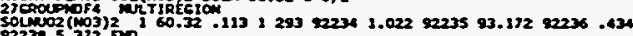
$25305,332.000$

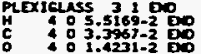

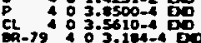

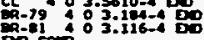

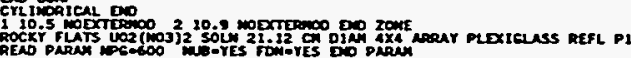
ateo pasen

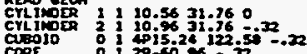

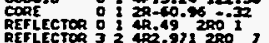

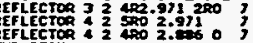

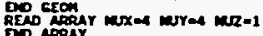

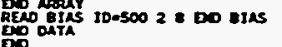

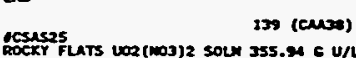

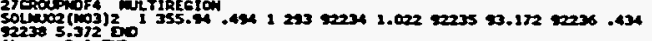

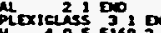

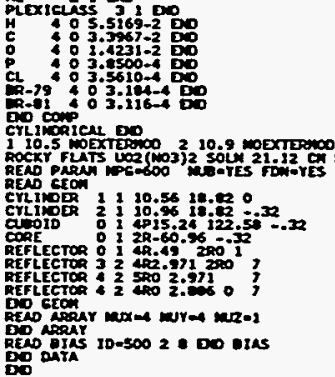

140 (curs)

gcters

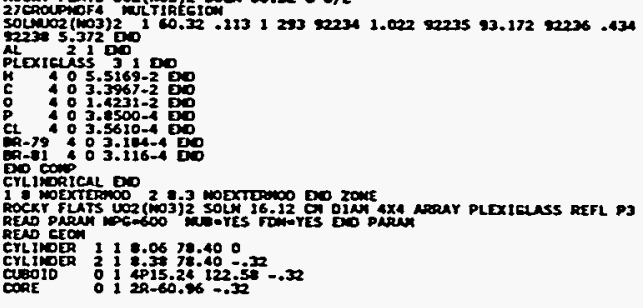



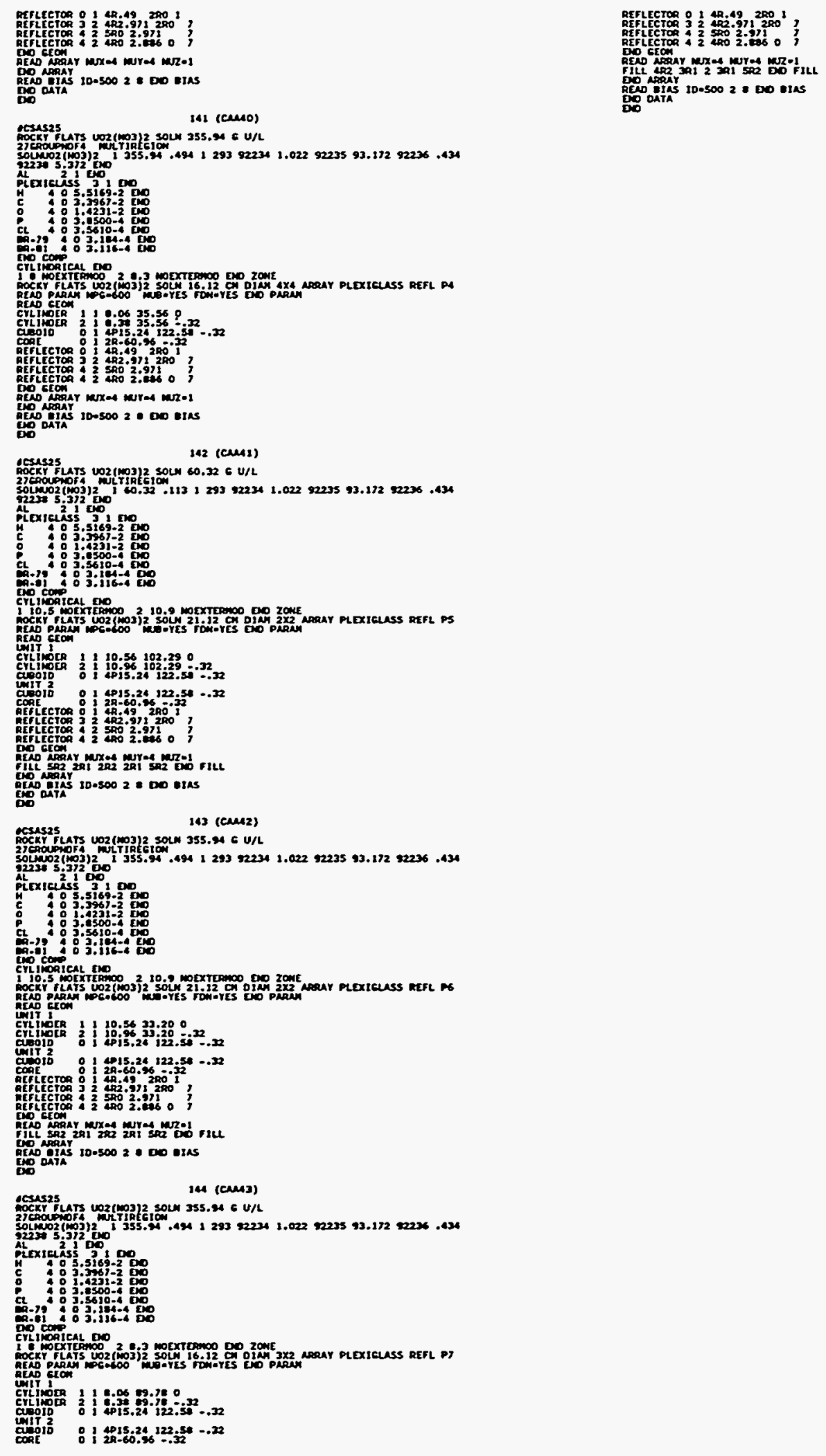


\section{APPENDIX E5: TABLE 5 INPUTS}

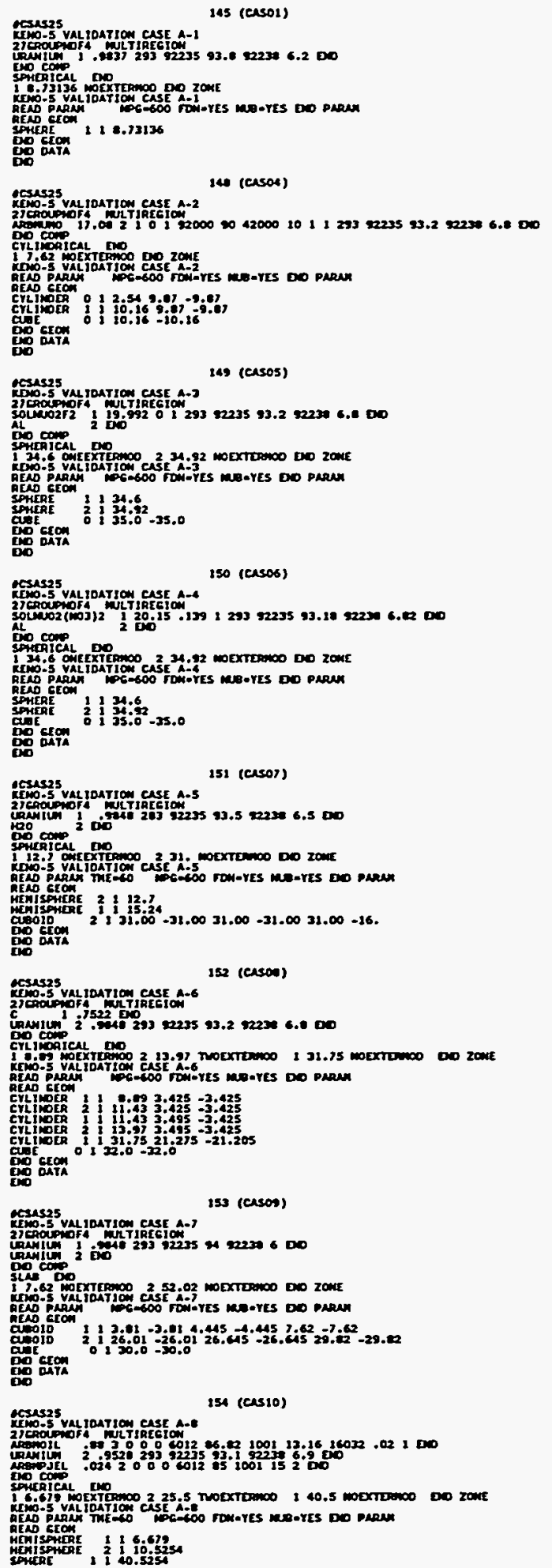




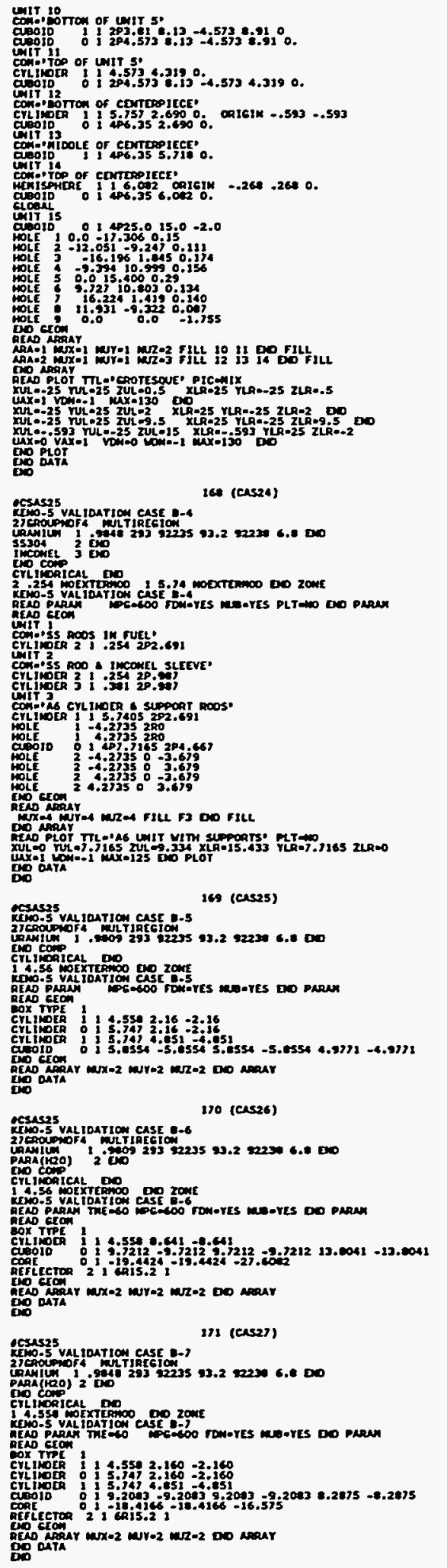

172 (casze)

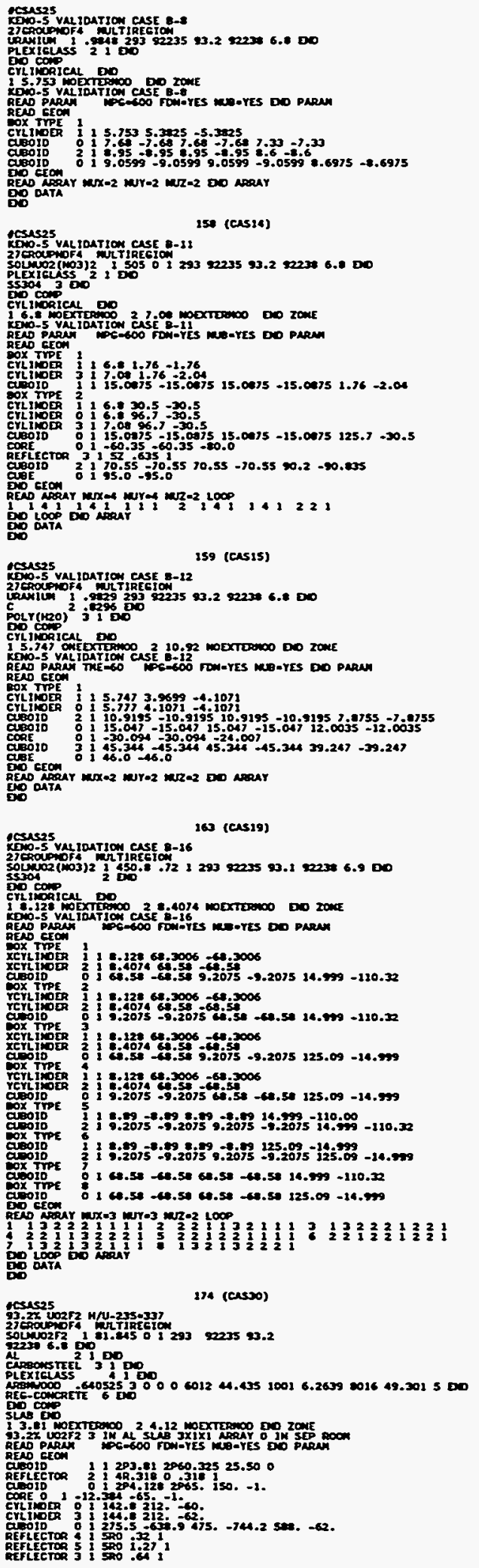



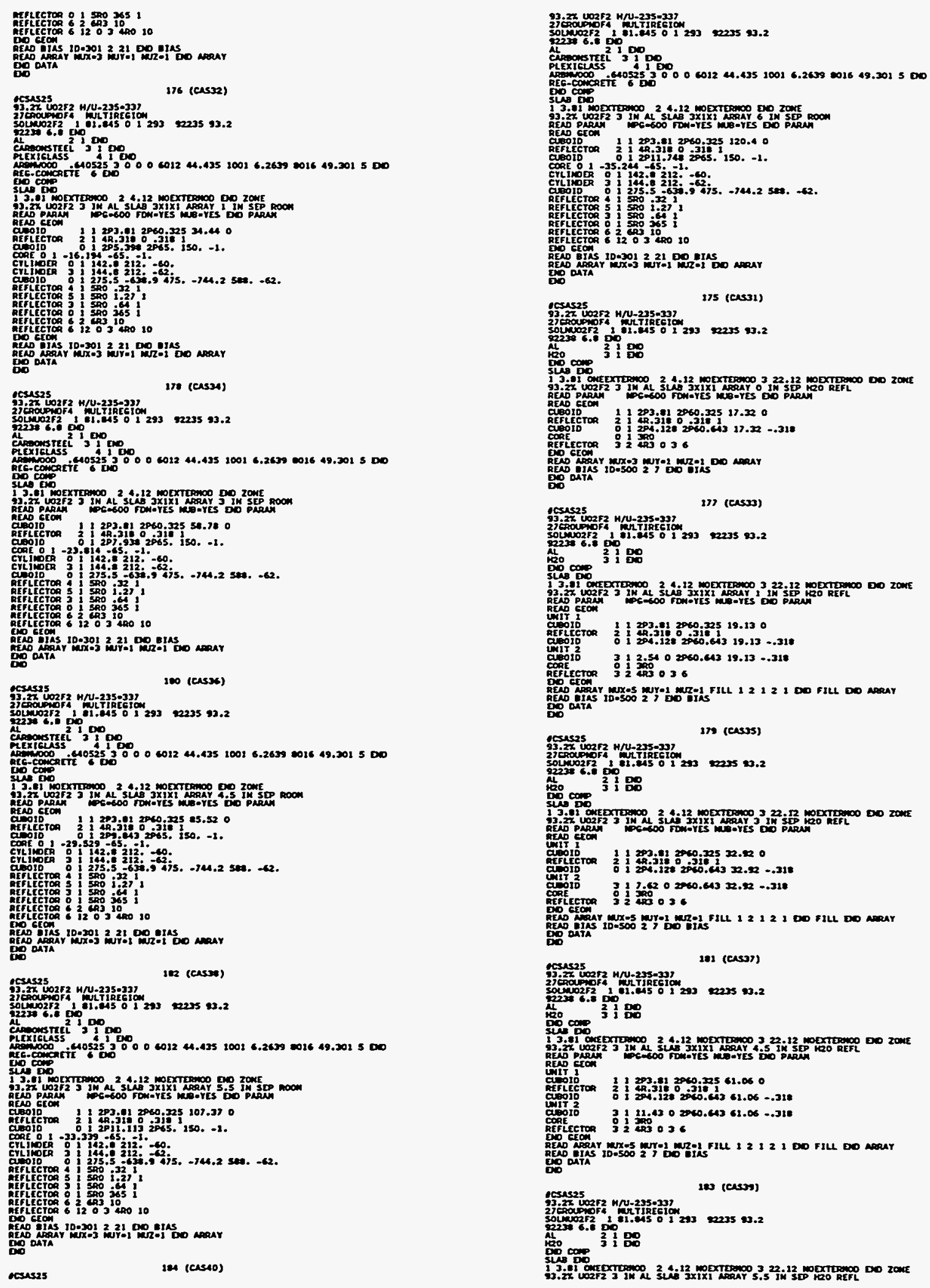


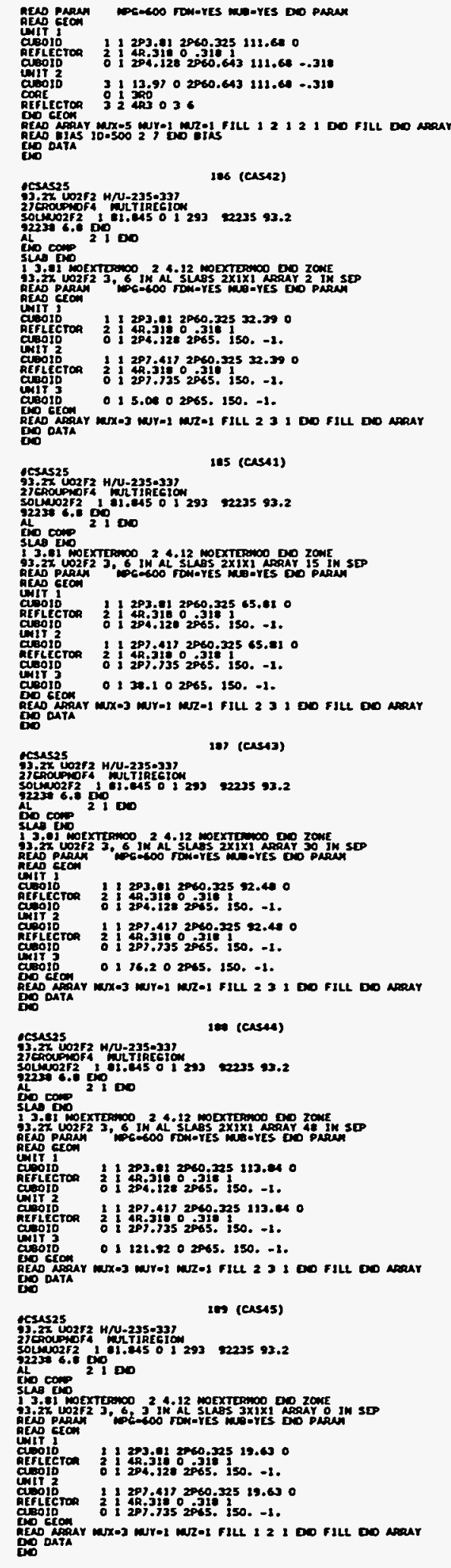

120 (ceses)

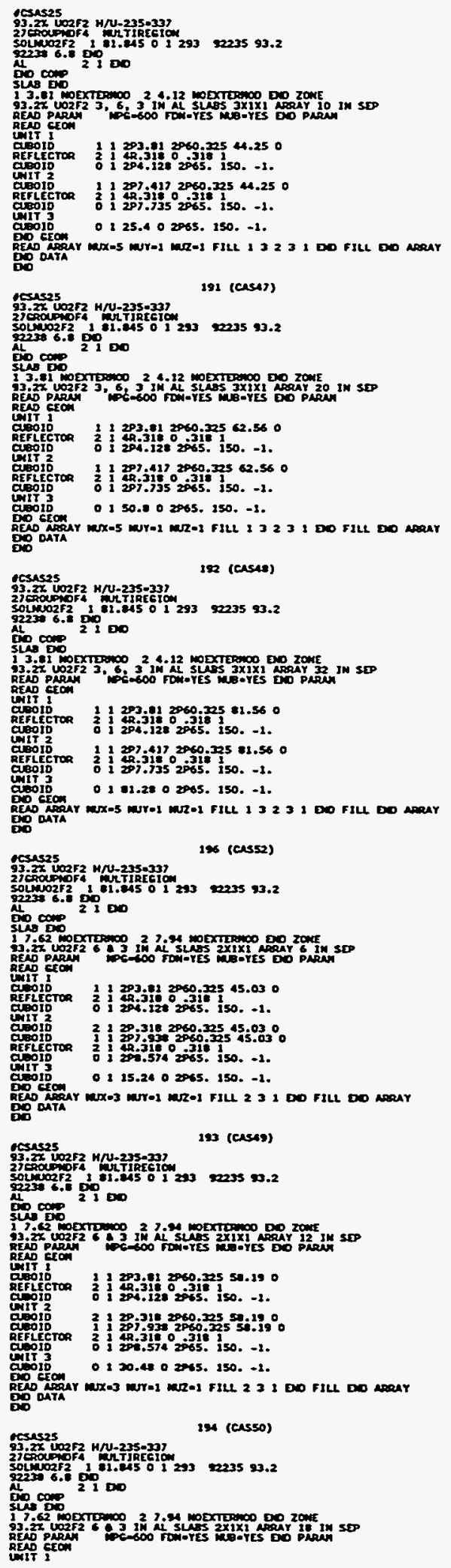




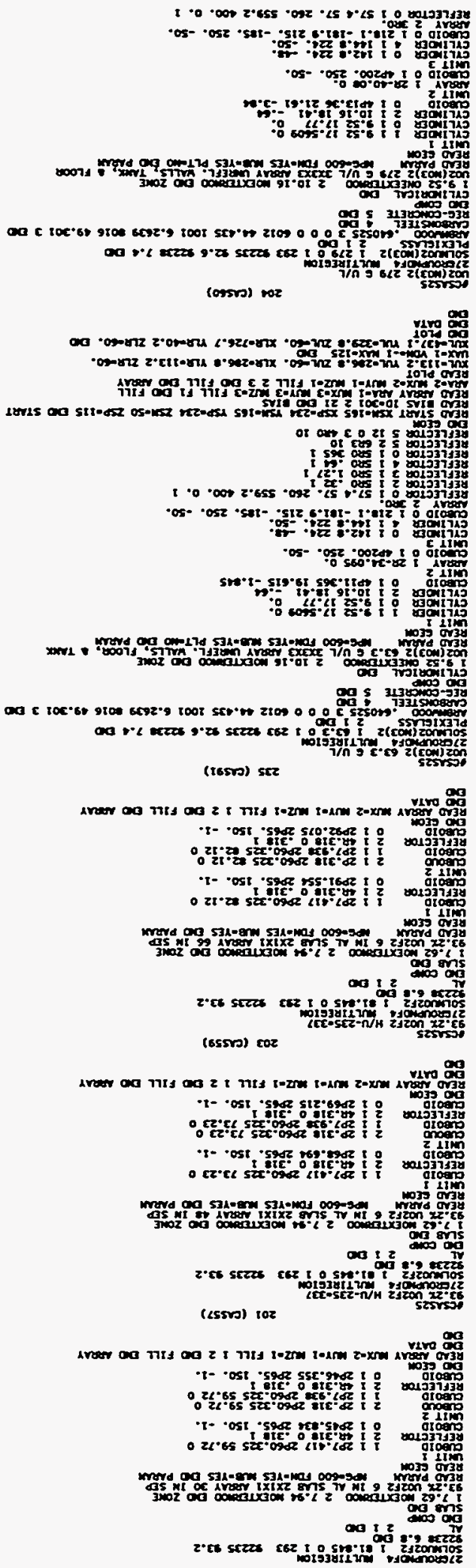

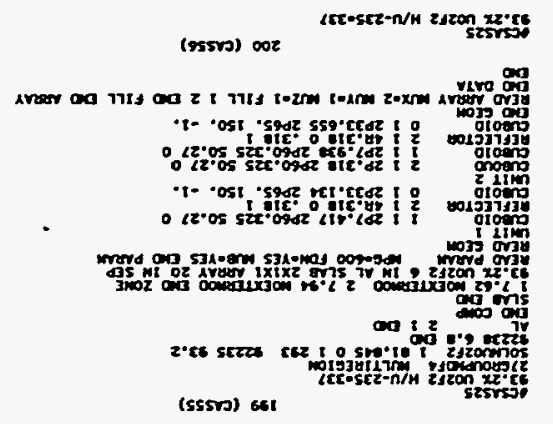

A

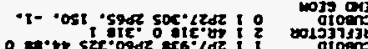
O.

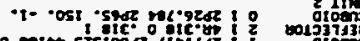

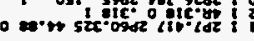

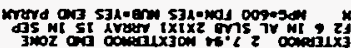

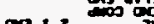

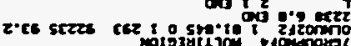

(tss) $46 \mathrm{~s}$

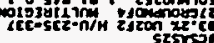

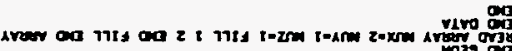

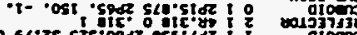

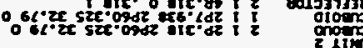

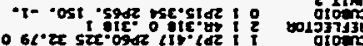

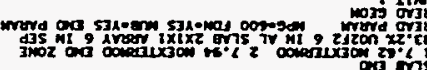

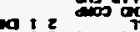

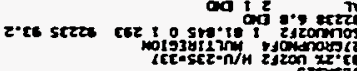

(ess) 202

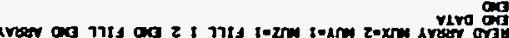

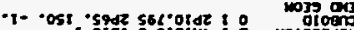

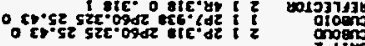

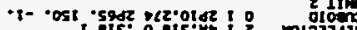

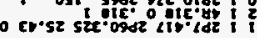

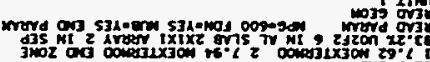

$0012=0000$

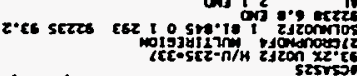

(rssos) *

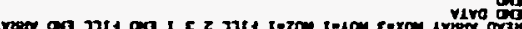

i- ont ishe o z.

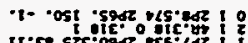

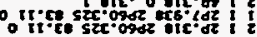

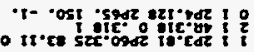

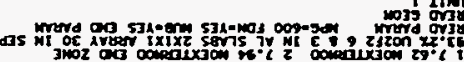

$\infty \mathrm{t} z \mathrm{C}_{\infty} \mathrm{O}$

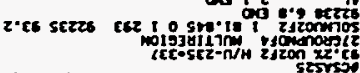

(1ssm) 201

4an

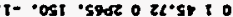

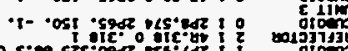

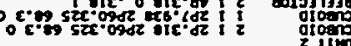

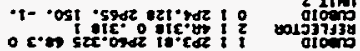




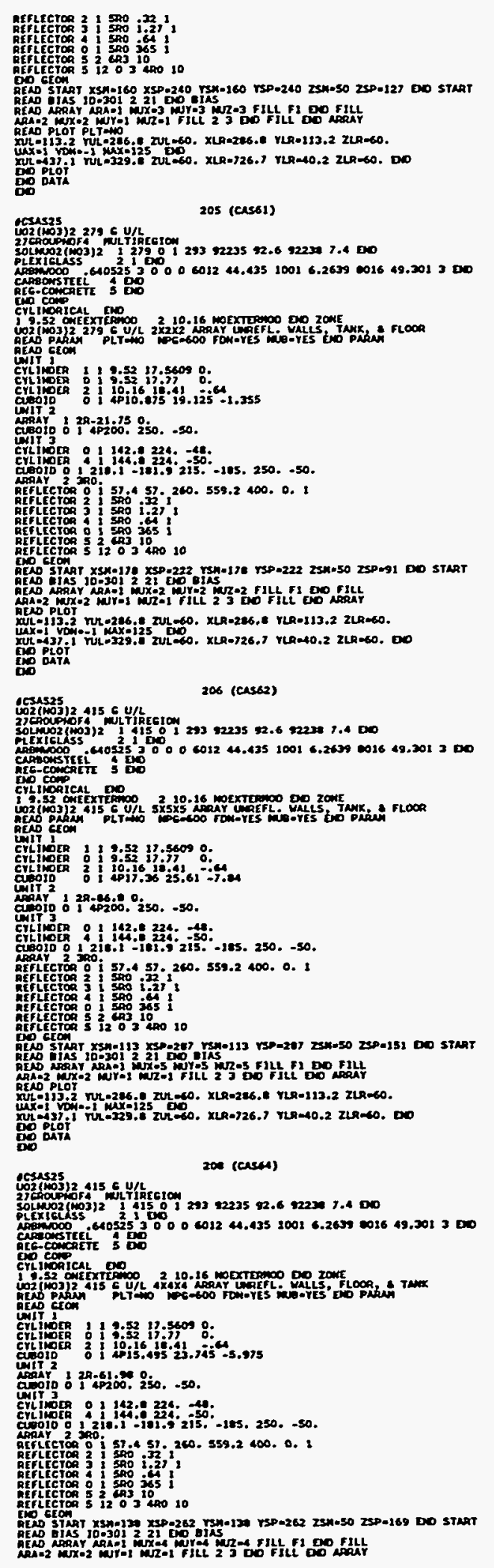

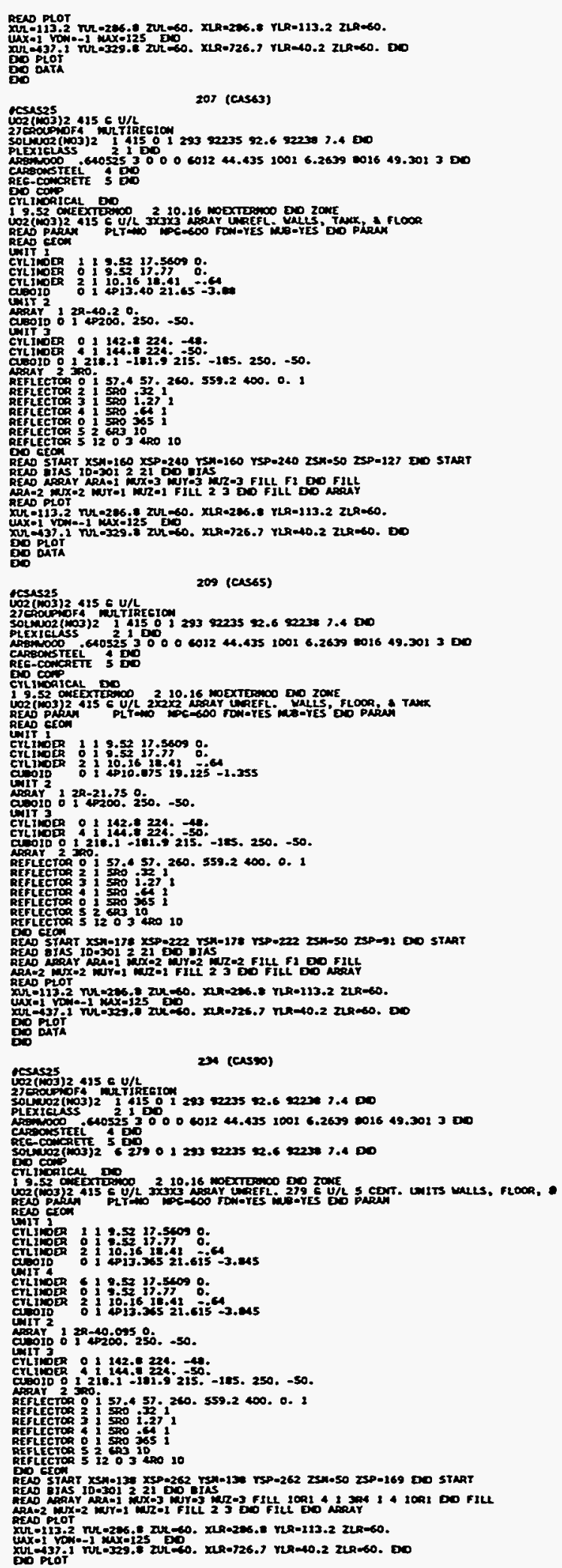



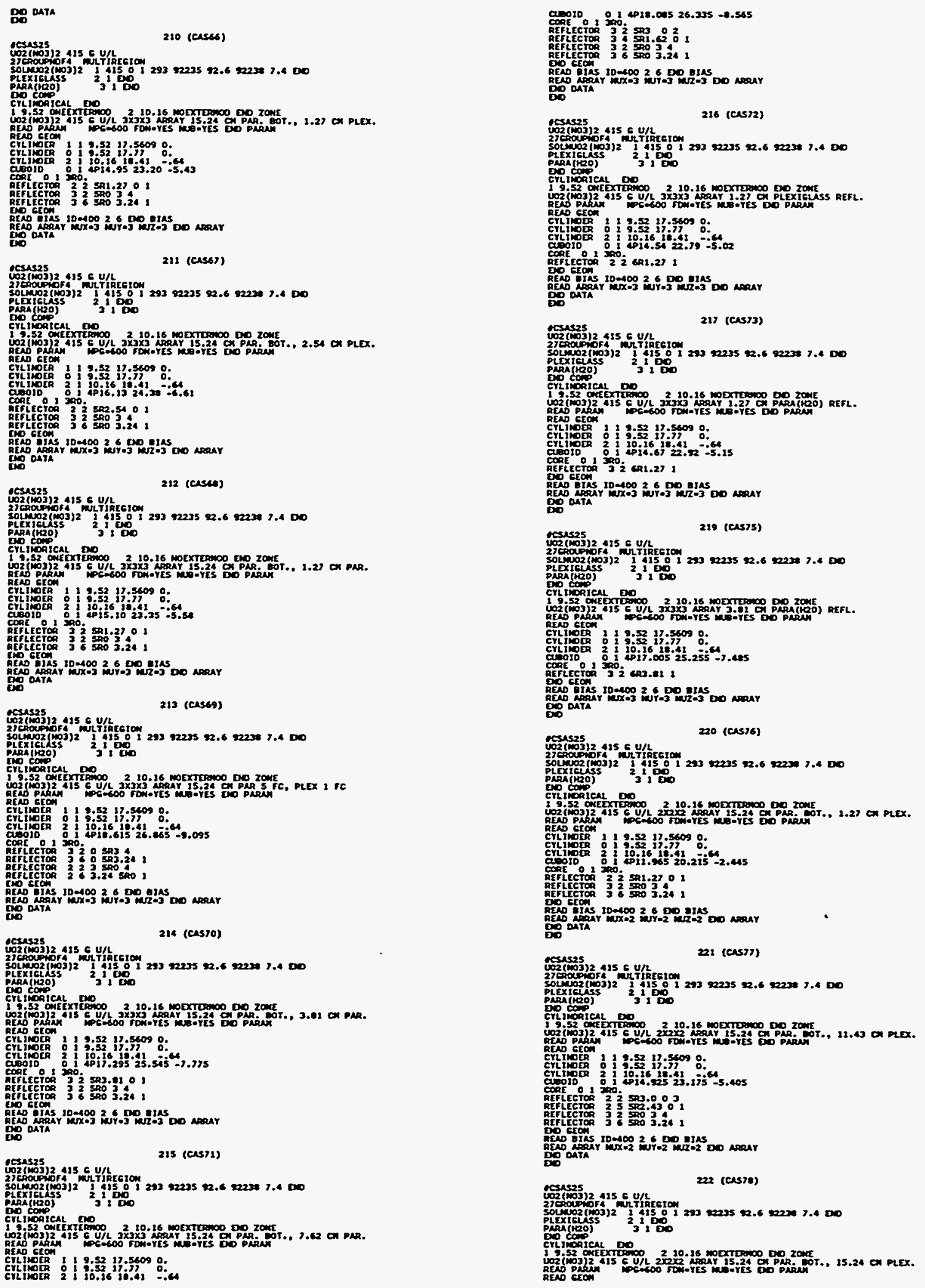

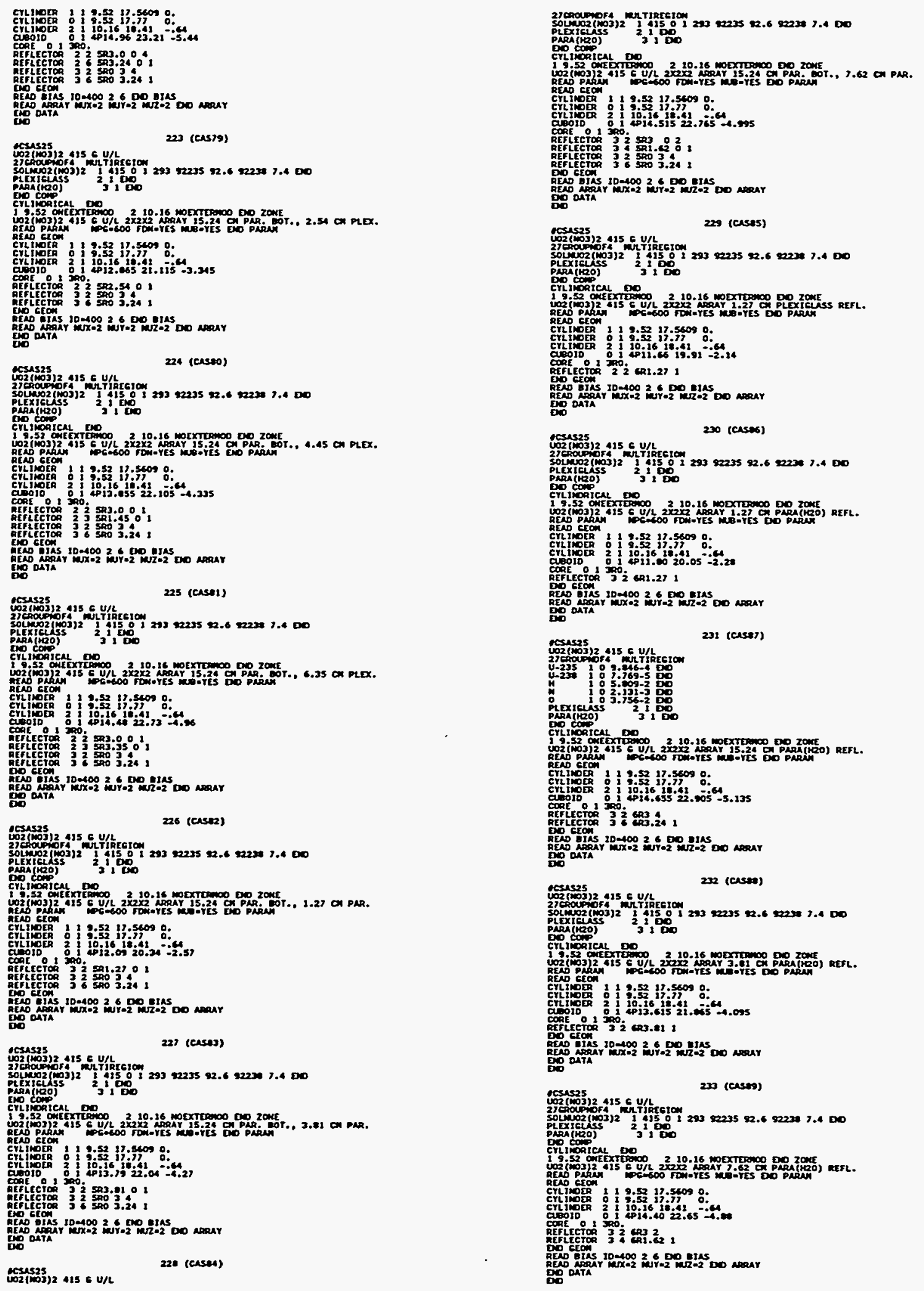


\section{APPENDIX E6: TABLE 6 INPUTS}
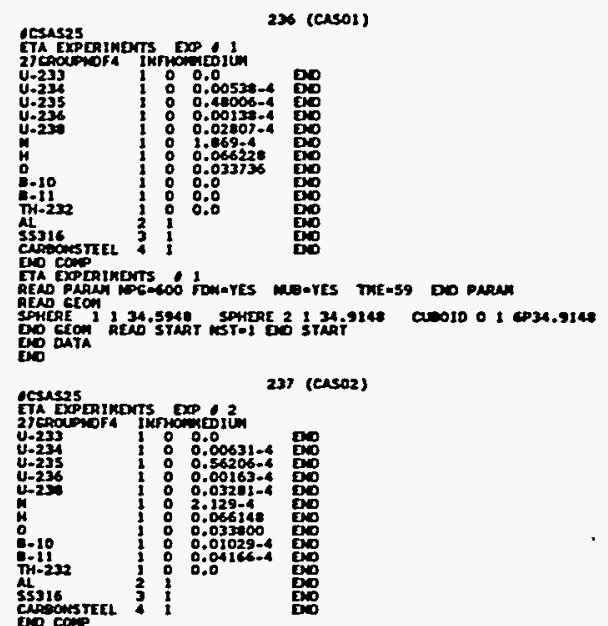

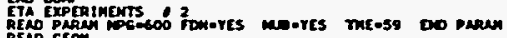

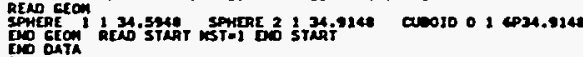
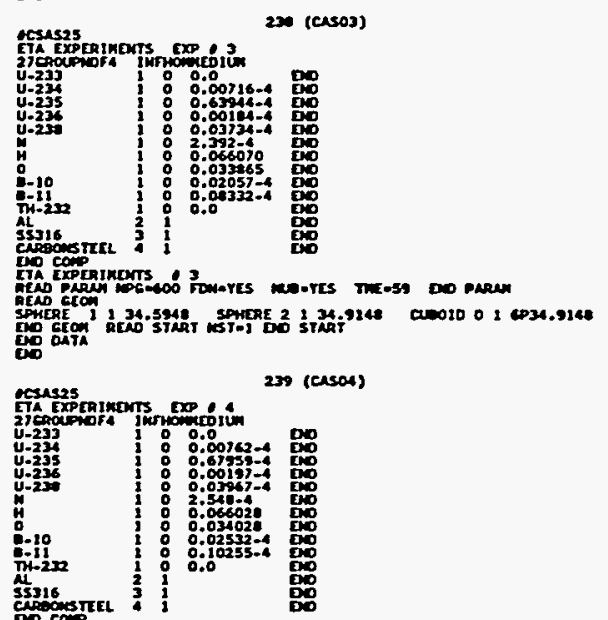

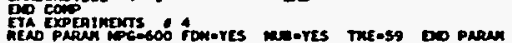

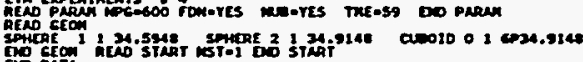
Do

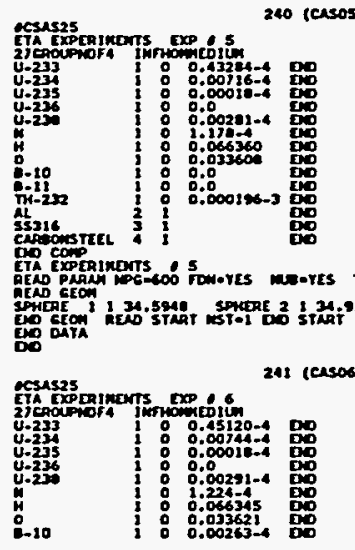

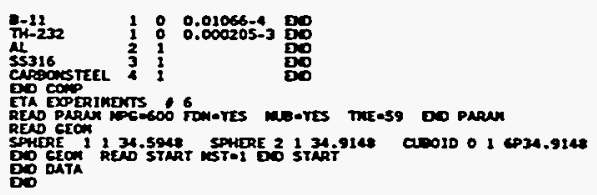
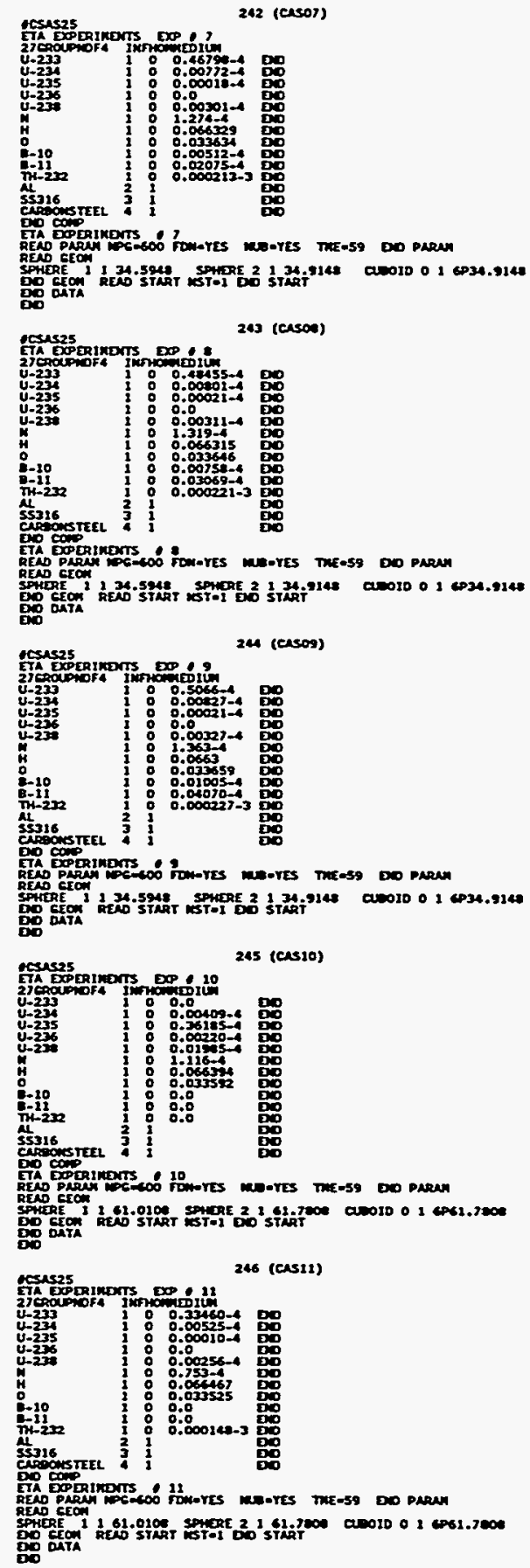

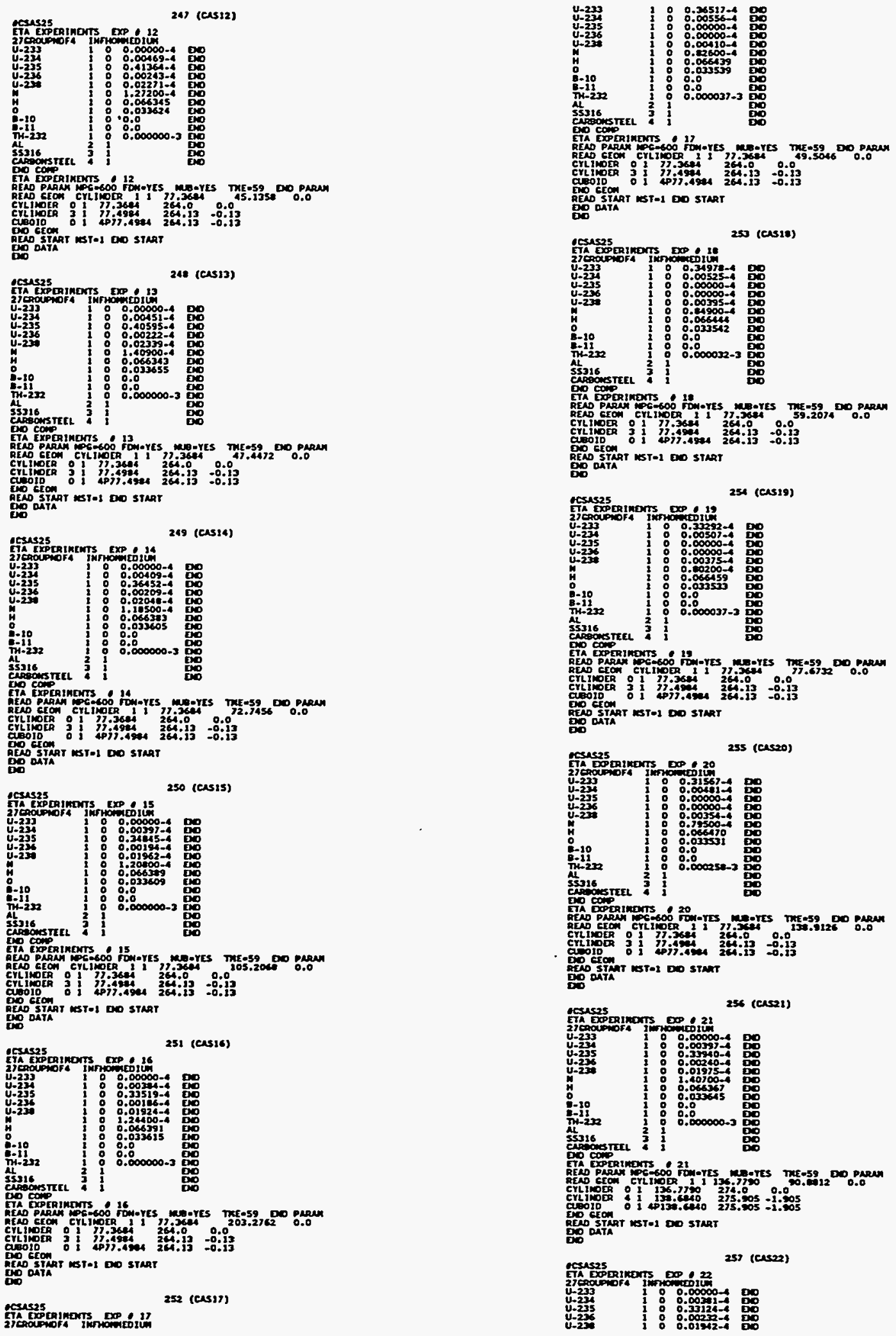


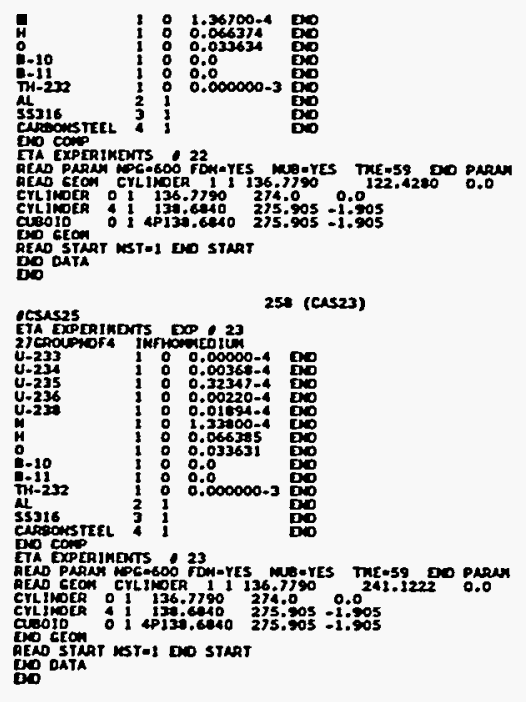

